\title{
Stereoselective Palladium-Catalyzed Arylation of Exo-Glycals with Aryl Iodides
}

\author{
Jeffery Regier, Supriya Ghanty and Yuri Bolshan*
}

Faculty of Science, Ontario Tech University, 2000 Simcoe Street North, Oshawa, Ontario L1H 7K4, Canada

*Email: yuri.bolshan@ontariotechu.ca

\section{Supporting Information}




\section{Contents}

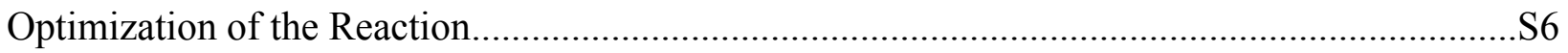

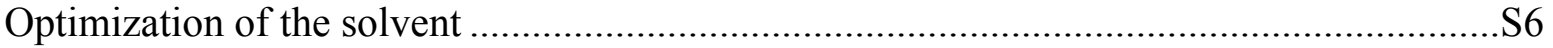

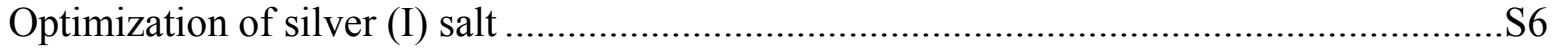

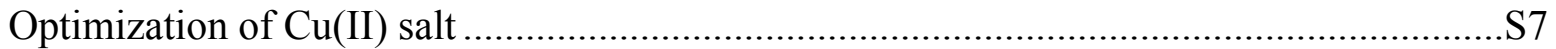

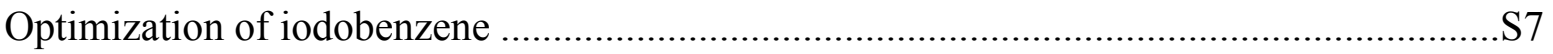

Optimization of temperature and palladium catalyst...........................................................

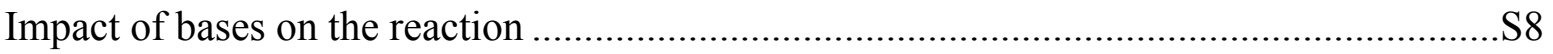

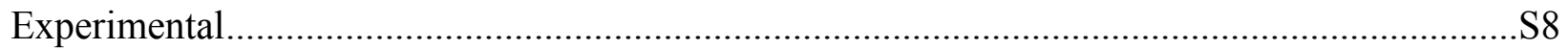

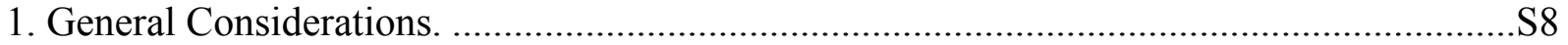

2. Experimental Conditions for Starting Exo-glycals and Precursors. ......................................S9

2.1. General Procedure for the Bromine Oxidation of Pentoses. .........................................S9

2.2. General Procedure for the Silylation of Carbohydrate Lactones....................................S9

(1') 2,3,4,6-Tetrakis-O-(triethylsilyl)-D-glucopyrano-1,5-lactone. ……………............S10

(2') 2,3,5-Tris-O-(triethylsilyl)-D-ribofurano-1,4-lactone. .............................................S10

(3') 2,3,5-Tris-O-(triethylsilyl)-D-xylonofurano-1,4-lactone.........................................S10

(4') 2,3,5-Tris-O-(triethylsilyl)-D-arabinofurano-1,4-lactone........................................S11

2.3. General Procedure for the Oxidation of Benzyl-Protected Carbohydrates. .................S1 1

(5') 2,3,4,6-Tetra-0-benzyl-D-glucono-1,5-lactone. ………………….........................S1

(6') 2,3,4,6-Tetra-O-benzyl-D-galacto-1,5-lactone ……..............................................S12

(7') 2,3,4,6-Tetra-O-benzyl-D-manno-1,5-lactone......................................................S12

(8') 2,3,5-tri-0-benzyl-D-arabino-1,4-lactone. ……………………….......................

2.4. General Procedure for the Methylenation of Sugar Lactones using Tebbe's reagent..S13

(1) 3,4,5,7-Tetrakis-O-(triethylsilyl)-2,6-anhydro-1-deoxy-D-glucono-hept-1-enitol....S14

(2) 3,4,6-Tris-O-(triethylsilyl)-2,5-anhydro-1-deoxy-D-ribino-hex-1-enitol..................S14

(3) 3,4,6-Tris-O-(triethylsilyl)-2,5-anhydro-1-deoxy-D-xylono-hex-1-enitol. ...............S14

(4) 3,4,6-Tris-O-(triethylsilyl)-2,5-anhydro-1-deoxy-D-arabino-hex-1-enitol. ..............S14

(5) 3,4,5,7-Tetra-O-benzyl-2,6-anhydro-1-deoxy-D-gluco-hept-1-enitol......................S15

(6) 3,4,5,7-Tetra-O-benzyl-2,6-anhydro-1-deoxy-D-galacto-hept-1-enitol....................S15

(7) 3,4,5,7-Tetra-O-benzyl-2,6-anhydro-1-deoxy-D-manno-hept-1-enitol.....................S15

(8) 3,4,6-Tri-O-benzyl-2,5-anhydro-1-deoxy-D-arabino-hex-1-enitol. ...........................S16 
2.5 Synthesis of 3,4,5,7-Tetra-O-benzyl-2,6-anhydro-1-deoxy-D-gluco-hept-1-enitol. (9)

3. Experimental Conditions for Aryl-Exo-Glucals.........................................................S17

3.1. General Procedure for the Arylation of Exo-Glycals. ............................................ 17

(1a) 3,4,5,7-Tetra-O-triethylsilyl-2,6-anhydro-1-1-deoxy-D-gluco-hept-1-enitol.........S17

(1b) 3,4,5,7-Tetra-O-triethylsilyl-2,6-anhydro-1-(3-methylphenyl)-1-deoxy-D-glucohept-1-enitol.

(1c) 3,4,5,7-Tetra-O-triethylsilyl-2,6-anhydro-1-(3-methoxylphenyl)-1-deoxy-D-glucohept-1-enitol. S18

(1d) 3,4,5,7-Tetra-O-triethylsilyl-2,6-anhydro-1-(1-naphthyl)-1-deoxy-D-gluco-hept-1enitol. S18

(1e) 3,4,5,7-Tetra-O-triethylsilyl-2,6-anhydro-1-(4-biphenyl)-1-deoxy-D-gluco-hept-1enitol. S19

(1f) 3,4,5,7-Tetra-O-triethylsilyl-2,6-anhydro-1-(4-fluorophenyl)-1-deoxy-D-gluco-hept1-enitol. S19

(1g) 3,4,5,7-Tetra-O-triethylsilyl-2,6-anhydro-1-(4-chlorophenyl)-1-deoxy-D-gluco-hept1-enitol. S19

(1h) 3,4,5,7-Tetra-O-triethylsilyl-2,6-anhydro-1-(4-bromophenyl)-1-deoxy-D-gluco-hept1-enitol. S20

(1i) 3,4,5,7-Tetra-O-triethylsilyl-2,6-anhydro-1-(3-bromophenyl)-1-deoxy-D-gluco-hept1-enitol. S20

(1j) 3,4,5,7-Tetra-O-triethylsilyl-2,6-anhydro-1-(3-nitrophenyl)-1-deoxy-D-gluco-hept-1enitol. S21

(1k) 3,4,5,7-Tetra-O-triethylsilyl-2,6-anhydro-1-(4-cyanophenyl)-1-deoxy-D-gluco-hept1-enitol. S2 1

(11) 3,4,5,7-Tetra-O-triethylsilyl-2,6-anhydro-1-(3-cyanophenyl)-1-deoxy-D-gluco-hept1-enitol. .S21

(1m) 3,4,5,7-Tetra-O-triethylsilyl-2,6-anhydro-1-(4-trifluorophenyl)-1-deoxy-D-glucohept-1-enitol. S22

(1n) 3,4,5,7-Tetra-O-triethylsilyl-2,6-anhydro-1-(2-chloro-5-trifluorophenyl)-1-deoxy-Dgluco-hept-1-enitol. S22

(10) 3,4,5,7-Tetra-O-triethylsilyl-2,6-anhydro-1-(3-trifluorophenyl)-1-deoxy-D-glucohept-1-enitol. S23

(1p) 3,4,5,7-Tetra-O-triethylsilyl-2,6-anhydro-1-(4-(hydroxymethyl)-phenyl)-1-deoxy-Dgluco-hept-1-enitol. S23 
(1q) 3,4,5,7-Tetra-O-triethylsilyl-2,6-anhydro-1-(3-(hydroxymethyl)-phenyl)-1-deoxy-Dgluco-hept-1-enitol.

(1r) 3,4,5,7-Tetra-O-triethylsilyl-2,6-anhydro-1-(3-(hydroxy(phenyl)methyl)phenyl)-1deoxy-D-gluco-hept-1-enitol. S24

(1s) 3,4,5,7-Tetra-O-triethylsilyl-2,6-anhydro-1-(3-formylphenyl)-1-deoxy-D-gluco-hept1-enitol. .S25

(1t) 3,4,5,7-Tetra-O-triethylsilyl-2,6-anhydro-1-(4-formylphenyl)-1-deoxy-D-gluco-hept1-enitol. S25

(1u) 3,4,5,7-Tetra-O-triethylsilyl-2,6-anhydro-1-(4-carboxyphenyl)-1-deoxy-D-glucohept-1-enitol. S25

(1v) 3,4,5,7-Tetra-O-triethylsilyl-2,6-anhydro-1-(3-(methoxycarbonyl)phenyl)-1-deoxyD-gluco-hept-1-enitol. S26

(1w) 3,4,5,7-Tetra-O-triethylsilyl-2,6-anhydro-1-(4-(Methoxycarbonyl)phenyl)-1-deoxyD-gluco-hept-1-enitol. S26

(2a) 3,4,6-Tri-O-triethylsilyl-2,5-anhydro-1-phenyl-1-deoxy-D-ribino-hex-1-enitol. ...S27 (2b) 3,4,6-Tri-O-triethylsilyl-2,5-anhydro-1-(3-nitrophenyl)-1-deoxy-D-ribino-hex-1enitol....... S27

(2c) 3,4,6-Tri-O-triethylsilyl-2,5-anhydro-1-(4-cyanophenyl)-1-deoxy-D-ribino-hex-1enitol. S27

(2d) 3,4,6-Tri-O-triethylsilyl-2,5-anhydro-1-(4-(hydroxymethyl)phenyl)-1-deoxy-Dribino-hex-1-enitol. S28

(2e) 3,4,6-Tri-O-triethylsilyl-2,5-anhydro-1-(4-formylphenyl)-1-deoxy-D-ribino-hex-1enitol. S28

(2f) 3,4,6-Tri-O-triethylsilyl-2,5-anhydro-1-((4-methoxycarbonyl)phenyl)-1-deoxy-Dribino-hex-1-enitol.

(2g) 3,4,6-Tri-O-triethylsilyl-2,5-anhydro-1-(4-chlorophenyl)-1-deoxy-D-ribino-hex-1enitol. S29

(2h) 3,4,6-Tri-O-triethylsilyl-2,5-anhydro-1-(4.bromophenyl)-1-deoxy-D-ribino-hex-1enitol.

(3a) 3,4,6-Tri-O-triethylsilyl-2,5-anhydro-1-phenyl-1-deoxy-D-xylono-hex-1-enitol...S30 (3b) 3,4,6-Tri-O-triethylsilyl-2,5-anhydro-1-(3-nitrophenyl)-1-deoxy-D-xylono-hex-1enitol. S30

(3c) 3,4,6-Tri-O-triethylsilyl-2,5-anhydro-1-(4-cyanophenyl)-1-deoxy-D-xylono-hex-1enitol. S30 
(3d) 3,4,6-Tri-O-triethylsilyl-2,5-anhydro-1-(4-(hydroxymethyl)-phenyl)-1-deoxy-Dxylono-hex-1-enitol.

S31

(3e) 3,4,6-Tri-O-triethylsilyl-2,5-anhydro-1-(4-formylphenyl)-1-deoxy-D-xylono-hex-1enitol.

(3f) 3,4,6-Tri-O-triethylsilyl-2,5-anhydro-1-(4-(methoxycarbonyl)phenyl)-1-deoxy-Dxylono-hex-1-enitol. $\mathrm{S} 32$

(4a) 3,4,6-Tri-O-triethylsilyl-2,5-anhydro-1-phenyl-1-deoxy-D-arabino-hex-1-enitol. .S32

(4b) 3,4,6-Tri-O-triethylsilyl-2,5-anhydro-1-(3-nitrophenyl)-1-deoxy-D-arabino-hex-1enitol. S32

(4c) 3,4,6-Tri-O-triethylsilyl-2,5-anhydro-1-(4-cyanophenyl)-1-deoxy-D-arabino-hex-1enitol. $\mathrm{S} 33$

(4d) 3,4,6-Tri-O-triethylsilyl-2,5-anhydro-1-(4-(hydroxymethyl)-phenyl)-1-deoxy-Darabino-hex-1-enitol. S33

(4e) 3,4,6-Tri-O-triethylsilyl-2,5-anhydro-1-(4-formylphenyl)-1-deoxy-D-arabino-hex-1enitol. S34

(4f) 3,4,6-Tri-O-triethylsilyl-2,5-anhydro-1-(4-(methoxycarbonyl)phenyl)-1-deoxy-Darabino-hex-1-enitol. S34

(5a) 3,4,5,7-Tetra-O-benzyl-2,6-anhydro-1-phenyl-1-deoxy-D-gluco-hept-1-enitol. ....S34 (6a) 3,4,5,7-Tetra-O-benzyl-2,6-anhydro-1-phenyl-1-deoxy-D-galacto-hept-1-enitol...S35 (7a) 3,4,5,7-Tetra-O-benzyl-2,6-anhydro-1-phenyl-1-deoxy-D-manno-hept-1-enitol. ..S35 (8a) 3,4,6-Tri-O-benzyl-2,5-anhydro-1-phenyl-1-deoxy-D-arabino-hex-1-enitol..........S36 (9a) 3,4,5,7-Tetra-O-acetyl-2,6-anhydro-1-phenyl-1-deoxy-D-glucono-hept-1-enitol. .S36 (1x) 3,4,5,7-Tetra-O-triethylsilyl-2,6-anhydro-1-deoxy-1-phenyl-D-glycero-L-guloheptitol S37

3.2. Synthesis of 1-chloro-2-(4-ethoxybenzyl)-4-iodobenzene (10). S37

(11) 3,4,5,7-Tetra-O-benzyl-2,6-anhydro-1-(4-chloro-3-(4-ethoxybenzyl)phenyl)-1deoxy-D-gluco-hept-1-enitol. S38

(12) 2,6-anhydro-1-deoxy-1-(4-chloro-3-(4-ethoxybenzyl)phenyl)-D-glycero-L-guloheptitol..... S39

4. ${ }^{1} \mathrm{H}$ and ${ }^{13} \mathrm{C}\left\{{ }^{1} \mathrm{H}\right\}$ NMR Spectra. $\mathrm{S} 40$ 


\section{Optimization of the Reaction}

\section{Optimization of the solvent}

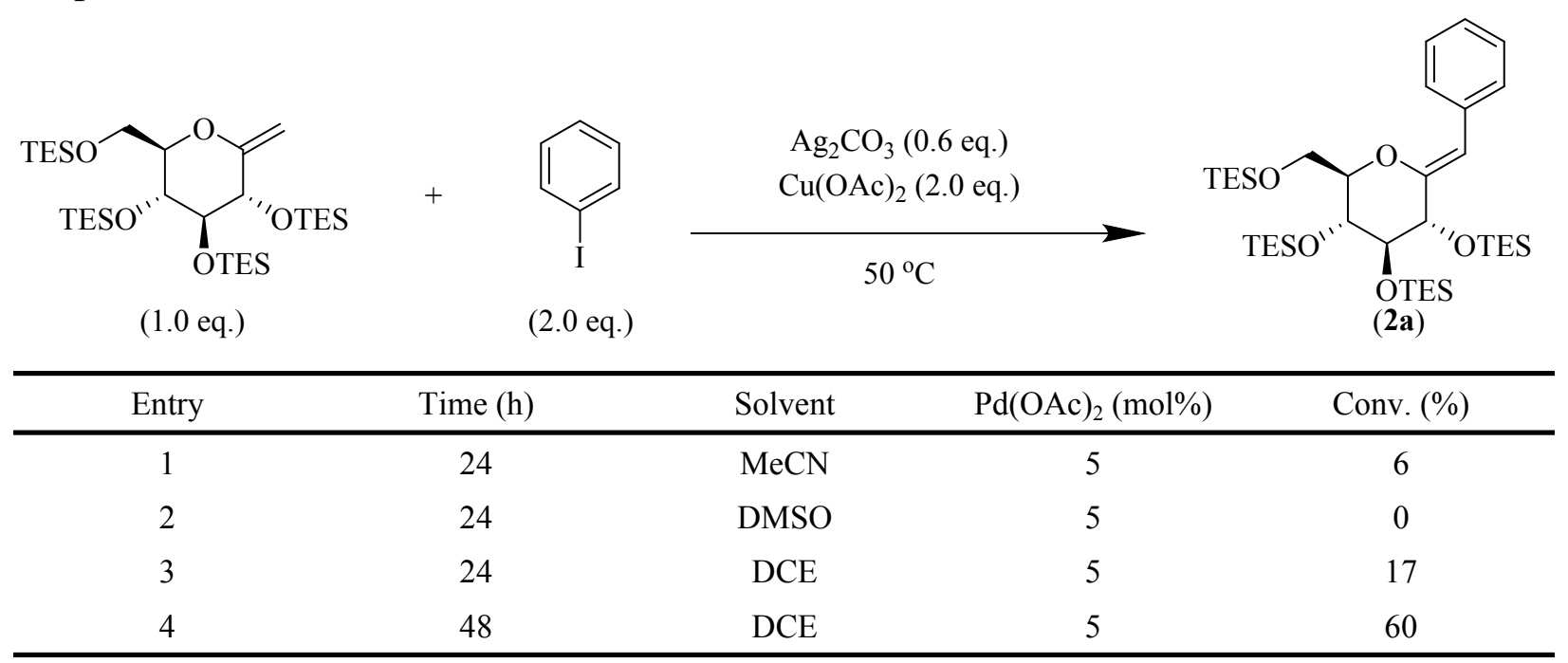

\section{Optimization of silver (I) salt}

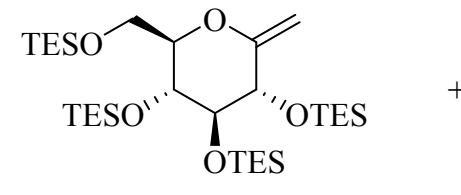

(1.0 eq.)

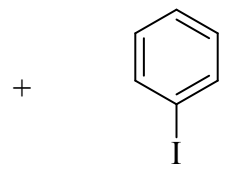

(2.0 eq.)

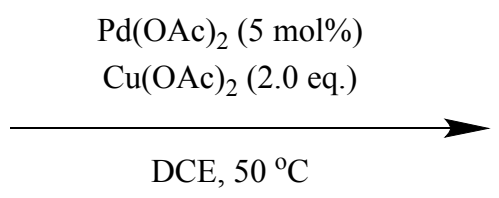

DCE, $50{ }^{\circ} \mathrm{C}$

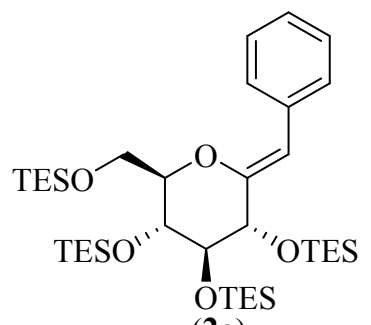

(2a)

\begin{tabular}{cccc}
\hline Entry & Time $(\mathrm{h})$ & $\mathrm{Ag}^{+}$Salt (eq.) & Conv. (\%) \\
\hline 1 & 48 & $\mathrm{Ag}_{2} \mathrm{CO}_{3}(0.6)$ & 60 \\
2 & 48 & $\operatorname{Ag}_{2} \mathrm{CO}_{3}(1.2)$ & 88 \\
3 & 48 & $\operatorname{AgNO}_{3}(1.2)$ & 0 \\
4 & 48 & $\operatorname{AgOAc}(1.2)$ & 96 \\
5 & 48 & $\operatorname{KOAc}(1.2)$ & 0 \\
6 & 24 & $\operatorname{AgOAc}(1.2)$ & 91 \\
7 & 24 & $\operatorname{AgOAc}(0.9)$ & 75 \\
\hline
\end{tabular}




\section{Optimization of $\mathrm{Cu}(\mathrm{II})$ salt}

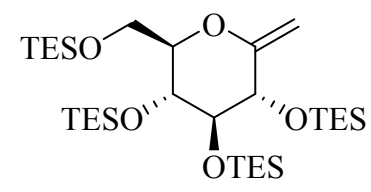

(1.0 eq.)

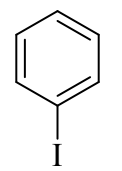

(2.0 eq.)

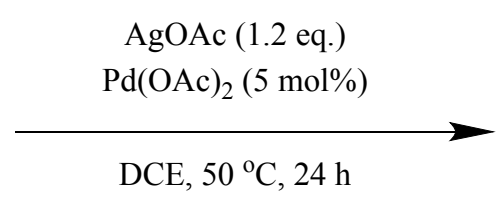

DCE, $50{ }^{\circ} \mathrm{C}, 24 \mathrm{~h}$

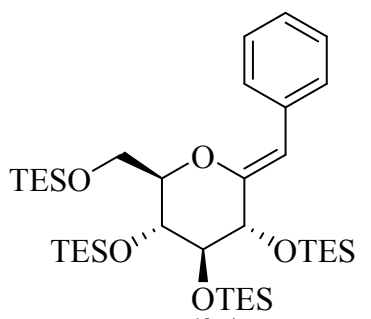

(2a)

\begin{tabular}{ccc}
\hline Entry & $\mathrm{Cu}^{2+}$ Salt (eq.) & Conv. (\%) \\
\hline 1 & $\mathrm{Cu}(\mathrm{OAc})_{2}(2.0)$ & 91 \\
2 & $\mathrm{CuSO}_{4}(2.0)$ & 86 \\
3 & $\mathrm{CuCl}_{2}(2.0)$ & 74 \\
4 & $\mathrm{Cu}(\mathrm{OAc})_{2}(1.5)$ & 87 \\
\hline
\end{tabular}

\section{Optimization of iodobenzene}

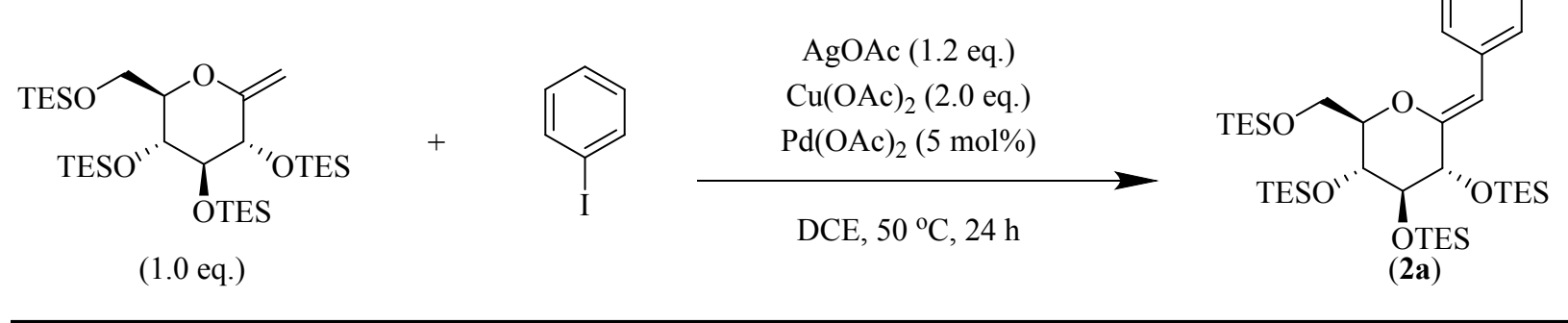

\begin{tabular}{ccc}
\hline Entry & Iodobenzene (eq.) & Conv. (\%) \\
\hline 1 & 2.0 & 91 \\
2 & 1.5 & 74 \\
3 & 1.1 & 63 \\
4 & 2.5 & 82 \\
\hline
\end{tabular}

\section{Optimization of temperature and palladium catalyst}

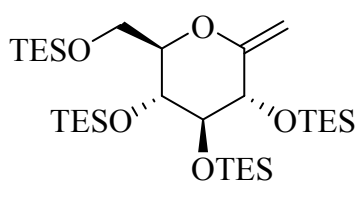

(1.0 eq.)<smiles>Ic1ccccc1</smiles>

(2.0 eq.)

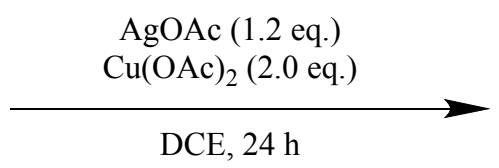

DCE, $24 \mathrm{~h}$

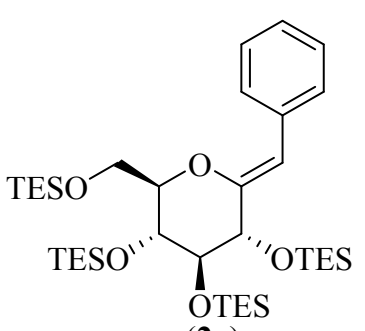




\begin{tabular}{cccc}
\hline Entry & Temperature $\left({ }^{\circ} \mathrm{C}\right)$ & $\operatorname{Pd}(\mathrm{OAc})_{2}(\mathrm{~mol} \%)$ & Conv. $(\%)$ \\
\hline 1 & 50 & $\operatorname{Pd}(\mathrm{OAc})_{2}(5)$ & 91 \\
2 & 60 & $\operatorname{Pd}(\mathrm{OAc})_{2}(5)$ & 97 \\
3 & 70 & $\operatorname{Pd}(\mathrm{OAc})_{2}(5)$ & 100 \\
4 & 70 & $\mathrm{Pd}(\mathrm{OAc})_{2}(3)$ & 92 \\
5 & 70 & $\mathrm{PdCl}_{2}(5)$ & 70 \\
6 & 70 & $\mathrm{PdCl}_{2}\left(\mathrm{PPh}_{3}\right)_{2}$ & 69 \\
7 & 70 & $\mathrm{Pd}\left(\mathrm{dba}_{2}\right.$ & 58 \\
8 & 70 & $\operatorname{Pd}\left(\mathrm{PPh}_{3}\right)_{4}$ & 0 \\
\hline
\end{tabular}

\section{Impact of bases on the reaction}

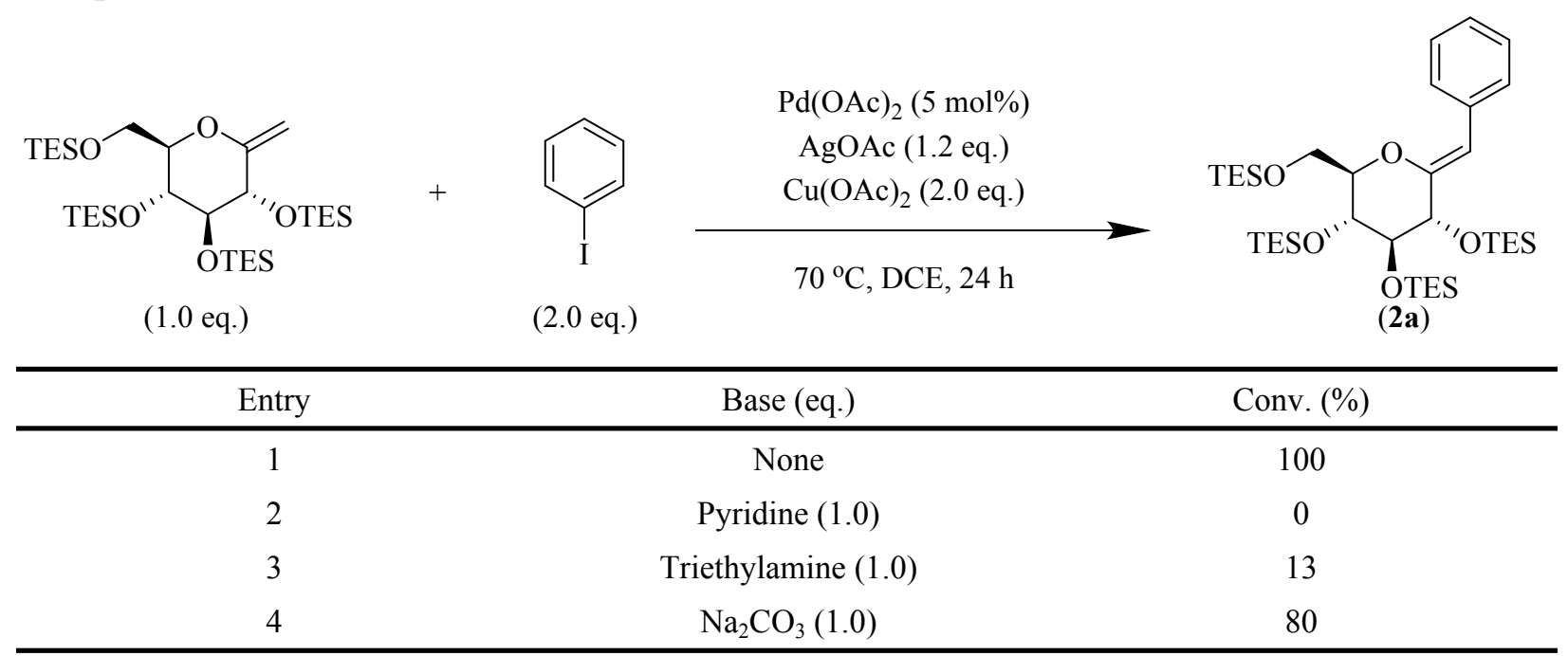

\section{Experimental}

\section{General Considerations.}

Reactions of exo-glycals with aryl iodides were performed in 2 dram vials with Drierite ${ }^{\mathrm{TM}}$ tubes fitted to the top of the vial. These reactions were heated in a reaction block on a hot plate. Reagents and materials were obtained from commercial sources without further purification unless otherwise stated. Reaction progress was monitored by TLC on glass-backed silica gel (60 $\AA, 250$ $\mu \mathrm{m}$ thickness, F254 indicator) with visualization under $254 \mathrm{~nm}$ ultraviolet light and staining by potassium permanganate $(\mathrm{KMnO} 4)$. NMR data was collected at $25^{\circ} \mathrm{C}$ using a Bruker Avance III $\mathrm{HD}$ spectrometer in deuterated solvents $\left(\mathrm{CDCl}_{3}\right.$ was obtained from Cambridge Isotope Laboratories, Inc., Andover, MA, USA). $1 \mathrm{H}$ and $13 \mathrm{C}[1 \mathrm{H}]$ were collected at 400 and $100 \mathrm{MHz}$, respectively, with chemical shifts expressed as ppm using tetramethylsilane (TMS) as an internal standard. NMR data was processed using Bruker TopSpin 4.0.5. FT-IR spectra were recorded on 
a Bruker ALPHA-P spectrometer using a platinum ATR with a diamond ATR crystal. Spectra are reported in terms of frequency of absorption (cm-1) and only partial data is provided where applicable. IR spectra were processed with OPUS Spectroscopy software (Version 7.5, Bruker Optik, $\mathrm{GmbH})$. Automated flash chromatography was conducted using a Biotage Isolera flash chromatography system using silica gel (60 ^, low acidity, obtained from SiliCycle, Quebec City, QC, Canada) and reagent grade solvents. Structures were drawn with ChemDraw Professional 17.1. All HRMS data was collected at the AIMS Mass Spectrometry Laboratories (University of Toronto). ESI positive mode data was collected with an Agilent 6538 UHD mass spectrometer equipped with a hybrid quadrupole time-of-flight mass analyzer (QTOF-MS).

\section{Experimental Conditions for Starting Exo-glycals and Precursors.}

\subsection{General Procedure for the Bromine Oxidation of Pentoses.}

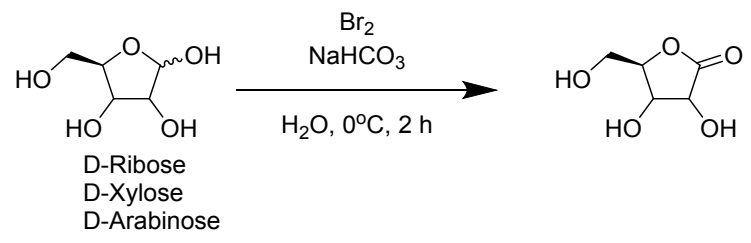

Oxidation of pentoses was performed using a modified literature procedure. ${ }^{1}$ A solution of the appropriate aldopentose ( $3.0 \mathrm{~g}, 20 \mathrm{mmol}, 1$ eq.) and $\mathrm{NaHCO}_{3}(4 \mathrm{~g}, 48 \mathrm{mmol}, 2.4$ eq.) was prepared in water $(25 \mathrm{~mL})$ and was stirred at room temperature for 15 mins. The aqueous solution was cooled to $0{ }^{\circ} \mathrm{C}$ in a salt/ice bath and was monitored by an internal thermometer. $\mathrm{Br}_{2}(1.05 \mathrm{~mL}, 20.4$ mmol, 1.02 eq.) was then added dropwise at a rate that ensured the reaction remained below $5^{\circ} \mathrm{C}$. After the complete addition of $\mathrm{Br}_{2}$, the reaction was stirred at $0{ }^{\circ} \mathrm{C}$ for $1 \mathrm{~h}$ and then at room temperature for $1 \mathrm{~h}$. The excess $\mathrm{Br}_{2}$ was blown off using a stream of pressurized air and the solvent was removed under reduced pressure to give the crude material. The crude product was then triturated with hot EtOH $(3 \times 10 \mathrm{~mL})$ and the combined extracts were concentrated under reduced pressure. The crude product was re-dissolved in a mixture of $10 \% \mathrm{MeOH}$ in EtOAc then filtered through a short silica column to yield the sugar lactone. The lactone was then used directly without further purification in general procedure 2.2.

\subsection{General Procedure for the Silylation of Carbohydrate Lactones}

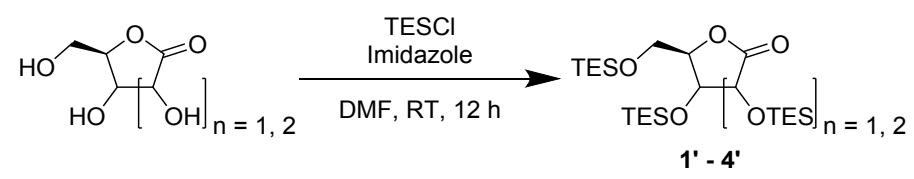

1. Cardozo, H. M.; Ribeiro, T. F.; Sa, M. M.; Sebrao, D.; Nascimento, M. G.; Silveira, G. P. J. Braz. Chem. Soc. 2015, 26(4), 755-764. 
In an oven-dried flask, a solution of the desired carbohydrate lactone was prepared under argon in anhydrous DMF (0.2 M). Imidazole (2 eq. per $\mathrm{OH}$ ) was added in one portion and the reaction was cooled to $0{ }^{\circ} \mathrm{C}$ in an ice bath. Chlorotriethylsilane (1.2 eq. per $\left.\mathrm{OH}\right)$ was added dropwise by syringe and the reaction was warmed to room temperature and continued with stirring overnight. Upon completion of the reaction, water was added to quench excess chlorotriethylsilane and the product was extracted several times from the mixture with hexanes. The combined hexane extracts were washed with water $(3 \times 20 \mathrm{~mL})$ and $\mathrm{NaCl}_{\text {(sat.) }}$ then dried over $\mathrm{MgSO}_{4}$. The solvent was removed to afford the crude product as a colorless oil. Purification was performed as indicated for each substrate below.

(1') 2,3,4,6-Tetrakis-O-(triethylsilyl)-D-glucopyrano-1,5-lactone.

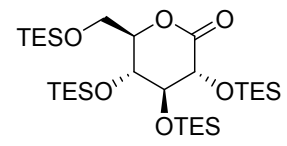

The title compound was prepared following general procedure 2.2 using commercially available D-(+)-gluconic acid $\delta$-lactone (1 g, 5.6 mmol, 1 eq.), chlorotriethylsilane (4.2 mL, 24.7 mmol, 4.4 eq.) and imidazole (3.0 g, $44.9 \mathrm{mmol}, 8$ eq.) in anhydrous DMF (25 mL). The crude compound was purified by column chromatography using $4 \%$ EtOAc in hexanes to give product 1 ' as a colorless oil (3.375 g, 95\%). Spectroscopic data is consistent with a previous report. ${ }^{2}{ }^{1} \mathrm{H}$ NMR $\left(400 \mathrm{MHz}, \mathrm{CDCl}_{3}\right) \delta 4.58-4.54(\mathrm{~m}, 1 \mathrm{H}), 4.12(\mathrm{~d}, J=3.7 \mathrm{~Hz}, 1 \mathrm{H}), 4.00(\mathrm{~d}, J=7.6 \mathrm{~Hz}, 1 \mathrm{H}), 3.89$ $(\mathrm{dd}, J=12.0,2.3 \mathrm{~Hz}, 1 \mathrm{H}), 3.88(\mathrm{~d}, J=2.3 \mathrm{~Hz}, 1 \mathrm{H}), 3.80(\mathrm{dd}, J=11.8,4.2 \mathrm{~Hz}), 0.99-0.94$ (m, $36 \mathrm{H}), 0.68-0.57(\mathrm{~m}, 24 \mathrm{H}) ;{ }^{13} \mathrm{C}\left\{{ }^{1} \mathrm{H}\right\} \mathrm{NMR}\left(100 \mathrm{MHz}, \mathrm{CDCl}_{3}\right) \delta 169.9,81.4,77.2,73.7,71.1,61.8$, $6.8,6.7,6.7,6.6,5.0,4.6,4.5$.

\section{(2') 2,3,5-Tris-O-(triethy/silyl)-D-ribofurano-1,4-lactone.}

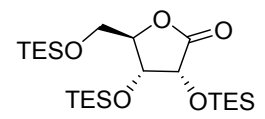

The title compound was prepared following general procedure 2.2 using D-(+)-ribono-1,4-lactone $(0.565 \mathrm{~g}, 3.8 \mathrm{mmol}, 1$ eq. ) from general procedure 2.1 , chlorotriethylsilane $(2.3 \mathrm{~mL}, 13.7 \mathrm{mmol}$, 3.6 eq.) and imidazole (1.56 g, $22.9 \mathrm{mmol}, 6$ eq.) in anhydrous DMF (20 mL). The crude compound was purified by column chromatography using $4 \%$ EtOAc in hexanes to give product $2^{\prime}$ as a colorless oil (1.1463 g, 62\%). ${ }^{1} \mathrm{H}$ NMR $\left(400 \mathrm{MHz}, \mathrm{CDCl}_{3}\right) \delta 4.60(\mathrm{~d}, J=5.1 \mathrm{~Hz}, 1 \mathrm{H}), 4.31(\mathrm{~d}, J=$ $5.1,1 \mathrm{H}), 4.27-4.26(\mathrm{~m}, 1 \mathrm{H}), 3.84(\mathrm{dd}, J=11.7,3.1 \mathrm{~Hz}, 1 \mathrm{H}), 3.78(\mathrm{dd}, J=11.6,2.2 \mathrm{~Hz}, 1 \mathrm{H}), 1.01$ $0.93(\mathrm{~m}, 27 \mathrm{H}), 0.76-0.67(\mathrm{~m}, 6 \mathrm{H}), 0.65-0.57(\mathrm{~m}, 12 \mathrm{H}) ;{ }^{13} \mathrm{C}\left\{{ }^{1} \mathrm{H}\right\} \mathrm{NMR}\left(100 \mathrm{MHz}, \mathrm{CDCl}_{3}\right) \delta 175.3$, 85.8, 72.0, 70.4, 62.1, 6.7, 6.6, 4.8, 4.1; IR (neat) $v_{\max }$ : 2954-2877 (CH, aliphatic), $1794(\mathrm{C}=\mathrm{O})$, 1459, 1414, 1147, 1004, $725 \mathrm{~cm}^{-1}$; HRMS (ESI) $\mathrm{m} / z$ : $[\mathrm{M}+\mathrm{Na}]^{+}$Calcd for $\mathrm{C}_{23} \mathrm{H}_{50} \mathrm{NaO}_{5} \mathrm{Si}_{3}$ 513.2858; found 513.2856.

2. RajanBabu, T. V.; Reddy, G. S. J. Org. Chem. 1986, 51 (26), 5458-5461. 
(3') 2,3,5-Tris-O-(triethylsilyl)-D-xylonofurano-1,4-lactone.

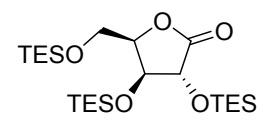

The title compound was prepared following general procedure 2.2 using D-(+)-xylono-1,4-lactone $(2.0 \mathrm{~g}, 14.9 \mathrm{mmol}, 1$ eq.) from general procedure 2.1 , chlorotriethylsilane $(9 \mathrm{~mL}, 53.5 \mathrm{mmol}, 3.6$ eq.) and imidazole $(6.0 \mathrm{~g}, 89.2 \mathrm{mmol}, 6$ eq.) in anhydrous DMF $(70 \mathrm{~mL})$. The crude compound was purified by column chromatography using $4 \%$ EtOAc in hexanes to give product $3^{\prime}$ as a colorless oil $(6.90 \mathrm{~g}, 90 \%) .{ }^{1} \mathrm{H}$ NMR $\left(400 \mathrm{MHz}, \mathrm{CDCl}_{3}\right) \delta 4.60(\mathrm{~d}, J=7.1 \mathrm{~Hz}, 1 \mathrm{H}), 4.40(\mathrm{t}, J=$ 7.1, 1H), 4.34 (ddd, $J=7.1,3.0,2.1,1 \mathrm{H}), 3.92$ (dd, $J=11.1,2.1 \mathrm{~Hz}, 1 \mathrm{H}), 3.82$ (dd, $J=11.0,3.1$ $\mathrm{Hz}, 1 \mathrm{H}), 1.00-0.93(\mathrm{~m}, 27 \mathrm{H}), 0.73-0.57(\mathrm{~m}, 18 \mathrm{H}) ;{ }^{13} \mathrm{C}\left\{{ }^{1} \mathrm{H}\right\} \mathrm{NMR}\left(100 \mathrm{MHz}, \mathrm{CDCl}_{3}\right) \delta 174.3,79.1$, 76.0, 74.0, 59.8, 6.7, 6.6, 4.9, 4.8, 4.2; IR (neat) $v_{\max }$ : 2954-2877 (CH, aliphatic), $1797(\mathrm{C}=\mathrm{O})$, 1459, 1414, 1136, 1004, $725 \mathrm{~cm}^{-1}$; HRMS (ESI) $\mathrm{m} / z$ : $[\mathrm{M}+\mathrm{Na}]^{+}$Calcd for $\mathrm{C}_{23} \mathrm{H}_{50} \mathrm{NaO}_{5} \mathrm{Si}_{3}$ 513.2858; found 513.2852.

(4') 2,3,5-Tris-O-(triethylsilyl)-D-arabinofurano-1,4-lactone.

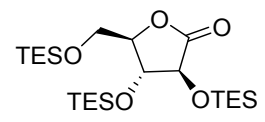

The title compound was prepared following general procedure 2.2 using D-(+)-arabino-1,4-lactone (0.8 g, $5.4 \mathrm{mmol}, 1$ eq.) from general procedure 2.1, chlorotriethylsilane ( $3.3 \mathrm{~mL}, 19.5 \mathrm{mmol}, 3.6$ eq.) and imidazole ( $2.2 \mathrm{~g}, 32.4 \mathrm{mmol}, 6$ eq.) in anhydrous DMF $(25 \mathrm{~mL})$. The crude compound was purified by column chromatography using $4 \%$ EtOAc in hexanes to give the 4 ' as a colorless oil $(2.30 \mathrm{~g}, 87 \%) .{ }^{1} \mathrm{H}$ NMR $\left(400 \mathrm{MHz}, \mathrm{CDCl}_{3}\right) \delta 4.36(\mathrm{t}, J=6.8 \mathrm{~Hz}, 1 \mathrm{H}), 4.31(\mathrm{~d}, J=7.1,1 \mathrm{H})$, 4.09-4.05 (m, 1H), $3.90(\mathrm{dd}, J=11.9,3.0 \mathrm{~Hz}, 1 \mathrm{H}), 3.77(\mathrm{dd}, J=11.9,3.6 \mathrm{~Hz}, 1 \mathrm{H}), 1.00-0.93(\mathrm{~m}$, 27H), 0.79-0.58 (m, 18H); ${ }^{13} \mathrm{C}\left\{{ }^{1} \mathrm{H}\right\}$ NMR $\left(100 \mathrm{MHz}, \mathrm{CDCl}_{3}\right) \delta 173.4,82.2,76.4,74.0,60.3,6.7$, 6.7, 6.6, 4.9, 4.8, 4.3; IR (neat) $v_{\text {max }}$ : 2954-2877 (CH, aliphatic), $1798(\mathrm{C}=\mathrm{O}), 1459,1414,1136$, 1004, $725 \mathrm{~cm}^{-1} \mathrm{HRMS}(\mathrm{ESI}) \mathrm{m} / z$ : $[\mathrm{M}+\mathrm{Na}]^{+} \mathrm{Calcd}$ for $\mathrm{C}_{23} \mathrm{H}_{50} \mathrm{NaO}_{5} \mathrm{Si}_{3} 513.2849$; found 513.2858.

\subsection{General Procedure for the Oxidation of Benzyl-Protected Carbohydrates.}

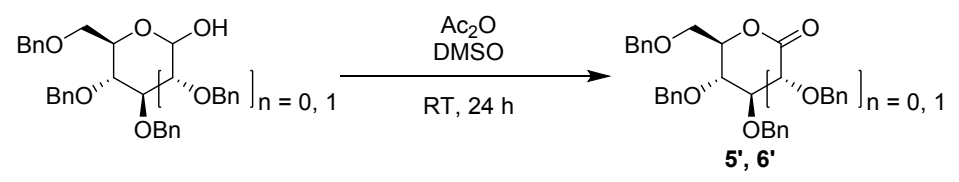

A solution of benzyl-protected carbohydrate was prepared in anhydrous DMSO. Acetic anhydride was added and the reaction was stirred for 15 hours at room temperature. The reaction was cooled to $0^{\circ} \mathrm{C}$ in an ice bath and was diluted with water. The product was extracted with EtOAc $(3 \times 20$ $\mathrm{mL})$ and the combined organic fractions were washed with $\mathrm{NaHCO}_{3 \text { (sat.) }}(3 \times 20 \mathrm{~mL})$, water $(3 \mathrm{x}$ $20 \mathrm{~mL})$ and brine $(1 \times 20 \mathrm{~mL})$. The EtOAc solution was dried over $\mathrm{MgSO}_{4}$ and the solvent was removed under reduced pressure to afford the crude product. Purification of the product was performed using column chromatography as specified below. 
(5') 2,3,4,6-Tetra-0-benzyl-D-glucono-I,5-Iactone.

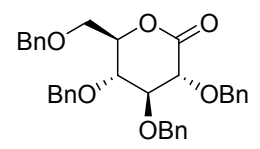

The title compound was prepared from 2,3,4,6-tetra-O-benzyl-D-glucopyranose (470 $\mathrm{mg}, 0.869$ ) mmol, DMSO $(5 \mathrm{~mL})$ and acetic anhydride $(1.6 \mathrm{~mL}, 17.4 \mathrm{mmol}, 20$ eq.) using general procedure 2.3. The product was purified by column chromatography using $30 \%$ EtOAc in hexanes to afford $5^{\prime}$ as a white solid (442.3 mg, $95 \%$ ). Spectroscopic data is consistent with a previous report. ${ }^{3}{ }^{1} \mathrm{H}$ NMR (400 MHz, $\left.\mathrm{CDCl}_{3}\right) \delta$ 7.39-7.24 (m, 18H), 7.18-7.16 (m, 2H), $4.99(\mathrm{~d}, J=11.4 \mathrm{~Hz}, 1 \mathrm{H}), 4.75-$ $4.46(\mathrm{~m}, 7 \mathrm{H}), 4.45(\mathrm{dt}, J=8.2,2.7 \mathrm{~Hz}, 1 \mathrm{H}), 4.12(\mathrm{~d}, J=6.6 \mathrm{~Hz}, 1 \mathrm{H}), 3.95(\mathrm{t}, J=7.5,1 \mathrm{H}), 3.91(\mathrm{t}$, $J=6.7 \mathrm{~Hz}, 1 \mathrm{H}), 3.72(\mathrm{dd}, J=11.0,2.4 \mathrm{~Hz}, 1 \mathrm{H}), 3.66(\mathrm{dd}, J=11.0,3.2 \mathrm{~Hz}, 1 \mathrm{H}) ;{ }^{13} \mathrm{C}\left\{{ }^{1} \mathrm{H}\right\} \mathrm{NMR}$ $\left(100 \mathrm{MHz}, \mathrm{CDCl}_{3}\right) \delta 169.3,137.6,137.5,137.5,136.9,128.5,128.4,128.4,128.1,128.0,127.9$, $127.9,127.8,81.0,78.0,77.4,76.1,73.9,73.7,73.6,68.2$.

(6') 2,3,4,6-Tetra-O-benzyl-D-galacto-1,5-Iactone

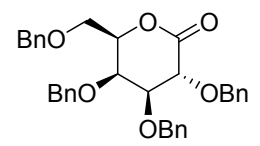

The title compound was prepared from 2,3,4,6-tetra-O-benzyl-D-galactopyranose (500 mg, 0.925) mmol, DMSO $(1.4 \mathrm{~mL})$ and acetic anhydride $(1.2 \mathrm{~mL}, 12.85 \mathrm{mmol}, 14$ eq.) using general procedure 2.3. The product was purified by column chromatography using $30 \%$ EtOAc in hexanes to afford 6 ' as a colourless oil $(425.9 \mathrm{mg}, 85 \%)$. Spectroscopic data is consistent with a previous report. ${ }^{4}{ }^{1} \mathrm{H}$ NMR $\left(400 \mathrm{MHz}, \mathrm{CDCl}_{3}\right) \delta 7.42-7.40(\mathrm{~m}, 2 \mathrm{H}), 7.36-7.21(\mathrm{~m}, 18 \mathrm{H}), 5.18(\mathrm{~d}, J=11.0$ $\mathrm{Hz}, 1 \mathrm{H}), 4.93(\mathrm{~d}, J=11.3 \mathrm{~Hz}, 1 \mathrm{H}), 4.78(\mathrm{~d}, J=11.0,1 \mathrm{H}), 4.75(\mathrm{~d}, J=11.9,1 \mathrm{H}), 4.68(\mathrm{~d}, J=11.9$ $\mathrm{Hz}, 1 \mathrm{H}), 4.60(\mathrm{~d}, J=11.3 \mathrm{~Hz}, 1 \mathrm{H}), 4.50(\mathrm{~d}, J=11.7 \mathrm{~Hz}, 1 \mathrm{H}), 4.48-4.43(\mathrm{~m}, 2 \mathrm{H}), 4.33$ (ddd, $J=$ 8.0, 5.5, $1.4 \mathrm{~Hz}, 1 \mathrm{H}), 4.16(\mathrm{t}, J=1.7 \mathrm{~Hz}, 1 \mathrm{H}), 3.88(\mathrm{dd}, J=9.5,2.1 \mathrm{~Hz}, 1 \mathrm{H}), 3.70$ (dd, $J=9.1,8.2$ $\mathrm{Hz}, 1 \mathrm{H}), 3.65(\mathrm{dd}, J=9.2,5.6 \mathrm{~Hz}, 1 \mathrm{H}) ;{ }^{13} \mathrm{C}\left\{{ }^{1} \mathrm{H}\right\} \mathrm{NMR}\left(100 \mathrm{MHz}, \mathrm{CDCl}_{3}\right) \delta 170.1,137.8,137.6$, $137.4,128.6,128.5,128.5,128.4,128.0,128.0,128.0,127.9,127.9,127.9,127.7,80.2,75.3,74.8$, $73.7,72.9,72.6,67.5$.

\section{(7') 2,3,4,6-Tetra-O-benzyl-D-manno-1,5-Iactone

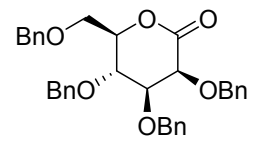

The title compound was prepared from 2,3,4,6-tetra-O-benzyl-D-mannopyranose (300 $\mathrm{mg}, 0.56$ ) mmol, DMSO $(0.8 \mathrm{~mL})$ and acetic anhydride $(0.75 \mathrm{~mL}, 7.77 \mathrm{mmol}, 14$ eq. $)$ using general procedure 2.3 . The product was purified by column chromatography using $30 \%$ EtOAc in hexanes

3. Li, T.; Guo, L.; Zhang, Y.; Wang, J.; Zhang, Z.; Li, J.; Zhang, W.; Lin, J.; Zhao, W.; Wang, P. G. Biorg. Med. Chem. 2011, 19 (7), 2136-2144.

4. Talode, J.; Kato, D.; Nagae, H.; Tsurugi, H.; Seki, M.; Mashima, K. J. Org. Chem. 2020, 85, $12382-12392$. 
to afford $7^{\prime}$ as a colourless oil ( $\left.218 \mathrm{mg}, 72 \%\right)$. Spectroscopic data is consistent with a previous report. ${ }^{5}{ }^{1} \mathrm{H}$ NMR $\left(400 \mathrm{MHz}, \mathrm{CDCl}_{3}\right) \delta 7.40-7.25(\mathrm{~m}, 18 \mathrm{H}), 7.10-7.08(\mathrm{~m}, 2 \mathrm{H}), 5.06(\mathrm{~d}, J=11.9$ $\mathrm{Hz}, 1 \mathrm{H}), 4.83(\mathrm{~d}, J=12.2 \mathrm{~Hz}, 1 \mathrm{H}), 4.63(\mathrm{~d}, J=12.2,1 \mathrm{H}), 4.59(\mathrm{~d}, J=11.9,1 \mathrm{H}), 4.53(\mathrm{~s}, 2 \mathrm{H}), 4.35$ $(\mathrm{d}, J=2.8 \mathrm{~Hz}, 1 \mathrm{H}), 4.33(\mathrm{~d}, J=12.1 \mathrm{~Hz}, 1 \mathrm{H}), 4.24(\mathrm{~d}, J=12.0 \mathrm{~Hz}, 1 \mathrm{H}), 4.23(\mathrm{~m}, 1 \mathrm{H}), 4.05(\mathrm{t}, J=$ $1.4 \mathrm{~Hz}, 1 \mathrm{H}), 3.78(\mathrm{dd}, J=7.2,1.4 \mathrm{~Hz}, 1 \mathrm{H}), 3.63(\mathrm{dd}, J=4.6 \mathrm{~Hz}, 1 \mathrm{H}) ;{ }^{13} \mathrm{C}\left\{{ }^{1} \mathrm{H}\right\} \mathrm{NMR}(100 \mathrm{MHz}$, $\left.\mathrm{CDCl}_{3}\right) \delta 169.4,137.7,137.2,136.8,128.5,128.5,128.4,128.2,128.0,128.0,127.9,127.9,127.8$, $127.8,78.6,76.7,76.1,75.5,73.5,73.0,72.9,71.8,69.1$.

(8') 2,3,5-tri-0-benzyl-D-arabino-I,4-lactone.

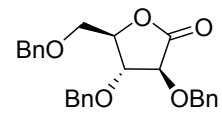

The title compound was prepared from 2,3,4,6-tetra-O-benzyl- $\beta$-D-arabinofuranose ( $500 \mathrm{mg}, 1.19$ $\mathrm{mmol})$, DMSO $(6 \mathrm{~mL})$ and acetic anhydride $(2.0 \mathrm{~mL}, 21.4 \mathrm{mmol}, 18 \mathrm{eq}$.$) using general procedure$ 2.3. The product was purified by column chromatography using $30 \%$ EtOAc in hexanes to afford 8 as a white solid (374.7 mg, $75 \%$ ). Spectroscopic data is consistent with a previous report. ${ }^{6}{ }^{1} \mathrm{H}$ NMR $\left(400 \mathrm{MHz}, \mathrm{CDCl}_{3}\right) \delta 7.41-7.20(\mathrm{~m}, 15 \mathrm{H}), 5.07(\mathrm{~d}, J=11.6 \mathrm{~Hz}, 1 \mathrm{H}), 4.77(\mathrm{~d}, J=11.6 \mathrm{~Hz}$, $1 \mathrm{H}), 4.63(\mathrm{~d}, J=11.6 \mathrm{~Hz}, 1 \mathrm{H}), 4.56(\mathrm{~d}, J=12.1 \mathrm{~Hz}, 1 \mathrm{H}), 4.52(\mathrm{~d}, J=11.6 \mathrm{~Hz}, 4.50(\mathrm{~d}, J=12.0$ $\mathrm{Hz}, 1 \mathrm{H}), 4.36-4.30(\mathrm{~m}, 3 \mathrm{H}), 3.72-3.69(\mathrm{~m}, 1 \mathrm{H}), 3.60-3.56(\mathrm{~m}, 1 \mathrm{H}) ;{ }^{13} \mathrm{C}\left\{{ }^{1} \mathrm{H}\right\} \mathrm{NMR}(100 \mathrm{MHz}$, $\left.\mathrm{CDCl}_{3}\right) \delta 172.5,137.5,137.1,136.8,128.6,128.5,128.5,128.5,128.3,128.1,127.9,127.9,127.8$, $79.3,79.2,78.9,73.5,72.7,72.5,68.0$.

\subsection{General Procedure for the Methylenation of Sugar Lactones using Tebbe's reagent.}

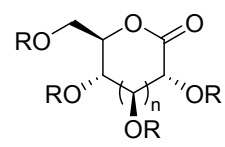
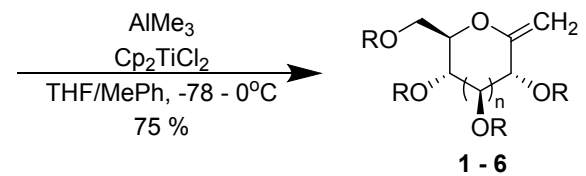

The preparation of the exo-glycals from the corresponding protected lactones was achieved following a modified procedure by Cannizzo and Grubbs. ${ }^{7}$ An oven-dried RBF equipped with a magnetic stir bar and purged with argon, was charged with bis(cyclopentadienyl)-titanium(IV) dichloride ( $2 \mathrm{mmol}, 2$ eq.). The flask was cooled to $0^{\circ} \mathrm{C}$ in an ice bath and a $2 \mathrm{M}$ solution of trimethylaluminum in anhydrous toluene under inert atmosphere $(2 \mathrm{~mL}, 4$ eq. $)$ was slowly added dropwise with gentle stirring. The resulting deep-red solution was warmed to room temperature over $2 \mathrm{~h}$ and continued with gentle stirring for $72 \mathrm{~h}$. After 72 hours another portion of $2 \mathrm{M}$ trimethylaluminum solution ( $1 \mathrm{~mL}, 2$ eq.) was added at room temperature and the reaction continued with gentle stirring for another $24 \mathrm{~h}$. The resulting Tebbe's reagent solution was then

5. Chatterjee, D.; Halder, T.; Pathak, N.; Paul, A.; Rajkamal; Yadav, S. Tetrahedr. Lett. 2017,58, 1943-1946.

6. Babu, B. S.; Balasubramanian, K. K. Carbohydr. Res. 2005, 340 (4), 753-758.

7. Cannizzo, L. F.; Grubbs, R. H. J. Org. Chem. 1985, 50 (13), 2386-2387. 
cooled to $-78{ }^{\circ} \mathrm{C}$ and a separate solution of protected sugar lactone ( $1 \mathrm{mmol}, 1$ eq.) in anhydrous THF ( $3 \mathrm{~mL}$ ) under argon was added dropwise to the Tebbe's reagent solution with gentle stirring. The reaction was warmed to $0^{\circ} \mathrm{C}$ over $3 \mathrm{~h}$ and then continued with stirring for another $2 \mathrm{~h}$ or until complete consumption of starting lactone as determined by TLC (4\% EtOAc in hexanes for silyl protected products or $30 \%$ EtOAc in hexanes for benzyl protected products). Upon completion of the reaction, a $4 \mathrm{M} \mathrm{NaOH}_{(\mathrm{aq})}$ solution was added dropwise at $0^{\circ} \mathrm{C}$ to quench the remaining trimethylaluminum and Tebbe's reagent. After complete quenching of the reaction, a thick suspension remained which was diluted with hexanes (if product is silyl protected) or EtOAc (if benzyl protected) and filtered through celite to obtain a saturated yellow filtrate. The filtrate was concentrated under reduced pressure and placed under high vacuum for $12 \mathrm{~h}$. The resulting crude product was then purified as indicated below.

(1) 3,4,5,7-Tetrakis-O-(triethylsilyl)-2,6-anhydro-1-deoxy-D-glucono-hept-1-enitol.

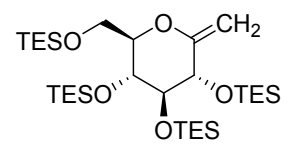

The title compound was synthesized from 1' (1.9 g, $3.0 \mathrm{mmol})$, following general procedure 2.4. The product was purified by column chromatography using $4 \%$ ethyl acetate in hexanes to give exo-glycal 1 as a pale-yellow oil $(75 \%, 1.42 \mathrm{~g})$. Spectroscopic data is consistent with a previous report. ${ }^{1} \mathrm{H}$ NMR $\left(400 \mathrm{MHz}, \mathrm{CDCl}_{3}\right) \delta 4.38(\mathrm{~s}, 1 \mathrm{H}), 4.11(\mathrm{ddd}, J=9.1,5.0,2.0 \mathrm{~Hz}, 1 \mathrm{H}), 4.02(\mathrm{~s}$, $1 \mathrm{H}), 3.99(\mathrm{~d}, J=3.0 \mathrm{~Hz}, 1 \mathrm{H}), 3.85(\mathrm{dd}, J=11.5,1.9 \mathrm{~Hz}, 1 \mathrm{H}), 3.75-3.67(\mathrm{~m}, 3 \mathrm{H}), 0.99-0.94(\mathrm{~m}$, $36 \mathrm{H}), 0.66-0.59(\mathrm{~m}, 24 \mathrm{H}) ;{ }^{13} \mathrm{C}\left\{{ }^{1} \mathrm{H}\right\} \mathrm{NMR}\left(100 \mathrm{MHz}, \mathrm{CDCl}_{3}\right) \delta 157.5,90.4,78.7,77.4,73.9,73.4$, 62.9, 6.9, 6.9, 6.8, 6.8, 5.3, 5.1, 4.9, 4.6.

\section{(2) 3,4,6-Tris-O-(triethylsilyl)-2,5-anhydro-1-deoxy-D-ribino-hex-1-enitol.}

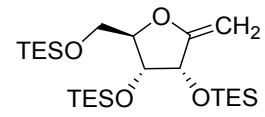

The title compound was synthesized from lactone precursor 2' (2.2 g, $4.54 \mathrm{mmol})$. The product was purified by column chromatography using 4\% ethyl acetate in hexanes to give exo-glycal 2 as a pale-yellow to colorless oil (612 mg, $36 \%) .{ }^{1} \mathrm{H}$ NMR $\left(400 \mathrm{MHz}, \mathrm{CDCl}_{3}\right) \delta 4.45(\mathrm{~d}, J=4.4 \mathrm{~Hz}$, $1 \mathrm{H}), 4.29(\mathrm{t}, J=1.4 \mathrm{~Hz}, 1 \mathrm{H}), 4.15-4.09(\mathrm{~m}, 2 \mathrm{H}), 4.02(\mathrm{t}, J=1.3 \mathrm{~Hz}, 1 \mathrm{H}), 3.77(\mathrm{dd}, J=11.5,3.3$ $\mathrm{Hz}, 1 \mathrm{H}), 3.69(\mathrm{dd}, J=11.6,3.3 \mathrm{~Hz}, 1 \mathrm{H}), 1.01-0.93(\mathrm{~m}, 27 \mathrm{H}), 0.69-0.51(\mathrm{~m}, 18 \mathrm{H}) ;{ }^{13} \mathrm{C}\left\{{ }^{1} \mathrm{H}\right\} \mathrm{NMR}$ $\left(100 \mathrm{MHz}, \mathrm{CDCl}_{3}\right) \delta 163.0,85.5,82.2,72.6,72.3,62.0,6.9,6.8,6.7,6.7,5.1,4.8,4.3$; IR (neat) $v_{\max }:$ 2954-2877 (C-H, aliphatic), 1679, 1458, 1078, 1003, $724 \mathrm{~cm}^{-1}$; HRMS (ESI) $m / z:[\mathrm{M}+\mathrm{H}]^{+}$ Calcd for $\mathrm{C}_{24} \mathrm{H}_{53} \mathrm{O}_{4} \mathrm{Si}_{3} 489.3254$; found 489.3246 .

(3) 3,4,6-Tris-O-(triethylsilyl)-2,5-anhydro-1-deoxy-D-xylono-hex-1-enitol.

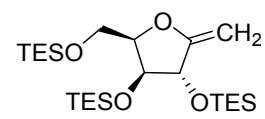

The title compound was synthesized from precursor lactone $3^{\prime}(1.0 \mathrm{~g}, 2.0 \mathrm{mmol})$. The product was purified by column chromatography using $4 \%$ ethyl acetate in hexanes to give exo-glycal 3 as a 
colorless oil (59 \%, $576.5 \mathrm{mg}) .{ }^{1} \mathrm{H}$ NMR (400 MHz, CDCl3) $\delta 4.37-4.33(\mathrm{~m}, 2 \mathrm{H}), 4.27$ (d, $J=2.3$ $\mathrm{Hz}, 1 \mathrm{H}), 4.05-4.04(\mathrm{~m}, 2 \mathrm{H}), 3.85(\mathrm{dd}, J=10.6,5.7 \mathrm{~Hz}, 1 \mathrm{H}), 3.80(\mathrm{dd}, J=10.6,5.8 \mathrm{~Hz}, 1 \mathrm{H}), 0.99-$ $0.95(\mathrm{~m}, 27 \mathrm{H}), 0.67-0.59(\mathrm{~m}, 18 \mathrm{H}) ;{ }^{13} \mathrm{C}\left\{{ }^{1} \mathrm{H}\right\}$ NMR $\left(100 \mathrm{MHz}, \mathrm{CDCl}_{3}\right) \delta 163.3,83.6,83.5,77.5$, 76.6, 60.6, 6.7, 6.7, 5.0, 4.8, 4.4; IR (neat) $v_{\max }$ : 2953-2877 (C-H, aliphatic), 1679, 1489, 1089, 1004, $724 \mathrm{~cm}^{-1}$; HRMS (ESI) $\mathrm{m} / z$ : [M+ Na] ${ }^{+}$Calcd for $\mathrm{C}_{24} \mathrm{H}_{52} \mathrm{NaO}_{4} \mathrm{Si}_{3} 511.3066$; found 511.3057.

(4) 3,4,6-Tris-O-(triethylsilyl)-2,5-anhydro-1-deoxy-D-arabino-hex-1-enitol.

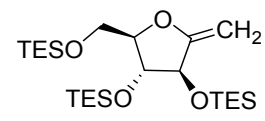

The title compound was synthesized from precursor lactone 4' (1.0 g, $2.0 \mathrm{mmol})$. The product was purified by column chromatography using $4 \%$ ethyl acetate in hexanes to give exo-glycal 4 as a pale-yellow to colorless oil (595.5 mg, $61 \%) .{ }^{1} \mathrm{H} \mathrm{NMR}\left(400 \mathrm{MHz}, \mathrm{CDCl}_{3}\right) \delta 4.37(\mathrm{~m}, 1 \mathrm{H}), 4.30$ (d, $J=2.0 \mathrm{~Hz}, 1 \mathrm{H}), 4.09-4.05(\mathrm{~m}, 3 \mathrm{H}), 3.81-3.71(\mathrm{~m}, 2 \mathrm{H}), 1.00-0.94(\mathrm{~m}, 27 \mathrm{H}), 0.68-0.57(\mathrm{~m}, 18 \mathrm{H})$; ${ }^{13} \mathrm{C}\left\{{ }^{1} \mathrm{H}\right\}$ NMR $\left(100 \mathrm{MHz}, \mathrm{CDCl}_{3}\right) \delta 163.7,87.8,83.6,78.1,77.1,62.2,6.8,6.7,6.7,4.9,4.8,4.3$; IR (neat) $v_{\max }$ : 2953-2877 (C-H, aliphatic), 1681, 1489, 1236, $724 \mathrm{~cm}^{-1}$; HRMS (ESI) $m / z:[\mathrm{M}+$ $\mathrm{Na}]^{+}$Calcd for $\mathrm{C}_{24} \mathrm{H}_{52} \mathrm{NaO}_{4} \mathrm{Si}_{3}$ 511.3066; found 511.3060.

(5) 3,4,5,7-Tetra-O-benzyl-2,6-anhydro-1-deoxy-D-gluco-hept-1-enitol.

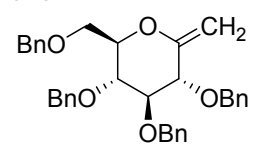

The title compound was synthesized from precursor lactone $5^{\prime}(500 \mathrm{mg}, 0.93 \mathrm{mmol})$. The product was purified by column chromatography using $30 \%$ ethyl acetate in hexanes to give exo-glycal 5 as a pale-yellow oil that solidified upon standing $(316.7 \mathrm{mg}, 63 \%)$. Spectroscopic data is consistent with a previous report. ${ }^{8}{ }^{1} \mathrm{H}$ NMR $\left(400 \mathrm{MHz}, \mathrm{CDCl}_{3}\right) \delta$ 7.38-7.25 (m, $\left.18 \mathrm{H}\right), 7.15-7.12(\mathrm{~m}, 2 \mathrm{H})$, 4.87 (d, $J=11.2 \mathrm{~Hz}, 1 \mathrm{H}), 4.79-4.75(\mathrm{~m}, 3 \mathrm{H}), 4.72(\mathrm{~d}, J=11.2 \mathrm{~Hz}, 1 \mathrm{H}), 4.66-4.23(\mathrm{~m}, 3 \mathrm{H}), 3.96$ $(\mathrm{d}, J=7.4 \mathrm{~Hz}, 1 \mathrm{H}), 3.81-3.67(\mathrm{~m}, 5 \mathrm{H}) ;{ }^{13} \mathrm{C}\left\{{ }^{1} \mathrm{H}\right\}$ NMR $\left(100 \mathrm{MHz}, \mathrm{CDCl}_{3}\right) \delta 156.4,138.4,138.1$, 138.0, 137.9, 128.5, 128.4, 128.4, 128.4, 127.9, 127.9, 127.9, 127.8, 127.7, 127.7, 94.8, 84.8, 80.0, $78.6,77.5,74.5,74.5,73.6,72.8,68.7$.

(6) 3,4,5,7-Tetra-O-benzyl-2,6-anhydro-1-deoxy-D-galacto-hept-1-enitol.

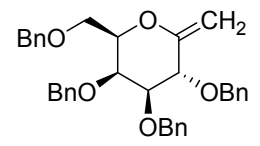

The title compound was synthesized from precursor lactone 6' $(425 \mathrm{mg}, 0.79 \mathrm{mmol})$. The product was purified by column chromatography using 30\% ethyl acetate in hexanes to give exo-glycal 6 as a yellow oil (199 $\mathrm{mg}, 47 \%)$. Spectroscopic data is consistent with that, which was previously reported. ${ }^{9}{ }^{\mathrm{H}} \mathrm{NMR}\left(400 \mathrm{MHz}, \mathrm{CDCl}_{3}\right) \delta=7.37-7.24(\mathrm{~m}, 20 \mathrm{H}), 4.93(\mathrm{~d}, J=11.5 \mathrm{~Hz}, 1 \mathrm{H}), 4.78-4.69$

8. Zhu, X.; Jin, Y.; Wickham, J. J. Org. Chem. 2007, 72 (7), 2670-2673.

9. Frédéric, C. J. M.; Cornil, J.; Vandamme, M.; Dumitrescu, L.; Tikad, A.; Robiette, R.; Vincent, S. P. Org. Lett. 2018, 20 (21), 6769-6773 
$(\mathrm{m}, 5 \mathrm{H}), 4.60(\mathrm{~d}, J=11.5 \mathrm{~Hz}, 1 \mathrm{H}), 4.52(\mathrm{~d}, J=11.9 \mathrm{~Hz}, 1 \mathrm{H}), 4.45(\mathrm{~d}, J=11.9 \mathrm{~Hz}, 1 \mathrm{H}), 4.37(\mathrm{~d}, J=9.2$ $\mathrm{Hz}, 1 \mathrm{H}), 4.06(\mathrm{t}, J=3.0 \mathrm{~Hz}, 1 \mathrm{H}), 3.81(\mathrm{td}, J=6.4,2.3 \mathrm{~Hz}, 1 \mathrm{H}), 3.71-3.60(\mathrm{~m}, 3 \mathrm{H}) ;{ }^{13} \mathrm{C}\left\{{ }^{1} \mathrm{H}\right\} \mathrm{NMR}(100$ $\left.\mathrm{MHz}, \mathrm{CDCl}_{3}\right) \delta=157.5,138.6,138.3,138.0,128.6-127.6,95.2,82.2,78.4,76.9,74.5,73.9$, 73.6, $74.5,73.1,68.6$.

(7) 3,4,5,7-Tetra-O-benzyl-2,6-anhydro-1-deoxy-D-manno-hept-1-enitol.

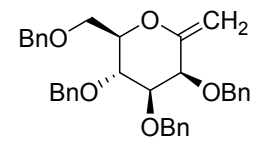

The title compound was synthesized from precursor lactone 7' (110 mg, $0.46 \mathrm{mmol})$. The product was purified by column chromatography using 30\% ethyl acetate in hexanes to give exo-glycal 7 as a colourless oil (43.5 mg, $40 \%$ ). Spectroscopic data is consistent with a previous report. ${ }^{9}{ }^{1} \mathrm{H}$ NMR $\left(400 \mathrm{MHz}, \mathrm{CDCl}_{3}\right) \delta=7.40-7.24(\mathrm{~m}, 20 \mathrm{H}), 4.93(\mathrm{~d}, J=10.8 \mathrm{~Hz}, 1 \mathrm{H}), 4.83(\mathrm{~s}, 1 \mathrm{H}), 4.77(\mathrm{~d}, J=$ $12.6 \mathrm{~Hz}, 1 \mathrm{H}), 4.67(\mathrm{~d}, J=12.1 \mathrm{~Hz}, 1 \mathrm{H}), 4.62(\mathrm{~d}, J=11.7 \mathrm{~Hz}, 1 \mathrm{H}), 4.57(\mathrm{~d}, J=11.7 \mathrm{~Hz}, 1 \mathrm{H}), 4.54(\mathrm{~d}, J=$ $11.7 \mathrm{~Hz}, 1 \mathrm{H}), 4.53(\mathrm{~d}, J=11.7 \mathrm{~Hz}, 1 \mathrm{H}), 4.43(\mathrm{~d}, J=12.6 \mathrm{~Hz}, 1 \mathrm{H}), 4.37$ (s, 1H), 4.17 (t, $J=9.2 \mathrm{~Hz}, 1 \mathrm{H})$, $4.07(\mathrm{~d}, J=3.2 \mathrm{~Hz}, 1 \mathrm{H}), 3.79(\mathrm{~m}, 2 \mathrm{H}), 3.66(\mathrm{dd}, J=9.2,3.2 \mathrm{~Hz}, 1 \mathrm{H}), 3.64-3.61(\mathrm{~m}, 1 \mathrm{H}) . ;{ }^{13} \mathrm{C}\left\{{ }^{1} \mathrm{H}\right\}$ NMR $\left(100 \mathrm{MHz}, \mathrm{CDCl}_{3}\right) \delta 154.8,138.4,138.3,138.2,138.0,128.4,128.4,128.0,127.9,127.8$, $127.7,127.6,127.6,99.7,81.5,80.2,75.1,74.1,73.6,73.5,71.4,69.4,69.3$.

(8) 3,4,6-Tri-O-benzyl-2,5-anhydro-1-deoxy-D-arabino-hex-1-enitol.

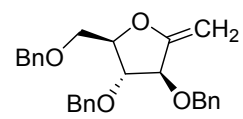

The title compound was synthesized from precursor lactone $8^{\prime}$ (375 $\left.\mathrm{mg}, 0.9 \mathrm{mmol}\right)$. The product was purified by column chromatography using $20 \%$ ethyl acetate in hexanes to give exo-glycal 8 as a colorless oil $(116.7 \mathrm{mg}, 31 \%)$. Spectroscopic data is consistent with a previous report. ${ }^{9} \mathrm{H}$ NMR $\left(400 \mathrm{MHz}, \mathrm{CDCl}_{3}\right) \delta$ 7.36-7.26 (m, 15H), $4.66(\mathrm{~d}, J=11.8 \mathrm{~Hz}, 1 \mathrm{H}), 4.59-4.50(\mathrm{~m}, 6 \mathrm{H}), 4.40$ $(\mathrm{td}, J=8.9,3.3 \mathrm{~Hz}, 1 \mathrm{H}), 4.38-4.37(\mathrm{~m}, 1 \mathrm{H}), 4.17$ (dd, $J=1.7,1.0 \mathrm{~Hz}, 1 \mathrm{H}), 4.04(\mathrm{t}, J=3.1 \mathrm{~Hz}$, $1 \mathrm{H}), 3.63(\mathrm{dd}, J=10.4,6.2 \mathrm{~Hz}, 1 \mathrm{H}), 3.59(\mathrm{dd}, J=10.5,6.1 \mathrm{~Hz}, 1 \mathrm{H}) ;{ }^{13} \mathrm{C}\left\{{ }^{1} \mathrm{H}\right\} \mathrm{NMR}(100 \mathrm{MHz}$, $\left.\mathrm{CDCl}_{3}\right) \delta 159.9,138.0,137.7,137.5,128.5,128.4,127.9,127.8,127.8,127.7,85.8,83.5,82.1$, $81.5,73.4,71.8,70.8,69.7$.

\subsection{Synthesis of 3,4,5,7-Tetra-O-benzyl-2,6-anhydro-1-deoxy-D-gluco-hept-1-enitol. (9)}

Exo-glucal (7) was prepared via a previously reported procedure. ${ }^{10}$ Tetrabutylammonium fluoride solution $(0.87 \mathrm{~mL}, 1 \mathrm{M}$ in THF) was added to a solution of previously prepared 3,4,5,7-tetra-Otriethylsilyl-2,6-anhydro-1-deoxy-D-gluco-hept-1-enitol (1) $(100 \mathrm{mg}, 0.158 \mathrm{mmol})$ in anhydrous THF $(0.8 \mathrm{~mL})$ under argon at room temperature. The resulting solution was stirred for $3 \mathrm{~h}$ at room temperature followed by the addition of pyridine $(173 \mu \mathrm{L})$ and acetic anhydride $(185 \mu \mathrm{L})$. The reaction mixture was stirred at $\mathrm{rt}$ for $24 \mathrm{~h}$. The solution was then poured into iced water $(10 \mathrm{~mL})$

10. Benltifa, M.; Hayes, J. M.; Vidal, S.; Gueyrard, D.; Goekjian, P. G.; Praly, J.-P.; Kizilis, G.; Tiraidis, C.; Alexacou, K.-M.; Chrysina, E. D.; Zographos, S. E.; Leonidas, D. D.; Archontis, G.; Oikonomakos, N. G. Biorg. Med. Chem. 2009, 17 (20), 7368-7380. 
and the product was extracted with EtOAc $(3 \times 10 \mathrm{~mL})$. The combined organic extracts were dried over $\mathrm{MgSO}_{4}$ and the solvent was removed under reduced pressure. The residue was purified by flash silica gel column chromatography (EtOAc in hexanes, $0-30 \%$ ) to afford (7) as a colourless oil (31.9 mg, 59\%). Spectroscopic data is consistent with a previous report. ${ }^{11}{ }^{1} \mathrm{H} \mathrm{NMR}(400 \mathrm{MHz}$, $\left.\mathrm{CDCl}_{3}\right) \delta 5.47(\mathrm{dt}, J=8.6,1.7 \mathrm{~Hz}, 1 \mathrm{H}), 5.22(\mathrm{t}, J=9.2 \mathrm{~Hz}, 1 \mathrm{H}), 5.16(\mathrm{t}, J=8.6 \mathrm{~Hz}, 1 \mathrm{H}), 4.84(\mathrm{t}$, $J=1.7 \mathrm{~Hz}, 1 \mathrm{H}), 4.56(\mathrm{t}, J=1.7 \mathrm{~Hz}, 1 \mathrm{H}), 4.29(\mathrm{dd}, J=12.5,4.3 \mathrm{~Hz}, 1 \mathrm{H}), 4.20(\mathrm{dd}, J=12.5,2.3$ Hz, 1H), 3.84 (ddd, $J=9.8,4.3,2.3 \mathrm{~Hz}, 1 \mathrm{H}), 2.13(\mathrm{~s}, 3 \mathrm{H}), 2.12(\mathrm{~s}, 3 \mathrm{H}), 2.05(\mathrm{~s}, 3 \mathrm{H}), 2.04(\mathrm{~s}, 3 \mathrm{H})$; ${ }^{13} \mathrm{C}\left\{{ }^{1} \mathrm{H}\right\} \mathrm{NMR}\left(100 \mathrm{MHz}, \mathrm{CDCl}_{3}\right) \delta 170.6,170.0,169.3,169.1,153.0,96.6,76.2,73.2,69.1,68.0$, $61.8,20.7,20.6,20.5$.

\section{Experimental Conditions for Aryl-Exo-Glucals.}

\subsection{General Procedure for the Arylation of Exo-Glycals.}

To a solution of exo-glycal (0.079 mmol, 1.0 eq.) in anhydrous DCE (1.2 mL) under argon at room temperature was added the specified aryl iodide (0.158 mmol, 2.0 eq.), AgOAc (0.0948 mmol, 1.2 eq. $), \mathrm{Cu}(\mathrm{OAc})_{2}(0.158 \mathrm{mmol}, 2.0$ eq. $)$, and $\mathrm{Pd}(\mathrm{OAc})_{2}(3.95 \mu \mathrm{mol}, 5.0 \mathrm{~mol} \%)$, sequentially. The reaction vial was equipped with a $3 \mathrm{ml}$ plastic syringe filled with Drierite ${ }^{\mathrm{TM}}$ and the reaction was heated in a reaction block to $70{ }^{\circ} \mathrm{C}$ with stirring for the indicated length of time. Upon completion, the reaction was allowed to cool to room temperature and was filtered. The blue-green filtrate was washed with $\mathrm{NaHCO}_{3}$ (sat.) $(3 \times 10 \mathrm{~mL})$ and water $(1 \times 10 \mathrm{~mL})$. The organic layer was dried over $\mathrm{MgSO}_{4}$ and concentrated under reduced pressure to yield the crude product as a yellow-brown oil. Isolation of the product was performed by flash chromatography with hexanes/ethyl acetate as indicated vide infra.

(1a) 3,4,5,7-Tetra-O-triethylsilyl-2,6-anhydro-1-1-deoxy-D-gluco-hept-1-enitol.

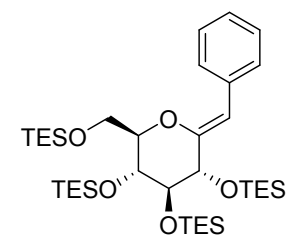

The title compound was prepared from exo-glucal 1 and iodobenzene following general procedure 2.1 for a duration of $24 \mathrm{~h}$. Purification by column chromatography using $2 \%$ ethyl acetate in hexanes afforded 1a as a pale-yellow oil $(95 \%, 53.0 \mathrm{mg}) .{ }^{1} \mathrm{H} \mathrm{NMR}\left(400 \mathrm{MHz}, \mathrm{CDCl}_{3}\right) \delta 7.65(\mathrm{~d}$, $J=7.2 \mathrm{~Hz}, 2 \mathrm{H}), 7.25(\mathrm{t}, J=7.7,2 \mathrm{H}), 7.11(\mathrm{t}, J=7.4 \mathrm{~Hz}, 1 \mathrm{H}), 5.28(\mathrm{~s}, 1 \mathrm{H}), 4.37-4.33(\mathrm{~m}, 1 \mathrm{H})$, $4.07(\mathrm{~d}, J=2.6 \mathrm{~Hz}, 1 \mathrm{H}), 3.98(\mathrm{dd}, J=11.2,2.0 \mathrm{~Hz}, 1 \mathrm{H}), 3.84-3.80(\mathrm{~m}, 2 \mathrm{H}), 3.75(\mathrm{dd}, J=9.4,2.3$ $\mathrm{Hz}, 1 \mathrm{H}), 1.00-0.94(\mathrm{~m}, 36 \mathrm{H}), 0.68-0.60(\mathrm{~m}, 24 \mathrm{H}) ; \mathrm{H}^{1}-\mathrm{H}^{3}$ nOe observed $4 \% ;{ }^{13} \mathrm{C}\left\{{ }^{1} \mathrm{H}\right\}$ NMR $(100$ $\left.\mathrm{MHz}, \mathrm{CDCl}_{3}\right) \delta 151.7,136.4,128.4,127.9,125.3,106.6,78.7,77.8,75.4,73.7,63.0,6.9,6.9,6.8$, 6.8, 5.3, 5.1, 5.0, 4.6; IR (neat) $v_{\max }: 2953-2876$ (C-H, aliphatic), 1662, 1494, 1238, 1096, 1003, $723 \mathrm{~cm}^{-1}$; HRMS (ESI) m/z: [M + Na] ${ }^{+}$Calcd for $\mathrm{C}_{37} \mathrm{H}_{72} \mathrm{NaO}_{5} \mathrm{Si}_{4} 731.4356$; found 731.4349 .

11. Tóth, M.; Kövér, K. E.; Bényei, A.; Somsák, L. Org. Biomol. Chem. 2003, 1 (22), 4039-4046. 
(1b) 3,4,5,7-Tetra-O-triethylsilyl-2,6-anhydro-1-(3-methylphenyl)-1-deoxy-D-gluco-hept-1-enitol.

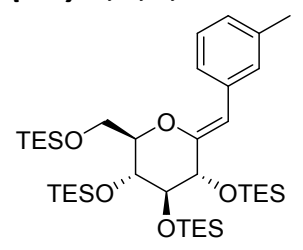

The title compound was prepared from exo-glucal 1 and 3-iodotoluene following general procedure 2.1 for a duration of $48 \mathrm{~h}$. Purification by column chromatography using $2 \%$ ethyl acetate in hexanes afforded $1 \mathrm{~b}$ as a pale-yellow oil $(84 \%, 48 \mathrm{mg}) .{ }^{1} \mathrm{H}$ NMR $\left(400 \mathrm{MHz}, \mathrm{CDCl}_{3}\right) \delta$ $7.55(\mathrm{~d}, J=7.8 \mathrm{~Hz}, 1 \mathrm{H}), 7.35(\mathrm{~s}, 1 \mathrm{H}), 7.15(\mathrm{t}, J=7.6 \mathrm{~Hz}, 1 \mathrm{H}), 6.94(\mathrm{~d}, J=7.5 \mathrm{~Hz}, 1 \mathrm{H}), 5.25$ (s, $1 \mathrm{H}), 4.36-4.32(\mathrm{~m}, 1 \mathrm{H}), 4.05(\mathrm{~d}, J=2.6 \mathrm{~Hz}, 1 \mathrm{H}), 3.97$ (dd, $J=11.2,2.0 \mathrm{~Hz}, 1 \mathrm{H}), 3.84-3.79(\mathrm{~m}$, $2 \mathrm{H}), 3.75(\mathrm{dd}, J=9.4,2.2 \mathrm{~Hz}, 1 \mathrm{H}), 2.32(\mathrm{~s}, 3 \mathrm{H}), 0.99-0.94(\mathrm{~m}, 36 \mathrm{H}), 0.67-0.59(\mathrm{~m}, 24 \mathrm{H}) ; \mathrm{H}^{1}-\mathrm{H}^{3}$ nOe observed $5 \% ;{ }^{13} \mathrm{C}\left\{{ }^{1} \mathrm{H}\right\}$ NMR $\left(100 \mathrm{MHz}, \mathrm{CDCl}_{3}\right) \delta 151.6,137.2,136.3,129.0,127.9,126.2$, $125.7,106.8,78.7,78.0,75.4,73.6,63.0,21.5,7.0,6.9,6.8,6.8,5.4,5.1,5.0,4.6$; IR (neat) $v_{\max }$ : 2953-2876 (C-H, aliphatic), 1662, 1603, 1458, 1090, 1003, $724 \mathrm{~cm}^{-1}$; HRMS (ESI) $m / z:[\mathrm{M}+\mathrm{Na}]^{+}$ Calcd for $\mathrm{C}_{38} \mathrm{H}_{74} \mathrm{NaO}_{5} \mathrm{Si}_{4} 745.4508$; found 745.4508 .

(1c) 3,4,5,7-Tetra-O-triethylsilyl-2,6-anhydro-1-(3-methoxylphenyl)-1-deoxy-D-gluco-hept-1enitol.

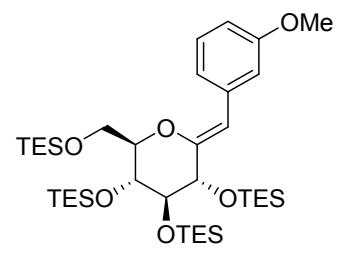

The title compound was prepared from exo-glucal 1 and 3-iodoanisole following general procedure 2.1 for a duration of $24 \mathrm{~h}$. Purification by column chromatography using $2 \%$ ethyl acetate in hexanes afforded $1 \mathrm{c}$ as a pale-yellow oil $(86 \%, 50.2 \mathrm{mg}) .{ }^{1} \mathrm{H}$ NMR $\left(400 \mathrm{MHz}, \mathrm{CDCl}_{3}\right) \delta 7.26(\mathrm{~d}$, $J=7.8 \mathrm{~Hz}, 1 \mathrm{H}), 7.22(\mathrm{~s}, 1 \mathrm{H}), 7.17(\mathrm{t}, J=7.9 \mathrm{~Hz}, 1 \mathrm{H}), 6.69(\mathrm{ddd}, J=8.1,2.6,0.8 \mathrm{~Hz}, 1 \mathrm{H}), 5.26(\mathrm{~s}$, $1 \mathrm{H}), 4.38-4.34(\mathrm{~m}, 1 \mathrm{H}), 4.06(\mathrm{~d}, J=2.6 \mathrm{~Hz}, 1 \mathrm{H}), 3.97(\mathrm{dd}, J=11.2,2.0 \mathrm{~Hz}, 1 \mathrm{H}), 3.83-3.78(\mathrm{~m}$, 2H), $3.80(\mathrm{~s}, 3 \mathrm{H}), 3.71(\mathrm{dd}, J=9.3,2.3 \mathrm{~Hz}, 1 \mathrm{H}), 0.99-0.94(\mathrm{~m}, 36 \mathrm{H}), 0.67-0.60(\mathrm{~m}, 24 \mathrm{H}) ; \mathrm{H}^{1}-\mathrm{H}^{3}$ nOe observed $3 \% ;{ }^{13} \mathrm{C}\left\{{ }^{1} \mathrm{H}\right\}$ NMR $\left(100 \mathrm{MHz}, \mathrm{CDCl}_{3}\right) \delta 159.3,152.0,138.0,128.7,121.2,113.8$, 111.1, 106.6, 78.7, 78.0, 75.4, 73.8, 63.3, 55.1, 6.9, 6.9, 6.8, 6.8, 5.4, 5.1, 5.0, 4.6; IR (neat) $v_{\max }$ : 2953-2876 (C-H, aliphatic), 1660, 1599, 1458, 1096, 1003, $724 \mathrm{~cm}^{-1}$;HRMS (ESI) $m / z:[\mathrm{M}+\mathrm{Na}]^{+}$ Calcd for $\mathrm{C}_{38} \mathrm{H}_{74} \mathrm{NaO}_{6} \mathrm{Si}_{4} 761.4457$; found 761.4455.

(1d) 3,4,5,7-Tetra-O-triethylsilyl-2,6-anhydro-1-(1-naphthyl)-1-deoxy-D-gluco-hept-1-enitol.

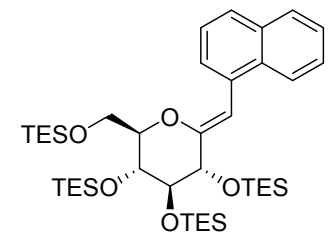


The title compound was prepared from exo-glucal 1 and 1-iodonaphthalene following general procedure 2.1 for a duration of $48 \mathrm{~h}$. Purification by column chromatography using $2 \%$ ethyl acetate in hexanes afforded $1 \mathrm{~d}$ as a pale-yellow oil $(58 \%, 34.7 \mathrm{mg}) .{ }^{1} \mathrm{H} \mathrm{NMR}\left(400 \mathrm{MHz}, \mathrm{CDCl}_{3}\right)$ $\delta 8.09-8.05(\mathrm{~m}, 2 \mathrm{H}), 7.82-7.79(\mathrm{~m}, 1 \mathrm{H}), 7.67(\mathrm{~d}, J=8.2 \mathrm{~Hz}, 1 \mathrm{H}), 7.47-7.39(\mathrm{~m}, 3 \mathrm{H}), 5.93(\mathrm{~s}, 1 \mathrm{H})$, 4.39-4.35 (m, 1H), 4.25 (d, $J=2.5 \mathrm{~Hz}, 1 \mathrm{H}), 3.91-3.88(\mathrm{~m}, 1 \mathrm{H}), 3.82(\mathrm{~m}, 2 \mathrm{H}), 3.76(\mathrm{dd}, J=11.3$, $4.9 \mathrm{~Hz}, 3 \mathrm{H}), 1.02-0.95(\mathrm{~m}, 28 \mathrm{H}), 0.86(\mathrm{t}, J=7.9,9 \mathrm{H}), 0.67-0.61(\mathrm{~m}, 18 \mathrm{H}), 0.50(\mathrm{q}, J=7.9 \mathrm{~Hz}$, $6 \mathrm{H}) ; \mathrm{H}^{1}-\mathrm{H}^{3}$ nOe observed $3 \% ;{ }^{13} \mathrm{C}\left\{{ }^{1} \mathrm{H}\right\} \mathrm{NMR}\left(100 \mathrm{MHz}, \mathrm{CDCl}_{3}\right) \delta 152.4,133.7,132.2,131.4$, 128.5, 127.1, 126.0, 125.6, 125.3, 125.0, 124.1, 102.2, 78.7, 77.7, 75.7, 73.5, 62.7, 7.0, 6.9, 6.8, 6.7, 5.4, 5.1, 5.1, 4.5; IR (neat) $v_{\max }$ : 2953-2876 (C-H, aliphatic), 1654, 1508, 1237, 1097, 1004, $723 \mathrm{~cm}^{-1}$; HRMS (ESI) $m / z$ : [M + Na] $]^{+}$Calcd for $\mathrm{C}_{41} \mathrm{H}_{74} \mathrm{NaO}_{5} \mathrm{Si}_{4}$ 781.4504; found 781.4506.

(1e) 3,4,5,7-Tetra-O-triethylsilyl-2,6-anhydro-1-(4-biphenyl)-1-deoxy-D-gluco-hept-1-enitol.

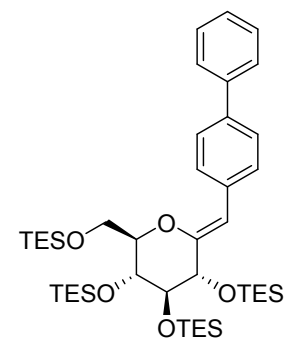

The title compound was prepared from exo-glucal 1 and 4-iodobiphenyl following general procedure 2.1 for a duration of $24 \mathrm{~h}$. Purification by column chromatography using $2 \%$ ethyl acetate in hexanes afforded 1e as a pale-yellow oil $(83 \%, 51.4 \mathrm{mg}) .{ }^{1} \mathrm{H}$ NMR $\left(400 \mathrm{MHz}, \mathrm{CDCl}_{3}\right)$ $\delta 7.73(\mathrm{~d}, J=8.4 \mathrm{~Hz}, 2 \mathrm{H}), 7.59(\mathrm{~d}, J=4.2 \mathrm{~Hz}, 2 \mathrm{H}), 7.50(\mathrm{~d}, J=8.4 \mathrm{~Hz}, 2 \mathrm{H}), 7.42$ (t, $J=5.1 \mathrm{~Hz}$, 2H), $7.31(\mathrm{t}, J=7.4 \mathrm{~Hz}, 1 \mathrm{H}), 5.33(\mathrm{~s}, 1 \mathrm{H}), 4.40-4.36(\mathrm{~m}, 1 \mathrm{H}), 4.09$ (d, $J=2.8 \mathrm{~Hz}, 1 \mathrm{H}), 4.00(\mathrm{dd}$, $J=11.2,1.9 \mathrm{~Hz}, 1 \mathrm{H}), 3.86-3.82(\mathrm{~m}, 2 \mathrm{H}), 3.76(\mathrm{dd}, J=9.4,2.3 \mathrm{~Hz}, 1 \mathrm{H}), 1.01-0.95$ (m, 36H), 0.70$0.61(\mathrm{~m}, 24 \mathrm{H}) ; \mathrm{H}^{1}-\mathrm{H}^{3}$ nOe observed $5 \% ;{ }^{13} \mathrm{C}\left\{{ }^{1} \mathrm{H}\right\} \mathrm{NMR}\left(100 \mathrm{MHz}, \mathrm{CDCl}_{3}\right) \delta 152.2,141.4,138.0$, $135.6,128.8,128.7,126.9,126.8,126.7,106.2,78.7,77.9,75.4,73.7,63.0,7.0,6.9,6.9,6.8,5.4$, 5.1, 5.0, 4.6; IR (neat) $v_{\max }: 2953-2876$ (C-H, aliphatic), 1659, 1458, 1096, 1004, $723 \mathrm{~cm}^{-1}$; HRMS (ESI) $m / z$ : $[\mathrm{M}+\mathrm{Na}]^{+}$Calcd for $\mathrm{C}_{43} \mathrm{H}_{76} \mathrm{NaO}_{5} \mathrm{Si}_{4}$ 807.4653; found 807.4662.

(1f) 3,4,5,7-Tetra-O-triethylsilyl-2,6-anhydro-1-(4-fluorophenyl)-1-deoxy-D-gluco-hept-1-enitol.

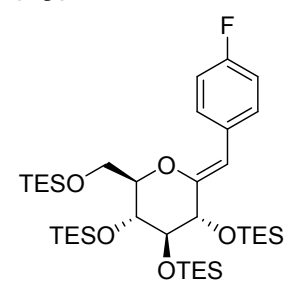

The title compound was prepared from exo-glucal 1 and 4-fluoroiodobenzene following general procedure 2.1 for a duration of $30 \mathrm{~h}$. Purification by column chromatography using $2 \%$ ethyl acetate in hexanes afforded if as a pale-yellow oil $(69 \%, 39.6 \mathrm{mg}) .{ }^{1} \mathrm{H}$ NMR $\left(400 \mathrm{MHz}, \mathrm{CDCl}_{3}\right)$ $\delta$ 7.63-7.60 (m, 2H), $6.92(\mathrm{t}, J=13.3 \mathrm{~Hz}, 2 \mathrm{H}), 5.24(\mathrm{~s}, 1 \mathrm{H}), 4.36-4.32(\mathrm{~m}, 1 \mathrm{H}), 4.04(\mathrm{~d}, J=2.6$ $\mathrm{Hz}, 1 \mathrm{H}), 3.96$ (dd, $J=11.1,2.0 \mathrm{~Hz}, 1 \mathrm{H}), 3.83-3.78$ (m, 2H), 3.73 (dd, $J=9.3,2.1 \mathrm{~Hz}, 1 \mathrm{H}), 1.00-$ 
$0.93(\mathrm{~m}, 36 \mathrm{H}), 0.67-0.59(\mathrm{~m}, 24 \mathrm{H}) ; \mathrm{H}^{1}-\mathrm{H}^{3}$ nOe observed 5\%; ${ }^{13} \mathrm{C}\left\{{ }^{1} \mathrm{H}\right\}$ NMR $\left(100 \mathrm{MHz}, \mathrm{CDCl}_{3}\right)$ $\delta 160.8(\mathrm{~d}, J=244.4 \mathrm{~Hz}), 151.3(\mathrm{~d}, J=2.1 \mathrm{~Hz}), 132.5(\mathrm{~d}, J=3.4 \mathrm{~Hz}), 129.8(\mathrm{~d}, J=7.5 \mathrm{~Hz}), 114.6$ (d, $J=21.1 \mathrm{~Hz}), 105.5,78.6,77.9,75.2,73.7,63.0,6.9,6.9,6.8,6.8,5.3,5.1,5.0,4.6$; IR (neat) $v_{\text {max }}$ : 2954-2876 (C-H, aliphatic), 1665, 1507, 1231, 1100, 1004, $723 \mathrm{~cm}^{-1}$; HRMS (ESI) $m / z$ : [M $+\mathrm{Na}]^{+}$Calcd for $\mathrm{C}_{37} \mathrm{H}_{71} \mathrm{FNaO}_{5} \mathrm{Si}_{4} 749.4249$; found 749.4255 .

(1g) 3,4,5,7-Tetra-O-triethylsilyl-2,6-anhydro-1-(4-chlorophenyl)-1-deoxy-D-gluco-hept-1-enitol.

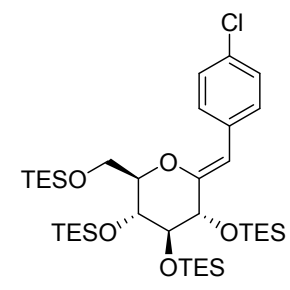

The title compound was prepared from exo-glucal 1 and 4-chloroiodobenzene following general procedure 2.1 for a duration of $24 \mathrm{~h}$. Purification by column chromatography using $2 \%$ ethyl acetate in hexanes afforded $1 \mathrm{~g}$ as a pale-yellow oil $(86 \%, 50.4 \mathrm{mg}) .{ }^{1} \mathrm{H}$ NMR $\left(400 \mathrm{MHz}, \mathrm{CDCl}_{3}\right)$ $\delta 7.58(\mathrm{~d}, J=8.9 \mathrm{~Hz}, 2 \mathrm{H}), 7.20(\mathrm{~d}, J=8.6 \mathrm{~Hz}, 2 \mathrm{H}), 5.23(\mathrm{~s}, 1 \mathrm{H}), 4.38-4.33(\mathrm{~m}, 1 \mathrm{H}), 4.05(\mathrm{~d}, J=$ $2.6 \mathrm{~Hz}, 1 \mathrm{H}), 3.97$ (dd, $J=11.2,2.0 \mathrm{~Hz}, 1 \mathrm{H}), 3.83-3.79(\mathrm{~m}, 2 \mathrm{H}), 3.74(\mathrm{dd}, J=9.3,2.0 \mathrm{~Hz}, 1 \mathrm{H})$, 1.00-0.93 (m, 36H), 0.67-0.58 (m, 24H); $\mathrm{H}^{1}-\mathrm{H}^{3}$ nOe observed $4 \% ;{ }^{13} \mathrm{C}\left\{{ }^{1} \mathrm{H}\right\}$ NMR $(100 \mathrm{MHz}$, $\left.\mathrm{CDCl}_{3}\right) \delta 152.4,134.9,130.6,129.6,128.0,105.4,78.4,78.0,75.1,73.2,62.9,6.9,6.9,6.8,6.7$, 5.3, 5.1, 5.0, 4.6; IR (neat) $v_{\max }$ : 2954-2876 (C-H, aliphatic), 1660, 1458, 1090, 1003, $724 \mathrm{~cm}^{-1}$; HRMS (ESI) $m / z$ : $[\mathrm{M}+\mathrm{Na}]^{+}$Calcd for $\mathrm{C}_{37} \mathrm{H}_{71} \mathrm{ClNaO}_{5} \mathrm{Si}_{4} 765.3963$ found 765.3959.

(1h) 3,4,5,7-Tetra-O-triethylsilyl-2,6-anhydro-1-(4-bromophenyl)-1-deoxy-D-gluco-hept-1-enitol.

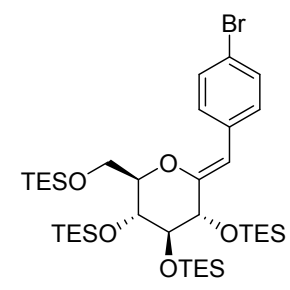

The title compound was prepared from exo-glucal 1 and 4-bromoiodobenzene following general procedure 2.1 for a duration of $24 \mathrm{~h}$. Purification by column chromatography using $2 \%$ ethyl acetate in hexanes afforded $1 \mathrm{~h}$ as a pale-yellow oil $(74 \%, 46.0 \mathrm{mg}) .{ }^{1} \mathrm{H}$ NMR $\left(400 \mathrm{MHz}, \mathrm{CDCl}_{3}\right)$ $\delta 7.52(\mathrm{~d}, J=8.5 \mathrm{~Hz}, 2 \mathrm{H}), 7.35(\mathrm{~d}, J=8.5 \mathrm{~Hz}, 2 \mathrm{H}), 5.21(\mathrm{~s}, 1 \mathrm{H}), 4.37-4.33(\mathrm{~m}, 1 \mathrm{H}), 4.04(\mathrm{~d}, J=$ $2.7 \mathrm{~Hz}, 1 \mathrm{H}), 3.96(\mathrm{dd}, J=11.2,1.9 \mathrm{~Hz}, 1 \mathrm{H}), 3.83-3.78(\mathrm{~m}, 2 \mathrm{H}), 3.74(\mathrm{dd}, J=9.3,2.1 \mathrm{~Hz}, 1 \mathrm{H})$, 1.00-0.93 (m, 36H), 0.67-0.59 (m, 24H); $\mathrm{H}^{1}-\mathrm{H}^{3}$ nOe observed $4 \% ;{ }^{13} \mathrm{C}\left\{{ }^{1} \mathrm{H}\right\}$ NMR $(100 \mathrm{MHz}$, $\left.\mathrm{CDCl}_{3}\right) \delta 152.6,135.3,130.3,129.9,118.8,105.4,78.4,78.0,75.2,73.6,63.0,6.9,6.9,6.8,6.7$, 5.3, 5.1, 5.0, 4.6; IR (neat) $v_{\max }$ : 2953-2876 (C-H, aliphatic), 1660, 1486, 1458, 1093, 1005, 724 $\mathrm{cm}^{-1}$; HRMS (ESI) $\mathrm{m} / z$ : $[\mathrm{M}+\mathrm{Na}]^{+}$Calcd for $\mathrm{C}_{37} \mathrm{H}_{71} \mathrm{BrNaO}_{5} \mathrm{Si}_{4}$ 809.3452; found 809.3454. 
(1i) 3,4,5,7-Tetra-O-triethylsilyl-2,6-anhydro-1-(3-bromophenyl)-1-deoxy-D-gluco-hept-1-enitol.

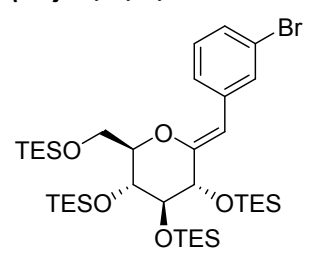

The title compound was prepared from exo-glucal 1 and 3-bromoiodobenzene following general procedure 2.1 for a duration of $48 \mathrm{~h}$. Purification by column chromatography using $2 \%$ ethyl acetate in hexanes afforded $1 \mathrm{i}$ as a pale-yellow oil $(51 \%, 31.6 \mathrm{mg}) .{ }^{1} \mathrm{H}$ NMR $\left(400 \mathrm{MHz}, \mathrm{CDCl}_{3}\right)$ $\delta 7.71(\mathrm{t}, J=1.7 \mathrm{~Hz}, 2 \mathrm{H}), 7.62(\mathrm{~d}, J=7.9 \mathrm{~Hz}, 2 \mathrm{H}), 7.23(\mathrm{~d}, J=7.9 \mathrm{~Hz}, 1 \mathrm{H}), 7.10$ (t, $J=7.9 \mathrm{~Hz}$, $1 \mathrm{H}), 5.19(\mathrm{~s}, 1 \mathrm{H}), 4.38-4.33(\mathrm{~m}, 1 \mathrm{H}), 4.05(\mathrm{~d}, J=2.7 \mathrm{~Hz}, 1 \mathrm{H}), 3.97(\mathrm{dd}, J=11.3,1.9 \mathrm{~Hz}, 1 \mathrm{H})$, 3.84-3.80 (m, 2H), 3.77 (dd, $J=9.2,1.8 \mathrm{~Hz}, 1 \mathrm{H}), 0.99-0.93(\mathrm{~m}, 36 \mathrm{H}), 0.67-0.59(\mathrm{~m}, 24 \mathrm{H}) ; \mathrm{H}^{1}-\mathrm{H}^{3}$ nOe observed $4 \% ;{ }^{13} \mathrm{C}\left\{{ }^{1} \mathrm{H}\right\}$ NMR $\left(100 \mathrm{MHz}, \mathrm{CDCl}_{3}\right) \delta 153.2,138.5,130.8,129.4,1282,126.9$, $122.1,105.1,78.3,75.1,73.4,62.9,6.9,6.9,6.8,6.8,5.3,5.1,5.0,5.0,4.6$; IR (neat) $v_{\text {max }}$ : 29532876 (C-H, aliphatic), 1660, 1590, 1458, 1096, 1003, $723 \mathrm{~cm}^{-1}$; HRMS (ESI) $m / z:[\mathrm{M}+\mathrm{Na}]^{+} \mathrm{Calcd}$ for $\mathrm{C}_{37} \mathrm{H}_{71} \mathrm{BrNaO}_{5} \mathrm{Si}_{4}$ 809.3457; found 809.3454.

(1j) 3,4,5,7-Tetra-O-triethylsilyl-2,6-anhydro-1-(3-nitrophenyl)-1-deoxy-D-gluco-hept-1-enitol.

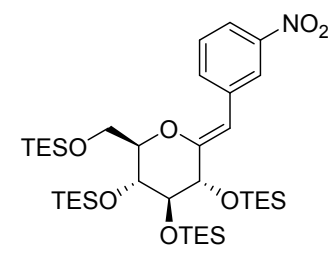

The title compound was prepared from exo-glucal 1 and 1-iodo-3-nitrobenzene following general procedure 2.1 for a duration of $24 \mathrm{~h}$. Purification by column chromatography using $2 \%$ ethyl acetate in hexanes afforded $1 J$ as a saturated yellow oil $(80 \%, 47.6 \mathrm{mg}) .{ }^{1} \mathrm{H}$ NMR $(400 \mathrm{MHz}$, $\left.\mathrm{CDCl}_{3}\right) \delta 8.28(\mathrm{t}, J=1.9 \mathrm{~Hz}, 2 \mathrm{H}), 8.13(\mathrm{~d}, J=7.9 \mathrm{~Hz}, 2 \mathrm{H}), 7.96(\mathrm{ddd}, J=8.2,2.3,0.9 \mathrm{~Hz}, 1 \mathrm{H})$, $7.39(\mathrm{t}, J=8.0 \mathrm{~Hz}, 1 \mathrm{H}), 5.33(\mathrm{~s}, 1 \mathrm{H}), 4.43-4.39(\mathrm{~m}, 1 \mathrm{H}), 4.10(\mathrm{~d}, J=2.6 \mathrm{~Hz}, 1 \mathrm{H}), 4.00(\mathrm{dd}, J=$ $11.3,2.0 \mathrm{~Hz}, 1 \mathrm{H}), 3.87-3.83(\mathrm{~m}, 2 \mathrm{H}), 3.80(\mathrm{dd}, J=9.1,1.5 \mathrm{~Hz}, 1 \mathrm{H}), 1.00-0.93(\mathrm{~m}, 36 \mathrm{H}), 0.67-$ $0.60(\mathrm{~m}, 24 \mathrm{H}) ; \mathrm{H}^{1}-\mathrm{H}^{3}$ nOe observed $4 \% ;{ }^{13} \mathrm{C}\left\{{ }^{1} \mathrm{H}\right\} \mathrm{NMR}\left(100 \mathrm{MHz}, \mathrm{CDCl}_{3}\right) \delta$ 154.6, 148.2, 138.0, 134.1, 128.7, 122.7, 119.9, 104.3, 78.6, 77.9, 74.9, 73.2, 62.9, 6.9, 6.9, 6.7, 6.7, 5.3, 5.1, 5.0, 4.5; IR (neat) $v_{\max }$ : 2954-2876 (C-H, aliphatic), 1661, 1529, 1347, 1094, 1003, $724 \mathrm{~cm}^{-1}$; HRMS (ESI) $m / z:[\mathrm{M}+\mathrm{Na}]^{+}$Calcd for $\mathrm{C}_{37} \mathrm{H}_{71} \mathrm{NNaO}_{7} \mathrm{Si}_{4}$ 776.4204; found 776.4200.

(1k) 3,4,5,7-Tetra-O-triethylsilyl-2,6-anhydro-1-(4-cyanophenyl)-1-deoxy-D-gluco-hept-1-enitol.

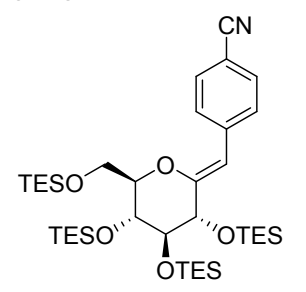


The title compound was prepared from exo-glucal 1 and 4-iodobenzonitrile following general procedure 2.1 for a duration of $24 \mathrm{~h}$. Purification by column chromatography using $2 \%$ ethyl acetate in hexanes afforded $1 \mathrm{k}$ as a pale-yellow oil $(82 \%, 47.5 \mathrm{mg}) .{ }^{1} \mathrm{H} \mathrm{NMR}\left(400 \mathrm{MHz}, \mathrm{CDCl}_{3}\right)$ $\delta 7.71(\mathrm{~d}, J=8.5 \mathrm{~Hz}, 2 \mathrm{H}), 7.58(\mathrm{~d}, J=8.5 \mathrm{~Hz}, 2 \mathrm{H}), 5.27(\mathrm{~s}, 1 \mathrm{H}), 4.43-4.39(\mathrm{~m}, 1 \mathrm{H}), 4.08(\mathrm{~d}, J=$ $2.6 \mathrm{~Hz}, 1 \mathrm{H}), 3.98$ (dd, $J=11.2,2.0 \mathrm{~Hz}, 1 \mathrm{H}), 3.85-3.80$ (m, 2H), $3.76(\mathrm{dd}, J=9.2,1.5 \mathrm{~Hz}, 1 \mathrm{H}$ ), 1.00-0.93 (m, 36H), 0.67-0.59 (m, 24H); $\mathrm{H}^{1}-\mathrm{H}^{3}$ nOe observed $4 \% ;{ }^{13} \mathrm{C}\left\{{ }^{1} \mathrm{H}\right\}$ NMR $(100 \mathrm{MHz}$, $\left.\mathrm{CDCl}_{3}\right) \delta 155.1,141.2,131.8,128.5,119.8,108.0,105.0,78.4,78.0,75.0,73.4,62.8,6.9,6.8,6.7$, 5.3, 5.1, 4.9, 4.6; IR (neat) $v_{\max }$ : 2954-2876 (C-H, aliphatic), $2223(\mathrm{CN}), 1654,1603,1093,1004$, $723 \mathrm{~cm}^{-1}$; HRMS (ESI) $\mathrm{m} / z$ : $[\mathrm{M}+\mathrm{H}]^{+}$Calcd for $\mathrm{C}_{38} \mathrm{H}_{72} \mathrm{O}_{5} \mathrm{Si}_{4} 734.4488$; found 734.4482.

(11) 3,4,5,7-Tetra-O-triethylsilyl-2,6-anhydro-1-(3-cyanophenyl)-1-deoxy-D-gluco-hept-1-enitol.

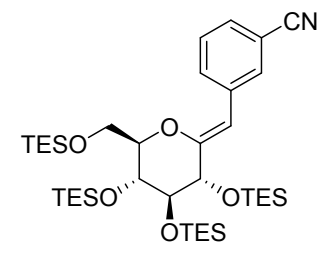

The title compound was prepared from exo-glucal 1 and 3-iodobenzonitrile following general procedure 2.1 for a duration of $24 \mathrm{~h}$. Purification by column chromatography using $2 \%$ ethyl acetate in hexanes afforded 11 as a pale-yellow oil $(87 \%, 50.4 \mathrm{mg}) .{ }^{1} \mathrm{H}$ NMR $\left(400 \mathrm{MHz}, \mathrm{CDCl}_{3}\right)$ $\delta 7.89(\mathrm{dt}, J=7.8,1.5 \mathrm{~Hz}, 1 \mathrm{H}), 7.85(\mathrm{t}, J=1.4 \mathrm{~Hz}, 1 \mathrm{H}), 7.38(\mathrm{dt}, J=7.7,1.4 \mathrm{~Hz}, 1 \mathrm{H}), 7.32(\mathrm{t}, J=$ $7.7 \mathrm{~Hz}, 1 \mathrm{H}), 5.23(\mathrm{~s}, 1 \mathrm{H}), 4.41-4.37(\mathrm{~m}, 1 \mathrm{H}), 4.07(\mathrm{~d}, J=1.5 \mathrm{~Hz}, 1 \mathrm{H}), 3.98(\mathrm{dd}, J=11.3,2.0 \mathrm{~Hz}$, $1 \mathrm{H}), 3.85-3.81$ (m, 2H), 3.78 (dd, $J=9.2,1.4 \mathrm{~Hz}, 1 \mathrm{H}), 0.99-0.94(\mathrm{~m}, 36 \mathrm{H}), 0.67-0.59$ (m, 24); $\mathrm{H}^{1}-$ $\mathrm{H}^{3}$ nOe observed $4 \% ;{ }^{13} \mathrm{C}\left\{{ }^{1} \mathrm{H}\right\} \mathrm{NMR}\left(100 \mathrm{MHz}, \mathrm{CDCl}_{3}\right) \delta 154.3,137.6,132.4,131.4,128.7,119.4$, $112.1,104.3,78.5,78.0,75.0,73.3,62.8,6.9,6.8,6.7,5.3,5.1,5.0,4.6$; IR (neat) $v_{\max }: 2953-2876$ (C-H, aliphatic), 2230 (CN), 1660, 1458, 1093, 1004, $723 \mathrm{~cm}^{-1}$; HRMS (ESI) $m / z$ : [M+ Na] ${ }^{+} \mathrm{Calcd}$ for $\mathrm{C}_{38} \mathrm{H}_{71} \mathrm{NNaO}_{5} \mathrm{Si}_{4}$ 756.4300; found 756.4300.

(1m) 3,4,5,7-Tetra-O-triethylsilyl-2,6-anhydro-1-(4-trifluorophenyl)-1-deoxy-D-gluco-hept-1enitol.

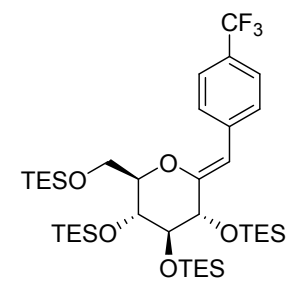

The title compound was prepared from exo-glucal 1 and 4-iodobenzotrifluoride following general procedure 2.1 for a duration of $24 \mathrm{~h}$. Purification by column chromatography using $2 \%$ ethyl acetate in hexanes afforded $1 \mathrm{~m}$ as a pale-yellow oil $(70 \%, 43.0 \mathrm{mg}) .{ }^{1} \mathrm{H} \mathrm{NMR}\left(400 \mathrm{MHz}, \mathrm{CDCl}_{3}\right)$ $\delta 7.73(\mathrm{~d}, J=8.2 \mathrm{~Hz}, 2 \mathrm{H}), 7.48(\mathrm{~d}, J=8.3 \mathrm{~Hz}, 2 \mathrm{H}), 5.30(\mathrm{~s}, 1 \mathrm{H}), 4.41-4.37(\mathrm{~m}, 1 \mathrm{H}), 4.08(\mathrm{~d}, J=$ $2.7 \mathrm{~Hz}, 1 \mathrm{H}), 3.98$ (dd, $J=11.2,2.0 \mathrm{~Hz}, 1 \mathrm{H}), 3.85-3.80(\mathrm{~m}, 2 \mathrm{H}), 3.76(\mathrm{dd}, J=9.3,1.9 \mathrm{~Hz}, 1 \mathrm{H})$, 1.00-0.94 (m, 36H), 0.67-0.59 (m, 24); $\mathrm{H}^{1}-\mathrm{H}^{3}$ nOe observed 5\%; ${ }^{13} \mathrm{C}\left\{{ }^{1} \mathrm{H}\right\}$ NMR $(100 \mathrm{MHz}$, $\left.\mathrm{CDCl}_{3}\right) \delta 154.1,139.9,128.3,126.9(\mathrm{q}, J=32.2 \mathrm{~Hz}), 124.8(\mathrm{q}, J=3.7 \mathrm{~Hz}), 124.5(\mathrm{~d}, 271.5 \mathrm{~Hz})$, 
105.2, 78.3, 75.1, 73.5, 62.9, 29.7, 6.9, 6.8, 6.7, 5.3, 5.1, 5.0, 4.6; IR (neat) $v_{\max }$ : 2954-2877 (C-H, aliphatic), 1658, 1614, 1322, 1097, 1066, 1004, $723 \mathrm{~cm}^{-1}$; HRMS (ESI) $m / z:[\mathrm{M}+\mathrm{Na}]^{+}$Calcd for $\mathrm{C}_{38} \mathrm{H}_{71} \mathrm{~F}_{3} \mathrm{NaO}_{5} \mathrm{Si}_{4}$ 799.4266; found 799.4232.

(1n) 3,4,5,7-Tetra-O-triethylsilyl-2,6-anhydro-1-(2-chloro-5-trifluorophenyl)-1-deoxy-D-glucohept-1-enitol.

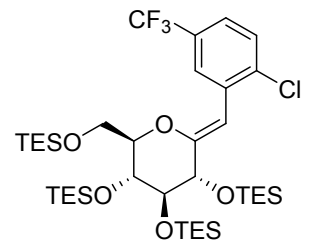

The title compound was prepared from exo-glucal 1 and 4-chloro-3-iodobenzotrifluoride following general procedure 2.1 for a duration of $24 \mathrm{~h}$. Purification by column chromatography using $2 \%$ ethyl acetate in hexanes afforded $1 \mathrm{n}$ as a pale-yellow oil $(74 \%, 47.0 \mathrm{mg}) .{ }^{1} \mathrm{H}$ NMR $(400 \mathrm{MHz}$, $\left.\mathrm{CDCl}_{3}\right) \delta 8.45(\mathrm{~d}, J=1.3 \mathrm{~Hz}, 1 \mathrm{H}), 7.41(\mathrm{~d}, J=8.3 \mathrm{~Hz}, 1 \mathrm{H}), 7.27(\mathrm{~d}, J=8.3 \mathrm{~Hz}, 1 \mathrm{H}), 5.70(\mathrm{~s}, 1 \mathrm{H})$, 4.41-4.37 (m, 1H), $4.14(\mathrm{~d}, J=3.0 \mathrm{~Hz}, 1 \mathrm{H}), 3.94(\mathrm{dd}, J=11.4,1.8 \mathrm{~Hz}, 1 \mathrm{H}), 3.86-3.79(\mathrm{~m}, 3 \mathrm{H})$, $0.97(\mathrm{t}, J=7.9 \mathrm{~Hz}, 27 \mathrm{H}), 0.90(\mathrm{t}, J=7.9 \mathrm{~Hz}, 9 \mathrm{H}), 0.68-0.61(\mathrm{~m}, 18 \mathrm{H}), 0.56(\mathrm{q}, J=7.9 \mathrm{~Hz}, 6 \mathrm{H})$; $\mathrm{H}^{1}-\mathrm{H}^{3}$ nOe observed 4\%; ${ }^{13} \mathrm{C}\left\{{ }^{1} \mathrm{H}\right\}$ NMR $\left(100 \mathrm{MHz}, \mathrm{CDCl}_{3}\right) \delta 155.0,135.2,134.6,129.3,128.8$ $(\mathrm{q}, J=32.3 \mathrm{~Hz}), 127.1(\mathrm{q}, J=3.9 \mathrm{~Hz}), 125.5,122.8(\mathrm{q}, J=3.7 \mathrm{~Hz}), 100.7,79.0,77.8,75.0,73.2$, 63.0, 6.9, 6.9, 6.7, 6.6, 5.3, 5.1, 5.0, 4.3; IR (neat) $v_{\max }$ : 2955-2877 (C-H, aliphatic), 1656, 1458, 1325, 1082, 1004, $723 \mathrm{~cm}^{-1}$; HRMS (ESI) $m / z$ : $[\mathrm{M}+\mathrm{Na}]^{+}$Calcd for $\mathrm{C}_{38} \mathrm{H}_{70} \mathrm{ClF}_{3} \mathrm{NaO}_{5} \mathrm{Si}_{4}$ 833.3836; found 833.3833 .

(10) 3,4,5,7-Tetra-O-triethylsilyl-2,6-anhydro-1-(3-trifluorophenyl)-1-deoxy-D-gluco-hept-1enitol.

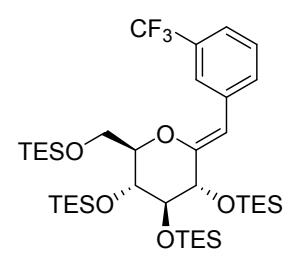

The title compound was prepared from exo-glucal 1 and 3-iodobenzotrifluoride following general procedure 2.1 for a duration of $24 \mathrm{~h}$. Purification by column chromatography using $2 \%$ ethyl acetate in hexanes afforded $1 \mathrm{o}$ as a pale-yellow oil $(70 \%, 43.0 \mathrm{mg}) .{ }^{1} \mathrm{H}$ NMR $\left(400 \mathrm{MHz}, \mathrm{CDCl}_{3}\right)$ $\delta 7.97(\mathrm{t}, J=7.6 \mathrm{~Hz}, 1 \mathrm{H}), 7.71(\mathrm{~s}, 1 \mathrm{H}), 7.35(\mathrm{~m}, 2 \mathrm{H}), 5.30(\mathrm{~s}, 1 \mathrm{H}), 4.40-4.36(\mathrm{~m}, 1 \mathrm{H}), 4.08(\mathrm{~d}, J=$ $2.9 \mathrm{~Hz}, 1 \mathrm{H}), 3.98(\mathrm{dd}, J=11.2,2.0 \mathrm{~Hz}, 1 \mathrm{H}), 3.84-3.78(\mathrm{~m}, 2 \mathrm{H}), 3.77$ (dd, $J=9.2,1.9 \mathrm{~Hz}, 1 \mathrm{H})$, 0.99-0.94 (m, 36H), 0.67-0.60 (m, 24H); $\mathrm{H}^{1}-\mathrm{H}^{3}$ nOe observed 4\%; ${ }^{13} \mathrm{C}\left\{{ }^{1} \mathrm{H}\right\}$ NMR $(100 \mathrm{MHz}$, $\left.\mathrm{CDCl}_{3}\right) \delta 153.5,137.1,131.4,130.2(\mathrm{q}, J=31.6 \mathrm{~Hz}), 128.3,124.7(\mathrm{q}, J=3.7 \mathrm{~Hz}), 124.4(\mathrm{~d}, J=$ $272.3 \mathrm{~Hz}), 121.8(\mathrm{q}, J=3.7 \mathrm{~Hz}), 105.2,78.4,78.2,75.1,73.4,63.0,6.9,6.9,6.7,5.3,5.1,5.0,4.5$; IR (neat) $v_{\text {max }}$ : 2954-2876 (C-H, aliphatic), 1663, 1458, 1327, 1097, 1003, $724 \mathrm{~cm}^{-1}$; HRMS (ESI) $m / z:[\mathrm{M}+\mathrm{Na}]^{+}$Calcd for $\mathrm{C}_{38} \mathrm{H}_{71} \mathrm{~F}_{3} \mathrm{NaO}_{5} \mathrm{Si}_{4} 799.4221$; found 799.4223. 
(1p) 3,4,5,7-Tetra-O-triethylsilyl-2,6-anhydro-1-(4-(hydroxymethyl)-phenyl)-1-deoxy-D-glucohept-1-enitol.

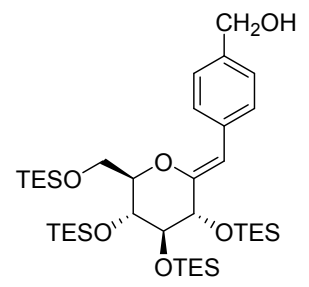

The title compound was prepared from exo-glucal 1 and 4-iodobenzyl alcohol following general procedure 2.1 for a duration of $24 \mathrm{~h}$. Purification by column chromatography using $4 \%$ ethyl acetate in hexanes afforded $1 \mathrm{p}$ as a pale-yellow oil $(81 \%, 47.2 \mathrm{mg}) .{ }^{1} \mathrm{H} \mathrm{NMR}\left(400 \mathrm{MHz}, \mathrm{CDCl}_{3}\right)$ $\delta 7.65(\mathrm{~d}, J=8.2 \mathrm{~Hz}, 2 \mathrm{H}), 7.25(\mathrm{~d}, J=8.1 \mathrm{~Hz}, 2 \mathrm{H}), 5.28(\mathrm{~s}, 1 \mathrm{H}), 4.64(\mathrm{~s}, 2 \mathrm{H}), 4.38-4.34(\mathrm{~m}, 1 \mathrm{H})$, 4.07 (d, $J=2.7 \mathrm{~Hz}, 1 \mathrm{H}), 3.98$ (dd, $J=11.1,1.8 \mathrm{~Hz}, 1 \mathrm{H}), 3.83-3.79$ (m, 2H), 3.75 (dd, $J=9.3,2.2$ $\mathrm{Hz}, 1 \mathrm{H}), 1.59(\mathrm{~s}, 1 \mathrm{H}), 1.00-0.93(\mathrm{~m}, 36 \mathrm{H}), 0.68-0.59(\mathrm{~m}, 24 \mathrm{H}) ; \mathrm{H}^{1}-\mathrm{H}^{3}$ nOe observed $4 \% ;{ }^{13} \mathrm{C}\left\{{ }^{1} \mathrm{H}\right\}$ NMR $\left(100 \mathrm{MHz}, \mathrm{CDCl}_{3}\right) \delta 152.0,137.8,136.0,128.6,126.9,106.2,78.6,77.9,75.3,65.5,63.0$, 6.9, 6.9, 6.8, 6.8, 5.3, 5.1 , 5.0, 4.6; IR (neat) $v_{\max }: 3400(\mathrm{O}-\mathrm{H}), 2953-2876$ (C-H, aliphatic), 1662, 1096, 1003, $723 \mathrm{~cm}^{-1}$; HRMS (ESI) $\mathrm{m} / z$ : [M + Na] ${ }^{+}$Calcd for $\mathrm{C}_{38} \mathrm{H}_{74} \mathrm{NaO}_{6} \mathrm{Si}_{4} 761.4464$; found 761.4455 .

(1q) 3,4,5,7-Tetra-O-triethylsilyl-2,6-anhydro-1-(3-(hydroxymethyl)-phenyl)-1-deoxy-D-glucohept-1-enitol.

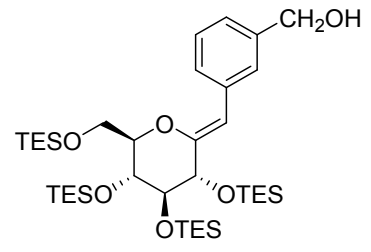

The title compound was prepared from exo-glucal 1 and 3-iodobenzyl alcohol following general procedure 2.1 for a duration of $24 \mathrm{~h}$. Purification by column chromatography using $4 \%$ ethyl acetate in hexanes afforded 1q as a pale-yellow oil $(86 \%, 47.2 \mathrm{mg}) .{ }^{1} \mathrm{H} \mathrm{NMR}\left(400 \mathrm{MHz}, \mathrm{CDCl}_{3}\right)$ $\delta 7.73(\mathrm{~d}, J=7.8 \mathrm{~Hz}, 1 \mathrm{H}), 7.48(\mathrm{~s}, 1 \mathrm{H}), 7.25$ (t, $J=11.5 \mathrm{~Hz}, 1 \mathrm{H}), 7.14(\mathrm{~d}, J=7.7 \mathrm{~Hz}, 1 \mathrm{H}), 5.29$ $(\mathrm{s}, 1 \mathrm{H}), 4.66(\mathrm{~d}, J=4.7 \mathrm{~Hz}, 2 \mathrm{H}), 4.38-4.34(\mathrm{~m}, 1 \mathrm{H}), 4.07(\mathrm{~d}, J=2.7 \mathrm{~Hz}, 1 \mathrm{H}), 3.98(\mathrm{dd}, J=11.2$, $2.0 \mathrm{~Hz}, 1 \mathrm{H}), 3.84-3.80(\mathrm{~m}, 2 \mathrm{H}), 3.74(\mathrm{dd}, J=9.3,2.0 \mathrm{~Hz}, 1 \mathrm{H}), 1.66$ (t, $J=4.8 \mathrm{~Hz}, 1 \mathrm{H}), 0.99-0.94$ $(\mathrm{m}, 36 \mathrm{H}), 0.67-0.60(\mathrm{~m}, 24 \mathrm{H}) ; \mathrm{H}^{1}-\mathrm{H}^{3}$ nOe observed 4\%; ${ }^{13} \mathrm{C}\left\{{ }^{1} \mathrm{H}\right\}$ NMR $\left(100 \mathrm{MHz}, \mathrm{CDCl}_{3}\right) \delta$ 152.1, 140.4, 136.7, 128.3, 127.9, 126.9, 124.1, 106.4, 78.5, 78.0, 75.3, 73.6, 65.7, 63.1, 6.9, 6.9, 6.8, 6.8, 5.3, 5.1, 5.0, 4.6; IR (neat) $v_{\max }: 3350(\mathrm{OH}), 2953-2876(\mathrm{C}-\mathrm{H}$, aliphatic), 1662, 1458, 1096, 1003, $723 \mathrm{~cm}^{-1}$; HRMS (ESI) $m / z$ : $[\mathrm{M}+\mathrm{Na}]^{+}$Calcd for $\mathrm{C}_{38} \mathrm{H}_{74} \mathrm{NaO}_{6} \mathrm{Si}_{4} 761.4455$; found 761.4446 . 
(1r) 3,4,5,7-Tetra-O-triethylsilyl-2,6-anhydro-1-(3-(hydroxy(phenyl)methyl)phenyl)-1-deoxy-Dgluco-hept-1-enitol.

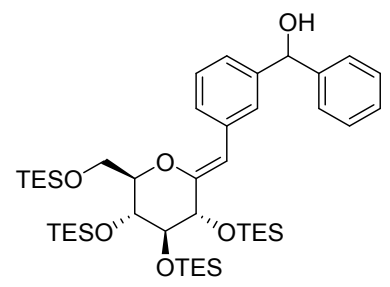

The title compound was prepared from exo-glucal 1 and (3-iodophenyl)(phenyl)methanol following general procedure 2.1 for a duration of $24 \mathrm{~h}$. Purification by column chromatography using $10 \%$ ethyl acetate in hexanes afforded $1 \mathrm{r}$ as a pale-yellow oil $(76 \%, 49.0 \mathrm{mg}) .{ }^{1} \mathrm{H}$ NMR $\left(400 \mathrm{MHz}, \mathrm{CDCl}_{3}\right) \delta 7.78(\mathrm{t}, J=10.5 \mathrm{~Hz}, 1 \mathrm{H}), 7.46(\mathrm{~s}, 1 \mathrm{H}), 7.40(\mathrm{~d}, J=8.0 \mathrm{~Hz}, 2 \mathrm{H}), 7.32(\mathrm{t}, J=$ $7.6 \mathrm{~Hz}, 2 \mathrm{H}), 7.23(\mathrm{q}, J=7.4 \mathrm{~Hz}, 2 \mathrm{H}), 7.10-7.08(\mathrm{~m}, 1 \mathrm{H}), 5.81(\mathrm{~s}, 1 \mathrm{H}), 5.29(\mathrm{~s}, 1 \mathrm{H}), 4.37-4.32(\mathrm{~m}$, $1 \mathrm{H}), 4.05(\mathrm{~d}, J=2.9 \mathrm{~Hz}, 1 \mathrm{H}), 3.96(\mathrm{dt}, J=9.4,1.8 \mathrm{~Hz}, 1 \mathrm{H}), 3.83-3.77(\mathrm{~m}, 2 \mathrm{H}), 3.71(\mathrm{dt}, J=9.3$, $3.0 \mathrm{~Hz}, 1 \mathrm{H}), 2.21(\mathrm{~s}, 1 \mathrm{H}), 0.99-0.94(\mathrm{~m}, 36 \mathrm{H}), 0.67-0.60(\mathrm{~m}, 24 \mathrm{H}) ; \mathrm{H}^{1}-\mathrm{H}^{3}$ nOe observed 5\%; ${ }^{13} \mathrm{C}\left\{{ }^{1} \mathrm{H}\right\} \operatorname{NMR}\left(100 \mathrm{MHz}, \mathrm{CDCl}_{3}\right) \delta 152.1,143.9,143.4,143.3,136.6,136.5,128.4,128.3,128.2$, 127.9, 127.4, 126.6., 126.6, 126.5, 123.7, 123.6, 106.5, 78.7, 78.6, 78.0, 76.5, 75.3, 73.7, 63.2, 7.0, 6.9, 6.8, 6.8, 5.4, 5.1, 5.0, 4.6; IR (neat) $v_{\max }: 3400(\mathrm{O}-\mathrm{H}), 2953-2876$ (C-H, aliphatic), 1662, 1599, 1457, 1097, 1003, $724 \mathrm{~cm}^{-1}$; HRMS (ESI) $m / z$ : $[\mathrm{M}+\mathrm{Na}]^{+}$Calcd for $\mathrm{C}_{44} \mathrm{H}_{78} \mathrm{NaO}_{6} \mathrm{Si}_{4} 837.4778$; found 837.4768 .

(1s) 3,4,5,7-Tetra-O-triethylsilyl-2,6-anhydro-1-(3-formylphenyl)-1-deoxy-D-gluco-hept-1-enitol.

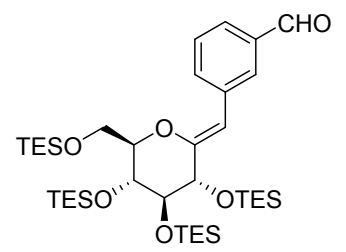

The title compound was prepared from exo-glucal 1 and 3-iodobenzaldehyde following general procedure 2.1 for a duration of $24 \mathrm{~h}$. Purification by column chromatography using $2 \%$ ethyl acetate in hexanes afforded $1 \mathrm{~s}$ as a pale-yellow oil $(77 \%, 44.8 \mathrm{mg}) .{ }^{1} \mathrm{H} \mathrm{NMR}\left(400 \mathrm{MHz}, \mathrm{CDCl}_{3}\right)$ $\delta 9.99(\mathrm{~s}, 1 \mathrm{H}), 8.07(\mathrm{~d}, J=7.8 \mathrm{~Hz}, 1 \mathrm{H}), 7.97(\mathrm{~s}, 1 \mathrm{H}), 7.64(\mathrm{~d}, J=7.6 \mathrm{~Hz}, 1 \mathrm{H}), 7.41(\mathrm{t}, J=7.7 \mathrm{~Hz}$, $1 \mathrm{H}), 5.34(\mathrm{~s}, 1 \mathrm{H}), 4.41-4.38(\mathrm{~m}, 1 \mathrm{H}), 4.10(\mathrm{~d}, J=2.7 \mathrm{~Hz}, 1 \mathrm{H}), 3.99(\mathrm{dd}, J=11.2,1.5 \mathrm{~Hz}, 1 \mathrm{H})$, 3.85-3.81 (m, 2H), 3.77 (dd, $J=9.2,1.4 \mathrm{~Hz}, 1 \mathrm{H}), 0.99-0.94(\mathrm{~m}, 36 \mathrm{H}), 0.67-0.60(\mathrm{~m}, 24 \mathrm{H}) ; \mathrm{H}^{1}-\mathrm{H}^{3}$ nOe observed $4 \% ;{ }^{13} \mathrm{C}\left\{{ }^{1} \mathrm{H}\right\}$ NMR $\left(100 \mathrm{MHz}, \mathrm{CDCl}_{3}\right) \delta 193.0,153.4,137.4,136.3,134.2,130.1$, $128.6,126.1,105.2,78.3,78.2,75.1,63.0,6.9,6.9,6.8,5.3,5.1,5.0,4.6$; IR (neat) $v_{\max }$ : 29542876 (C-H, aliphatic), 1701 (C=O, aldehyde), 1661, 1458, 1096, 1003, $724 \mathrm{~cm}^{-1}$; HRMS (ESI) $m / z:[\mathrm{M}+\mathrm{Na}]^{+}$Calcd for $\mathrm{C}_{38} \mathrm{H}_{72} \mathrm{NaO}_{6} \mathrm{Si}_{4} 759.4300$; found 759.4298 . 
(1t) 3,4,5,7-Tetra-O-triethylsilyl-2,6-anhydro-1-(4-formylphenyl)-1-deoxy-D-gluco-hept-1-enitol.

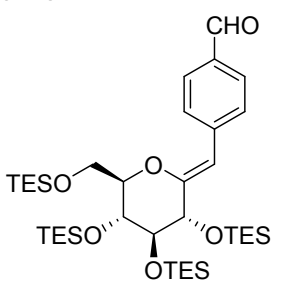

The title compound was prepared from exo-glucal 1 and 4-iodobenzaldehyde following general procedure 2.1 for a duration of $24 \mathrm{~h}$. Purification by column chromatography using $2 \%$ ethyl acetate in hexanes afforded $1 \mathrm{t}$ as a pale-yellow oil $(64 \%, 37.2 \mathrm{mg}) .{ }^{1} \mathrm{H}$ NMR $\left(400 \mathrm{MHz}, \mathrm{CDCl}_{3}\right)$ $\delta 9.93(\mathrm{~s}, 1 \mathrm{H}), 7.80-7.74(\mathrm{~m}, 4 \mathrm{H}), 5.33(\mathrm{~s}, 1 \mathrm{H}), 4.45-4.40(\mathrm{~m}, 1 \mathrm{H}), 4.10(\mathrm{~d}, J=2.8 \mathrm{~Hz}, 1 \mathrm{H}), 4.00$ $(\mathrm{dd}, J=11.2,1.9 \mathrm{~Hz}, 1 \mathrm{H}), 3.86-3.82(\mathrm{~m}, 2 \mathrm{H}), 3.79(\mathrm{dd}, J=9.2,1.7 \mathrm{~Hz}, 1 \mathrm{H}), 1.01-0.94(\mathrm{~m}, 36 \mathrm{H})$, 0.69-0.60 (m, 24H); $\mathrm{H}^{1}-\mathrm{H}^{3}$ nOe observed 4\%; ${ }^{13} \mathrm{C}\left\{{ }^{1} \mathrm{H}\right\}$ NMR $\left(100 \mathrm{MHz}, \mathrm{CDCl}_{3}\right) \delta 191.8,155.4$, 143.0, 133.4, 129.7, 128.5, 105.6, 78.4, 78.1, 75.1, 73.4, 62.8, 6.9, 6.8, 6.7, 5.3, 5.1, 5.0, 4.6; IR (neat) $v_{\text {max }}$ : 2953-2876 (C-H, aliphatic), 1699 (C=O, aldehyde), 1656, 1599, 1458, 1091, 1003, 724 $\mathrm{cm}^{-1}$; HRMS (ESI) $m / z$ : [M + Na $]^{+}$Calcd for $\mathrm{C}_{38} \mathrm{H}_{72} \mathrm{NaO}_{6} \mathrm{Si}_{4}$ 759.4297; found 759.4298.

(1u) 3,4,5,7-Tetra-O-triethylsilyl-2,6-anhydro-1-(4-carboxyphenyl)-1-deoxy-D-gluco-hept-1enitol.

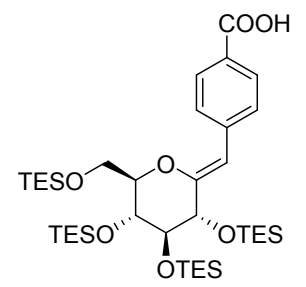

The title compound was prepared from exo-glucal 1 and 4-iodobenzoic acid following general procedure 2.1 for a duration of $24 \mathrm{~h}$. Purification by column chromatography using $10 \%$ ethyl acetate in hexanes afforded $1 \mathrm{u}$ as a pale-yellow oil $(60 \%, 35.6 \mathrm{mg}) .{ }^{1} \mathrm{H} \mathrm{NMR}\left(400 \mathrm{MHz}, \mathrm{CDCl}_{3}\right)$ $\delta 7.99(\mathrm{~d}, J=8.4 \mathrm{~Hz}, 2 \mathrm{H}), 7.72(\mathrm{~d}, J=8.5 \mathrm{~Hz}, 2 \mathrm{H}), 5.33(\mathrm{~s}, 1 \mathrm{H}), 4.43-4.39(\mathrm{~m}, 1 \mathrm{H}), 4.09(\mathrm{~d}, J=$ $2.8 \mathrm{~Hz}, 1 \mathrm{H}), 4.00(\mathrm{dd}, J=11.5,1.9 \mathrm{~Hz}, 1 \mathrm{H}), 3.86-3.82(\mathrm{~m}, 2 \mathrm{H}), 3.78(\mathrm{dd}, J=9.3,1.8 \mathrm{~Hz}, 1 \mathrm{H})$, 1.01-0.94 (m, 36H), 0.69-0.60 (m, 24H); $\mathrm{H}^{1}-\mathrm{H}^{3}$ nOe observed 1\%; ${ }^{13} \mathrm{C}\left\{{ }^{1} \mathrm{H}\right\}$ NMR $(100 \mathrm{MHz}$, $\left.\mathrm{CDCl}_{3}\right) \delta 154.8,142.1,130.0,128.0,105.7,78.3,75.2,73.4,62.8,29.7,6.9,6.8,6.7,5.3,5.1,5.0$, 4.6; HRMS (ESI) $m / z$ : [M+Na] $]^{+}$Calcd for $\mathrm{C}_{38} \mathrm{H}_{72} \mathrm{NaO}_{7} \mathrm{Si}_{4} 775.4200$; found 775.4300.

(1v) 3,4,5,7-Tetra-O-triethylsilyl-2,6-anhydro-1-(3-(methoxycarbonyl)phenyl)-1-deoxy-D-glucohept-1-enitol.

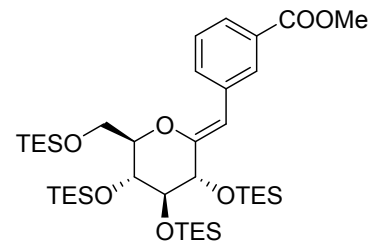


The title compound was prepared from exo-glucal 1 and 3-iodomethylbenzoate following general procedure 2.1 for a duration of $24 \mathrm{~h}$. Purification by column chromatography using $2 \%$ ethyl acetate in hexanes afforded $1 \mathrm{v}$ as a pale-yellow oil $(91 \%, 55.1 \mathrm{mg}) .{ }^{1} \mathrm{H} \mathrm{NMR}\left(400 \mathrm{MHz}, \mathrm{CDCl}_{3}\right)$ $\delta 8.19(\mathrm{~d}, J=7.9 \mathrm{~Hz}, 1 \mathrm{H}), 7.94(\mathrm{~s}, 1 \mathrm{H}), 7.78(\mathrm{~d}, J=7.9 \mathrm{~Hz}, 1 \mathrm{H}), 7.32(\mathrm{t}, J=7.8 \mathrm{~Hz}, 1 \mathrm{H}), 5.33(\mathrm{~s}$, $1 \mathrm{H}), 4.38-4.34(\mathrm{~m}, 1 \mathrm{H}), 4.08(\mathrm{~d}, J=2.9 \mathrm{~Hz}, 1 \mathrm{H}), 3.98(\mathrm{dd}, J=11.2,1.9 \mathrm{~Hz}, 1 \mathrm{H}), 3.91(\mathrm{~s}, 3 \mathrm{H})$, $3.86-3.81(\mathrm{~m}, 2 \mathrm{H}), 3.78(\mathrm{dd}, J=9.2,2.0 \mathrm{~Hz}, 1 \mathrm{H}), 0.99-0.93(\mathrm{~m}, 36 \mathrm{H}), 0.67-0.60(\mathrm{~m}, 24 \mathrm{H}) ; \mathrm{H}^{1}-\mathrm{H}^{3}$ nOe observed 5\%; ${ }^{13} \mathrm{C}\left\{{ }^{1} \mathrm{H}\right\}$ NMR $\left(100 \mathrm{MHz}, \mathrm{CDCl}_{3}\right) \delta 167.5,152.9,136.7,132.8,129.7,128.1$, $126.4,105.7,78.4,78.2,75.2,73.4,62.9,52.0,6.9,6.9,6.8,5.3,5.1,5.0,5.0,4.5$; IR (neat) $v_{\max }$ : 2953-2876 (C-H, aliphatic), 1726 (C=O, ester), 1661, 1458, 1278, 1100, 1003, $724 \mathrm{~cm}^{-1}$; HRMS (ESI) $m / z:[\mathrm{M}+\mathrm{Na}]^{+}$Calcd for $\mathrm{C}_{39} \mathrm{H}_{74} \mathrm{NaO}_{7} \mathrm{Si}_{4}$ 789.4401; found 789.4404 .

(1w) 3,4,5,7-Tetra-O-triethylsilyl-2,6-anhydro-1-(4-(Methoxycarbonyl)phenyl)-1-deoxy-D-glucohept-1-enitol.

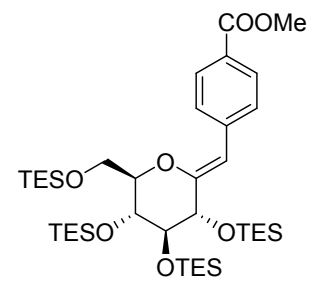

The title compound was prepared from exo-glucal 1 and 4-iodomethylbenzoate following general procedure 2.1 for a duration of $24 \mathrm{~h}$. Purification by column chromatography using $4 \%$ ethyl acetate in hexanes afforded $1 \mathrm{w}$ as a pale yellow oil $(91 \%, 55.0 \mathrm{mg}) .{ }^{1} \mathrm{H} \mathrm{NMR}\left(400 \mathrm{MHz}, \mathrm{CDCl}_{3}\right)$ $\delta 7.95(\mathrm{~d}, J=8.4 \mathrm{~Hz}, 2 \mathrm{H}), 7.72(\mathrm{~d}, J=8.4 \mathrm{~Hz}, 2 \mathrm{H}), 5.34(\mathrm{~s}, 1 \mathrm{H}), 4.45-4.41(\mathrm{~m}, 1 \mathrm{H}), 4.12(\mathrm{~d}, J=$ $2.7 \mathrm{~Hz}, 1 \mathrm{H}), 4.02(\mathrm{dd}, J=11.2,1.9 \mathrm{~Hz}, 1 \mathrm{H}), 3.92(\mathrm{~s}, 3 \mathrm{H}), 3.88-3.84(\mathrm{~m}, 2 \mathrm{H}), 3.81(\mathrm{dd}, 9.3,1.9 \mathrm{~Hz}$, $1 \mathrm{H}), 1.04-0.97(\mathrm{~m}, 36 \mathrm{H}), 0.71-0.62(\mathrm{~m}, 24 \mathrm{H}) ; \mathrm{H}^{1}-\mathrm{H}^{3}$ nOe observed $5 \% ;{ }^{13} \mathrm{C}\left\{{ }^{1} \mathrm{H}\right\}$ NMR $(100 \mathrm{MHz}$, $\left.\mathrm{CDCl}_{3}\right) \delta 167.3,154.3,141.2,129.3,128.0,126.6,105.8,78.3,75.2,73.4,62.9,51.9,6.9,6.8,6.7$, 5.3, 5.1, 5.0, 4.6; IR (neat) $v_{\max }$ : 2953-2876 (C-H, aliphatic), 1722 (C=O, ester), 1656, 1505, 1458, 1275, 1098, 1004, $724 \mathrm{~cm}^{-1}$; HRMS (ESI) $m / z$ : $[\mathrm{M}+\mathrm{Na}]^{+}$Calcd for $\mathrm{C}_{39} \mathrm{H}_{74} \mathrm{NaO}_{7} \mathrm{Si}_{4} 789.4396$; found 789.4404 .

(2a) 3,4,6-Tri-O-triethylsilyl-2,5-anhydro-1-phenyl-1-deoxy-D-ribino-hex-1-enitol.

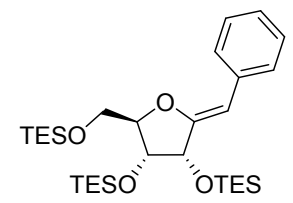

The title compound was prepared from exo-ribal 2 and iodobenzene following general procedure 2.1 for a duration of $24 \mathrm{~h}$. Purification by column chromatography using $2 \%$ ethyl acetate in hexanes afforded $2 \mathrm{a}$ as a colourless oil $(60 \%, 30.6 \mathrm{mg}) .{ }^{1} \mathrm{H}$ NMR $\left(400 \mathrm{MHz}, \mathrm{CDCl}_{3}\right) \delta 7.55(\mathrm{dd}$, $J=8.4,1.2 \mathrm{~Hz}, 2 \mathrm{H}), 7.24(\mathrm{t}, J=11.5 \mathrm{~Hz}, 2 \mathrm{H}), 7.09(\mathrm{t}, J=7.4 \mathrm{~Hz}, 1 \mathrm{H}), 5.33(\mathrm{~s}, 1 \mathrm{H}), 4.54(\mathrm{~d}, J=$ $4.3 \mathrm{~Hz}, 1 \mathrm{H}), 4.37-4.34(\mathrm{~m}, 1 \mathrm{H}), 4.18(\mathrm{t}, J=4.8 \mathrm{~Hz}, 1 \mathrm{H}), 3.93(\mathrm{dd}, J=11.7,3.1 \mathrm{~Hz}, 1 \mathrm{H}), 3.78(\mathrm{dd}$, $J=11.7,3.3 \mathrm{~Hz}, 1 \mathrm{H}), 1.02-0.91(\mathrm{~m}, 27 \mathrm{H}), 0.72-0.52(\mathrm{~m}, 18 \mathrm{H}) ; \mathrm{H}^{1}-\mathrm{H}^{3}$ nOe observed $1 \% ;{ }^{13} \mathrm{C}\left\{{ }^{1} \mathrm{H}\right\}$ NMR $\left(100 \mathrm{MHz}, \mathrm{CDCl}_{3}\right) \delta 157.2,136.3,128.0,127.9,125.1,99.8,86.3,74.3,71.6,61.8,6.9,6.8$, 
6.7, 5.2, 4.8, 4.4; IR (neat) $v_{\max }$ : 2953-2876 (C-H, aliphatic), 1677, 1493, 1103, 1002, $724 \mathrm{~cm}^{-1}$; HRMS (ESI) $m / z$ : [M $+\mathrm{H}]^{+}$Calcd for $\mathrm{C}_{30} \mathrm{H}_{57} \mathrm{O}_{4} \mathrm{Si}_{3}$ 565.3559; found 565.3555.

(2b) 3,4,6-Tri-O-triethylsilyl-2,5-anhydro-1-(3-nitrophenyl)-1-deoxy-D-ribino-hex-1-enitol.

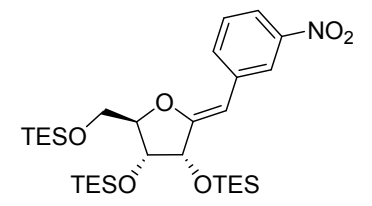

The title compound was prepared from exo-ribal 2 and 1-iodo-3-nitrobenzene following general procedure 2.1 for a duration of $24 \mathrm{~h}$. Purification by column chromatography using $2 \%$ ethyl acetate in hexanes afforded $2 \mathrm{~b}$ as a yellow oil $(77 \%, 37.0 \mathrm{mg}) .{ }^{1} \mathrm{H}$ NMR $\left(400 \mathrm{MHz}, \mathrm{CDCl}_{3}\right) \delta 8.42$ $(\mathrm{t}, J=1.9 \mathrm{~Hz}, 1 \mathrm{H}), 7.92(\mathrm{ddd}, J=8.2,2.3,0.9 \mathrm{~Hz}, 1 \mathrm{H}), 7.83(\mathrm{t}, J=7.9,1 \mathrm{H}), 7.38(\mathrm{t}, J=8.0 \mathrm{~Hz}$, $1 \mathrm{H}), 5.39(\mathrm{~s}, 1 \mathrm{H}), 4.66(\mathrm{dd}, J=4.6,0.8 \mathrm{~Hz}, 1 \mathrm{H}), 4.42-4.39(\mathrm{~m}, 1 \mathrm{H}), 4.23(\mathrm{t}, J=4.2 \mathrm{~Hz}, 1 \mathrm{H}), 3.94$ $(\mathrm{dd}, J=11.8,3.2 \mathrm{~Hz}, 1 \mathrm{H}), 3.81(\mathrm{dd}, J=11.8,2.8 \mathrm{~Hz}, 1 \mathrm{H}), 1.08-0.89(\mathrm{~m}, 27 \mathrm{H}), 0.74-0.58(\mathrm{~m}$, $18 \mathrm{H}) ; \mathrm{H}^{1}-\mathrm{H}^{3}$ nOe observed $1 \% ;{ }^{13} \mathrm{C}\left\{{ }^{1} \mathrm{H}\right\} \mathrm{NMR}\left(100 \mathrm{MHz}, \mathrm{CDCl}_{3}\right) \delta 160.2,148.4,138.2,133.5$, $128.7,122.2,119.6,97.1,87.6,74.1,71.8,61.8,6.9,6.8,6.6,5.1,4.9,4.3$; IR (neat) $v_{\max }: 2954-$ 2876 (C-H, aliphatic), 1677, 1528, 1458, 1104, 1002, $724 \mathrm{~cm}^{-1}$; HRMS (ESI) $\mathrm{m} / z:[\mathrm{M}+\mathrm{Na}]^{+} \mathrm{Calcd}$ for $\mathrm{C}_{30} \mathrm{H}_{55} \mathrm{NaO}_{6} \mathrm{Si}_{3}$ 632.3200; found 632.3200.

(2c) 3,4,6-Tri-O-triethylsilyl-2,5-anhydro-1-(4-cyanophenyl)-1-deoxy-D-ribino-hex-1-enitol.

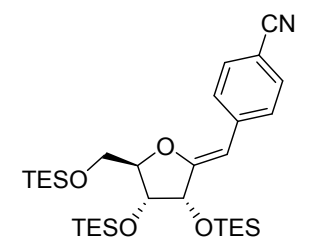

The title compound was prepared from exo-ribal 2 and 4-iodobenzonitrile following general procedure 2.1 for a duration of $24 \mathrm{~h}$. Purification by column chromatography using $2 \%$ ethyl acetate in hexanes afforded $2 \mathrm{c}$ as a yellow oil $(87 \%, 40.5 \mathrm{mg}) .{ }^{1} \mathrm{H}$ NMR $\left(400 \mathrm{MHz}, \mathrm{CDCl}_{3}\right) \delta 7.62$ $(\mathrm{d}, J=8.5 \mathrm{~Hz}, 2 \mathrm{H}), 7.51(\mathrm{~d}, J=8.5 \mathrm{~Hz}, 2 \mathrm{H}), 5.35(\mathrm{~s}, 1 \mathrm{H}), 4.65(\mathrm{dd}, J=4.6,0.8 \mathrm{~Hz}, 1 \mathrm{H}), 4.40-4.38$ (m, $1 \mathrm{H}), 4.21(\mathrm{dd}, J=4.4,3.9 \mathrm{~Hz}, 1 \mathrm{H}), 3.91(\mathrm{dd}, J=11.7,3.3 \mathrm{~Hz}, 1 \mathrm{H}), 3.80(\mathrm{dd}, J=11.7,3.0 \mathrm{~Hz}$, $1 \mathrm{H}), 1.04-0.90(\mathrm{~m}, 27 \mathrm{H}), 0.73-0.55(\mathrm{~m}, 18 \mathrm{H}) ; \mathrm{H}^{1}-\mathrm{H}^{3}$ nOe observed $1 \% ;{ }^{13} \mathrm{C}\left\{{ }^{1} \mathrm{H}\right\} \mathrm{NMR}(100 \mathrm{MHz}$, $\left.\mathrm{CDCl}_{3}\right) \delta 161.1,141.3,131.8,128.0,119.8,107.6,97.8,88.0,74.2,71.8,61.8,6.9,6.8,6.6,5.1$, 4.9, 4.3; IR (neat) $v_{\max }$ : 2954-2876 (C-H, aliphatic), $2222(\mathrm{CN}), 1672,1603,1458,1064,1003$, $723 \mathrm{~cm}^{-1}$; HRMS (ESI) $\mathrm{m} / z$ : [M $\left.+\mathrm{H}\right]^{+}$Calcd for $\mathrm{C}_{31} \mathrm{H}_{56} \mathrm{O}_{4} \mathrm{Si}_{3} 590.3512$; found 590.3524 .

(2d) 3,4,6-Tri-O-triethylsilyl-2,5-anhydro-1-(4-(hydroxymethyl)phenyl)-1-deoxy-D-ribino-hex-1enitol.

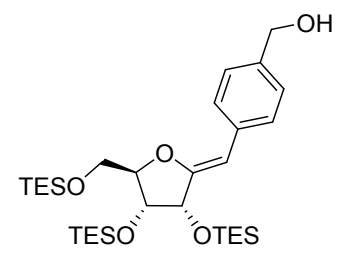


The title compound was prepared from exo-ribal 2 and (4-iodophenyl)methanol following general procedure 2.1 for a duration of $24 \mathrm{~h}$. Purification by column chromatography using $4 \%$ ethyl acetate in hexanes afforded $2 \mathrm{~d}$ as a colourless oil $(56 \%, 40.5 \mathrm{mg}) .{ }^{1} \mathrm{H} \mathrm{NMR}\left(400 \mathrm{MHz}, \mathrm{CDCl}_{3}\right) \delta$ $7.55(\mathrm{~d}, J=8.2 \mathrm{~Hz}, 2 \mathrm{H}), 7.25(\mathrm{~d}, J=8.2 \mathrm{~Hz}, 2 \mathrm{H}), 5.34(\mathrm{~s}, 1 \mathrm{H}), 4.63(\mathrm{~s}, 2 \mathrm{H}), 4.54(\mathrm{~d}, J=4.3 \mathrm{~Hz}$, $1 \mathrm{H}), 4.37-4.34(\mathrm{~m}, 1 \mathrm{H}), 4.17(\mathrm{dd}, J=5.0,4.6 \mathrm{~Hz}, 1 \mathrm{H}), 3.93(\mathrm{dd}, J=11.7,3.1 \mathrm{~Hz}, 1 \mathrm{H}), 3.78(\mathrm{dd}, J$ $=11.7,3.3 \mathrm{~Hz}, 1 \mathrm{H}), 1.59(\mathrm{bs}, 1 \mathrm{H}), 1.01-0.91(\mathrm{~m}, 27 \mathrm{H}), 0.71-0.56(\mathrm{~m}, 18 \mathrm{H}) ; \mathrm{H}^{1}-\mathrm{H}^{3}$ nOe observed $1 \% ;{ }^{13} \mathrm{C}\left\{{ }^{1} \mathrm{H}\right\} \mathrm{NMR}\left(100 \mathrm{MHz}, \mathrm{CDCl}_{3}\right) \delta 157.5,137.5,135.9,128.1,126.9,99.4,86.4,74.3,71.6$, $65.1,61.8,6.9,6.8,6.7,5.2,4.8,4.4$; IR (neat) $v_{\max }: 3359(\mathrm{OH}), 2953-2876(\mathrm{CH}$, aliphatic), 1678, 1458, 1159, 1102, 1002, $724 \mathrm{~cm}^{-1}$; HRMS (ESI) $\mathrm{m} / z$ : [M + Na] ${ }^{+}$Calcd for $\mathrm{C}_{31} \mathrm{H}_{59} \mathrm{O}_{5} \mathrm{Si}_{3} 595.3665$; found 595.3669 .

(2e) 3,4,6-Tri-O-triethylsilyl-2,5-anhydro-1-(4-formylphenyl)-1-deoxy-D-ribino-hex-1-enitol.

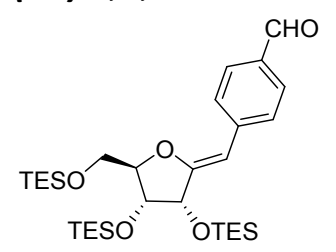

The title compound was prepared from exo-ribal 2 and 4-iodobenzaldehyde following general procedure 2.1 for a duration of $24 \mathrm{~h}$. Purification by column chromatography using $2 \%$ ethyl acetate in hexanes afforded $2 \mathrm{e}$ as a colourless oil $(73 \%, 34.1 \mathrm{mg}) .{ }^{1} \mathrm{H} \mathrm{NMR}\left(400 \mathrm{MHz}, \mathrm{CDCl}_{3}\right) \delta$ $9.92(\mathrm{~s}, 1 \mathrm{H}), 7.76(\mathrm{~d}, J=8.4 \mathrm{~Hz}, 2 \mathrm{H}), 7.69(\mathrm{~d}, J=8.4 \mathrm{~Hz}, 2 \mathrm{H}), 5.41(\mathrm{~s}, 1 \mathrm{H}), 4.65(\mathrm{dd}, J=4.5,0.7$ $\mathrm{Hz}, 1 \mathrm{H}), 4.42-4.40$ (m, 1H), 4.22 (dd, $J=4.4,4.2 \mathrm{~Hz}, 1 \mathrm{H}), 3.93$ (dd, $J=11.7,3.2 \mathrm{~Hz}, 1 \mathrm{H}), 3.81$ (dd, $J=11.7,3.0 \mathrm{~Hz}, 1 \mathrm{H}), 1.03-0.91(\mathrm{~m}, 27 \mathrm{H}), 0.73-0.56(\mathrm{~m}, 18 \mathrm{H}) ; \mathrm{H}^{1}-\mathrm{H}^{3}$ nOe observed $1 \%$; ${ }^{13} \mathrm{C}\left\{{ }^{1} \mathrm{H}\right\}$ NMR $\left(100 \mathrm{MHz}, \mathrm{CDCl}_{3}\right) \delta 191.8,161.0,143.1,133.0,129.8,128.0,98.5,87.9,74.3$, $71.8,61.8,6.9,6.8,6.7,6.7,5.2,4.9,4.3$; IR (neat) $v_{\max }: 2953-2876(\mathrm{C}-\mathrm{H}$, aliphatic), 1697 (C=O, aldehyde), 1671, 1599, 1161, 1101, 1003, $723 \mathrm{~cm}^{-1}$; HRMS (ESI) $\mathrm{m} / z:[\mathrm{M}+\mathrm{H}]^{+}$Calcd for $\mathrm{C}_{31} \mathrm{H}_{57} \mathrm{O}_{5} \mathrm{Si}_{3}$ 593.3500; found 593.3500.

(2f) 3,4,6-Tri-O-triethylsilyl-2,5-anhydro-1-((4-methoxycarbonyl)phenyl)-1-deoxy-D-ribino-hex-1enitol.

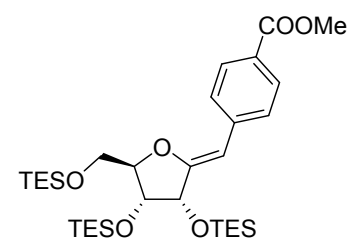

The title compound was prepared from exo-ribal 2 and methyl 4-iodobenzoate following general procedure 2.1 for a duration of $24 \mathrm{~h}$. Purification by column chromatography using $2 \%$ ethyl acetate in hexanes afforded $2 \mathrm{f}$ as a colourless oil $(60 \%, 30.0 \mathrm{mg}) .{ }^{1} \mathrm{H}$ NMR $\left(400 \mathrm{MHz}, \mathrm{CDCl}_{3}\right) \delta$ $9.92(\mathrm{~s}, 1 \mathrm{H}), 7.77-7.75(\mathrm{~m}, 2 \mathrm{H}), 7.70-7.68(\mathrm{~m}, 2 \mathrm{H}), 5.41(\mathrm{~s}, 1 \mathrm{H}), 4.65(\mathrm{dd}, J=4.5,0.7 \mathrm{~Hz}, 1 \mathrm{H})$, 4.41 (dd, $J=7.0,3.2 \mathrm{~Hz}, 1 \mathrm{H}), 4.22(\mathrm{dd}, J=4.4,4.1 \mathrm{~Hz}, 1 \mathrm{H}), 3.93(\mathrm{dd}, J=11.7,3.2 \mathrm{~Hz}, 1 \mathrm{H}), 3.81$ (dd, $J=11.7,3.0 \mathrm{~Hz}, 1 \mathrm{H}), 1.03-0.90(\mathrm{~m}, 27 \mathrm{H}), 0.73-0.56(\mathrm{~m}, 18 \mathrm{H}) ; \mathrm{H}^{1}-\mathrm{H}^{3}$ nOe observed $2 \%$; ${ }^{13} \mathrm{C}\left\{{ }^{1} \mathrm{H}\right\}$ NMR $\left(100 \mathrm{MHz}, \mathrm{CDCl}_{3}\right) \delta 191.8,161.0,143.1,133.0,129.8,128.0,98.5,87.9,74.3$, 
71.8, 61.8, 6.9, 6.8, 6.7, 6.7, 5.2, 4.9, 4.3; IR (neat) $v_{\max }$ : 2953-2876 (C-H, aliphatic), $1720(\mathrm{C}=\mathrm{O}$, ester), 1673, 1605, 1275, 1105, $724 \mathrm{~cm}^{-1}$; HRMS (ESI) $m / z$ : $[\mathrm{M}+\mathrm{Na}]^{+}$Calcd for $\mathrm{C}_{32} \mathrm{H}_{58} \mathrm{NaO}_{6} \mathrm{Si}_{3}$ 645.3433; found 645.3433.

(2g) 3,4,6-Tri-O-triethylsilyl-2,5-anhydro-1-(4-chlorophenyl)-1-deoxy-D-ribino-hex-1-enitol.

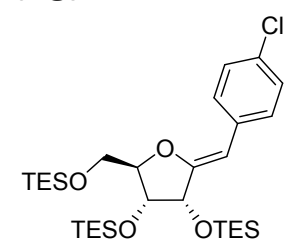

The title compound was prepared from exo-ribal 2 and 1-chloro-4-iodobenzene following general procedure 2.1 for a duration of $24 \mathrm{~h}$. Purification by column chromatography using $2 \%$ ethyl acetate in hexanes afforded $2 \mathrm{~g}$ as a colourless oil $(58 \%, 27.3 \mathrm{mg}) .{ }^{1} \mathrm{H}$ NMR $\left(400 \mathrm{MHz}, \mathrm{CDCl}_{3}\right) \delta$ $7.48(\mathrm{~d}, J=8.6 \mathrm{~Hz}, 2 \mathrm{H}), 7.20(\mathrm{~d}, J=8.6 \mathrm{~Hz}, 2 \mathrm{H}), 5.29(\mathrm{~s}, 1 \mathrm{H}), 4.55(\mathrm{~d}, J=4.2 \mathrm{~Hz}, 1 \mathrm{H}), 4.26-4.24$ $(\mathrm{m}, 1 \mathrm{H}), 4.19(\mathrm{t}, J=4.7 \mathrm{~Hz}, 1 \mathrm{H}), 3.91(\mathrm{dd}, J=11.7,3.1 \mathrm{~Hz}, 1 \mathrm{H}), 3.78(\mathrm{dd}, J=11.7,3.2 \mathrm{~Hz}, 1 \mathrm{H})$, 1.01-0.90 (m, 27H), 0.71-0.55 (m, 18H); $\mathrm{H}^{1}-\mathrm{H}^{3}$ nOe observed $2 \% ;{ }^{13} \mathrm{C}\left\{{ }^{1} \mathrm{H}\right\}$ NMR $(100 \mathrm{MHz}$, $\left.\mathrm{CDCl}_{3}\right) \delta 157.8,134.8,130.3,129.1,128.0,98.5,86.7,74.2,71.6,61.7,6.9,6.8,6.7,5.2,4.8,4.4$; IR (neat) $v_{\max }: 2953-2876\left(\mathrm{C}-\mathrm{H}\right.$, aliphatic), 1676, 1450, $724 \mathrm{~cm}^{-1}$; HRMS (ESI) $m / z:[\mathrm{M}+\mathrm{H}]^{+}$ Calcd for $\mathrm{C}_{30} \mathrm{H}_{56} \mathrm{ClO}_{4} \mathrm{Si}_{3}$ 599.3169; found 599.3168.

(2h) 3,4,6-Tri-O-triethylsilyl-2,5-anhydro-1-(4.bromophenyl)-1-deoxy-D-ribino-hex-1-enitol.

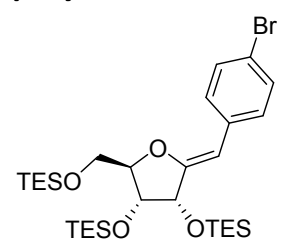

The title compound was prepared from exo-ribal 2 and 1-bromo-4-iodobenzene following general procedure 2.1 for a duration of $24 \mathrm{~h}$. Purification by column chromatography using $2 \%$ ethyl acetate in hexanes afforded $2 \mathrm{~h}$ as a colourless oil $(55 \%, 28.0 \mathrm{mg}) .{ }^{1} \mathrm{H} \mathrm{NMR}\left(400 \mathrm{MHz}, \mathrm{CDCl}_{3}\right) \delta$ $7.42(\mathrm{~d}, J=8.6 \mathrm{~Hz}, 2 \mathrm{H}), 7.35(\mathrm{~d}, J=8.6 \mathrm{~Hz}, 2 \mathrm{H}), 5.27(\mathrm{~s}, 1 \mathrm{H}), 4.55(\mathrm{~d}, J=4.1 \mathrm{~Hz}, 1 \mathrm{H}), 4.36-4.34$ (m, 1H), $4.18(\mathrm{t}, J=4.7 \mathrm{~Hz}, 1 \mathrm{H}), 3.91(\mathrm{dd}, J=11.7,3.1 \mathrm{~Hz}, 1 \mathrm{H}), 3.78$ (dd, $J=11.7,3.1 \mathrm{~Hz}, 1 \mathrm{H})$, 1.01-0.90 (m, 27H), 0.71-0.55 (m, 18H); $\mathrm{H}^{1}-\mathrm{H}^{3}$ nOe observed $2 \% ;{ }^{13} \mathrm{C}\left\{{ }^{1} \mathrm{H}\right\}$ NMR $(100 \mathrm{MHz}$, $\left.\mathrm{CDCl}_{3}\right) \delta 158.0,135.3,131.0,129.4,118.4,98.5,86.8,74.2,71.6,61.7,6.9,6.8,6.7,5.2,4.8,4.4$; IR (neat) $v_{\max }: 2953-2876\left(\mathrm{C}-\mathrm{H}\right.$, aliphatic), 1676, 1487, $723 \mathrm{~cm}^{-1}$; HRMS (ESI) $m / z:[\mathrm{M}+\mathrm{H}]^{+}$ Calcd for $\mathrm{C}_{30} \mathrm{H}_{56} \mathrm{BrO}_{4} \mathrm{Si}_{3}$ 643.2664; found 643.2661.

(3a) 3,4,6-Tri-O-triethylsilyl-2,5-anhydro-1-phenyl-1-deoxy-D-xylono-hex-1-enitol.

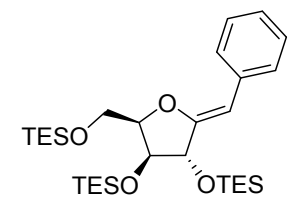


The title compound was prepared from exo-glycal 3 and iodobenzene following general procedure 2.1 for a duration of $24 \mathrm{~h}$. Purification by column chromatography using $2 \%$ ethyl acetate in hexanes afforded $3 \mathrm{a}$ as a colourless oil $(71 \%, 31.6 \mathrm{mg}) .{ }^{1} \mathrm{H}$ NMR $\left(400 \mathrm{MHz}, \mathrm{CDCl}_{3}\right) \delta 7.60(\mathrm{dd}$, $J=8.4,0.9 \mathrm{~Hz}, 2 \mathrm{H}), 7.27(\mathrm{t}, J=7.8 \mathrm{~Hz}, 2 \mathrm{H}), 7.11(\mathrm{tt}, J=7.4,1.2 \mathrm{~Hz}, 1 \mathrm{H}), 5.35(\mathrm{~s}, 1 \mathrm{H}), 4.60$ (td, $J=5.7,3.4 \mathrm{~Hz}, 1 \mathrm{H}), 4.36(\mathrm{~d}, J=1.9 \mathrm{~Hz}, 1 \mathrm{H}), 4.07(\mathrm{dd}, J=3.3,2.0 \mathrm{~Hz}, 1 \mathrm{H}), 3.92(\mathrm{dd}, J=5.7,2.4$ $\mathrm{Hz}, 2 \mathrm{H}), 3.78(\mathrm{dd}, J=11.7,3.3 \mathrm{~Hz}, 1 \mathrm{H}), 1.02-0.92(\mathrm{~m}, 27 \mathrm{H}), 0.68-0.60(\mathrm{~m}, 18 \mathrm{H}) ; \mathrm{H}^{1}-\mathrm{H}^{3} \mathrm{nOe}$ observed $2 \% ;{ }^{13} \mathrm{C}\left\{{ }^{1} \mathrm{H}\right\}$ NMR $\left(100 \mathrm{MHz}, \mathrm{CDCl}_{3}\right) \delta 157.5,136.5,128.0,127.9,125.2,100.9,85.5$, 79.5, 76.0, 61.0, 6.8, 5.1, 4.8, 4.4; IR (neat) $v_{\max }: 2954-2876$ (C-H, aliphatic), 1681, 1457, 1078, $1003,723 \mathrm{~cm}^{-1}$; HRMS (ESI) $m / z$ : [M+Na] $]^{+}$Calcd for $\mathrm{C}_{30} \mathrm{H}_{56} \mathrm{NaO}_{4} \mathrm{Si}_{3}$ 587.3379; found 587.3379.

(3b) 3,4,6-Tri-O-triethylsilyl-2,5-anhydro-1-(3-nitrophenyl)-1-deoxy-D-xylono-hex-1-enitol.

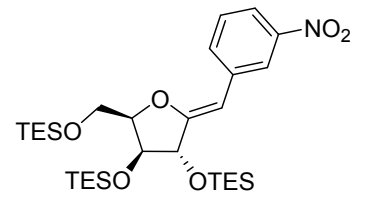

The title compound was prepared from exo-glycal 3 and 1-iodo-3-nitrobenzene following general procedure 2.1 for a duration of $24 \mathrm{~h}$. Purification by column chromatography using $4 \%$ ethyl acetate in hexanes afforded $3 \mathrm{~b}$ as a yellow oil $(53 \%, 25.5 \mathrm{mg}) .{ }^{1} \mathrm{H}$ NMR $\left(400 \mathrm{MHz}, \mathrm{CDCl}_{3}\right) \delta$ 8.37-8.36 (m, 1H), 7.95-7.92 (m, 2H), $7.40(\mathrm{t}, J=8.0 \mathrm{~Hz}, 1 \mathrm{H}), 5.40(\mathrm{~s}, 1 \mathrm{H}), 4.65(\mathrm{~m}, 1 \mathrm{H}), 4.42(\mathrm{~d}$, $J=2.2 \mathrm{~Hz}, 1 \mathrm{H}), 4.10(\mathrm{dd}, J=3.5,2.4 \mathrm{~Hz}, 1 \mathrm{H}), 3.93(\mathrm{~d}, J=5.6 \mathrm{~Hz}, 2 \mathrm{H}), 1.00-0.94(\mathrm{~m}, 27 \mathrm{H}), 0.70-$ $0.60(\mathrm{~m}, 18 \mathrm{H}) ; \mathrm{H}^{1}-\mathrm{H}^{3}$ nOe observed $2 \% ;{ }^{13} \mathrm{C}\left\{{ }^{1} \mathrm{H}\right\} \mathrm{NMR}\left(100 \mathrm{MHz}, \mathrm{CDCl}_{3}\right) \delta 160.2,148.4,138.2$, 133.6, 128.8, 122.3, 119.7, 98.6, 85.9, 79.4, 75.9, 60.7, 6.8, 5.0, 4.8, 4.4; IR (neat) $v_{\max }$ : 2954-2876 (C-H, aliphatic), 1675, 1529, 1348, 1089, 1003, $724 \mathrm{~cm}^{-1}$; HRMS (ESI) $\mathrm{m} / \mathrm{z}:[\mathrm{M}+\mathrm{H}]^{+} \mathrm{Calcd}$ for $\mathrm{C}_{30} \mathrm{H}_{56} \mathrm{NO}_{6} \mathrm{Si}_{3}$ 610.3410; found 610.3412.

(3c) 3,4,6-Tri-O-triethylsilyl-2,5-anhydro-1-(4-cyanophenyl)-1-deoxy-D-xylono-hex-1-enitol.

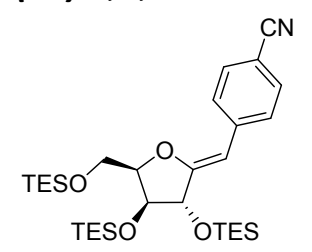

The title compound was prepared from exo-glycal 3 and 4-iodobenzonitrile following general procedure 2.1 for a duration of $24 \mathrm{~h}$. Purification by column chromatography using $4 \%$ ethyl acetate in hexanes afforded $3 \mathrm{c}$ as a colourless oil $(65 \%, 30.2 \mathrm{mg}) .{ }^{1} \mathrm{H}$ NMR $\left(400 \mathrm{MHz}, \mathrm{CDCl}_{3}\right) \delta$ 7.66-7.63 (m, 2H), 7.55-7.53 (m, 2H), $5.35(\mathrm{~s}, 1 \mathrm{H}), 4.64-4.61(\mathrm{~m}, 1 \mathrm{H}), 4.40(\mathrm{~d}, J=2.3 \mathrm{~Hz}, 1 \mathrm{H})$, 4.09 (dd, $J=3.5,2.3 \mathrm{~Hz}, 1 \mathrm{H}), 3.92(\mathrm{~d}, J=5.6 \mathrm{~Hz}, 2 \mathrm{H}), 1.00-0.94(\mathrm{~m}, 27 \mathrm{H}), 0.67-0.60(\mathrm{~m}, 18 \mathrm{H})$; $\mathrm{H}^{1}-\mathrm{H}^{3}$ nOe observed $2 \% ;{ }^{13} \mathrm{C}\left\{{ }^{1} \mathrm{H}\right\}$ NMR $\left(100 \mathrm{MHz}, \mathrm{CDCl}_{3}\right) \delta 161.1,141.3,131.8,128.1,119.7$, 107.8, 99.4, 88.2, 79.5, 75.9, 60.9, 6.7, 5.0, 4.8, 4.4; IR (neat) $v_{\max }: 2954-2876$ (C-H, aliphatic), $2223(\mathrm{CN}), 1670,1603,1458,1003,723 \mathrm{~cm}^{-1}$; HRMS (ESI) $\mathrm{m} / z$ : $[\mathrm{M}+\mathrm{Na}]^{+}$Calcd for $\mathrm{C}_{31} \mathrm{H}_{55} \mathrm{NNaO}_{4} \mathrm{Si}_{3} 612.3331$; found 612.3329 . 
(3d) 3,4,6-Tri-O-triethylsilyl-2,5-anhydro-1-(4-(hydroxymethyl)-phenyl)-1-deoxy-D-xylono-hex-1enitol.

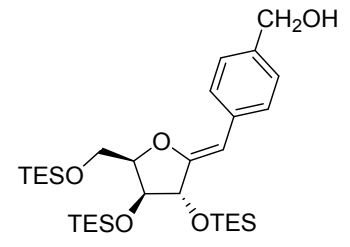

The title compound was prepared from exo-glycal 3 and 4-iodobenzyl alcohol following general procedure 2.1 for a duration of $24 \mathrm{~h}$. Purification by column chromatography using $10 \%$ ethyl acetate in hexanes afforded $3 \mathrm{~d}$ as a colourless oil $(56 \%, 26.3 \mathrm{mg}) .{ }^{1} \mathrm{H}$ NMR $\left(400 \mathrm{MHz}, \mathrm{CDCl}_{3}\right) \delta$ 7.60-7.58 (m, 2H), 7.28-7.26 (m, 2H), $5.35(\mathrm{~s}, 1 \mathrm{H}), 4.63(\mathrm{~s}, 2 \mathrm{H}), 4.62-4.59(\mathrm{~m}, 1 \mathrm{H}), 4.37(\mathrm{~d}, J=$ $2.0 \mathrm{~Hz}, 1 \mathrm{H}), 4.07(\mathrm{dd}, J=3.3,2.0 \mathrm{~Hz}, 1 \mathrm{H}), 3.93-3.92(\mathrm{~m}, 2 \mathrm{H}), 1.01-0.91(\mathrm{~m}, 27 \mathrm{H}), 0.69-0.59(\mathrm{~m}$, $18 \mathrm{H}) ; \mathrm{H}^{1}-\mathrm{H}^{3}$ nOe observed $2 \% ;{ }^{13} \mathrm{C}\left\{{ }^{1} \mathrm{H}\right\}$ NMR $\left(100 \mathrm{MHz}, \mathrm{CDCl}_{3}\right) \delta 157.7,137.7,136.0,128.1$, $126.9,100.5,85.5,79.5,76.0,65.4,61.0,6.8,6.7,6.7,5.1,4.8,4.4$; IR (neat) $v_{\max }: 3368(\mathrm{OH})$, 2953-2876 (C-H, aliphatic), 1678, 1458, 1003, $723 \mathrm{~cm}^{-1}$; HRMS (ESI) $\mathrm{m} / z$ : $[\mathrm{M}+\mathrm{H}]^{+}$Calcd for $\mathrm{C}_{31} \mathrm{H}_{59} \mathrm{O}_{5} \mathrm{Si}_{3}$ 595.3665; found 595.3672.

(3e) 3,4,6-Tri-O-triethylsilyl-2,5-anhydro-1-(4-formylphenyl)-1-deoxy-D-xylono-hex-1-enitol.

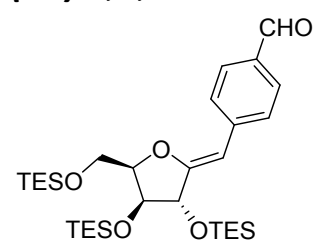

The title compound was prepared from exo-glycal 3 and 4-iodobenzaldehyde following general procedure 2.1 for a duration of $24 \mathrm{~h}$. Purification by column chromatography using $10 \%$ ethyl acetate in hexanes afforded $3 \mathrm{e}$ as a colourless oil $(70 \%, 32.7 \mathrm{mg}) .{ }^{1} \mathrm{H}$ NMR $\left(400 \mathrm{MHz}, \mathrm{CDCl}_{3}\right) \delta$ $9.92(\mathrm{~s}, 1 \mathrm{H}), 7.78-7.76(\mathrm{~m}, 2 \mathrm{H}), 7.73-7.71(\mathrm{~m}, 2 \mathrm{H}), 5.41(\mathrm{~s}, 1 \mathrm{H}), 4.64(\mathrm{td}, J=8.3,3.6 \mathrm{~Hz}, 1 \mathrm{H})$, $4.42(\mathrm{~d}, J=2.2 \mathrm{~Hz}, 1 \mathrm{H}), 4.10(\mathrm{dd}, J=3.5,2.3 \mathrm{~Hz}, 1 \mathrm{H}), 3.93(\mathrm{~d}, J=5.6,2 \mathrm{H}), 1.01-0.95(\mathrm{~m}, 27 \mathrm{H})$, 0.69-0.60 (m, 18H); $\mathrm{H}^{1}-\mathrm{H}^{3}$ nOe observed $2 \% ;{ }^{13} \mathrm{C}\left\{{ }^{1} \mathrm{H}\right\}$ NMR $\left(100 \mathrm{MHz}, \mathrm{CDCl}_{3}\right) \delta 191.8,161.0$, $143.1,133.2,129.8,128.1,99.9,86.2,79.6,75.9,60.9,6.7,5.0,4.8,4.4$; IR (neat) $v_{\max }: 2954-2876$ (C-H, aliphatic), 1698 (C=O, aldehyde), 1670, 1599, 1088, 1003, $724 \mathrm{~cm}^{-1}$; HRMS (ESI) $\mathrm{m} / z$ : [M $+\mathrm{H}]^{+}$Calcd for $\mathrm{C}_{31} \mathrm{H}_{57} \mathrm{O}_{5} \mathrm{Si}_{3}$ 595.3508; found 593.3505.

(3f) 3,4,6-Tri-O-triethylsilyl-2,5-anhydro-1-(4-(methoxycarbonyl)phenyl)-1-deoxy-D-xylono-hex1-enitol.

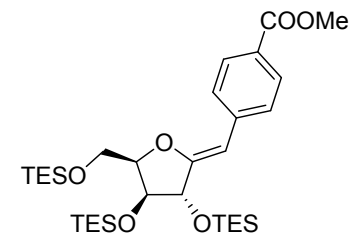

The title compound was prepared from exo-glycal 3 and methyl 4-iodobenzoate following general procedure 2.1 for a duration of $24 \mathrm{~h}$. Purification by column chromatography using $20 \%$ ethyl 
acetate in hexanes afforded $3 \mathrm{f}$ as a colourless oil $(65 \%, 32.0 \mathrm{mg}) .{ }^{1} \mathrm{H} \mathrm{NMR}\left(400 \mathrm{MHz}, \mathrm{CDCl}_{3}\right) \delta$ 7.95-7.92 (m, 2H), 7.65-7.62 (m, 2H), $5.39(\mathrm{~s}, 1 \mathrm{H}), 4.62(\mathrm{td}, J=8.3,3.5 \mathrm{~Hz}, 1 \mathrm{H}), 4.40(\mathrm{~d}, J=2.2$ $\mathrm{Hz}, 1 \mathrm{H}), 4.09$ (dd, $J=3.5,2.2 \mathrm{~Hz}, 1 \mathrm{H}), 3.93$ (d, $J=5.6,2 \mathrm{H}), 1.01-0.95(\mathrm{~m}, 27 \mathrm{H}), 0.69-0.60$ (m, $18 \mathrm{H}) ; \mathrm{H}^{1}-\mathrm{H}^{3}$ nOe observed $2 \% ;{ }^{13} \mathrm{C}\left\{{ }^{1} \mathrm{H}\right\}$ NMR $\left(100 \mathrm{MHz}, \mathrm{CDCl}_{3}\right) \delta 167.2,160.0,141.3,129.4$, $127.5,126.4,100.0,85.9,79.5,76.0,61.0,51.8,6.7,5.0,4.8,4.4$; IR (neat) $v_{\max }: 2953-2877$ (C$\mathrm{H}$, aliphatic), $1720\left(\mathrm{C}=\mathrm{O}\right.$, ester), 1672, 1606, 1489, 1273, $724 \mathrm{~cm}^{-1}$; HRMS (ESI) $\mathrm{m} / \mathrm{z}:[\mathrm{M}+\mathrm{H}]^{+}$ Calcd for $\mathrm{C}_{32} \mathrm{H}_{58} \mathrm{O}_{6} \mathrm{Si}_{3}$ 645.3433; found 645.3412.

(4a) 3,4,6-Tri-O-triethylsilyl-2,5-anhydro-1-phenyl-1-deoxy-D-arabino-hex-1-enitol.

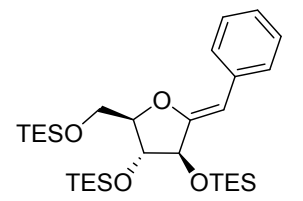

The title compound was prepared from exo-glycal 4 and iodobenzene following general procedure 2.1 for a duration of $24 \mathrm{~h}$. Purification by column chromatography using $2 \%$ ethyl acetate in hexanes afforded $6 \mathrm{a}$ as a colourless oil $(73 \%, 32.5 \mathrm{mg}) .{ }^{1} \mathrm{H}$ NMR $\left(400 \mathrm{MHz}, \mathrm{CDCl}_{3}\right) \delta 7.61(\mathrm{dd}$, $J=8.4,1.3 \mathrm{~Hz}, 2 \mathrm{H}), 7.28(\mathrm{t}, J=7.8 \mathrm{~Hz}, 2 \mathrm{H}), 7.12(\mathrm{tt}, J=7.4,1.2 \mathrm{~Hz}, 1 \mathrm{H}), 5.38(\mathrm{~s}, 1 \mathrm{H}), 4.46$ (ddd, $J=12.8,5.7,2.2 \mathrm{~Hz}, 1 \mathrm{H}), 4.17(\mathrm{dd}, J=2.4,2.4 \mathrm{~Hz}, 1 \mathrm{H}), 3.92-3.84(\mathrm{~m}, 2 \mathrm{H}), 1.03-0.97(\mathrm{~m}, 27 \mathrm{H})$, 0.73-0.61 (m, 18H); $\mathrm{H}^{1}-\mathrm{H}^{3}$ nOe observed $1 \% ;{ }^{13} \mathrm{C}\left\{{ }^{1} \mathrm{H}\right\} \mathrm{NMR}\left(100 \mathrm{MHz}, \mathrm{CDCl}_{3}\right) \delta 157.9,136.4$, $128.0,127.9,125.2,100.8,89.4,80.0,76.5,62.3,6.8,6.8,6.7,5.0,4.8,4.4$; IR (neat) $v_{\max }: 2954-$ 2876 (C-H, aliphatic), 1682, 1457, 1080, 1002, $724 \mathrm{~cm}^{-1}$; HRMS (ESI) $\mathrm{m} / z$ : $[\mathrm{M}+\mathrm{H}]^{+}$Calcd for $\mathrm{C}_{30} \mathrm{H}_{57} \mathrm{O}_{4} \mathrm{Si}_{3} 565.3559$; found 565.3565 .

(4b) 3,4,6-Tri-O-triethylsilyl-2,5-anhydro-1-(3-nitrophenyl)-1-deoxy-D-arabino-hex-1-enitol.

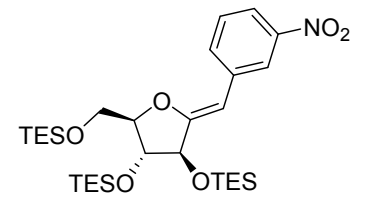

The title compound was prepared from exo-glycal 4 and 1-iodo-3-nitrobenzene following general procedure 2.1 for a duration of $24 \mathrm{~h}$. Purification by column chromatography using $4 \%$ ethyl acetate in hexanes afforded $4 \mathrm{~b}$ as a yellow oil $(47 \%, 22.6 \mathrm{mg}) .{ }^{1} \mathrm{H}$ NMR $\left(400 \mathrm{MHz}, \mathrm{CDCl}_{3}\right) \delta 8.41$ $(\mathrm{dd}, J=1.9,1.9 \mathrm{~Hz}, 1 \mathrm{H}), 7.94$ (ddd, $J=8.2,2.2,0.8 \mathrm{~Hz}, 1 \mathrm{H}), 7.87$ (d, $J=7.9 \mathrm{~Hz}, 1 \mathrm{H}), 7.40(\mathrm{t}, J$ $=8.0 \mathrm{~Hz}, 1 \mathrm{H}), 5.40(\mathrm{~s}, 1 \mathrm{H}), 4.47(\mathrm{~d}, J=2.9 \mathrm{~Hz}, 1 \mathrm{H}), 4.37-4.32(\mathrm{~m}, 1 \mathrm{H}), 4.18(\mathrm{t}, J=2.8 \mathrm{~Hz}, 1 \mathrm{H})$, 3.92-3.84 (m, 2H), 1.02-0.93 (m, 27H), 0.72-0.58 (m, 18H); $\mathrm{H}^{1}-\mathrm{H}^{3}$ nOe observed $2 \% ;{ }^{3} \mathrm{C}\left\{{ }^{1} \mathrm{H}\right\}$ NMR $\left(100 \mathrm{MHz}, \mathrm{CDCl}_{3}\right) \delta 160.5,148.4,138.1,133.5,128.7,122.3,119.8,98.6,89.7,79.9,76.1$, 61.9, 6.8, 6.8, 6.7, 5.0, 4.8, 4.7, 4.3; IR (neat) $v_{\max }: 2954-2876$ (C-H, aliphatic), 1675, 1530, 1349, 1085, 1003, $725 \mathrm{~cm}^{-1}$; HRMS (ESI) $m / z$ : $[\mathrm{M}+\mathrm{H}]^{+}$Calcd for $\mathrm{C}_{30} \mathrm{H}_{56} \mathrm{NO}_{6} \mathrm{Si}_{3}$ 610.3410; found 610.3434 . 
(4c) 3,4,6-Tri-O-triethylsilyl-2,5-anhydro-1-(4-cyanophenyl)-1-deoxy-D-arabino-hex-1-enitol.

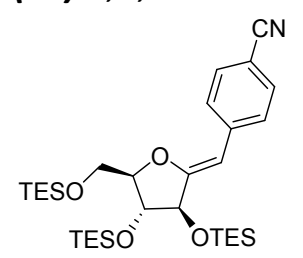

The title compound was prepared from exo-glycal 4 and 4-iodobenzonitrile following general procedure 2.1 for a duration of $24 \mathrm{~h}$. Purification by column chromatography using $4 \%$ ethyl acetate in hexanes afforded $4 \mathrm{c}$ as a colourless oil $(58 \%, 27.0 \mathrm{mg}) .{ }^{1} \mathrm{H} \mathrm{NMR}\left(400 \mathrm{MHz}, \mathrm{CDCl}_{3}\right) \delta$ 7.64-7.62 (m, 2H), 7.53-7.51 (m, 2H), $5.36(\mathrm{~s}, 1 \mathrm{H}), 4.46(\mathrm{~d}, J=2.9 \mathrm{~Hz}, 1 \mathrm{H}), 4.35-4.32(\mathrm{~m}, 1 \mathrm{H})$, $4.16(\mathrm{t}, J=2.8 \mathrm{~Hz}, 1 \mathrm{H}), 3.89-3.82(\mathrm{~m}, 2 \mathrm{H}), 1.01-0.94(\mathrm{~m}, 27 \mathrm{H}), 0.71-0.58(\mathrm{~m}, 18 \mathrm{H}) ; \mathrm{H}^{1}-\mathrm{H}^{3} \mathrm{nOe}$ observed $1 \% ;{ }^{13} \mathrm{C}\left\{{ }^{1} \mathrm{H}\right\}$ NMR $\left(100 \mathrm{MHz}, \mathrm{CDCl}_{3}\right) \delta 161.3,141.2,131.8,128.0,119.7,107.8,99.3$, 88.8, 80.0, 76.2, 61.9, 6.8, 6.7, 6.7, 5.0, 4.7, 4.3; IR (neat) $v_{\max }$ : 2954-2876 (C-H, aliphatic), 2223 (CN), 1669, 1603, 1002, $724 \mathrm{~cm}^{-1}$; HRMS (ESI) $m / z$ : $[\mathrm{M}+\mathrm{Na}]^{+}$Calcd for $\mathrm{C}_{31} \mathrm{H}_{55} \mathrm{NNaO}_{4} \mathrm{Si}_{3}$ 612.3300; found 612.3300 .

(4d) 3,4,6-Tri-O-triethylsilyl-2,5-anhydro-1-(4-(hydroxymethyl)-phenyl)-1-deoxy-D-arabino-hex1-enitol.

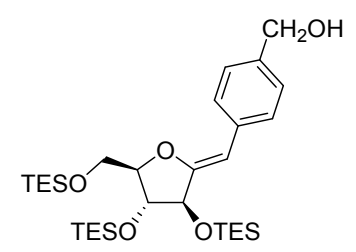

The title compound was prepared from exo-glycal 4 and 4-iodobenzyl alcohol following general procedure 2.1 for a duration of $24 \mathrm{~h}$. Purification by column chromatography using $10 \%$ ethyl acetate in hexanes afforded $4 \mathrm{~d}$ as a colourless oil $(53 \%, 24.9 \mathrm{mg}) .{ }^{1} \mathrm{H}$ NMR $\left(400 \mathrm{MHz}, \mathrm{CDCl}_{3}\right) \delta$ 7.59-7.57 (m, 2H), 7.28-7.26 (m, 2H), 5.37 (s, 1H), $4.63(\mathrm{~s}, 2 \mathrm{H}), 4.44$ (d, $J=2.5 \mathrm{~Hz}, 1 \mathrm{H}), 4.33-$ $4.30(\mathrm{~m}, 1 \mathrm{H}), 4.15(\mathrm{t}, J=4.8 \mathrm{~Hz}, 1 \mathrm{H}), 3.90-3.83(\mathrm{~m}, 2 \mathrm{H}), 1.01-0.96(\mathrm{~m}, 27 \mathrm{H}), 0.71-0.60(\mathrm{~m}, 18 \mathrm{H})$; $\mathrm{H}^{1}-\mathrm{H}^{3}$ nOe observed $1 \% ;{ }^{13} \mathrm{C}\left\{{ }^{1} \mathrm{H}\right\}$ NMR $\left(100 \mathrm{MHz}, \mathrm{CDCl}_{3}\right) \delta 158.0,137.7,135.9,128.0,126.9$, $100.5,89.4,70.0,76.5,65.4,62.3,6.8,6.7,6.7,5.0,4.8,4.4$; IR (neat) $v_{\max }: 3383(\mathrm{OH}), 2954-$ 2876 (C-H, aliphatic), 1677, 1458, 1102, 1002, $724 \mathrm{~cm}^{-1}$; HRMS (ESI) $m / z:[\mathrm{M}+\mathrm{H}]^{+}$Calcd for $\mathrm{C}_{31} \mathrm{H}_{59} \mathrm{O}_{5} \mathrm{Si}_{3}$ 595.3665; found 595.3666.

(4e) 3,4,6-Tri-O-triethylsilyl-2,5-anhydro-1-(4-formylphenyl)-1-deoxy-D-arabino-hex-1-enitol.

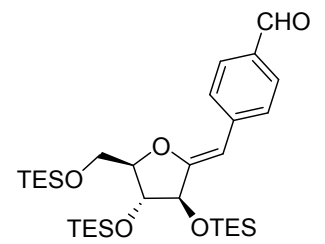

The title compound was prepared from exo-glycal 4 and 4-iodobenzaldehyde following general procedure 2.1 for a duration of $24 \mathrm{~h}$. Purification by column chromatography using $10 \%$ ethyl acetate in hexanes afforded $4 \mathrm{e}$ as a colourless oil $(61 \%, 28.5 \mathrm{mg}) .{ }^{1} \mathrm{H} \mathrm{NMR}\left(400 \mathrm{MHz}, \mathrm{CDCl}_{3}\right) \delta$ 
$9.92(\mathrm{~s}, 1 \mathrm{H}), 7.78-7.76(\mathrm{~m}, 2 \mathrm{H}), 7.71-7.70(\mathrm{~m}, 2 \mathrm{H}), 5.42(\mathrm{~s}, 1 \mathrm{H}), 4.48(\mathrm{~d}, J=2.9 \mathrm{~Hz}, 1 \mathrm{H}), 4.42$ (ddd, $J=6.5,5.7,2.6 \mathrm{~Hz}, 1 \mathrm{H}), 4.17(\mathrm{t}, J=2.8 \mathrm{~Hz}, 1 \mathrm{H}), 3.84(\mathrm{dd}, J=10.6,6.61 \mathrm{H}), 3.86(\mathrm{dd}, J=$ 10.6, $5.6 \mathrm{~Hz}), 1.04-0.92(\mathrm{~m}, 27 \mathrm{H}), 0.72-0.58(\mathrm{~m}, 18 \mathrm{H}) ; \mathrm{H}^{1}-\mathrm{H}^{3}$ nOe observed $2 \% ;{ }^{13} \mathrm{C}\left\{{ }^{1} \mathrm{H}\right\} \mathrm{NMR}$ $\left(100 \mathrm{MHz}, \mathrm{CDCl}_{3}\right) \delta 191.8,161.3,143.0,133.2,129.8,128.0,127.9,99.8,89.7,80.1,76.1,62.0$, 6.8, 6.7, 6.7, 5.0, 4.8, 4.3; IR (neat) $v_{\max }: 2954-2876$ (C-H, aliphatic), 1698 (C=O, aldehyde), 1670, $1599,1084,1002,724 \mathrm{~cm}^{-1}$; HRMS (ESI) $m / z$ : $[\mathrm{M}+\mathrm{H}]^{+}$Calcd for $\mathrm{C}_{31} \mathrm{H}_{57} \mathrm{O}_{5} \mathrm{Si}_{3}$ 595.3508; found 593.3507 .

(4f) 3,4,6-Tri-O-triethylsilyl-2,5-anhydro-1-(4-(methoxycarbonyl)phenyl)-1-deoxy-D-arabino-hex1-enitol.

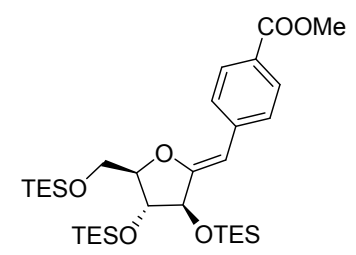

The title compound was prepared from exo-glycal 4 and methyl 4-iodobenzoate following general procedure 2.1 for a duration of $24 \mathrm{~h}$. Purification by column chromatography using $20 \%$ ethyl acetate in hexanes afforded $4 \mathrm{f}$ as a colourless oil $(73 \%, 35.9 \mathrm{mg}) .{ }^{1} \mathrm{H}$ NMR $\left(400 \mathrm{MHz}, \mathrm{CDCl}_{3}\right) \delta$ 7.93-7.92 (m, 2H), 7.62-7.60 (m, 2H), $5.39(\mathrm{~s}, 1 \mathrm{H}), 4.62(\mathrm{td}, J=8.3,3.5 \mathrm{~Hz}, 1 \mathrm{H}), 4.40(\mathrm{~d}, J=2.2$ $\mathrm{Hz}, 1 \mathrm{H}), 4.09$ (dd, $J=3.5,2.2 \mathrm{~Hz}, 1 \mathrm{H}), 3.93(\mathrm{~d}, J=5.6,2 \mathrm{H}), 1.01-0.95(\mathrm{~m}, 27 \mathrm{H}), 0.69-0.60(\mathrm{~m}$, $18 \mathrm{H}) ; \mathrm{H}^{1}-\mathrm{H}^{3}$ nOe observed $2 \% ;{ }^{13} \mathrm{C}\left\{{ }^{1} \mathrm{H}\right\}$ NMR $\left(100 \mathrm{MHz}, \mathrm{CDCl}_{3}\right) \delta 167.2,160.0,141.3,129.4$, $127.5,126.4,100.0,85.9,79.5,76.0,61.0,51.8,6.7,5.0,4.8,4.4$; IR (neat) $v_{\max }: 2953-2876$ (C$\mathrm{H}$, aliphatic), $1720\left(\mathrm{C}=\mathrm{O}\right.$, ester), 1672, 1605, 1273, 1003, $723 \mathrm{~cm}^{-1}$; HRMS (ESI) $\mathrm{m} / \mathrm{z}:[\mathrm{M}+\mathrm{H}]^{+}$ Calcd for $\mathrm{C}_{32} \mathrm{H}_{58} \mathrm{O}_{6} \mathrm{Si}_{3}$ 645.3433; found 645.3412.

(5a) 3,4,5,7-Tetra-O-benzyl-2,6-anhydro-1-phenyl-1-deoxy-D-gluco-hept-1-enitol.

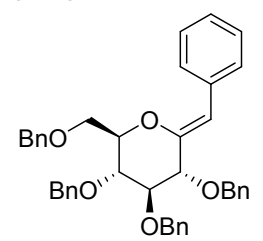

The title compound was prepared from exo-glucal 5 and iodobenzene following general procedure 2.1 for a duration of $24 \mathrm{~h}$. Purification by column chromatography using $10 \%$ ethyl acetate in hexanes afforded $5 \mathrm{a}$ as a colourless oil $(66 \%, 28.0 \mathrm{mg}) .{ }^{1} \mathrm{H}$ NMR $\left(400 \mathrm{MHz}, \mathrm{CDCl}_{3}\right) \delta 7.69(\mathrm{~d}, J$ $=7.2 \mathrm{~Hz}, 2 \mathrm{H}), 7.39-7.14(\mathrm{~m}, 23 \mathrm{H}), 5.72(\mathrm{~s}, 1 \mathrm{H}), 4.80-4.75(\mathrm{~m}, 3 \mathrm{H}), 4.68-4.55(\mathrm{~m}, 5 \mathrm{H}), 4.13-4.10$ $(\mathrm{m}, 1 \mathrm{H}), 4.04-4.03(\mathrm{~m}, 1 \mathrm{H}), 3.89-3.77(\mathrm{~m}, 4 \mathrm{H}) ; \mathrm{H}^{1}-\mathrm{H}^{3}$ nOe observed $2 \% ;{ }^{13} \mathrm{C}\left\{{ }^{1} \mathrm{H}\right\}$ NMR $(100$ $\left.\mathrm{MHz}, \mathrm{CDCl}_{3}\right) \delta 149.1,138.2,138.2,138.2,137.9,135.2,128.8,128.5,128.5,128.4,128.4,128.2$, 128.0, 127.9, 127.9, 127.9, 127.8, 127.8, 127.8, 127.7, 126.4, 109.5, 84.6, 79.3, 77.9, 76.9, 74.1, 73.5, 71.8, 69.2; IR (neat) $v_{\max }:$ 30287-3028 (C-H, aromatic), 2863 (C-H, aliphatic), 1668, 1494, $1452,1068,732,6.92 \mathrm{~cm}^{-1}$; HRMS (ESI) $m / z$ : $[\mathrm{M}+\mathrm{Na}]^{+} \mathrm{Calcd}$ for $\mathrm{C}_{41} \mathrm{H}_{40} \mathrm{NaO}_{5}$ 635.2800; found 635.2800 . 
(6a) 3,4,5,7-Tetra-O-benzyl-2,6-anhydro-1-phenyl-1-deoxy-D-galacto-hept-1-enitol.

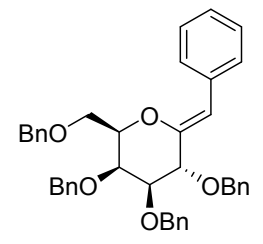

The title compound was prepared from exo-glycal 6 and iodobenzene following general procedure 2.1 for a duration of $24 \mathrm{~h}$. Purification by column chromatography using $10 \%$ ethyl acetate in hexanes afforded $6 \mathrm{a}$ as a colourless oil $(63 \%, 27 \mathrm{mg})$. Spectroscopic data is consistent with a previous report. ${ }^{12}{ }^{1} \mathrm{H}$ NMR $\left(400 \mathrm{MHz}, \mathrm{CDCl}_{3}\right) \delta 7.67(\mathrm{~d}, J=7.3 \mathrm{~Hz}, 2 \mathrm{H}), 7.40-7.12(\mathrm{~m}, 23 \mathrm{H})$, $5.96(\mathrm{~s}, 1 \mathrm{H}), 4.93(\mathrm{~d}, J=11.5 \mathrm{~Hz}, 1 \mathrm{H}), 4.80-4.71(\mathrm{~m}, 4 \mathrm{H}), 4.63(\mathrm{~d}, J=11.5 \mathrm{~Hz}, 1 \mathrm{H}), 4.47(\mathrm{~d}, J=$ $11.7 \mathrm{~Hz}, 1 \mathrm{H}), 4.43(\mathrm{~s}, 1 \mathrm{H}), 4.42(\mathrm{~d}, J=11.6 \mathrm{~Hz}, 1 \mathrm{H}), 4.12(\mathrm{t}, J=2.5 \mathrm{~Hz}, 1 \mathrm{H}), 4.05-4.01(\mathrm{~m}, 1 \mathrm{H})$, $3.84(\mathrm{dd}, J=9.9,6.8 \mathrm{~Hz}, 1 \mathrm{H}), 3.72(\mathrm{dd}, J=9.9,5.2 \mathrm{~Hz}, 1 \mathrm{H}) ; \mathrm{H}^{1}-\mathrm{H}^{3}$ nOe observed $2 \% ;{ }^{13} \mathrm{C}\left\{{ }^{1} \mathrm{H}\right\}$ NMR $\left(100 \mathrm{MHz}, \mathrm{CDCl}_{3}\right) \delta 150.1,138.44,138.38,138.1,138.0,135.1,128.9,128.4,128.38$, $128.35,128.3,128.1,128.0,127.9,127.7,127.7,127.6,127.6,127.5,126.4,110.8,81.5,78.3$, 77.4, 74.3, 74.0, 73.4, 73.3, 73.0, 69.3; IR (neat) $v_{\max }$ : 3086-3028 (CH, aromatic), $2865(\mathrm{CH}$, aliphatic), 1664, 1096, 731, $692 \mathrm{~cm}^{-1}$.

(7a) 3,4,5,7-Tetra-O-benzyl-2,6-anhydro-1-phenyl-1-deoxy-D-manno-hept-1-enitol.

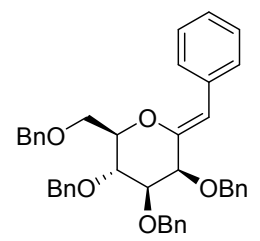

The title compound was prepared from exo-glycal 7 and iodobenzene following general procedure 2.1 for a duration of $24 \mathrm{~h}$. Purification by column chromatography using $10 \%$ ethyl acetate in hexanes afforded $7 \mathrm{a}$ as a colourless oil $(63 \%, 27 \mathrm{mg})$. Spectroscopic data is consistent with a previous report. ${ }^{12}{ }^{1} \mathrm{H}$ NMR $\left(400 \mathrm{MHz}, \mathrm{CDCl}_{3}\right) \delta 7.70(\mathrm{~d}, J=4.3 \mathrm{~Hz}, 2 \mathrm{H}), 7.43-7.16(\mathrm{~m}, 23 \mathrm{H})$, $5.58(\mathrm{~s}, 1 \mathrm{H}), 4.99(\mathrm{~d}, J=11.0 \mathrm{~Hz}, 1 \mathrm{H}), 4.80(\mathrm{~d}, J=12.7 \mathrm{~Hz}, 1 \mathrm{H}), 4.70(\mathrm{~d}, J=11.9 \mathrm{~Hz}, 1 \mathrm{H}), 4.66$ $(\mathrm{d}, J=11.9 \mathrm{~Hz}, 1 \mathrm{H}), 4.63-4.56(\mathrm{~m}, 3 \mathrm{H}), 4.48(\mathrm{~d}, J=12.7 \mathrm{~Hz}, 1 \mathrm{H}), 4.22(\mathrm{t}, J=9.3 \mathrm{~Hz}, 1 \mathrm{H}), 4.11$ $(\mathrm{d}, J=3.2 \mathrm{~Hz}, 1 \mathrm{H}), 3.93-3.86(\mathrm{~m}, 2 \mathrm{H}), 3.82-3.78(\mathrm{~m}, 1 \mathrm{H}), 3.74(\mathrm{dd}, J=9.1,3.2 \mathrm{~Hz}, 1 \mathrm{H}) ; \mathrm{H}^{1}-\mathrm{H}^{3}$ nOe observed $2 \% ;{ }^{13} \mathrm{C}\left\{{ }^{1} \mathrm{H}\right\}$ NMR $\left(100 \mathrm{MHz}, \mathrm{CDCl}_{3}\right) \delta 148.4,138.4,138.4,138.2,138.1,134.5$, $128.9,128.4,128.4,128.3,128.2,127.9,127.8,127.8,127.7,127.7,127.5,127.0,114.9,81.9$, $79.8,77.2,75.2,75.1,74.3,73.4,71.5,69.9,69.2$; IR (neat) $v_{\max }: 3062-3028$ (CH, aromatic), 29212885 (CH, aliphatic), 1661, 1453, 734, $694 \mathrm{~cm}^{-1}$.

(8a) 3,4,6-Tri-O-benzyl-2,5-anhydro-1-phenyl-1-deoxy-D-arabino-hex-1-enitol.

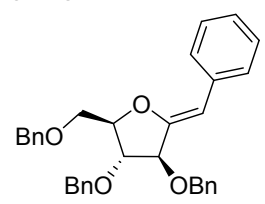


The title compound was prepared from exo-arabinal 8 and iodobenzene following general procedure 2.1 for a duration of $24 \mathrm{~h}$. Purification by column chromatography using $10 \%$ ethyl acetate in hexanes afforded $8 \mathrm{a}$ as a colourless oil $(51 \%, 20 \mathrm{mg}) .{ }^{1} \mathrm{H} \mathrm{NMR}\left(400 \mathrm{MHz}, \mathrm{CDCl}_{3}\right) \delta$ $7.60(\mathrm{~d}, J=7.6 \mathrm{~Hz}, 2 \mathrm{H}), 7.36-7.24(\mathrm{~m}, 17 \mathrm{H}), 7.13(\mathrm{t}, J=7.3 \mathrm{~Hz}, 1 \mathrm{H}), 5.46(\mathrm{~s}, 1 \mathrm{H}), 4.69-4.50(\mathrm{~m}$, $8 \mathrm{H}), 4.12(\mathrm{t}, J=2.5 \mathrm{~Hz}, 1 \mathrm{H}), 3.76-3.67(\mathrm{~m}, 2 \mathrm{H}) ; \mathrm{H}^{1}-\mathrm{H}^{3}$ nOe observed $1 \% ;{ }^{13} \mathrm{C}\left\{{ }^{1} \mathrm{H}\right\}$ NMR $(100$ $\left.\mathrm{MHz}, \mathrm{CDCl}_{3}\right) \delta 154.0,138.0,137.7,137.5,135.7,128.5,128.5,128.4,128.2,128.1,127.9,127.9$, $127.8,127.8,127.7,125.7,102.9,85.1,83.2,81.4,73.4,71.7,70.7,69.9$; IR (neat) $v_{\max }: 3087-$ 3028 (C-H, aromatic), 2923-2861 (C-H, aliphatic), 1675, 1452, 1070, 732, $692 \mathrm{~cm}^{-1}$; HRMS (ESI) $m / z:[\mathrm{M}+\mathrm{Na}]^{+}$Calcd for $\mathrm{C}_{33} \mathrm{H}_{32} \mathrm{NaO}_{4}$ 515.2194; found 515.2193.

(9a) 3,4,5,7-Tetra-O-acetyl-2,6-anhydro-1-phenyl-1-deoxy-D-glucono-hept-1-enitol.

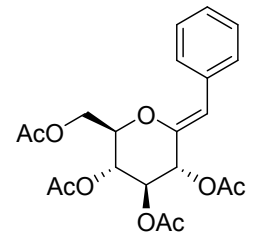

The title compound was prepared from acetylated exo-glucal 9 and iodobenzene following general procedure 2.1 for a duration of $24 \mathrm{~h}$. Purification by column chromatography using $30 \%$ ethyl acetate in hexanes afforded 9 a as a colourless oil $(72 \%, 25 \mathrm{mg})$. Spectroscopic data is consistent with a previous report. ${ }^{12}{ }^{1} \mathrm{H}$ NMR $\left(400 \mathrm{MHz}, \mathrm{CDCl}_{3}\right) \delta 7.62(\mathrm{~d}, J=7.3 \mathrm{~Hz}, 2 \mathrm{H}), 7.31(\mathrm{t}, J=11.1$ $\mathrm{Hz}, 2 \mathrm{H}), 7.23(\mathrm{tt}, J=11.0,1.1 \mathrm{~Hz}, 1 \mathrm{H}), 5.76(\mathrm{~s}, 1 \mathrm{H}), 5.55(\mathrm{dd}, J=5.7,1.0 \mathrm{~Hz}, 1 \mathrm{H}), 5.23-5.17(\mathrm{~m}$, 2H), 4.36 (dd, $J=12.4,2.6 \mathrm{~Hz}, 1 \mathrm{H}), 4.31$ (dd, $J=12.4,5.3 \mathrm{~Hz}, 1 \mathrm{H}), 4.11$ (ddd, $J=9.7,5.3,2.7$ $\mathrm{Hz}, 1 \mathrm{H}), 2.18(\mathrm{~s}, 3 \mathrm{H}), 2.13(\mathrm{~s}, 3 \mathrm{H}), 2.08(\mathrm{~s}, 3 \mathrm{H}), 2.07(\mathrm{~s}, 3 \mathrm{H}) ; \mathrm{H}^{1}-\mathrm{H}^{3}$ nOe observed $2 \% ;{ }^{13} \mathrm{C}\left\{{ }^{1} \mathrm{H}\right\}$ NMR $\left(100 \mathrm{MHz}, \mathrm{CDCl}_{3}\right) \delta 170.7,169.9,169.4,169.0,145.6,133.8,129.0,128.3,127.3,111.4$, 75.1, 73.4, 70.1, 68.7, 62.3, 20.9, 20.8, 20.7, 20.6; IR (neat) $v_{\max }: 1735$ (C=O, ester), 1216, 1033 $\mathrm{cm}^{-1}$.

(1x) 3,4,5,7-Tetra-O-triethylsilyl-2,6-anhydro-1-deoxy-1-phenyl-D-glycero-L-gulo-heptitol.
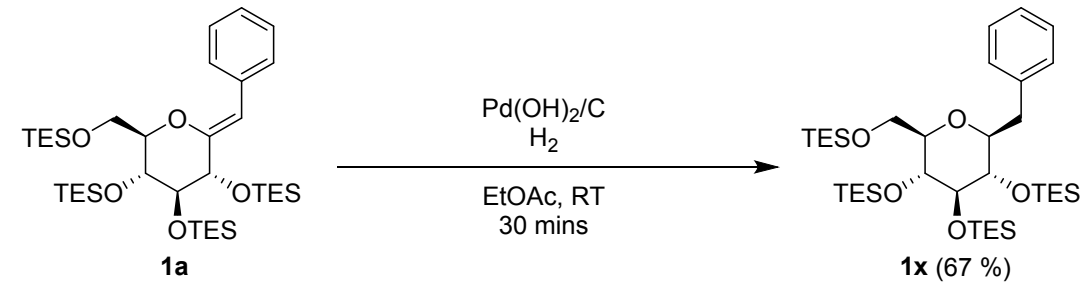

Catalytic hydrogenation was performed using a modified literature procedure. ${ }^{13} \mathrm{~A}$ suspension of $\mathrm{Pd} / \mathrm{C}(10 \mathrm{wt} . \%, 10 \mathrm{mg}, 9.1 \mu \mathrm{mol})$ was prepared in anhydrous and degassed EtOAc $(0.3 \mathrm{~mL})$ with vigorous stirring under a hydrogen atmosphere. A separate solution of aryl-exo-glucal (1a) (50

12 Griffin, Frank K.; Paterson, Duncan E.; Murphy, Paul V.; Taylor, Richard J. K. Eur. J. Org. Chem. 2002, 2002 (7), 1305-1322.

13. Kaszás, T.; Ivanov, A.; Tóth, M.; Ehlers, P.; Langer, P.; Somsák, L. Carbohydr. Res. 2018, $466,30-38$. 
$\mathrm{mg}, 0.07 \mathrm{mmol})$ was prepared in an oven-dried vile under argon using degassed EtOAc $(0.3 \mathrm{~mL})$, and was added to the $\mathrm{Pd} / \mathrm{C}$ at room temperature. The mixture was stirred for $30 \mathrm{mins}$ and then was filtered over celite. The solvent was removed and the product was purified by flash column chromatography using $0-5 \%$ EtOAc in hexanes to afford $\mathbf{1 x}$ as a pale-yellow oil (33.5 $\mathrm{mg}, 67$ \%). ${ }^{1} \mathrm{H}$ NMR (400 MHz, $\left.\mathrm{CDCl}_{3}\right) \delta$ 7.27-7.23 (m, 4H), 7.20-7.16 (m, 1H), 3.72-3.62 (m, 5H), 3.50 $(\mathrm{dd}, J=6.6,3.9 \mathrm{~Hz}, 1 \mathrm{H}), 3.41(\mathrm{dd}, J=10.2,5.1,1 \mathrm{H}), 3.00(\mathrm{dd}, J=14.2,3.1,1 \mathrm{H}), 2.70(\mathrm{dd}, J=$ 14.2, $9.3 \mathrm{~Hz}, 1 \mathrm{H}), 1.02-0.91(\mathrm{~m}, 36 \mathrm{H}), 0.71-0.54(\mathrm{~m}, 24 \mathrm{H}) ;{ }^{13} \mathrm{C}\left\{{ }^{1} \mathrm{H}\right\} \mathrm{NMR}\left(100 \mathrm{MHz}, \mathrm{CDCl}_{3}\right) \delta$ 139.8, 129.4, 128.0, 125.8, 81.2, 80.3, 78.7, 77.2, 75.8, 71.6, 63.7, 39.7, 7.1, 7.01, 7.0, 6.8, 5.4, 5.3, 5.1, 4.5; IR (neat) $v_{\max }: 2953-2876$ (C-H, aliphatic), 1457, 1090, 1003, $722 \mathrm{~cm}^{-1}$; HRMS (ESI) $m / z:[\mathrm{M}+\mathrm{H}]^{+}$Calcd for $\mathrm{C}_{37} \mathrm{H}_{75} \mathrm{O}_{5} \mathrm{Si}_{4} 711.4686$; found 711.4698 .

\subsection{Synthesis of 1-chloro-2-(4-ethoxybenzyl)-4-iodobenzene (10).}

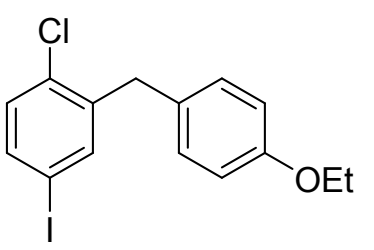

Aglycone 10 was prepared via a modified literature procedure. ${ }^{14}$ To a solution of 2-chloro-5iodobenzoic acid (1.0 g, $3.5 \mathrm{mmol})$ in anhydrous DCM $(5 \mathrm{~mL})$ and DMF $(4 \mu \mathrm{L}, 1.4 \mathrm{~mol} \%)$ under argon at room temperature was added a $2 \mathrm{M}$ solution of oxalyl chloride in DCM $(2.2 \mathrm{~mL}, 4.2$ $\mathrm{mmol})$. The reaction was stirred for $2 \mathrm{~h}$ and then was transferred to an anhydrous solution of ethoxybenzene $(500 \mu \mathrm{L}, 4.0 \mathrm{mmol})$ and $\mathrm{AlCl}_{3}(580 \mathrm{mg}, 4.34 \mathrm{mmol})$ in DCM under argon. Upon completion of the reaction $(\sim 3 \mathrm{~h}), 1 \mathrm{M} \mathrm{HCl}$ was added $(10 \mathrm{~mL})$ and the organic layer was

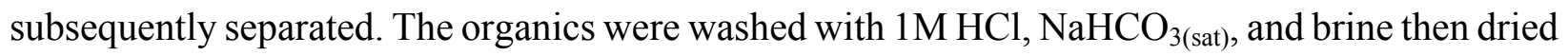
over $\mathrm{MgSO}_{4}$. Removal of the solvent afforded the biaryl ketone as a white solid (1.023 $\left.\mathrm{g}, 75 \%\right)$. ${ }^{1} \mathrm{H}$ NMR $\left(400 \mathrm{MHz}, \mathrm{CDCl}_{3}\right) \delta 7.77-7.75(\mathrm{~m}, 2 \mathrm{H}), 7.71(\mathrm{dd}, J=8.4,2.1 \mathrm{~Hz}, 1 \mathrm{H}), 7.65(\mathrm{~d}, J=2.1$ $\mathrm{Hz}, 1 \mathrm{H}), 7.18(\mathrm{~d}, J=8.4 \mathrm{~Hz}, 1 \mathrm{H}), 6.94-6.92(\mathrm{~m}, 2 \mathrm{H}), 4.11(\mathrm{q}, J=7.0 \mathrm{~Hz}, 2 \mathrm{H}), 3.95(\mathrm{~s}, 2 \mathrm{H}), 1.45$ $(\mathrm{t}, J=7.00 \mathrm{~Hz}, 3 \mathrm{H}) ;{ }^{13} \mathrm{C}\left\{{ }^{1} \mathrm{H}\right\} \mathrm{NMR}\left(100 \mathrm{MHz}, \mathrm{CDCl}_{3}\right) \delta 192.0,163.9,141.0,139.6,137.3,132.6$, $131.6,131.1,128.7,114.5,91.3,64.0,14.7$.

The synthesized ketone $(680 \mathrm{mg}, 1.76 \mathrm{mmol})$ and triethylsilane $(0.95 \mathrm{~mL}, 5.81 \mathrm{mmol})$ were dissolved in anhydrous $\mathrm{MeCN}(10 \mathrm{~mL})$ and cooled to $0{ }^{\circ} \mathrm{C}$. Borontrifluoroide diethyl etherate $(0.52$ $\mathrm{mL}, 4.22 \mathrm{mmol}$ ) was added to the reaction dropwise over 20 mins. The reaction was stirred $4 \mathrm{~h}$ at room temperature. Upon completion of the reaction, water was added and the product was extracted with EtOAc $(2 \times 10 \mathrm{~mL})$. The combined organics were washed with water $(3 \times 10 \mathrm{~mL})$ and dried over $\mathrm{MgSO}_{4}$. Removal of the solvent afforded the crude product as an oil. The product was purified by flash chromatography using $0-10 \%$ EtOAc in hexanes to furnish the product as a white solid (362.5 mg, 55\%). Spectroscopic data is consistent with a previous report. ${ }^{14}{ }^{1} \mathrm{H} \mathrm{NMR}$ $\left(400 \mathrm{MHz}, \mathrm{CDCl}_{3}\right) \delta$ 7.46-7.43 (m, 2H), 7.08-7.05 (m, 3H), 6.84-6.81 (m, 2H), 4.00 (q, J= 7.0

14 Zhao, M. M.; Zhang, H.; Iimura, S.; Bednarz, M. S.; Song, Q.-L.; Lim, N.-K.; Yan, J.; Wu, W.; Dai, K.; Gu, X.; Wang, Y. Org. Process Res. Dev. 2020, 24 (11), 2689-2701. 
$\mathrm{Hz}, 2 \mathrm{H}), 3.95(\mathrm{~s}, 2 \mathrm{H}), 1.40(\mathrm{t}, J=7.00 \mathrm{~Hz}, 3 \mathrm{H}) ;{ }^{13} \mathrm{C}\left\{{ }^{1} \mathrm{H}\right\} \mathrm{NMR}\left(100 \mathrm{MHz}, \mathrm{CDCl}_{3}\right) \delta 157.6,141.5$, $139.5,136.5,134.2,131.1,130.4,129.9,114.6,91.6,63.4,38.0,14.9$; IR (neat) $v_{\max }: 3062-3029$ (CH, aromatic), 2918-2870 (C-H, aliphatic), $1746(\mathrm{C}=\mathrm{O}$, lactone $), 1095,732,694 \mathrm{~cm}^{-1}$.

(11) 3,4,5,7-Tetra-O-benzyl-2,6-anhydro-1-(4-chloro-3-(4-ethoxybenzyl)phenyl)-1-deoxy-D-glucohept-1-enitol<smiles>CCOc1ccc(Cc2cc(/C=C3\OC(COCc4ccccc4)[C@@H](OCc4ccccc4)[C@H](OCc4ccccc4)C3OCc3ccccc3)ccc2Cl)cc1</smiles>

The title compound was prepared from exo-glucal 5 (40 mg, $0.075 \mathrm{mmol}$ ) and aryl iodide 10 (55 $\mathrm{mg}, 0.150 \mathrm{mmol}$ ) following general procedure 2.1 for a duration of $24 \mathrm{~h}$. Purification by column chromatography using $10 \%$ ethyl acetate in hexanes afforded 11 as a white solid (87 \%, $51 \mathrm{mg})$. ${ }^{1} \mathrm{H}$ NMR $\left(400 \mathrm{MHz}, \mathrm{CDCl}_{3}\right) \delta 7.55(\mathrm{dd}, J=8.4,2.1 \mathrm{~Hz}, 1 \mathrm{H}), 7.43(\mathrm{~d}, J=2.0 \mathrm{~Hz}, 1 \mathrm{H}), 7.36-7.15$ $(\mathrm{m}, 21 \mathrm{H}), 7.08(\mathrm{~d}, J=8.6 \mathrm{~Hz}, 2 \mathrm{H}), 6.80-6.78(\mathrm{~m}, 2 \mathrm{H}), 5.63(\mathrm{~s}, 1 \mathrm{H}), 4.78-4.73(\mathrm{~m}, 3 \mathrm{H}), 4.64(\mathrm{~d}, J$ $=11.4 \mathrm{~Hz}, 1 \mathrm{H}), 4.59(\mathrm{~d}, J=12.0 \mathrm{~Hz}, 1 \mathrm{H}), 4.54-4.50(\mathrm{~m}, 2 \mathrm{H}), 4.07-4.04(\mathrm{~m}, 1 \mathrm{H}), 4.00-3.92(\mathrm{~m}$, 5H), 3.83-3.80 (m, 2H), 3.75-3.74 (m, 2H), $1.36(\mathrm{t}, J=7.0 \mathrm{~Hz}, 3 \mathrm{H})$; $\mathrm{H}^{1}-\mathrm{H}^{3}$ nOe observed 2\%; ${ }^{13} \mathrm{C}\left\{{ }^{1} \mathrm{H}\right\} \mathrm{NMR}\left(100 \mathrm{MHz}, \mathrm{CDCl}_{3}\right) \delta 157.0,149.7,138.6,138.2,138.2,137.9,134.1,131.9,131.8$, $131.4,130.0,129.4,128.6,128.5,128.5,128.5,128.4,128.0,128.0,127.9,127.8,127.8,114.5$, $108.6,84.5,79.3,77.0,74.1,73.65,73.57,72.0,69.1,63.4,38.5,15.0$; IR (neat) $v_{\max }: 2979-2925$ (C-H, aliphatic), 1656, 1595, 735, $696 \mathrm{~cm}^{-1}$; HRMS (DART) $\mathrm{m} / z$ : $\left[\mathrm{M}+\mathrm{NH}_{4}\right]^{+}$Calcd for $\mathrm{C}_{50} \mathrm{H}_{53} \mathrm{NO}_{6} \mathrm{Cl} 798.35559$; found 798.35513.

(12) 2,6-anhydro-1-deoxy-1-(4-chloro-3-(4-ethoxybenzyl)phenyl)-D-glycero-L-gulo-heptitol

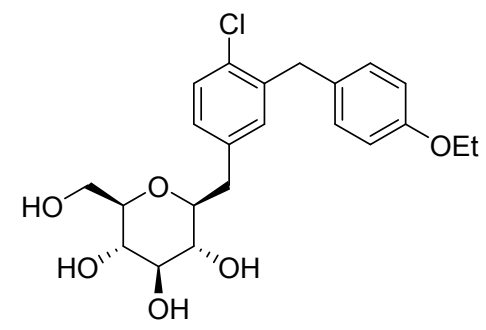

A solution of aryl-exo-glycal (11) $(35 \mathrm{mg}, 0.045 \mathrm{mmol})$ in anhydrous $\mathrm{MeOH} / \mathrm{EtOAc}(1: 1,1 \mathrm{~mL})$ was degassed by purging with argon for $1 \mathrm{~h}$. $\mathrm{Pd} / \mathrm{C} 10 \% \mathrm{wt}(7 \mathrm{mg}, 15 \mathrm{~mol} \%)$ was added and the atmosphere was replaced with hydrogen. The reaction was stirred at room temperature for $24 \mathrm{~h}$ and then was filtered through celite. The solvent was removed from the filtrate to afford the crude product as a colourless oil. The product was isolated by flash chromatography $(0-10 \% \mathrm{MeOH}$ in DCM) to afford 12 as a colourless oil (15.5 mg, 82\%). Spectroscopic data is consistent with a 
previous report. ${ }^{15}{ }^{1} \mathrm{H}$ NMR $\left(400 \mathrm{MHz}, \mathrm{CD}_{3} \mathrm{OD}\right) \delta$ 7.25-7.08 (m, 5H); 6.84-6.80 (m, 2H); 4.03$3.98(\mathrm{~m}, 4 \mathrm{H}) ; 3.75(\mathrm{dd}, J=11.8,2.4 \mathrm{~Hz}, 1 \mathrm{H}) ; 3.61(\mathrm{dd}, J=11.8,5.4 \mathrm{~Hz}, 1 \mathrm{H}) ; 3.35-3.24$ (m, 4H); 3.13-3.06 (m, 3H); $2.68(\mathrm{dd}, \mathrm{J}=14.5,8.4 \mathrm{~Hz}, 1 \mathrm{H}) ; 1.37(\mathrm{t}, J=7.0 \mathrm{~Hz}, 3 \mathrm{H}) ;{ }^{13} \mathrm{C}\left\{{ }^{1} \mathrm{H}\right\} \mathrm{NMR}(100$ $\left.\mathrm{MHz}, \mathrm{CDCl}_{3}\right) \delta 158.8,139.7,139.4,133.6,133.1,132.7,130.8,130.2,129.9,115.4,81.3,79.9$, 74.8, 71.8, 64.4, 62.9, 39.1, 38.0, 15.2; IR (neat) $v_{\max }$ : $3365(\mathrm{OH}), 3062-3029(\mathrm{CH}$, aromatic), 2978-2919 (C-H, aliphatic), 2490 (br), 1509, 1240, 1038, $818 \mathrm{~cm}^{-1}$.

15. Mukkamala, R.; Kumar, R.; Banerjee, S. K.; Aidhen, I. S. Eur. J. Org. Chem. 2020, 2020(12), 1828-1839. 


\section{4. ${ }^{1} \mathrm{H}$ and ${ }^{13} \mathrm{C}\left\{{ }^{1} \mathrm{H}\right\}$ NMR Spectra}

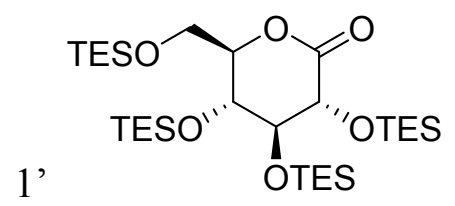

1H NMR (400 MHz, CDCl3)
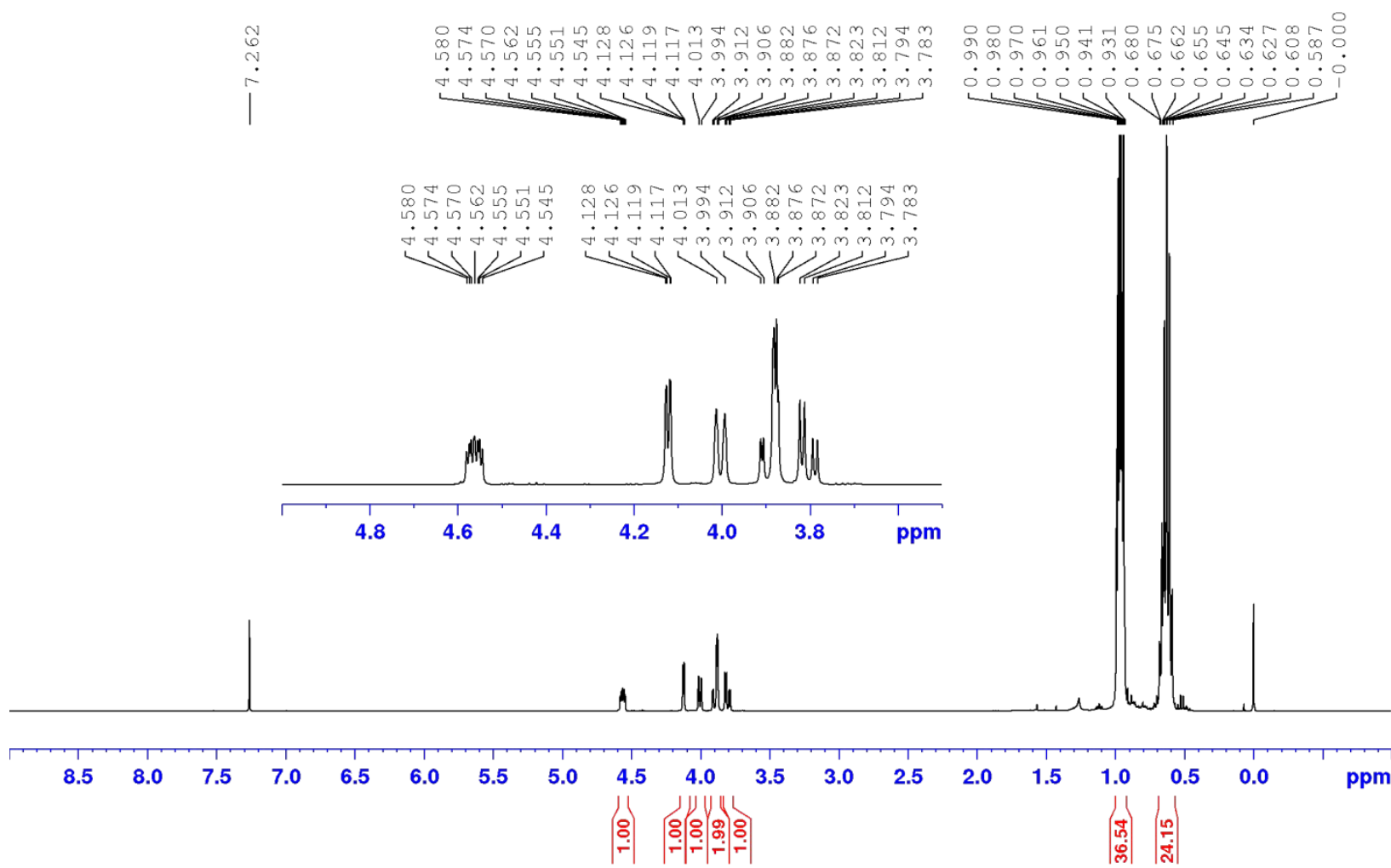


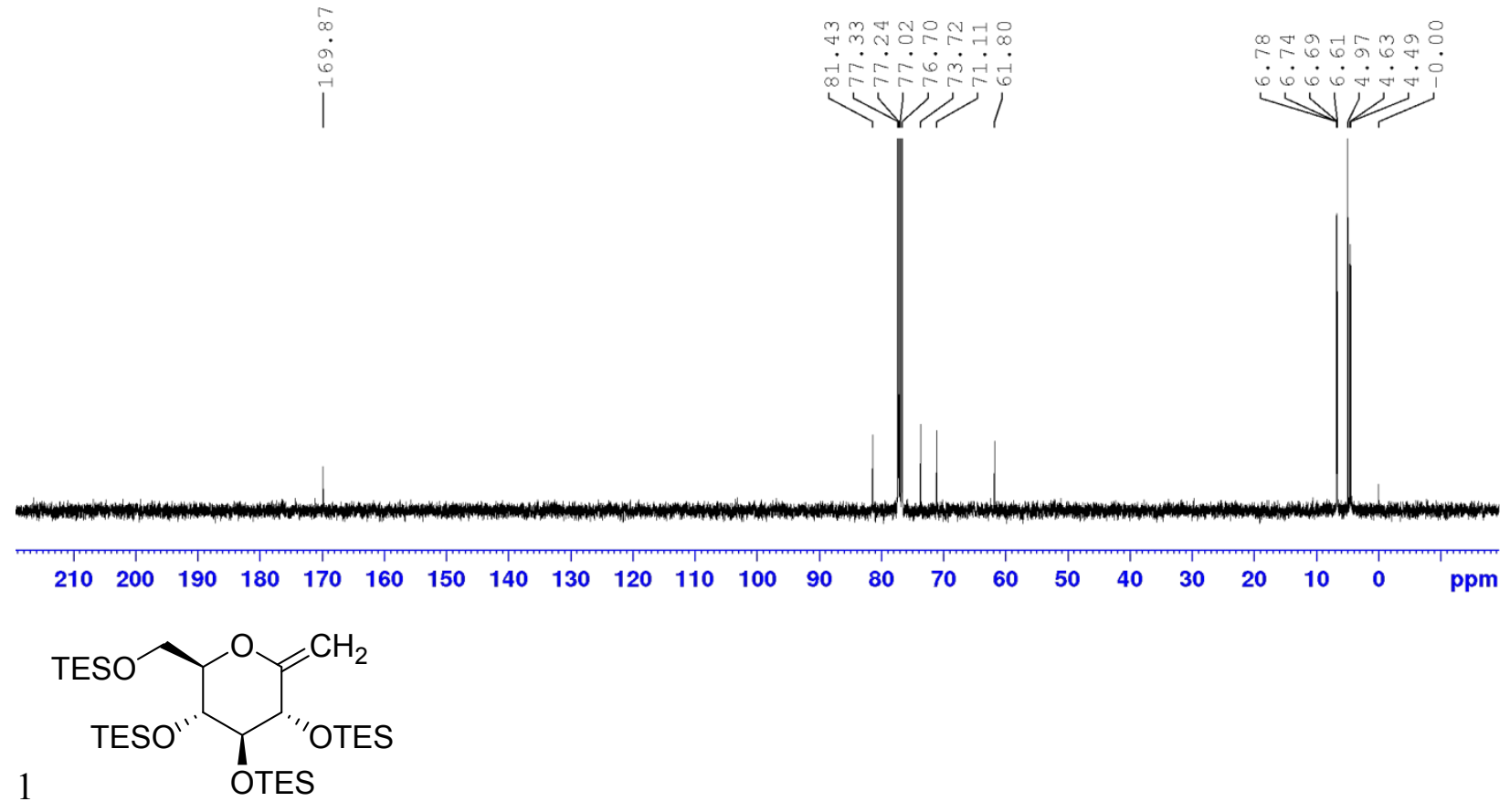

1H NMR (400 MHz, CDCl3)

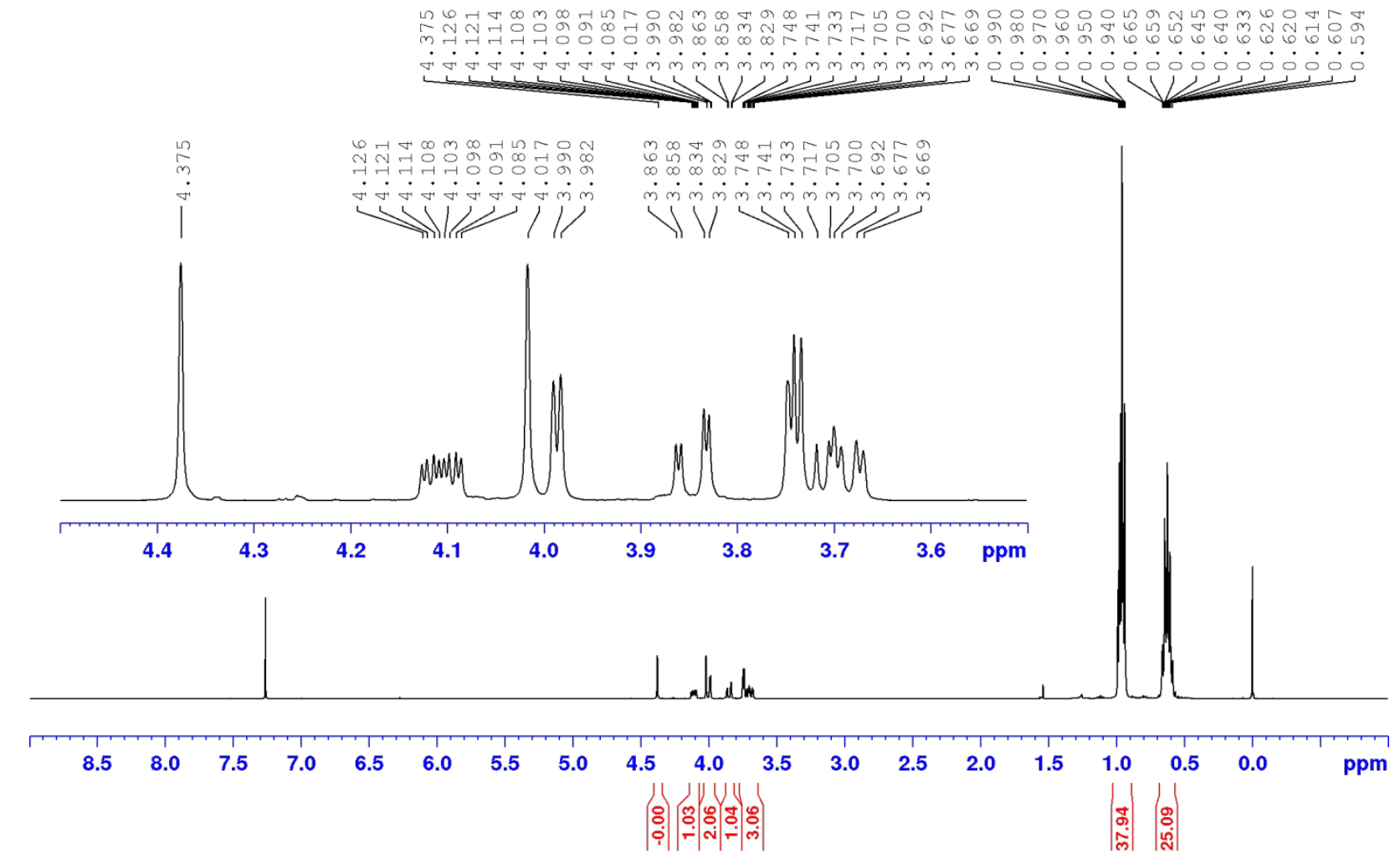



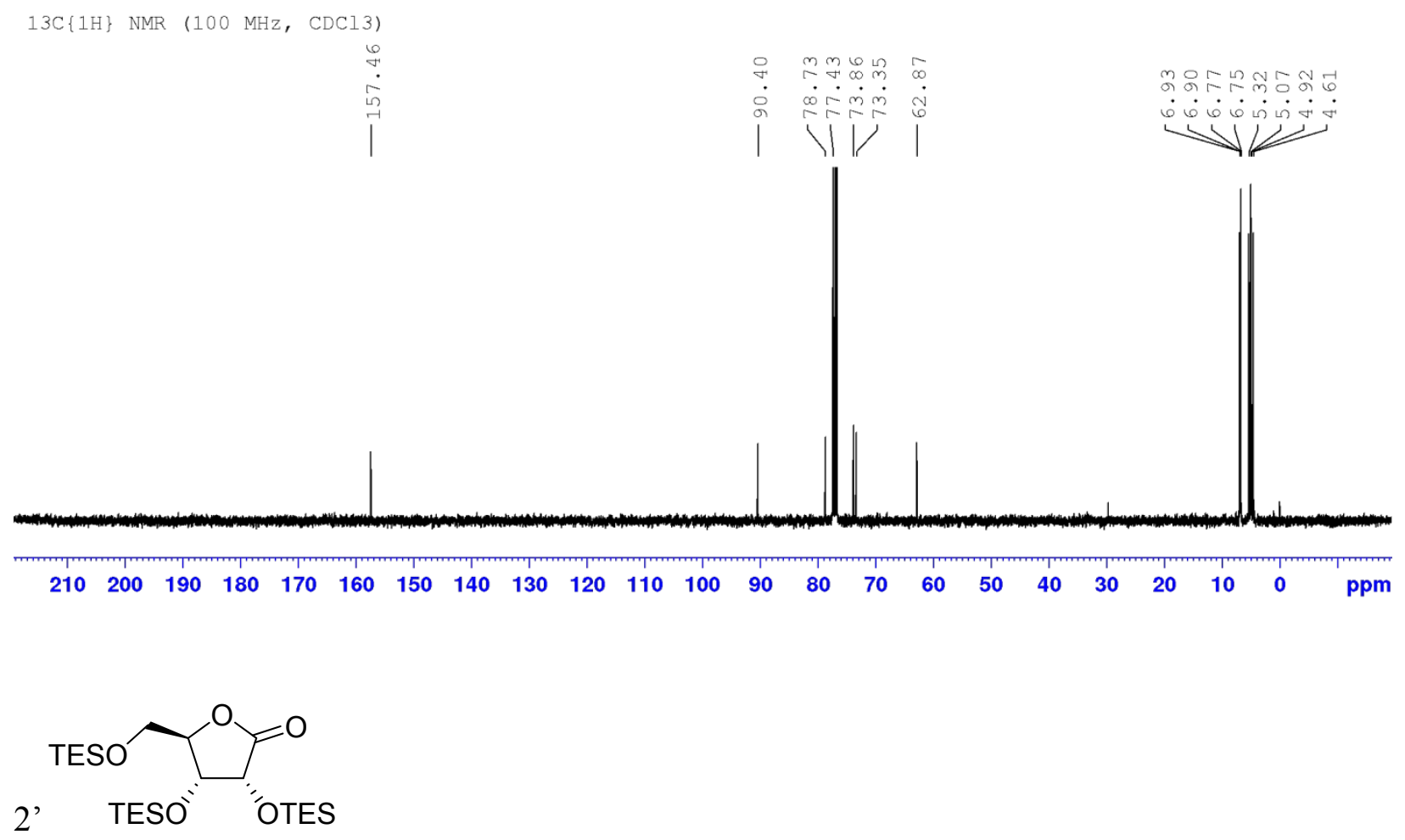

1H NMR (400 MHz, CDCl3)

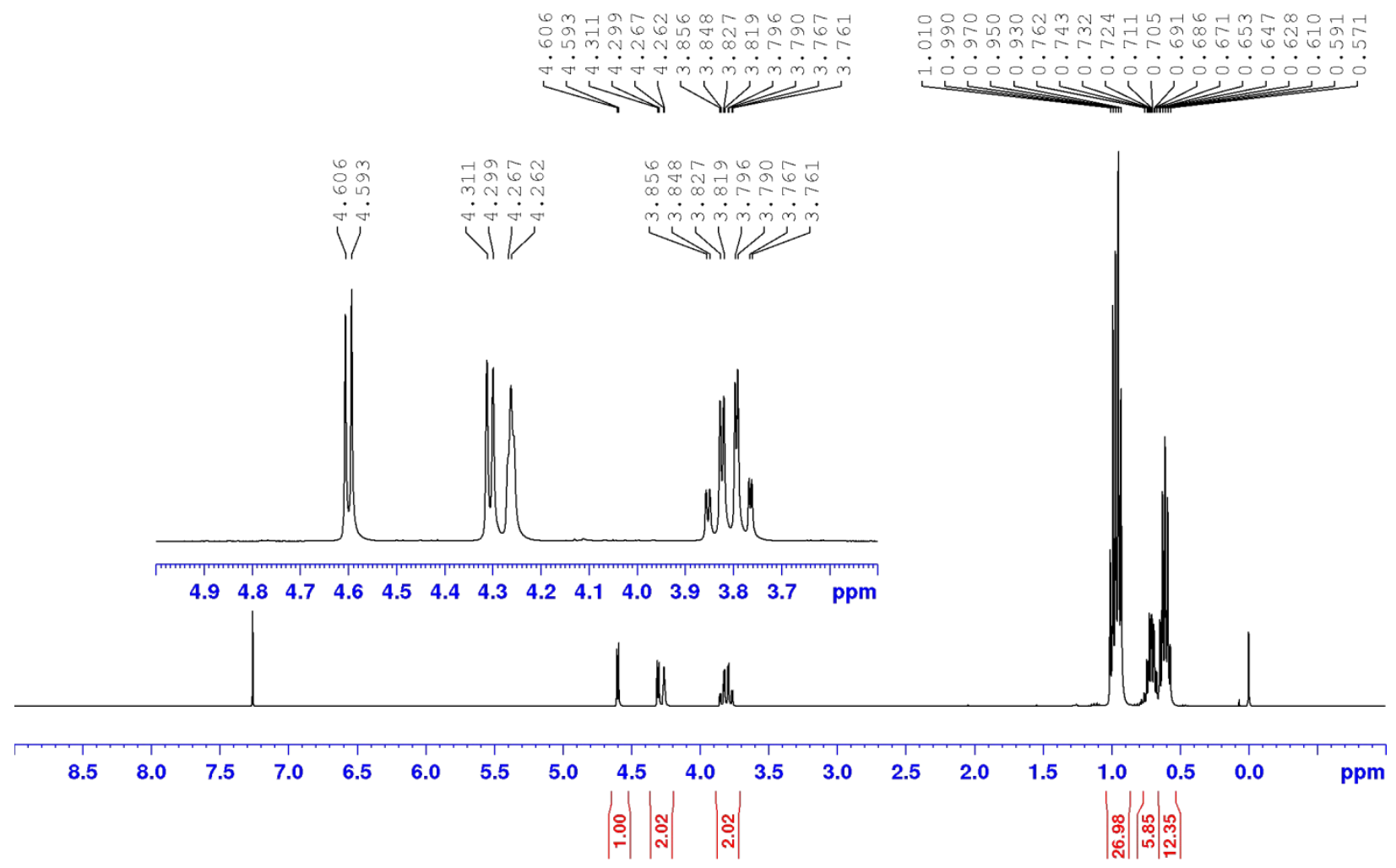



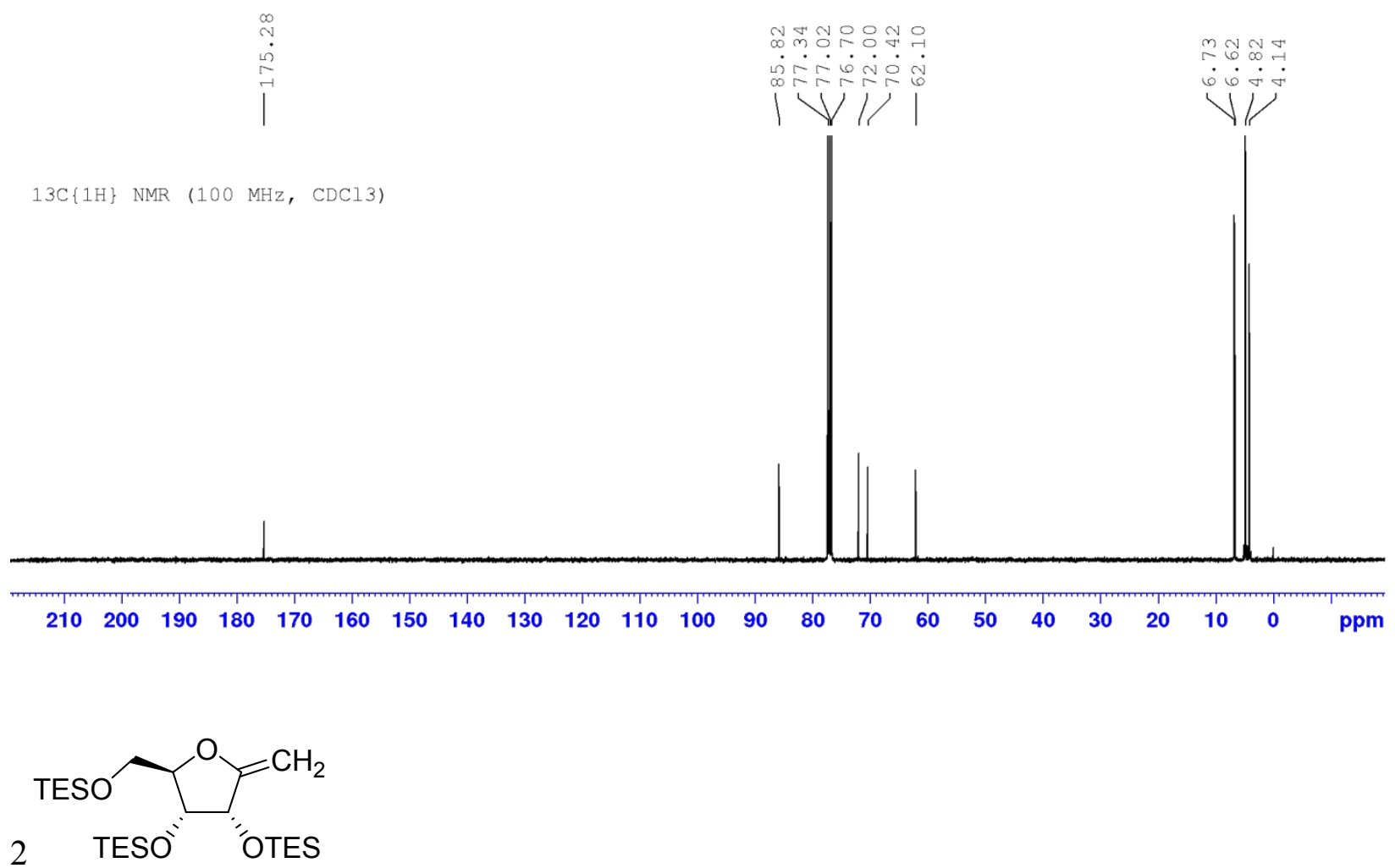

$1 \mathrm{H} \operatorname{NMR}(400 \mathrm{MHz}, \mathrm{CDCl} 3)$
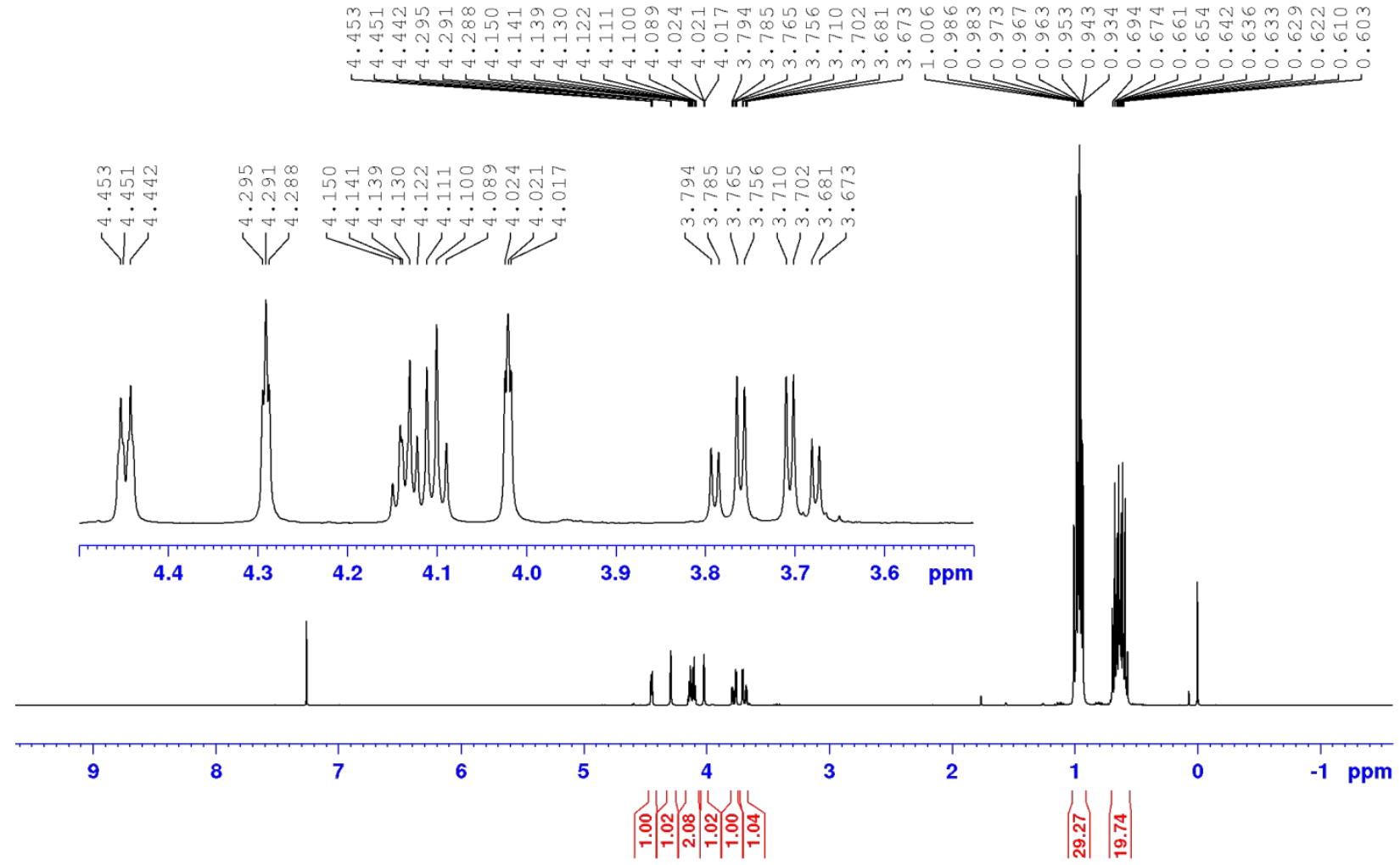

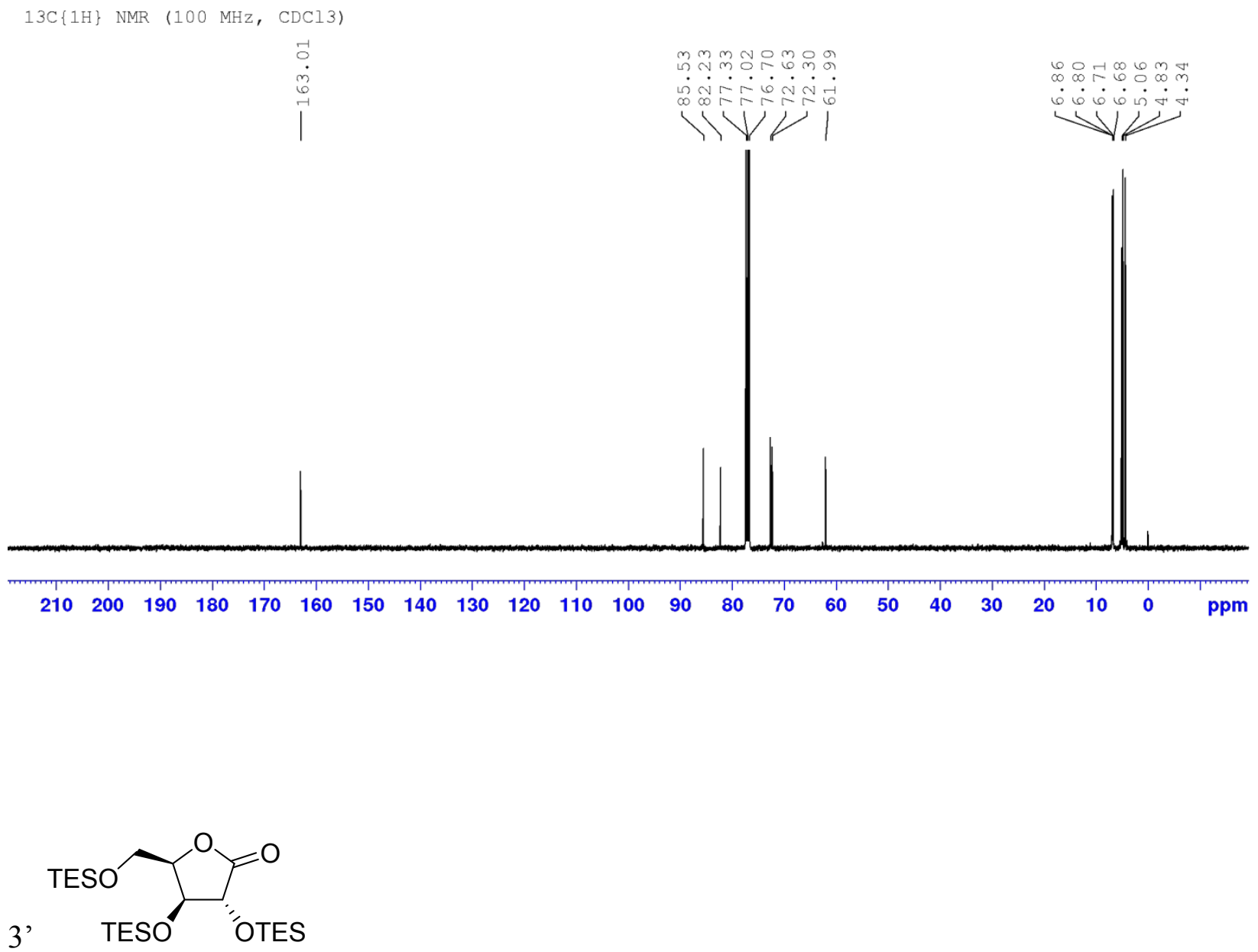

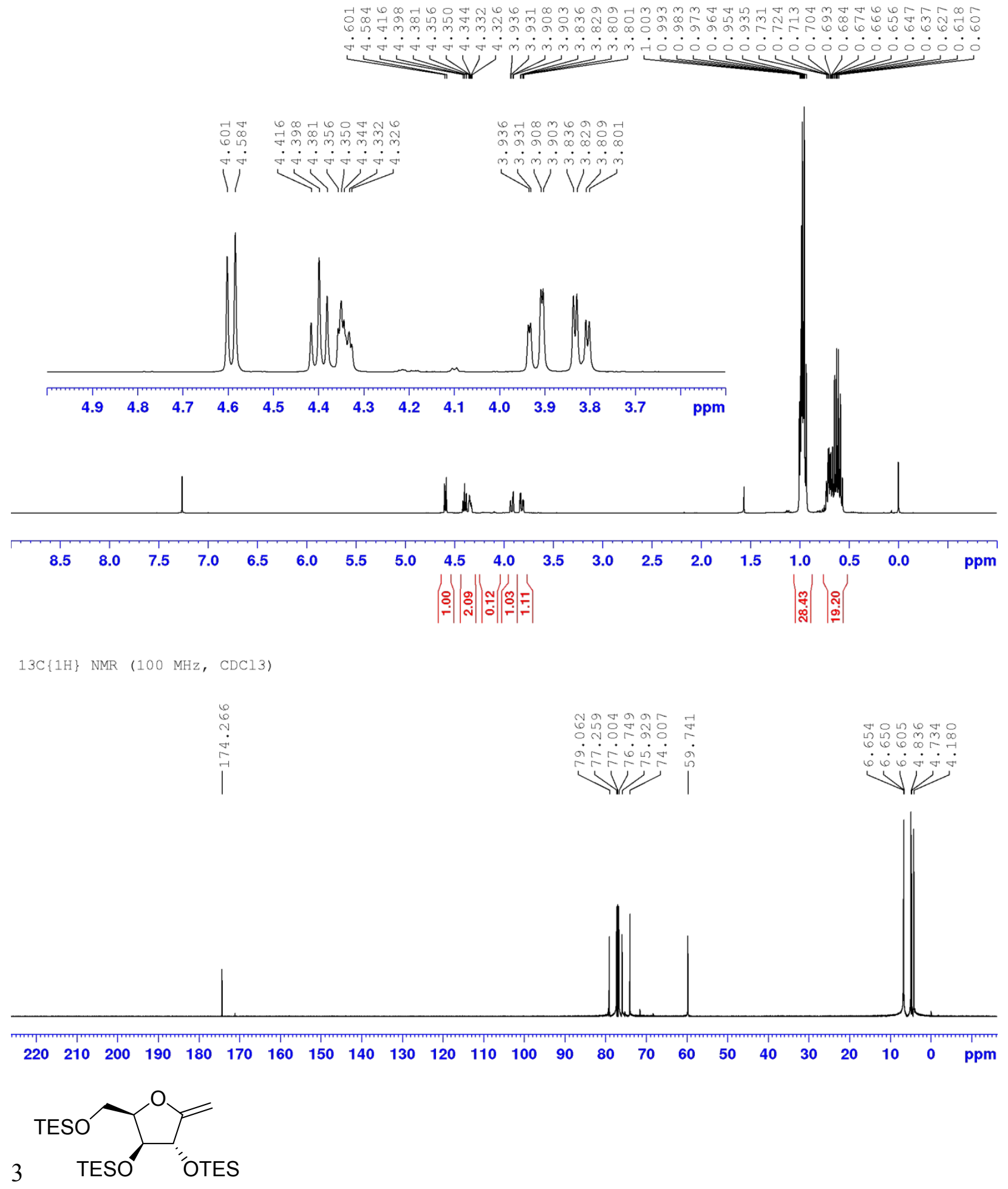


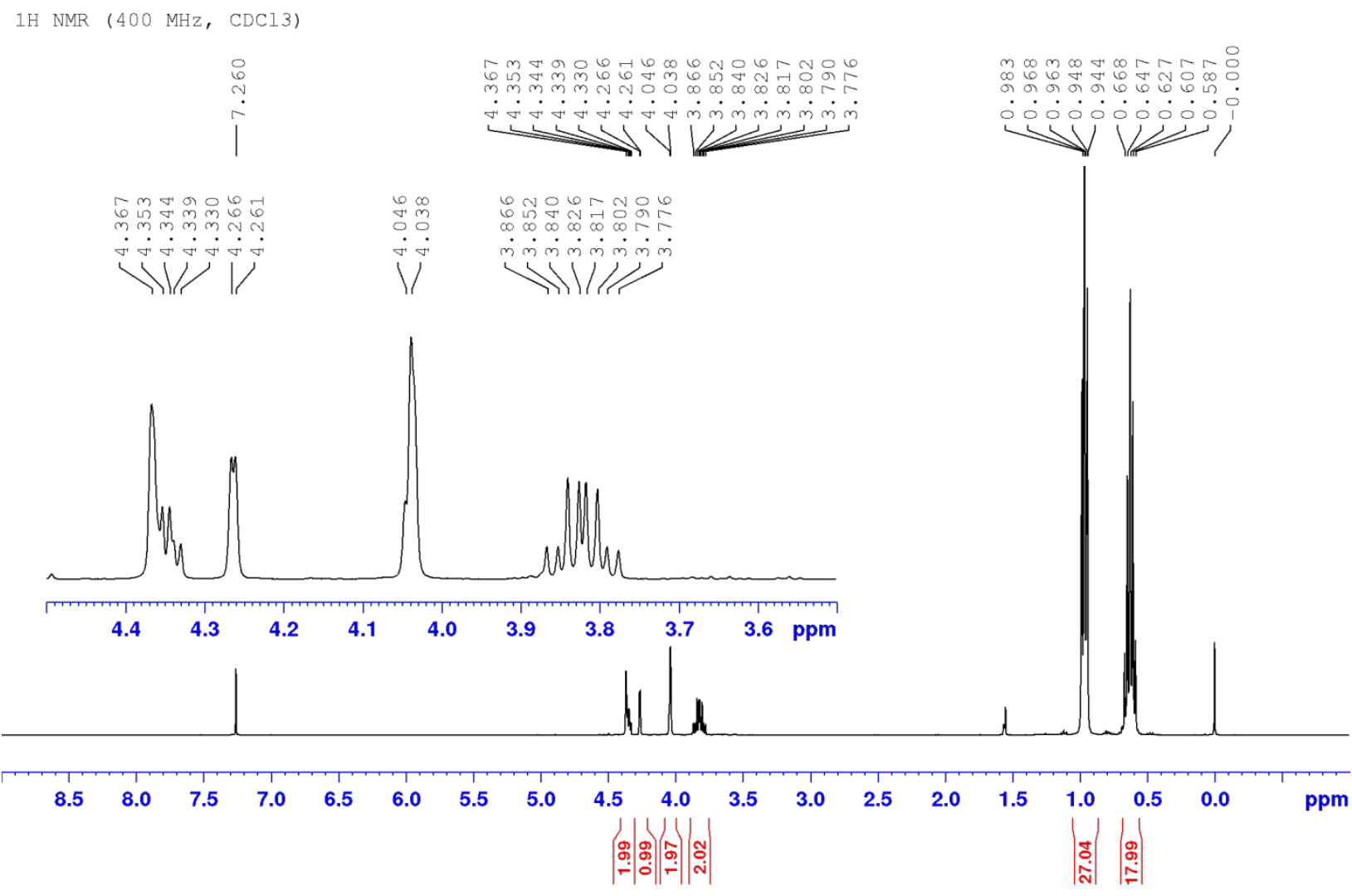

13C $\{1 \mathrm{H}\}$ NMR (100 MHz, CDCl3)

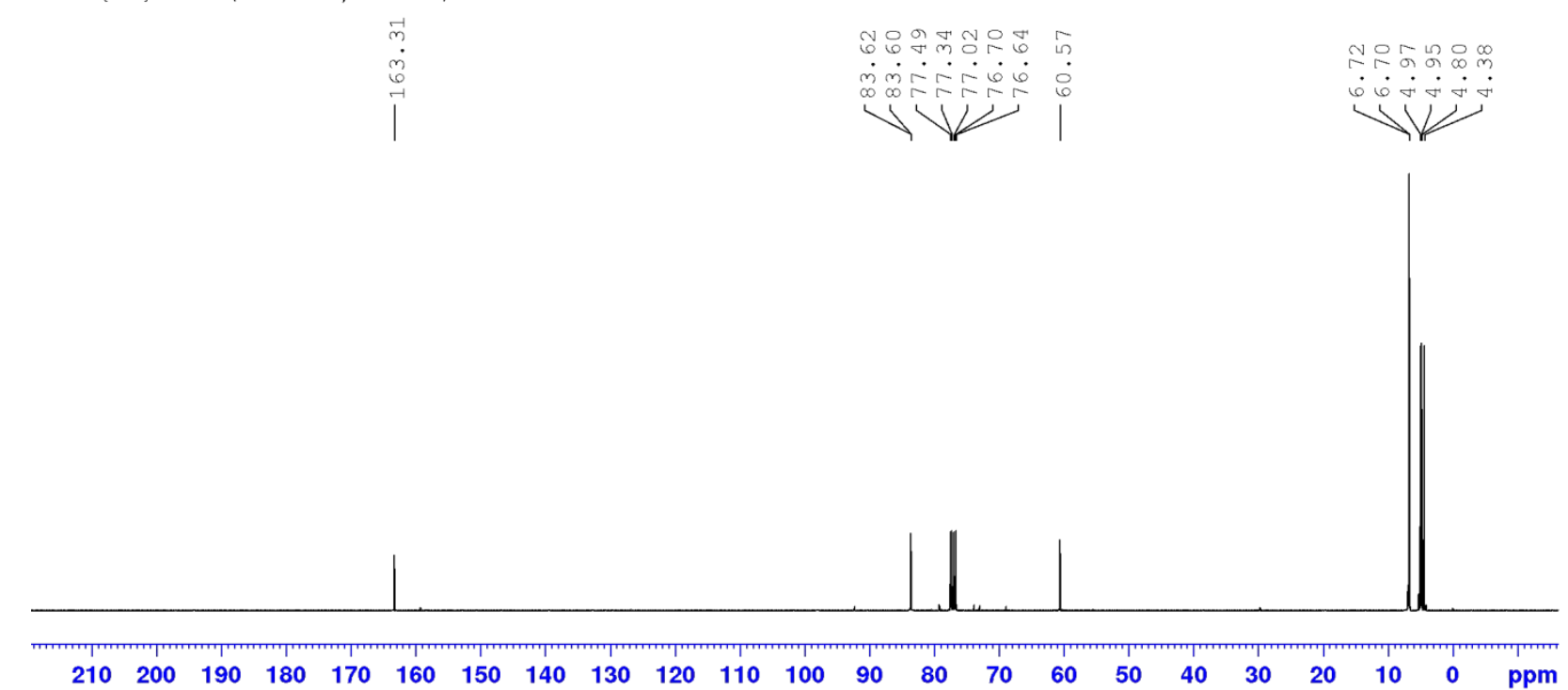




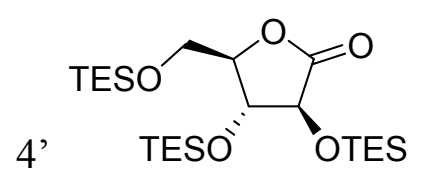

1H NMR (400 MHz, CDCl3)

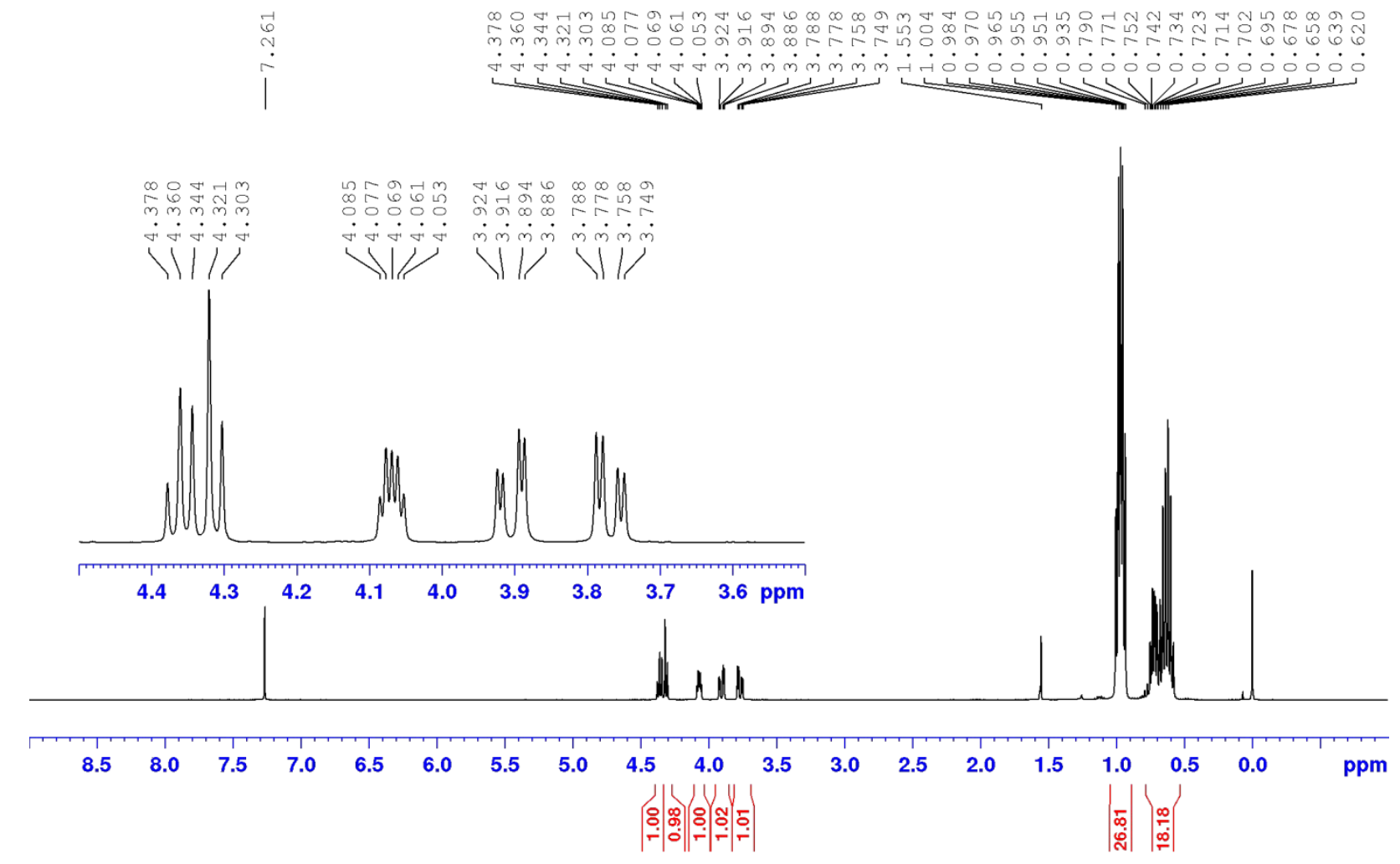

$13 \mathrm{C}\{1 \mathrm{H}\} \mathrm{NMR}(100 \mathrm{MHz}, \mathrm{CDCl} 3)$

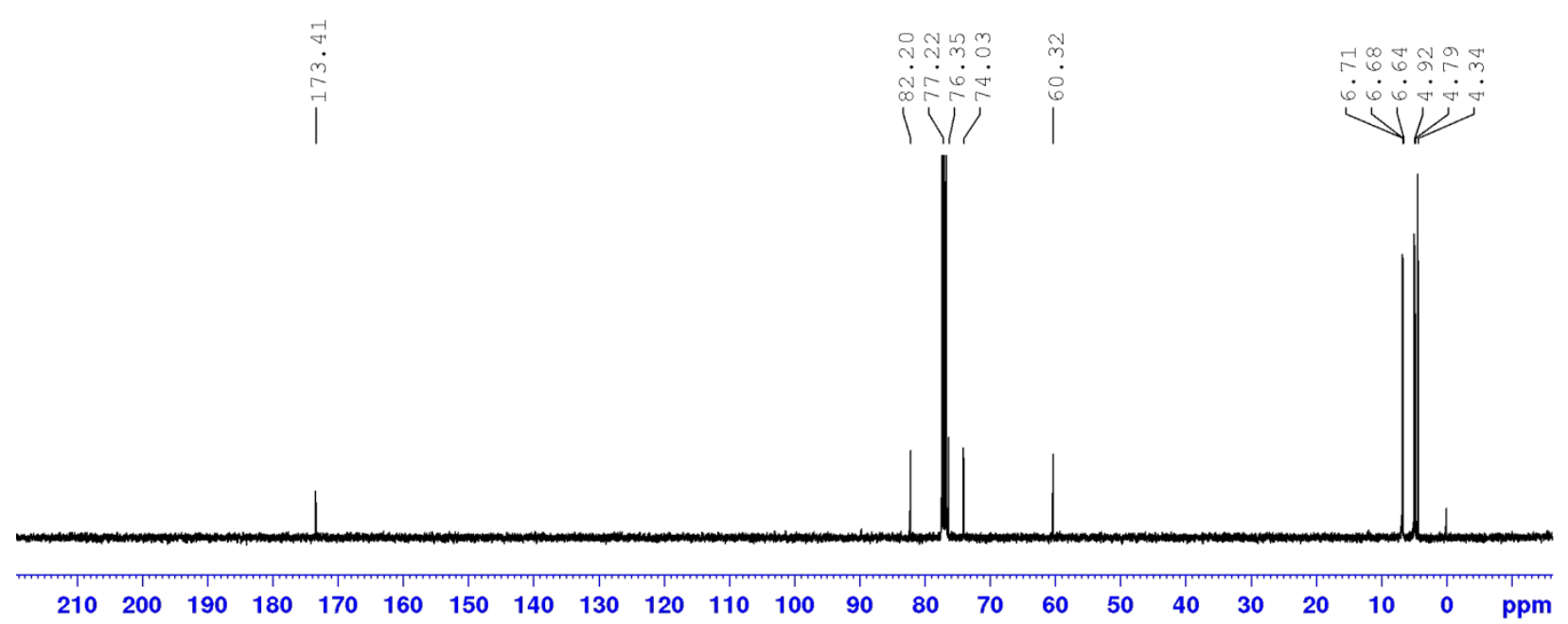




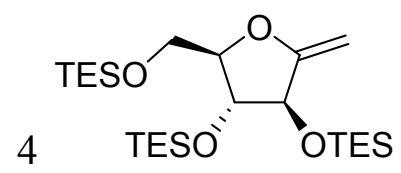

$1 \mathrm{H}$ NMR (400 MHz, CDCl3)

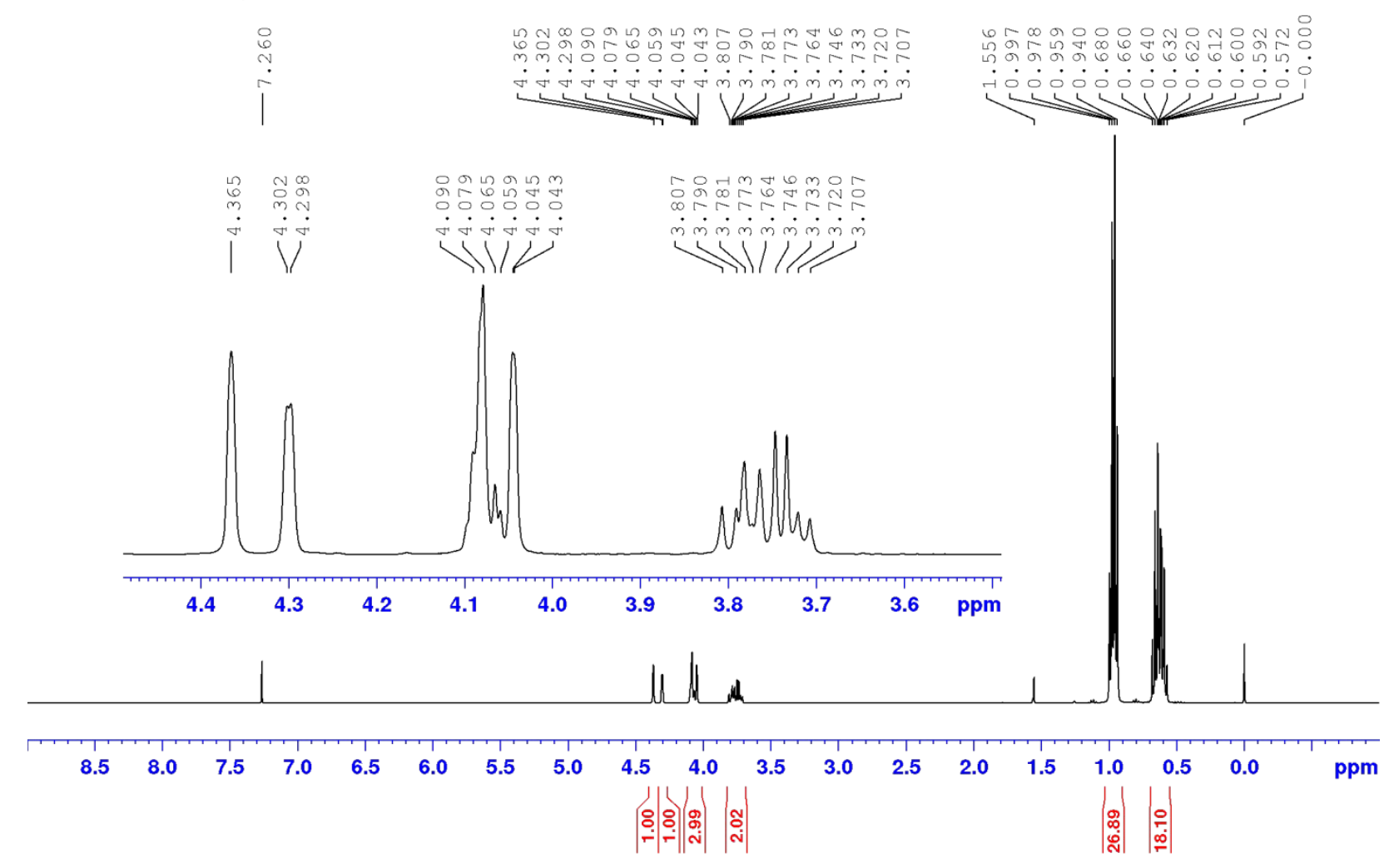

$13 \mathrm{C}\{1 \mathrm{H}\} \mathrm{NMR}(100 \mathrm{MHz}, \mathrm{CDCl} 3)$

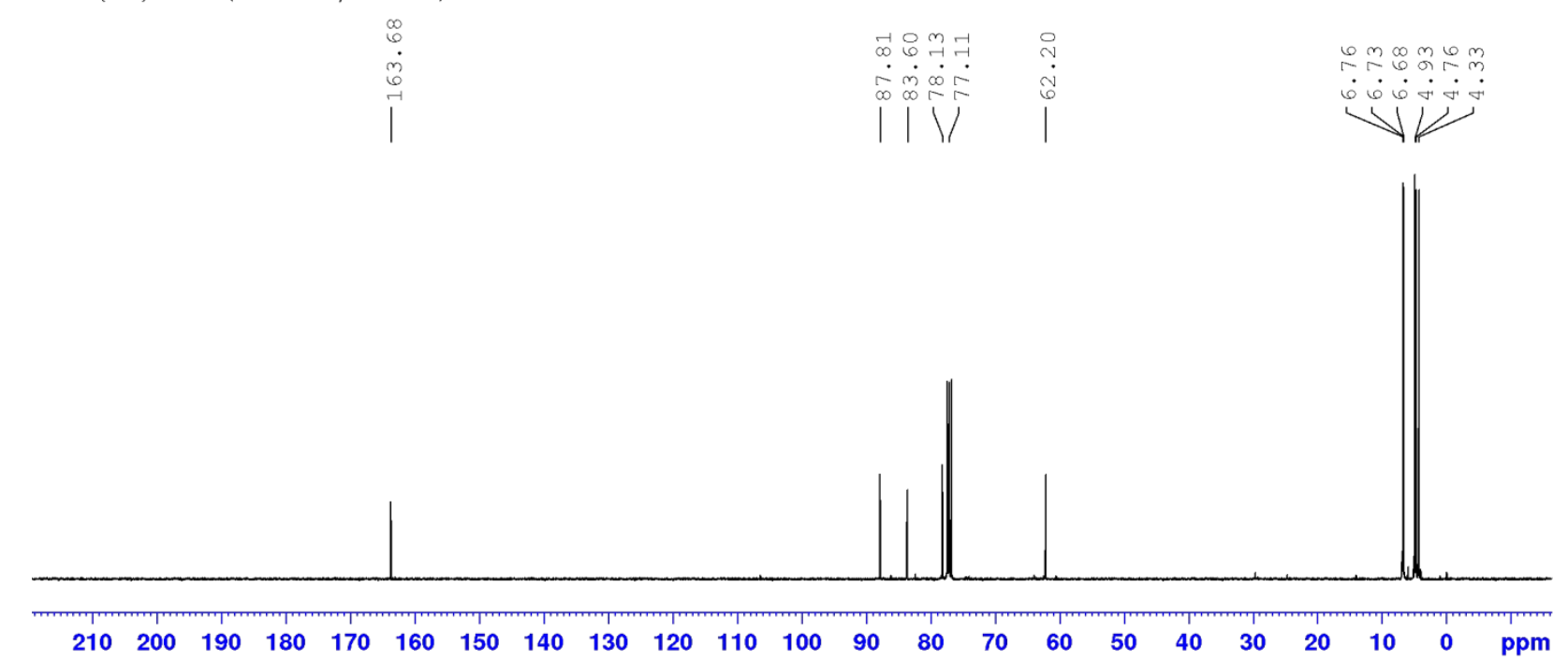




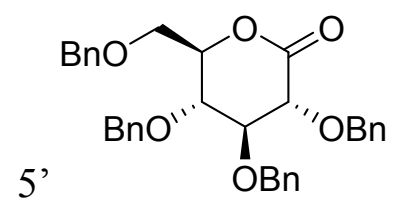

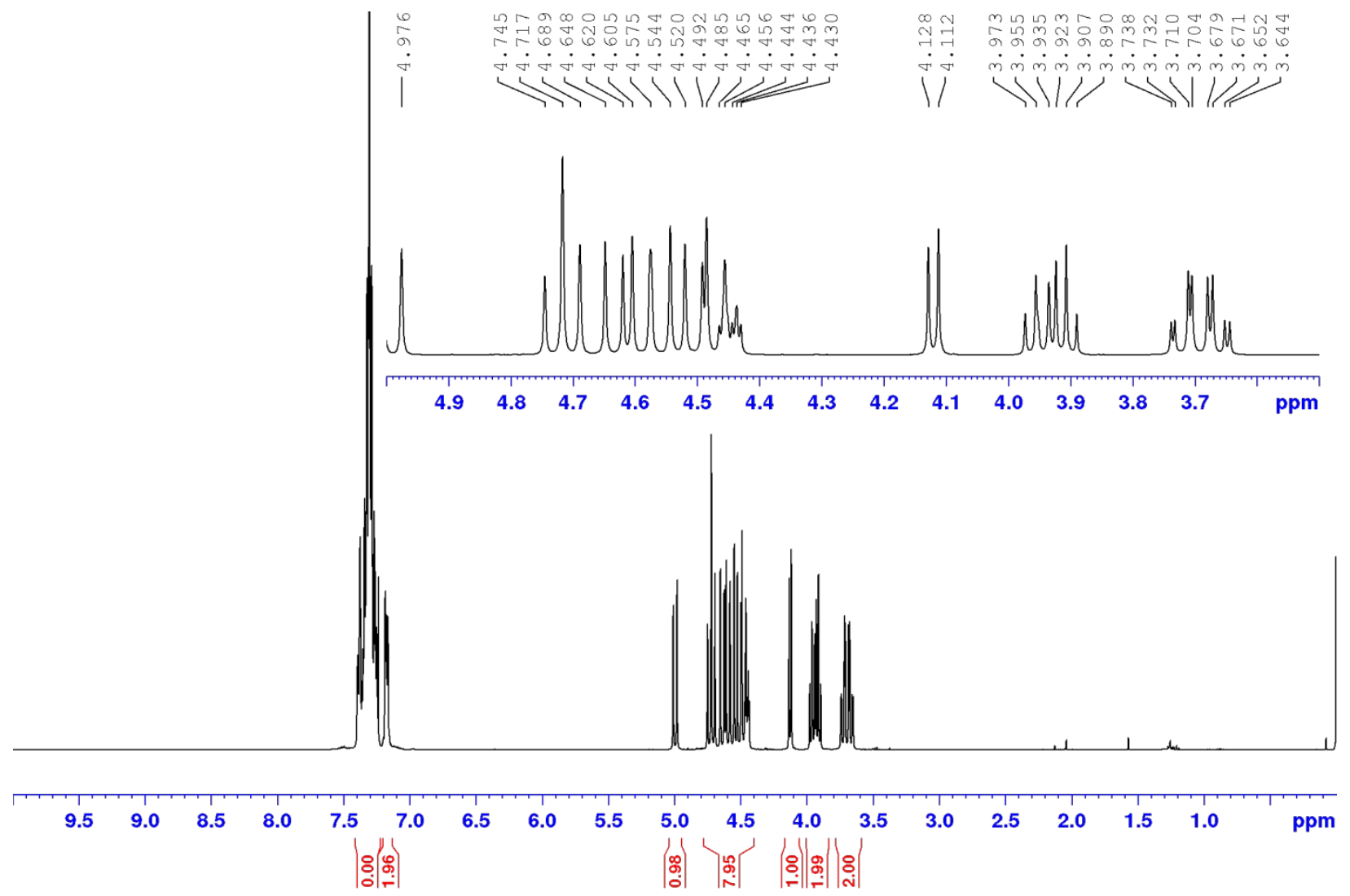

$13 \mathrm{C}\{1 \mathrm{H}\} \operatorname{NMR}(100 \mathrm{MHz}, \mathrm{CDCl} 3)$

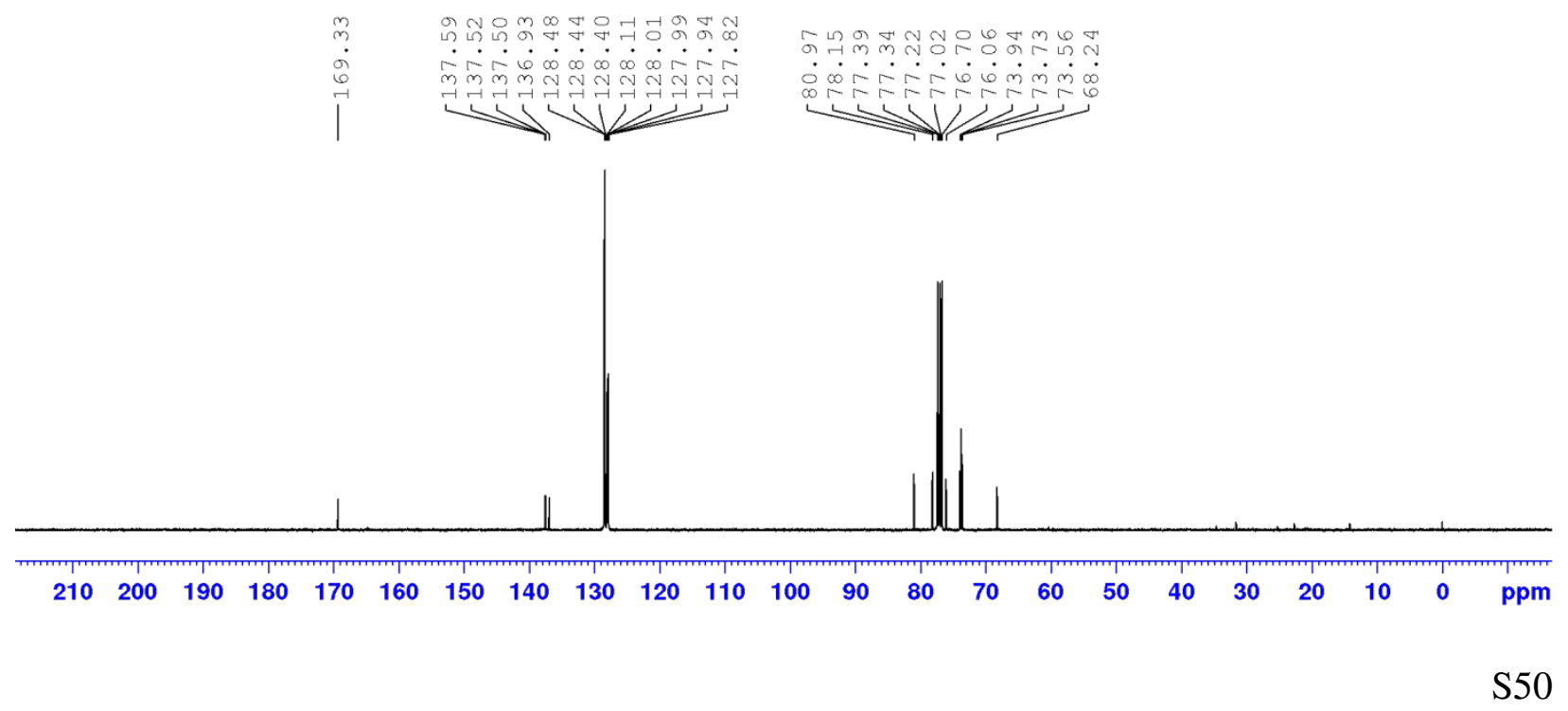



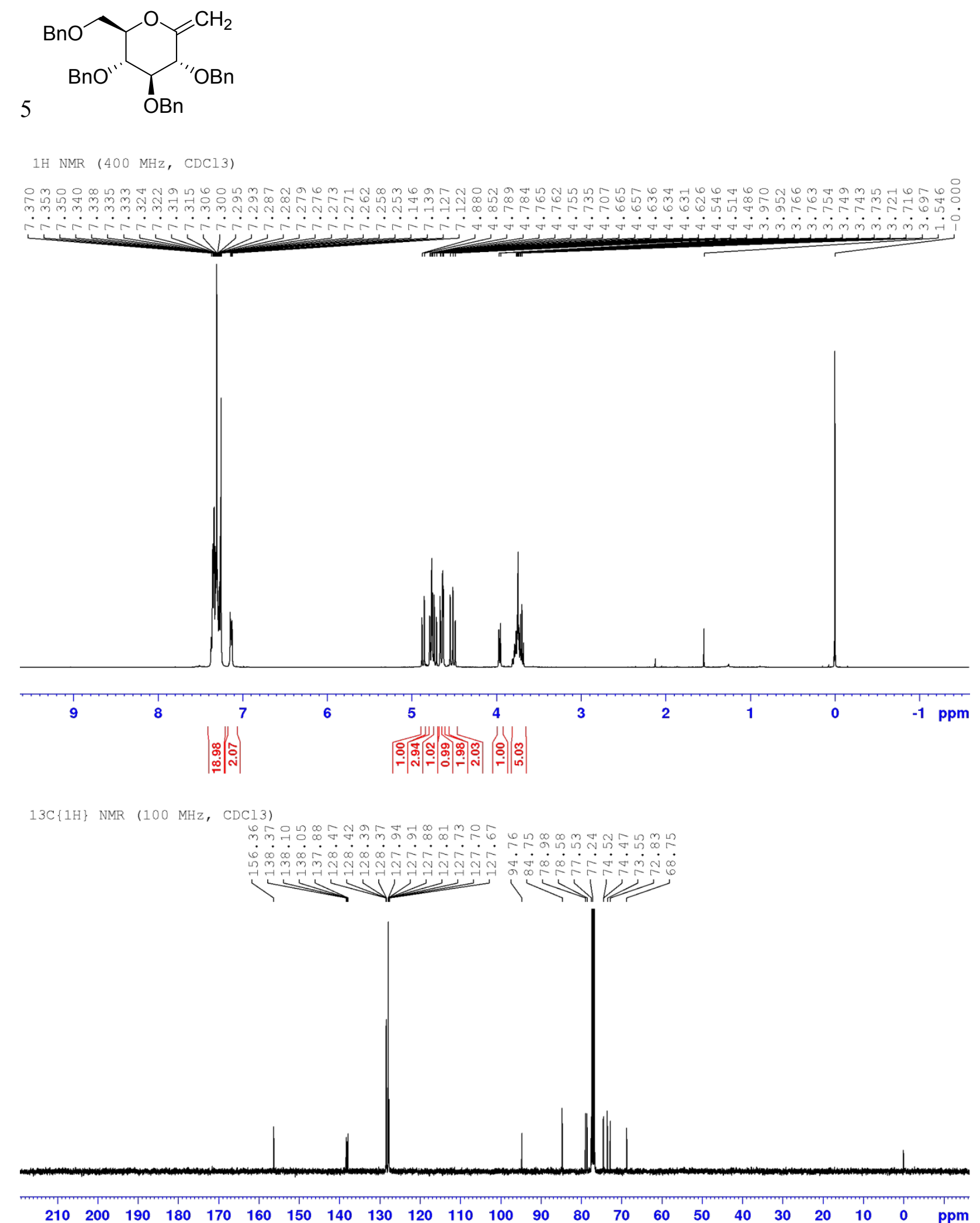


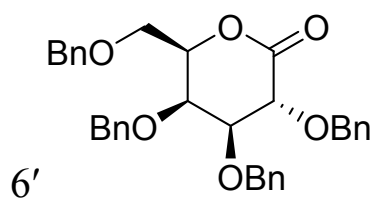

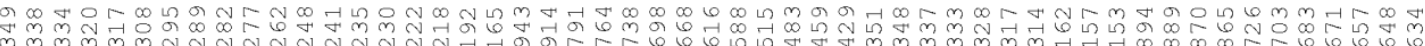

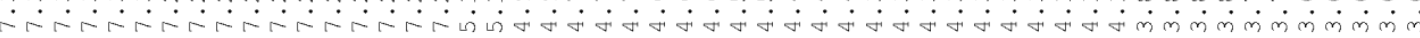

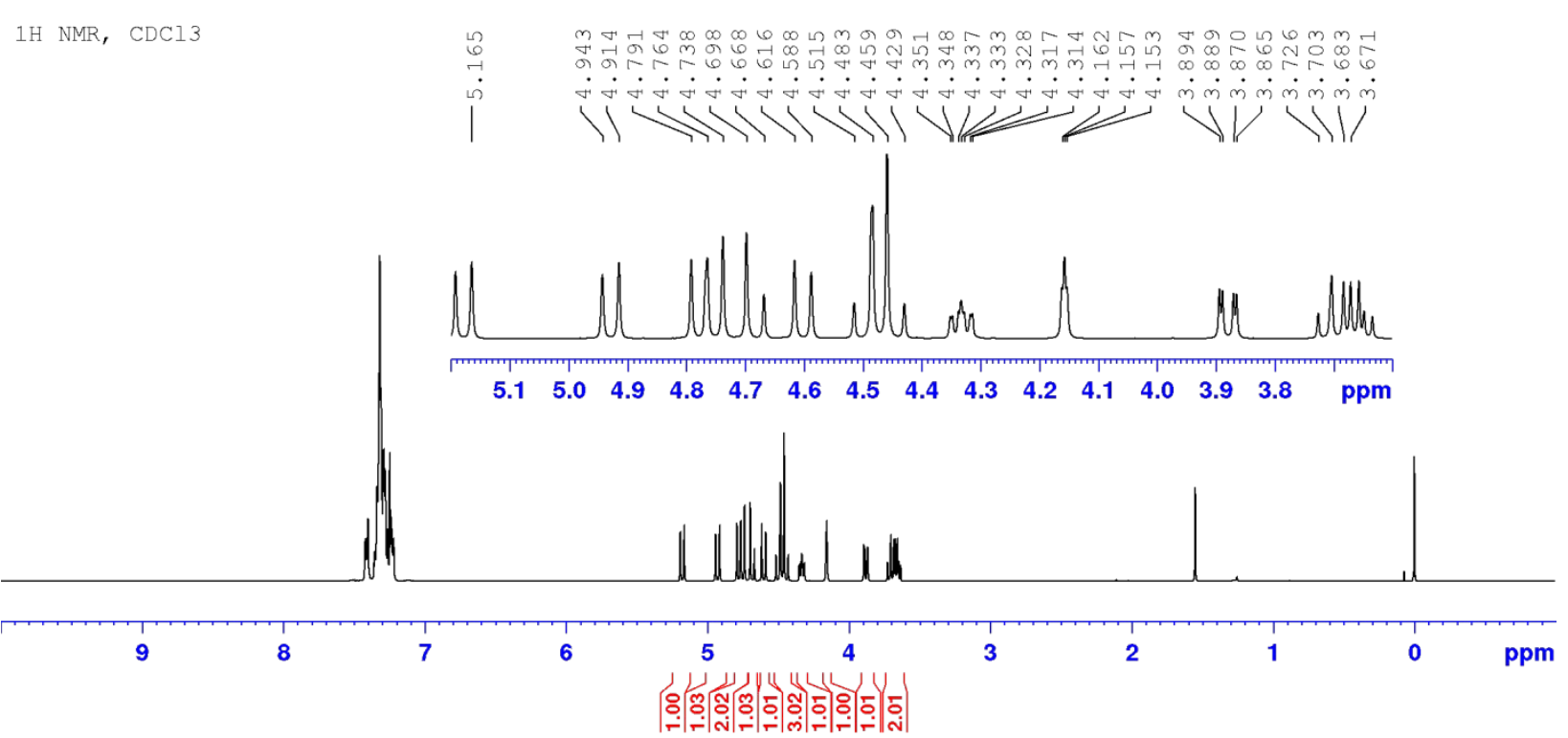

$13 \mathrm{C}\{1 \mathrm{H}\} \operatorname{NMR}(100 \mathrm{MHz}, \mathrm{CDCl} 3)$

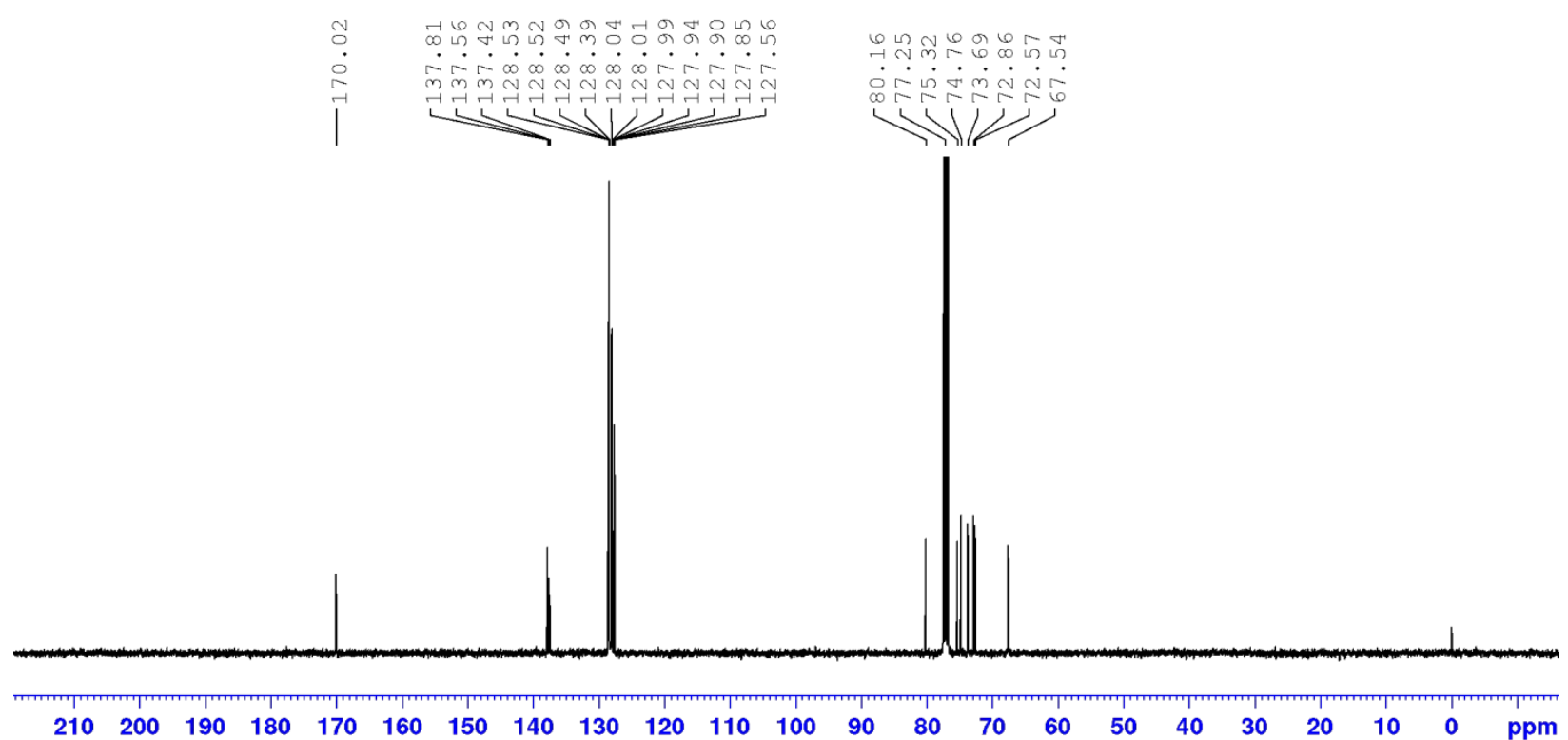


<smiles>C=C1OC(COBr)[C@H](Br)[C@H](O[C@H](Br)c2ccccc2)[C@H]1OCc1ccccc1</smiles>

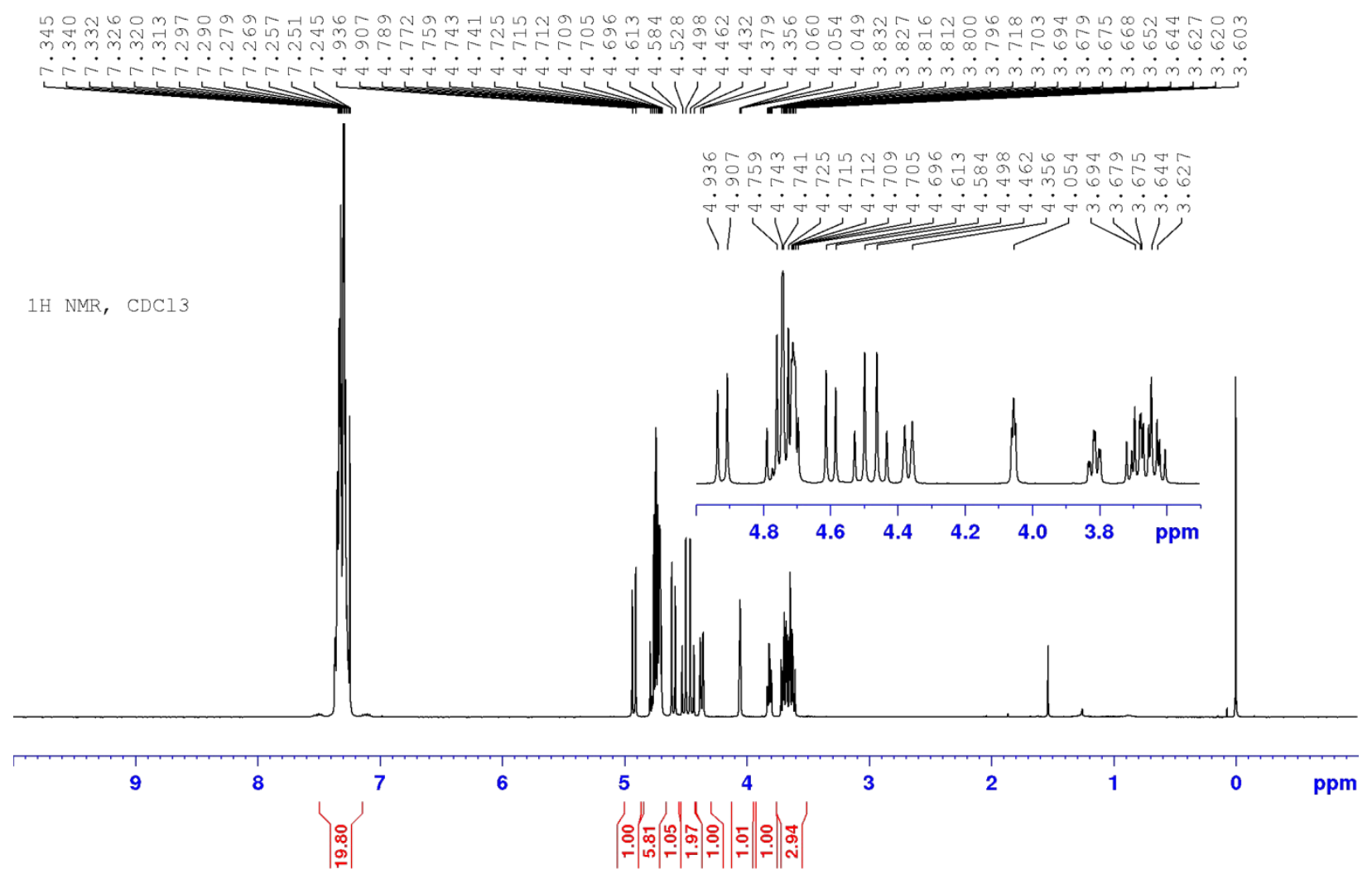

$13 \mathrm{C}\{1 \mathrm{H}\} \mathrm{NMR}(100 \mathrm{MHz}, \mathrm{CDCl} 3)$

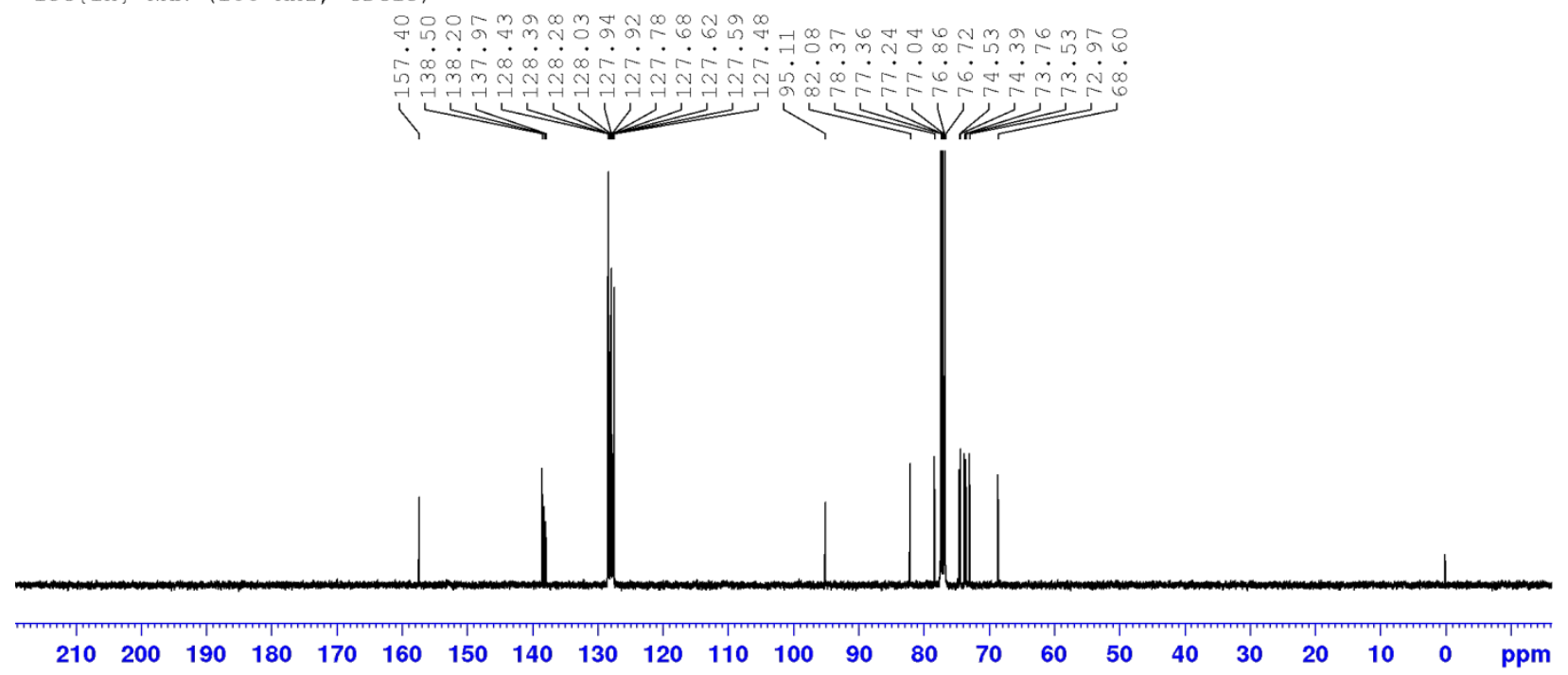



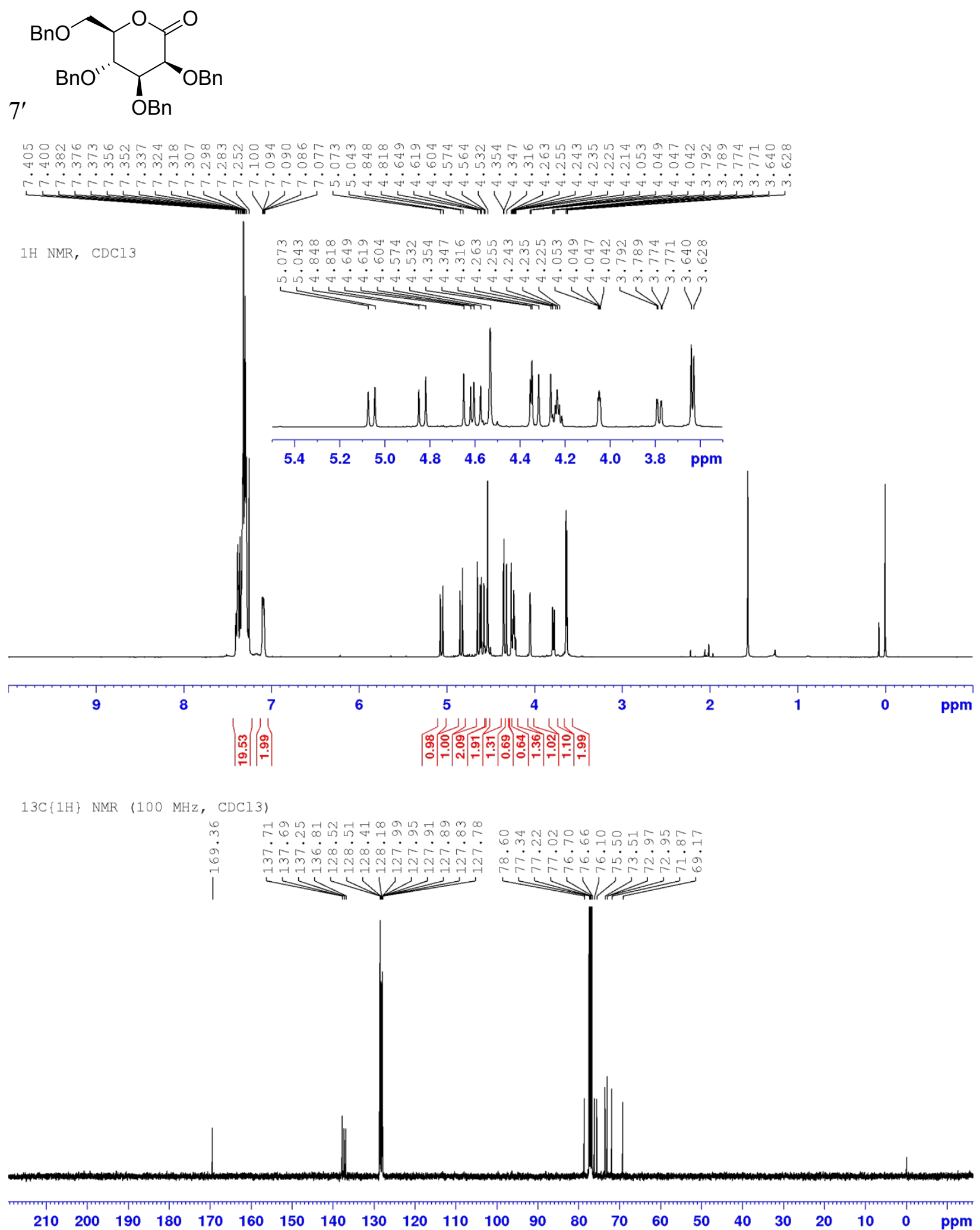


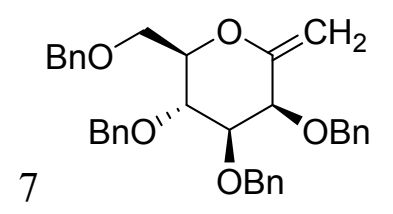

6
0 ن

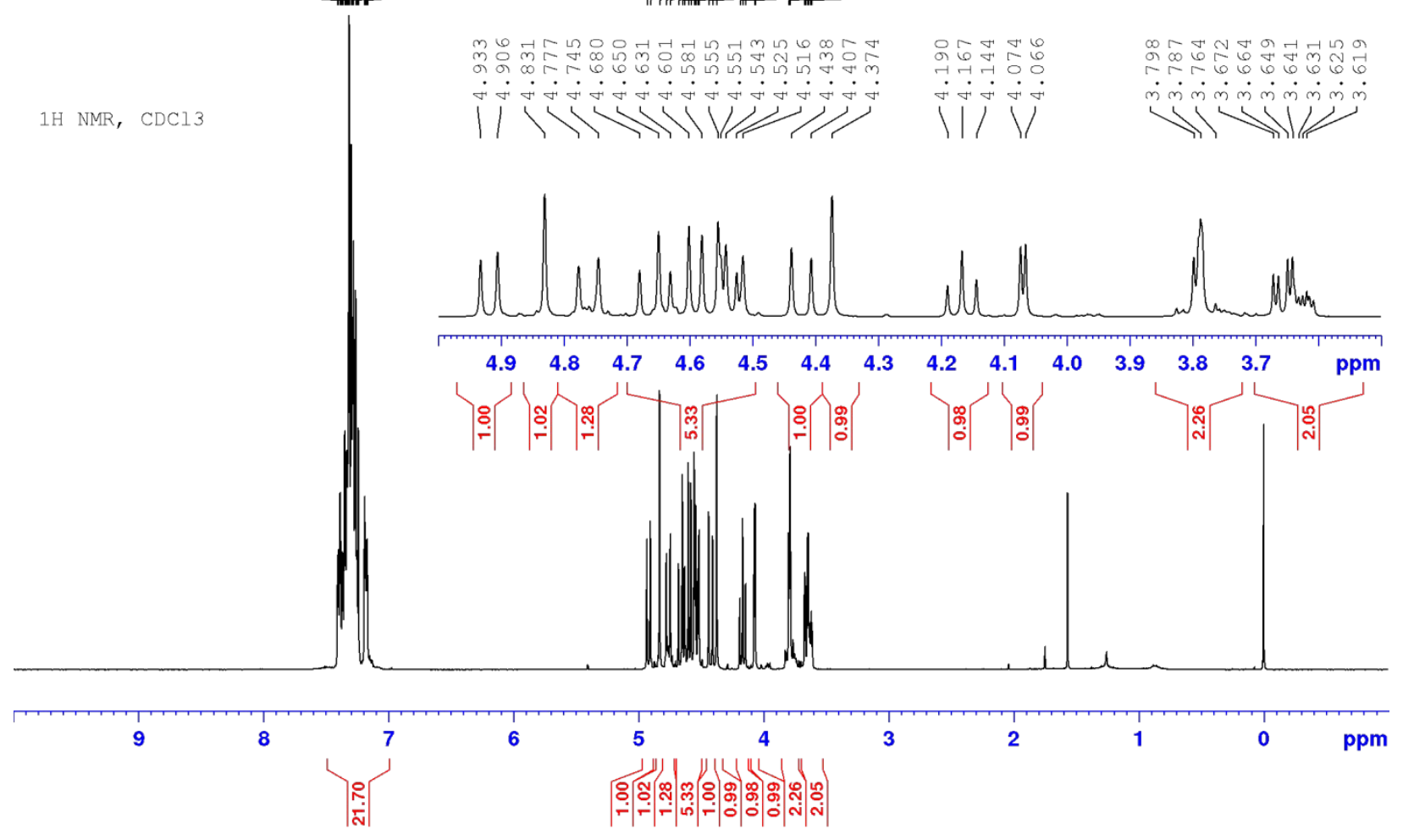




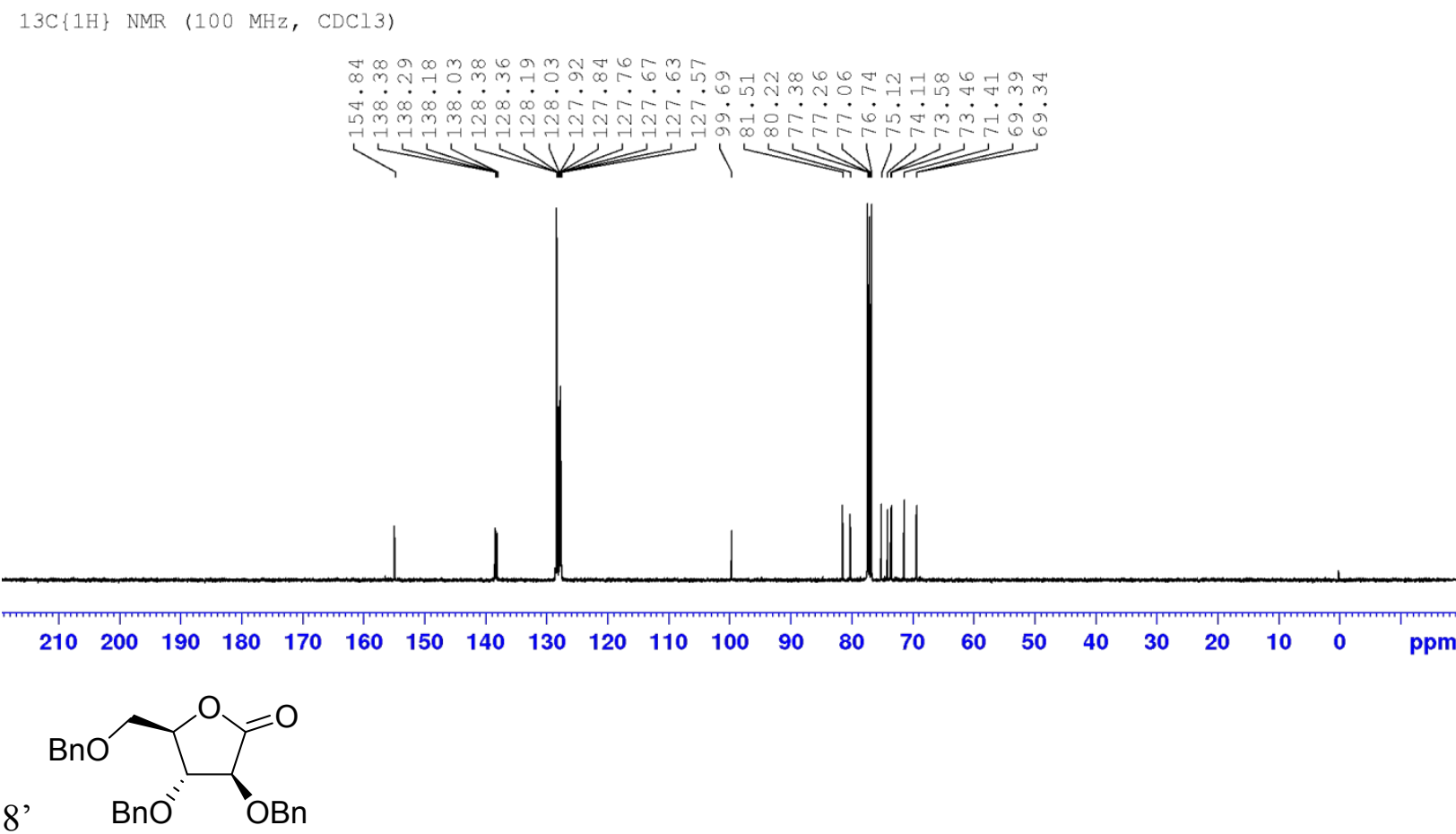

$1 \mathrm{H}$ NMR (400 MHz, CDCl3)

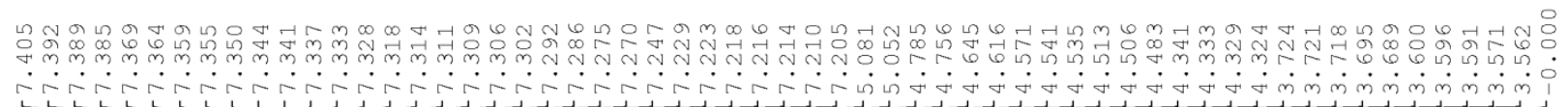

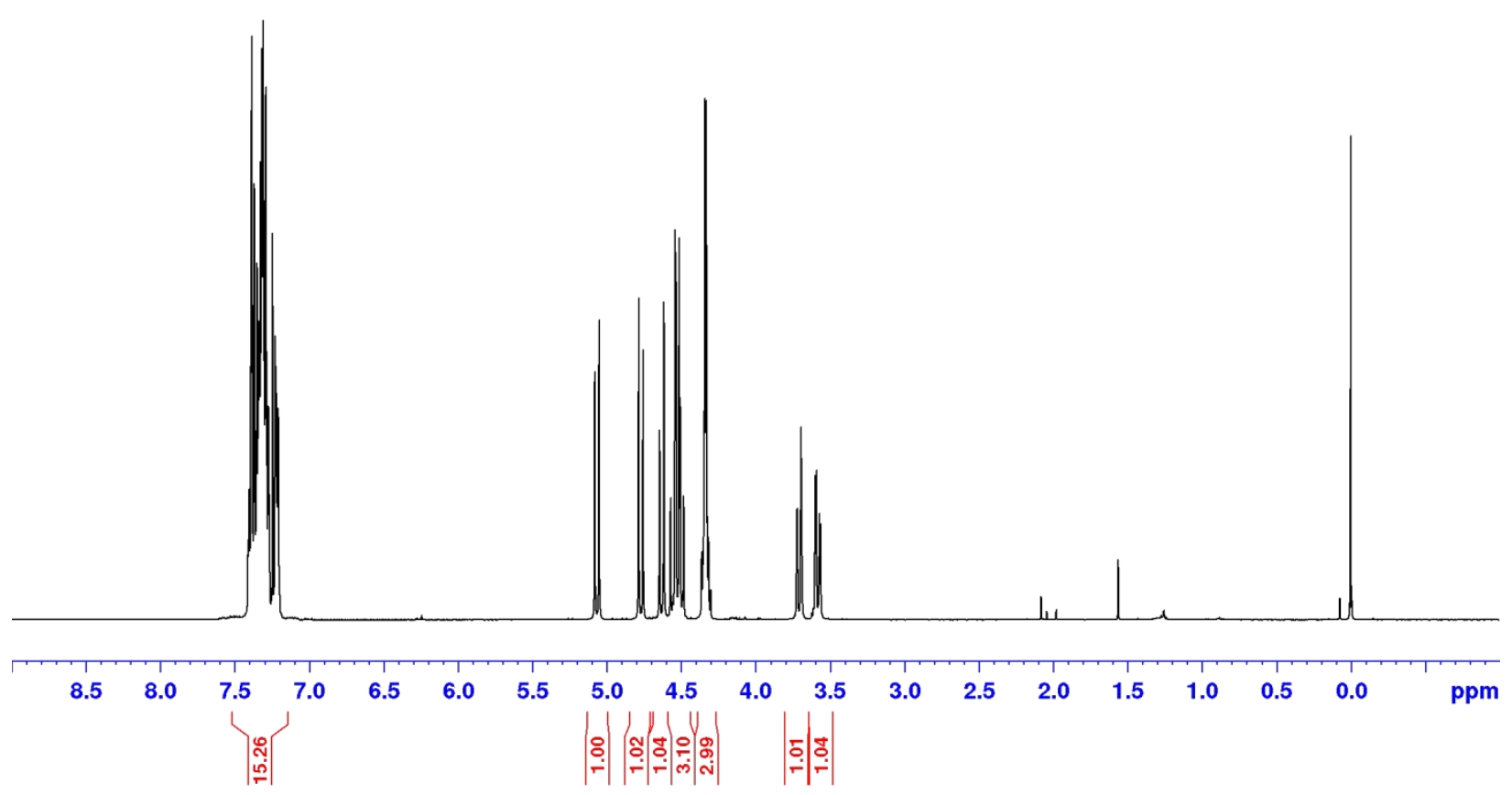



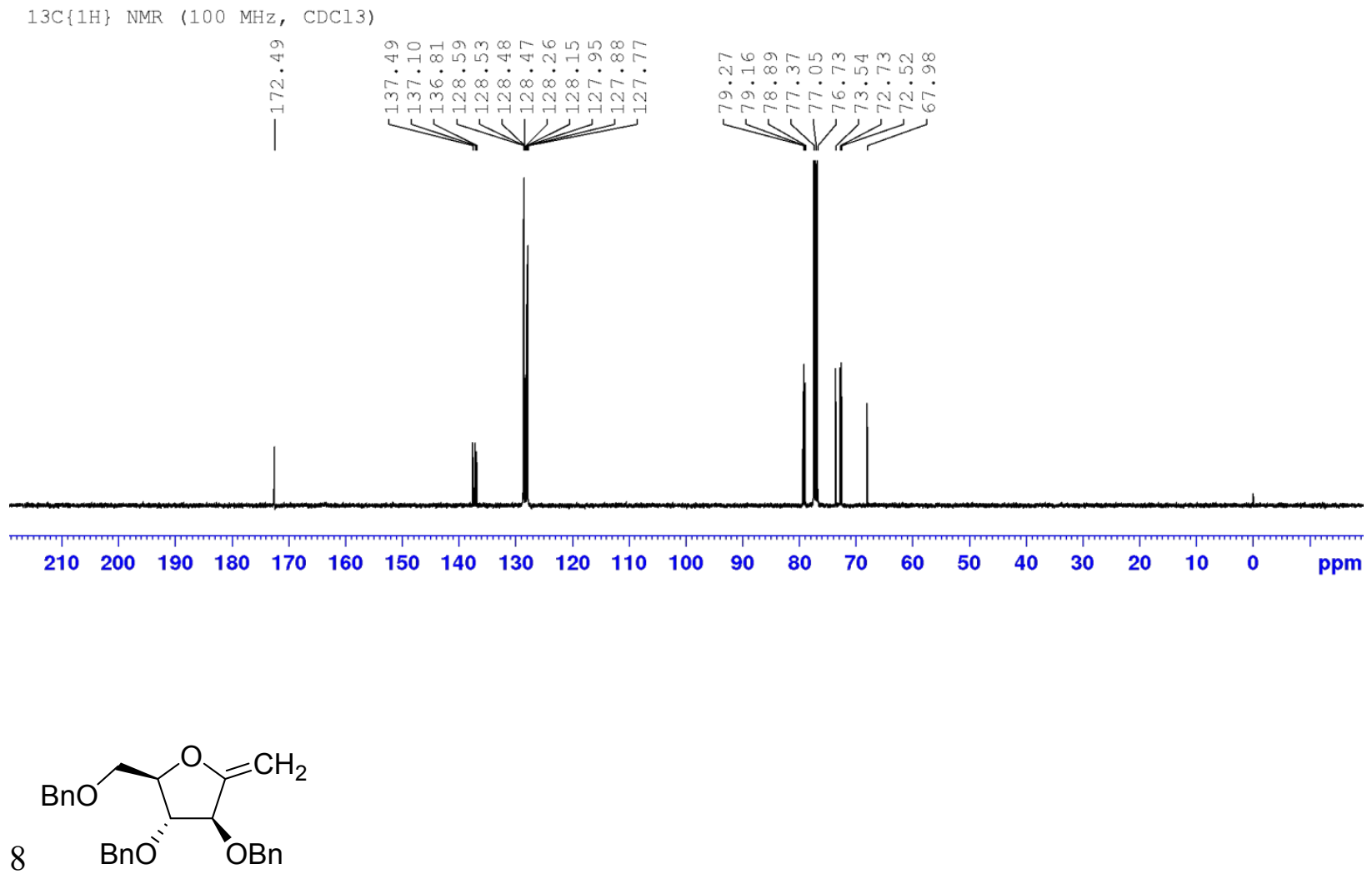


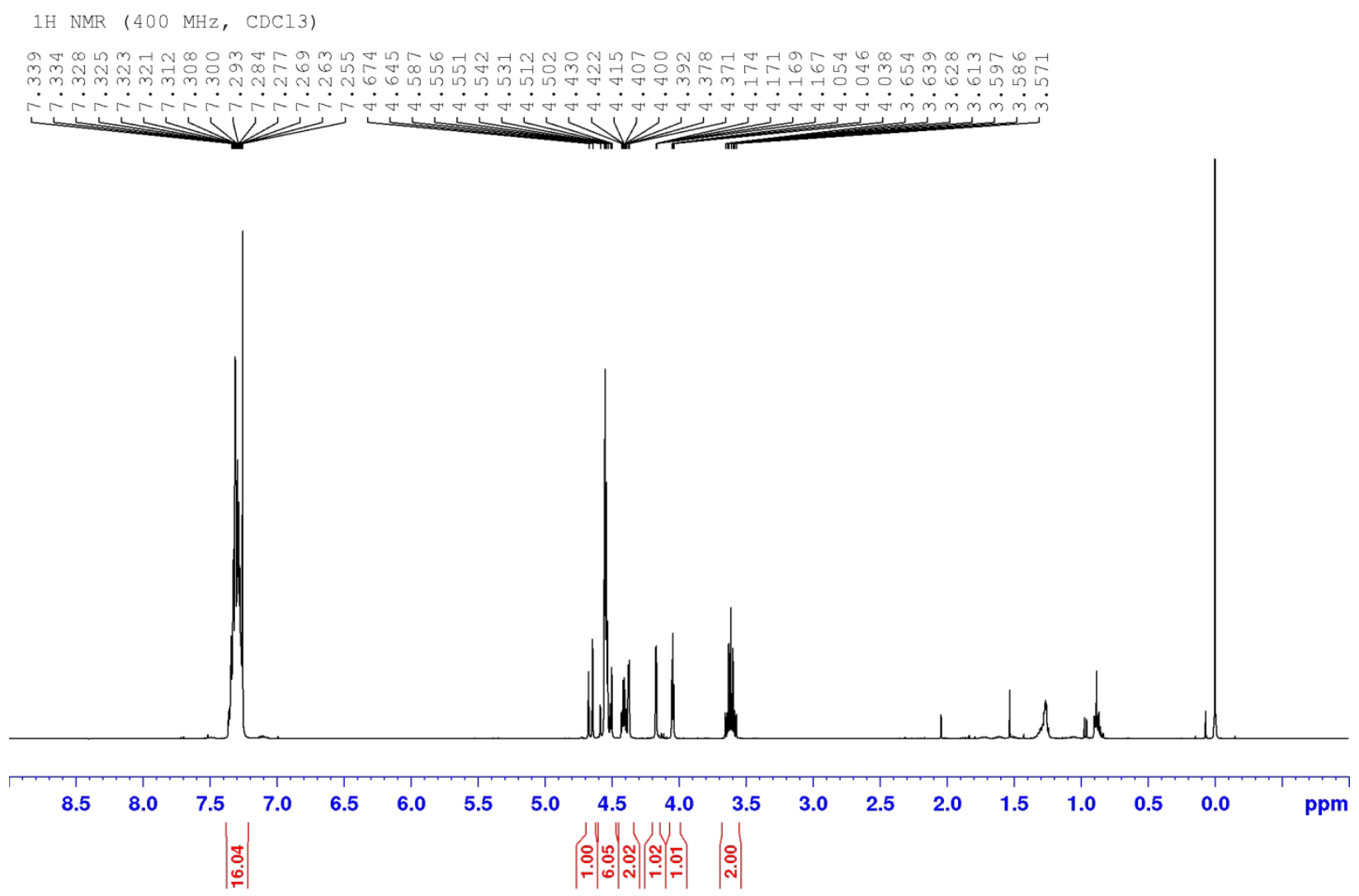

13C $\{1 \mathrm{H}\}$ NMR (100 MHz, CDCl3)

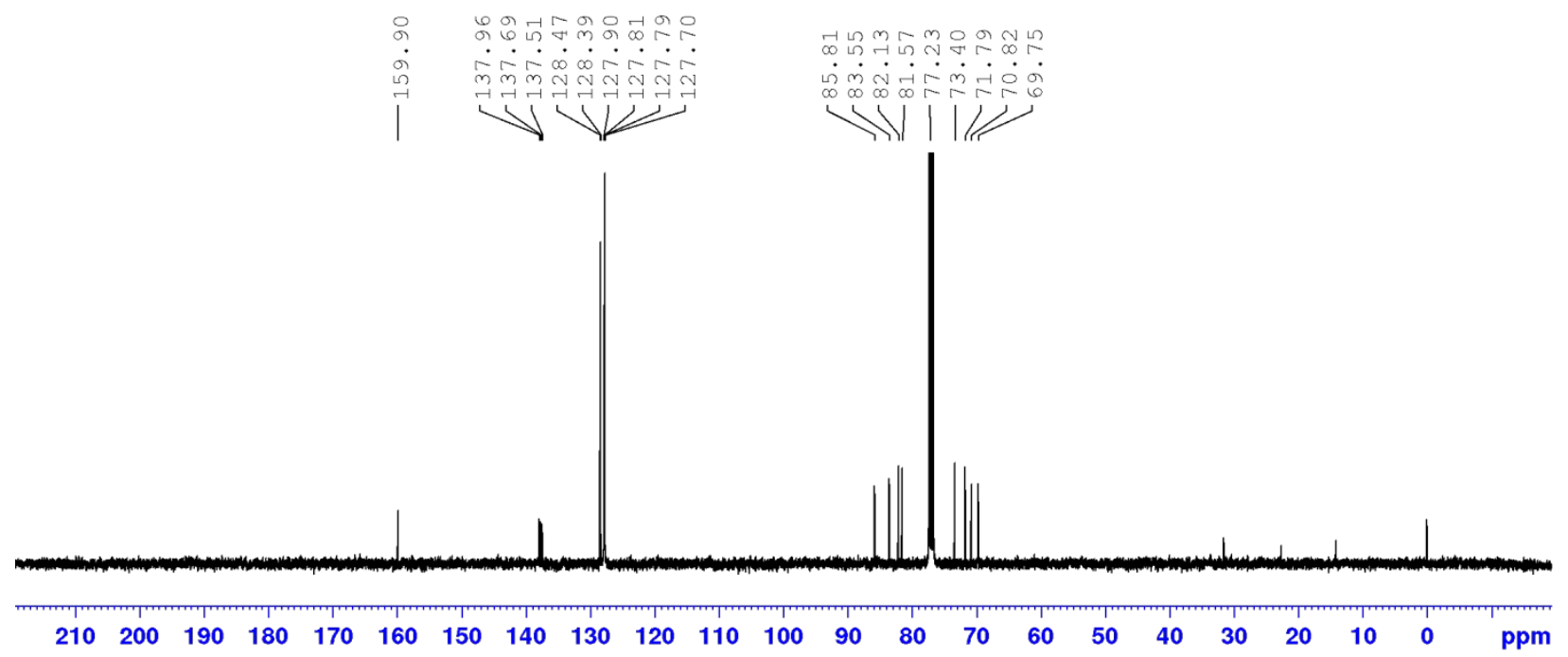




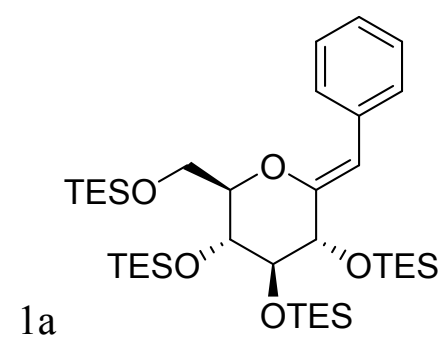

$1 \mathrm{H}$ NMR $(400 \mathrm{MHz}, \mathrm{CDCl} 3)$

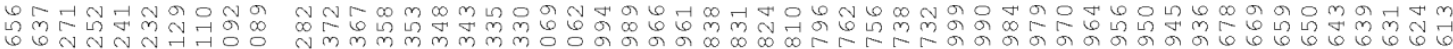

-

$\rightarrow+1$

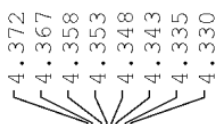

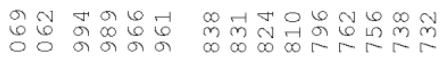

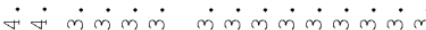

1111
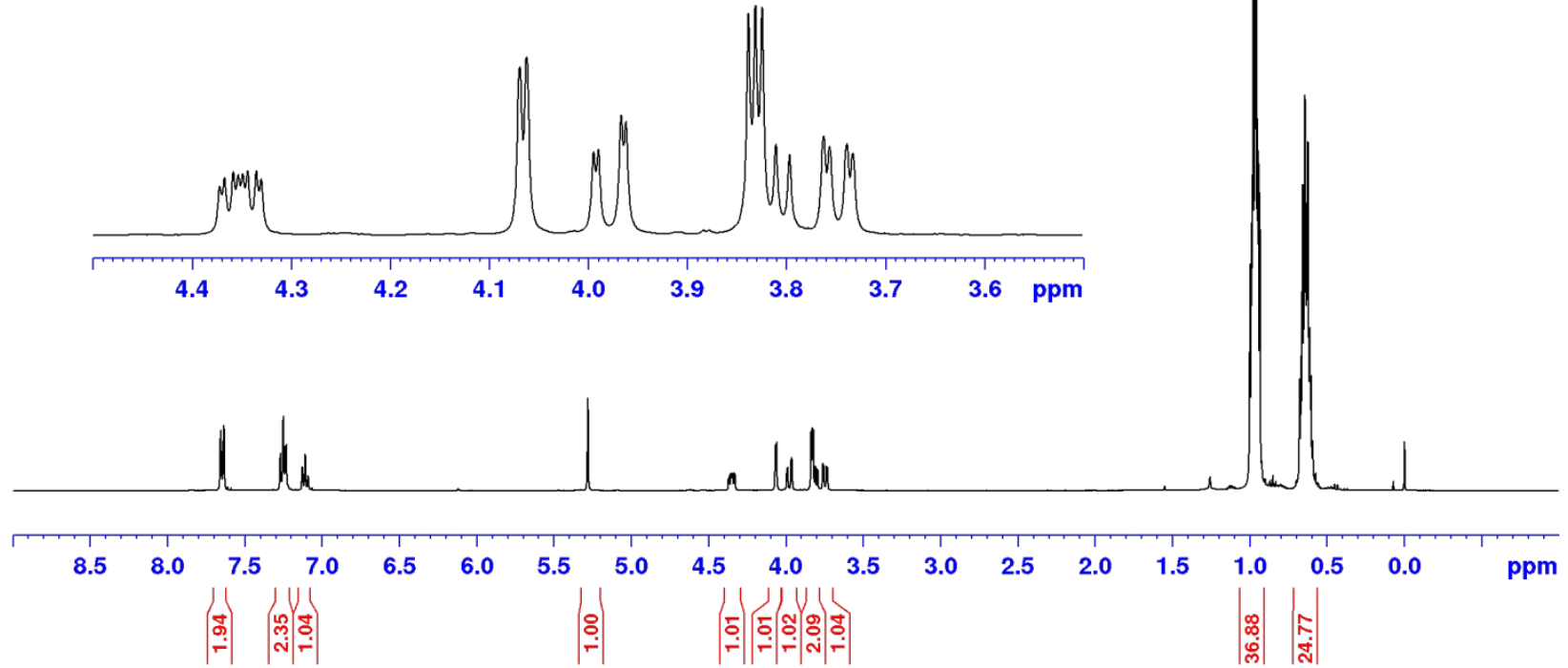


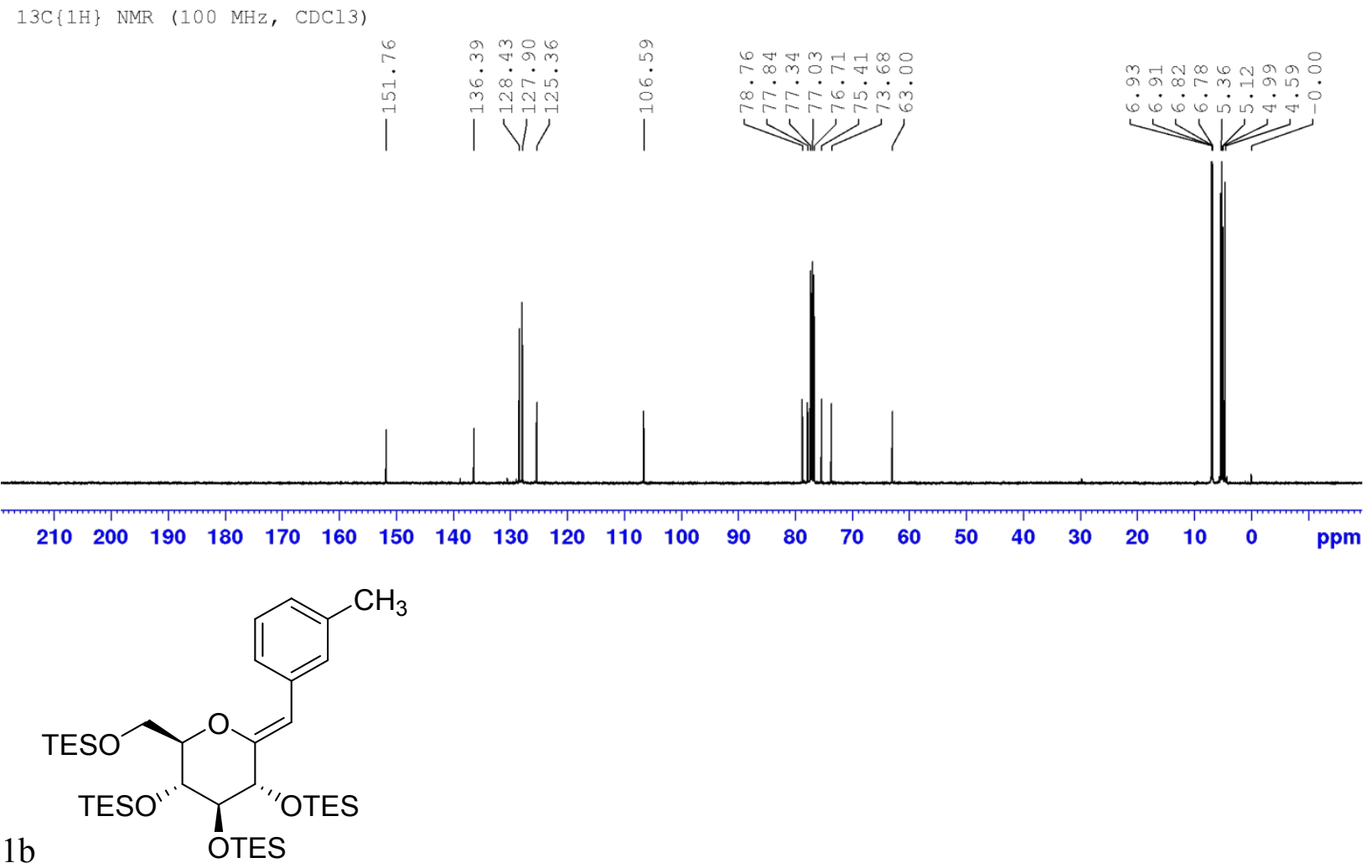




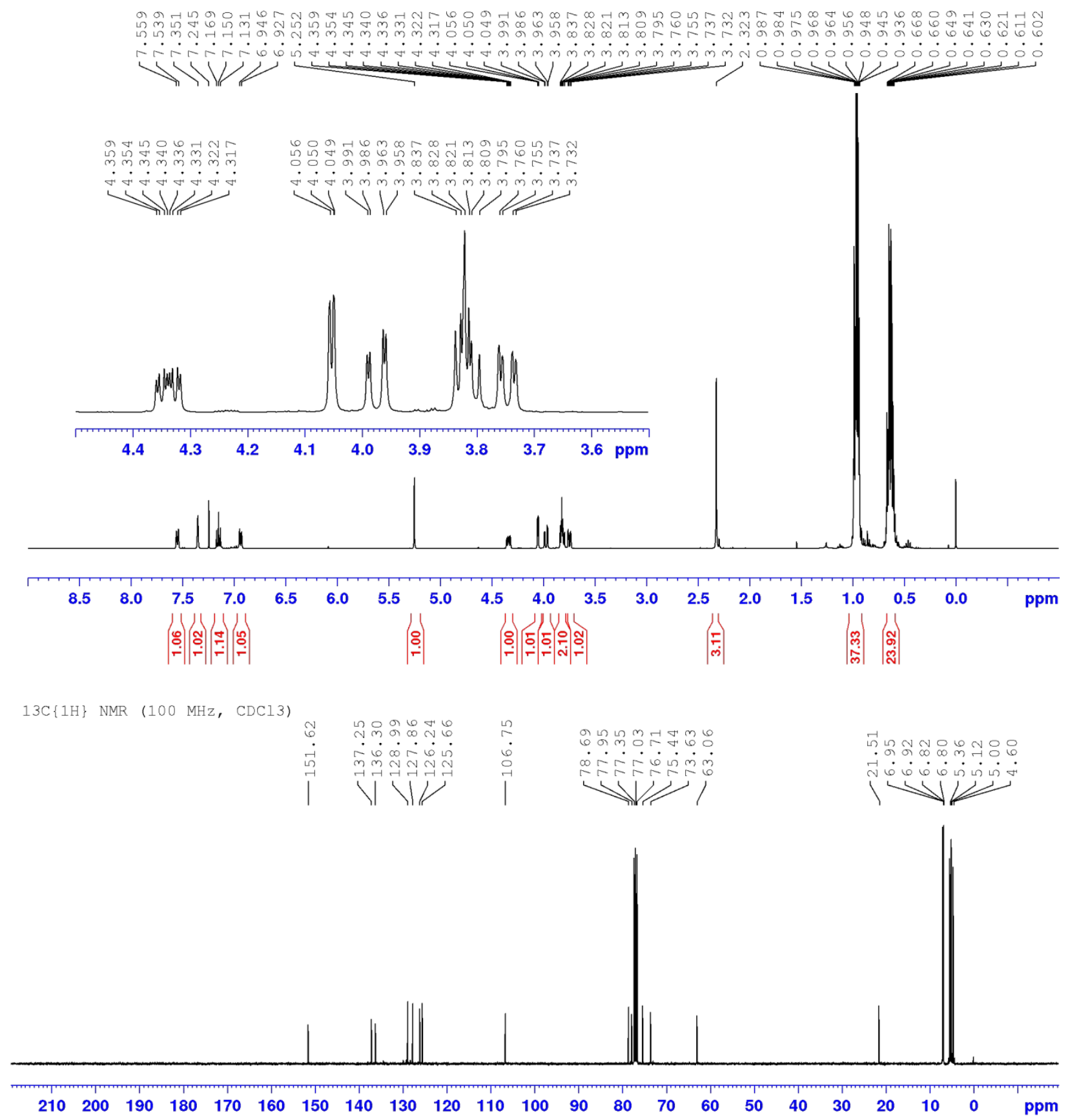




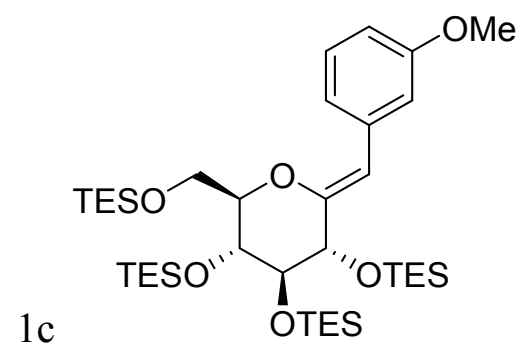
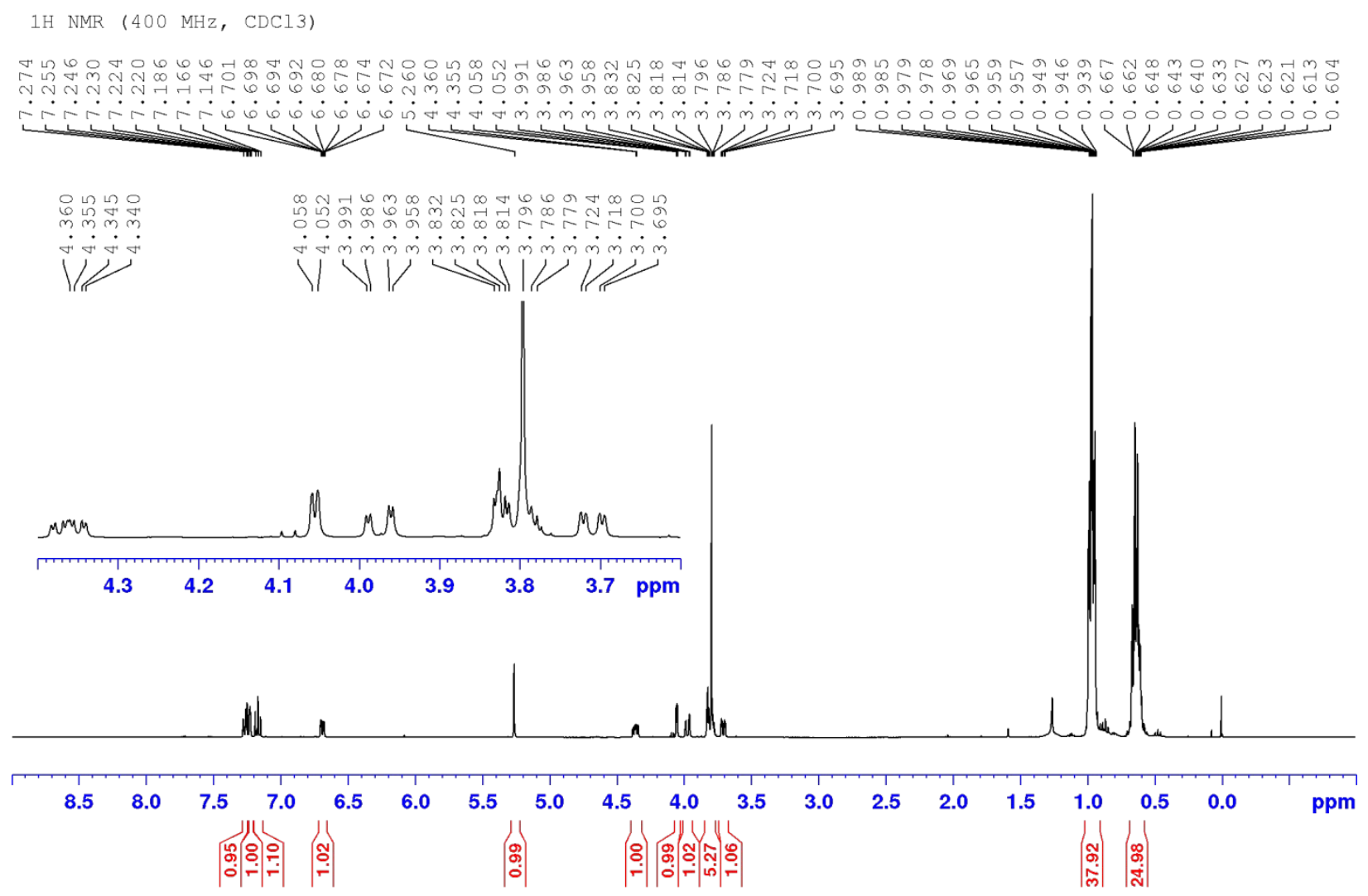


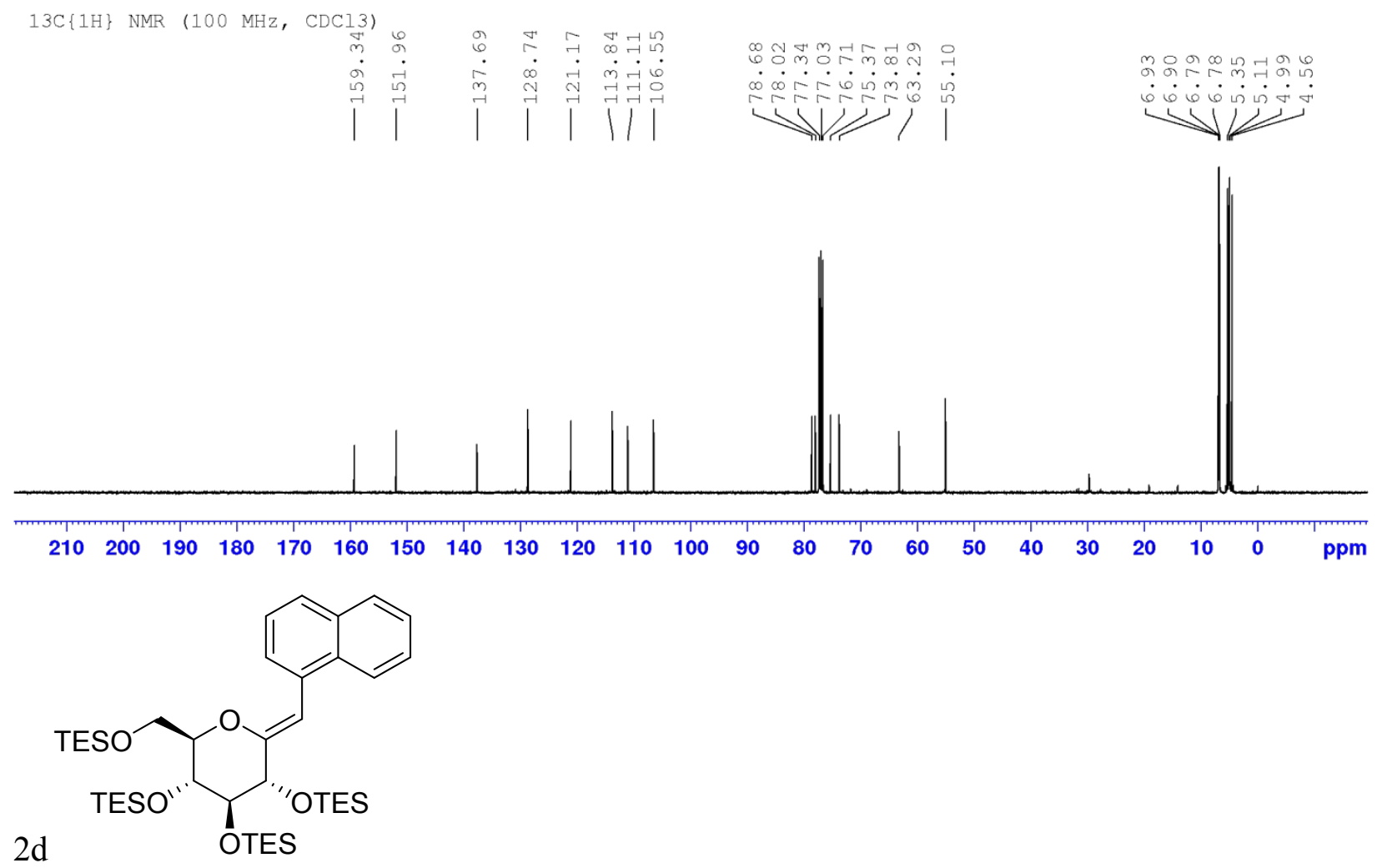



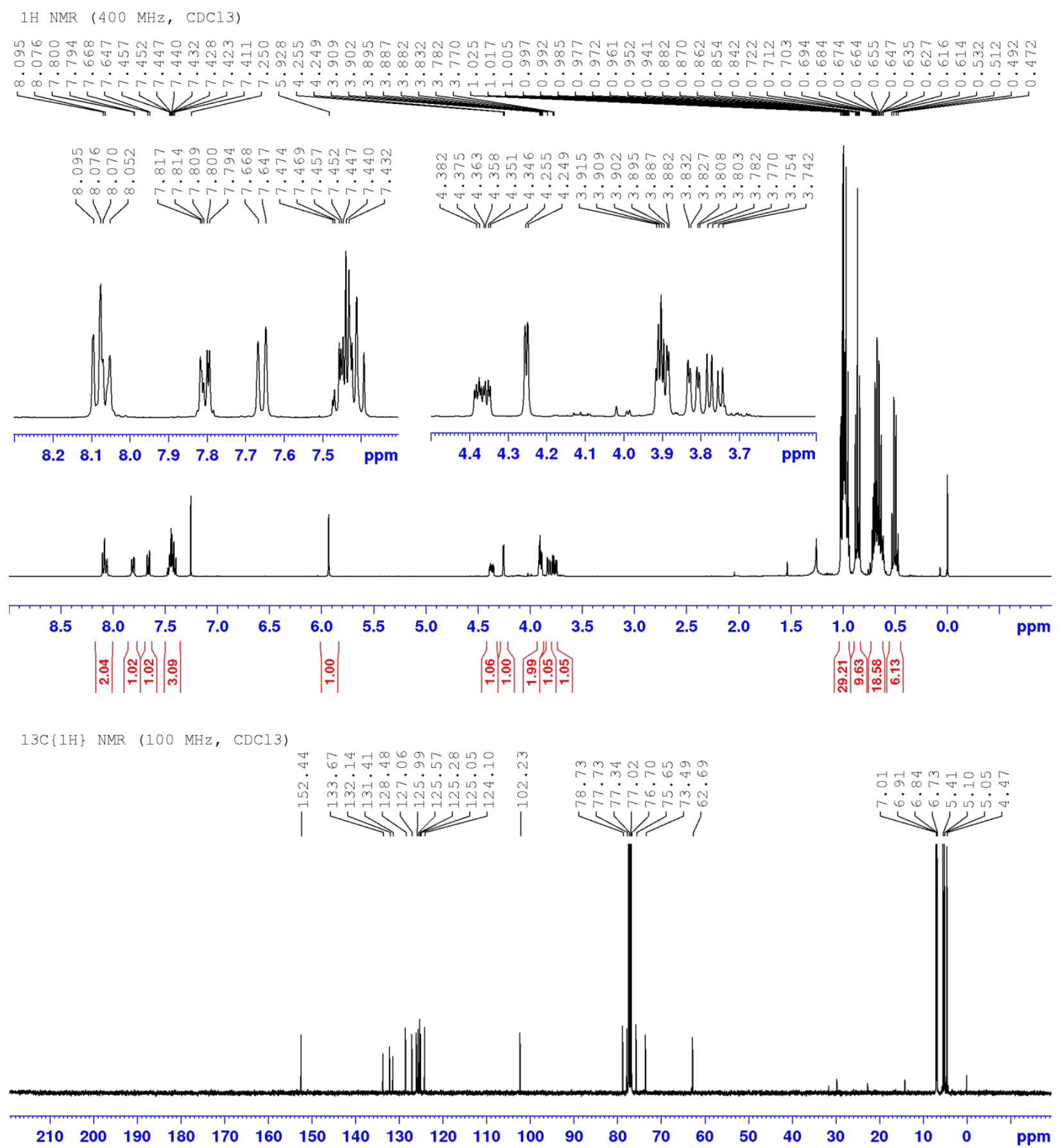
<smiles>CO[C@H]1/C(=C/c2ccc(-c3ccccc3)cc2)O[C@H](CO[SH](=O)=S)[C@H](OC(F)(F)F)C1OC(F)(F)F</smiles>

1H NMR (400 MHz, CDCl3)

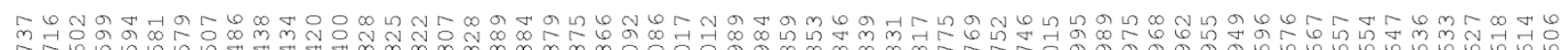

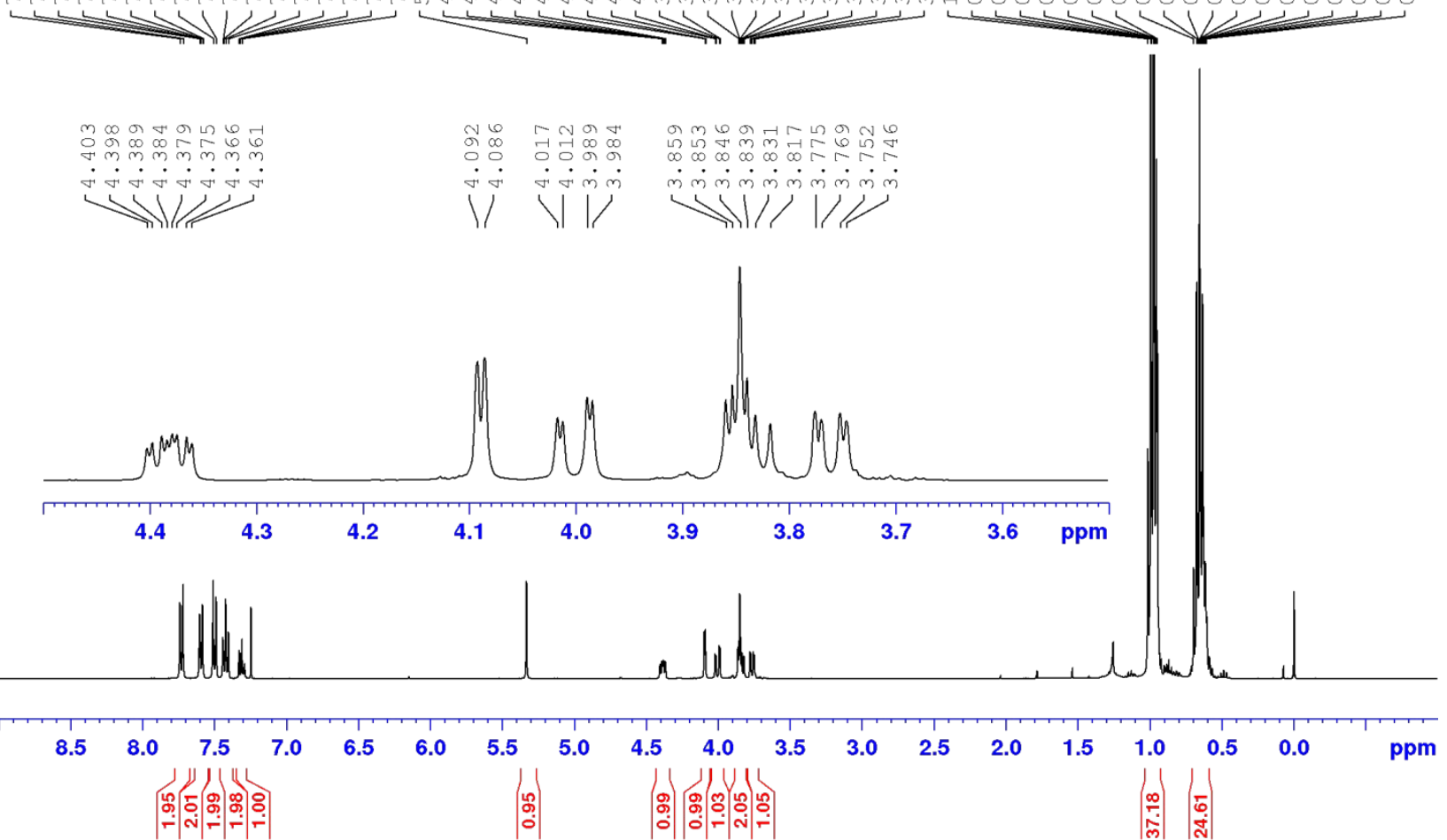




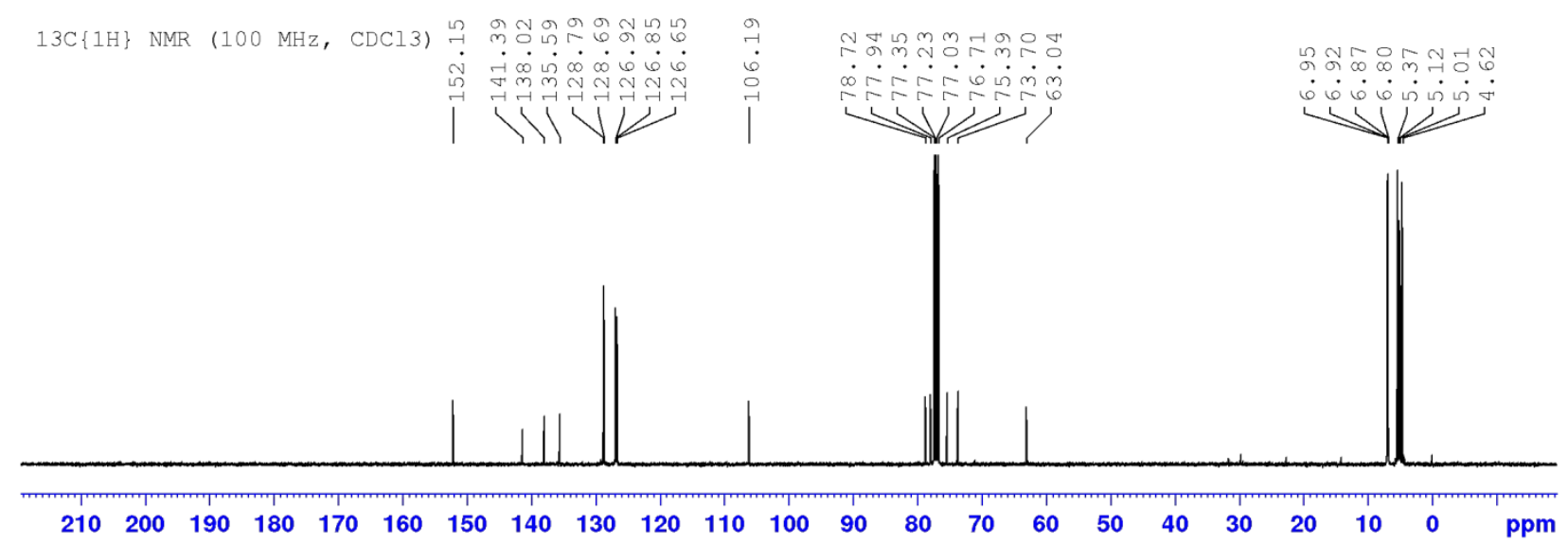

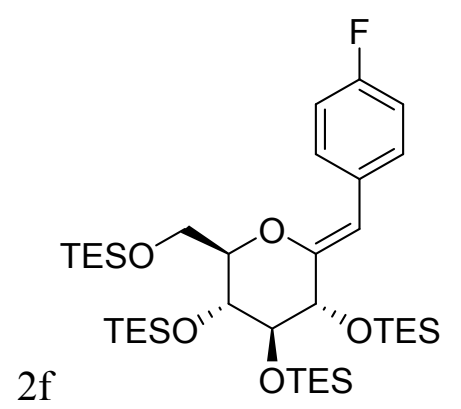

1H NMR (400 MHz, CDCl3)

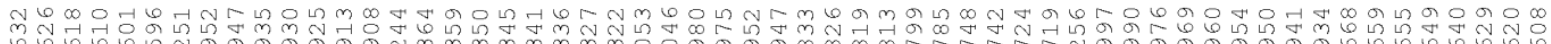

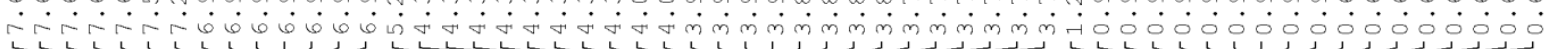

$\longrightarrow+N$

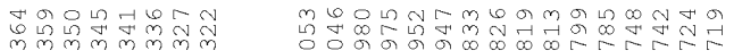

-

$+1 / 11$

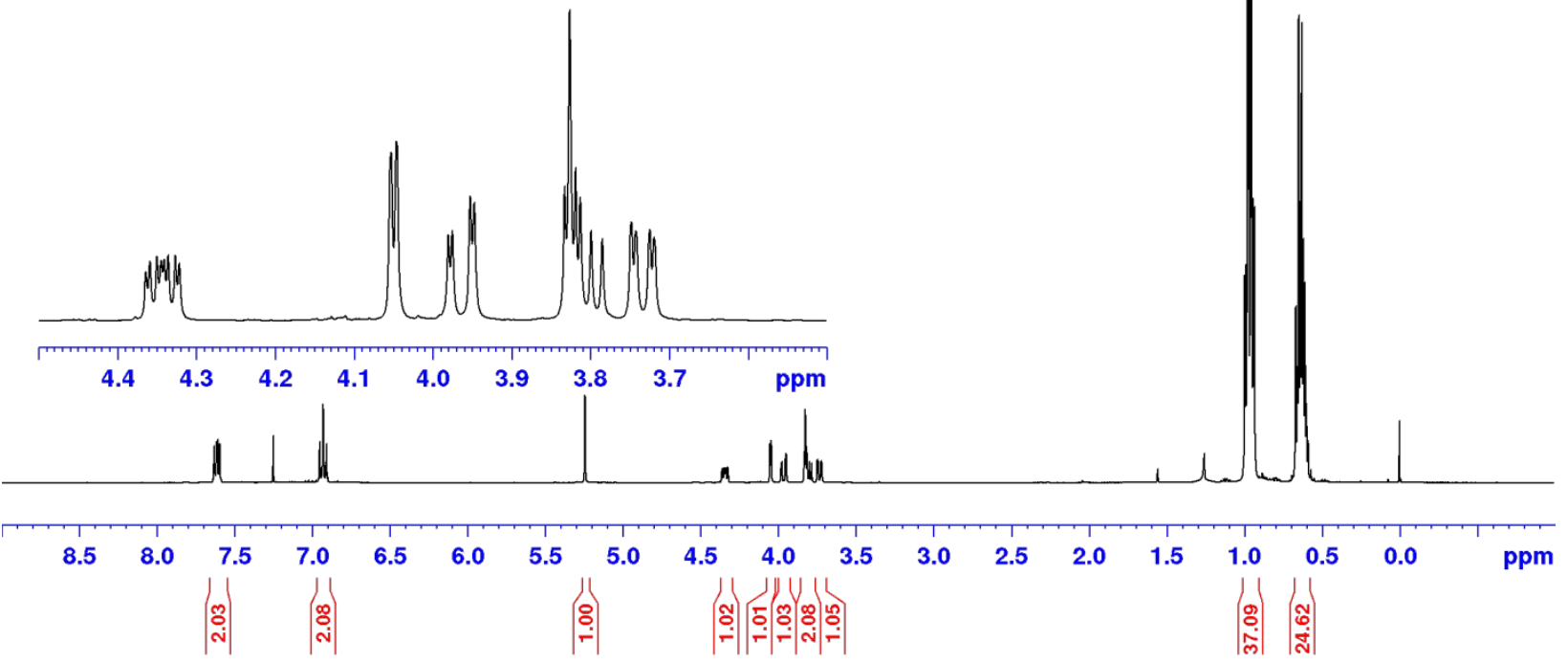




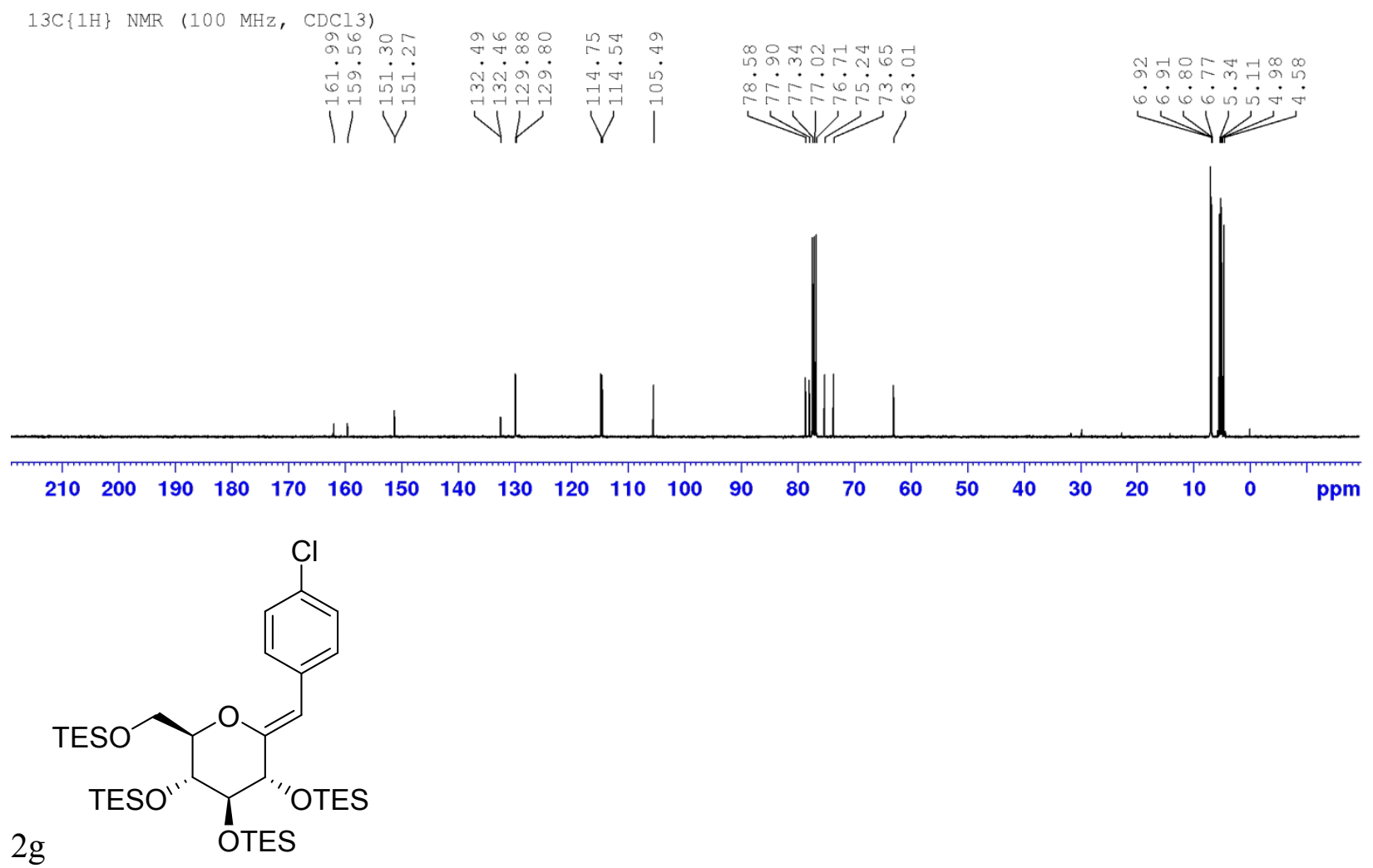


$1 \mathrm{H}$ NMR (400 MHz, CDCl3)

m

-

$\rightarrow 1$

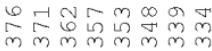

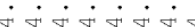

11

मु

$\forall \dot{m} \dot{m} \dot{m} \dot{m} \dot{m} \dot{m} \dot{m} \dot{m}$

$1 / 1 / 111110$
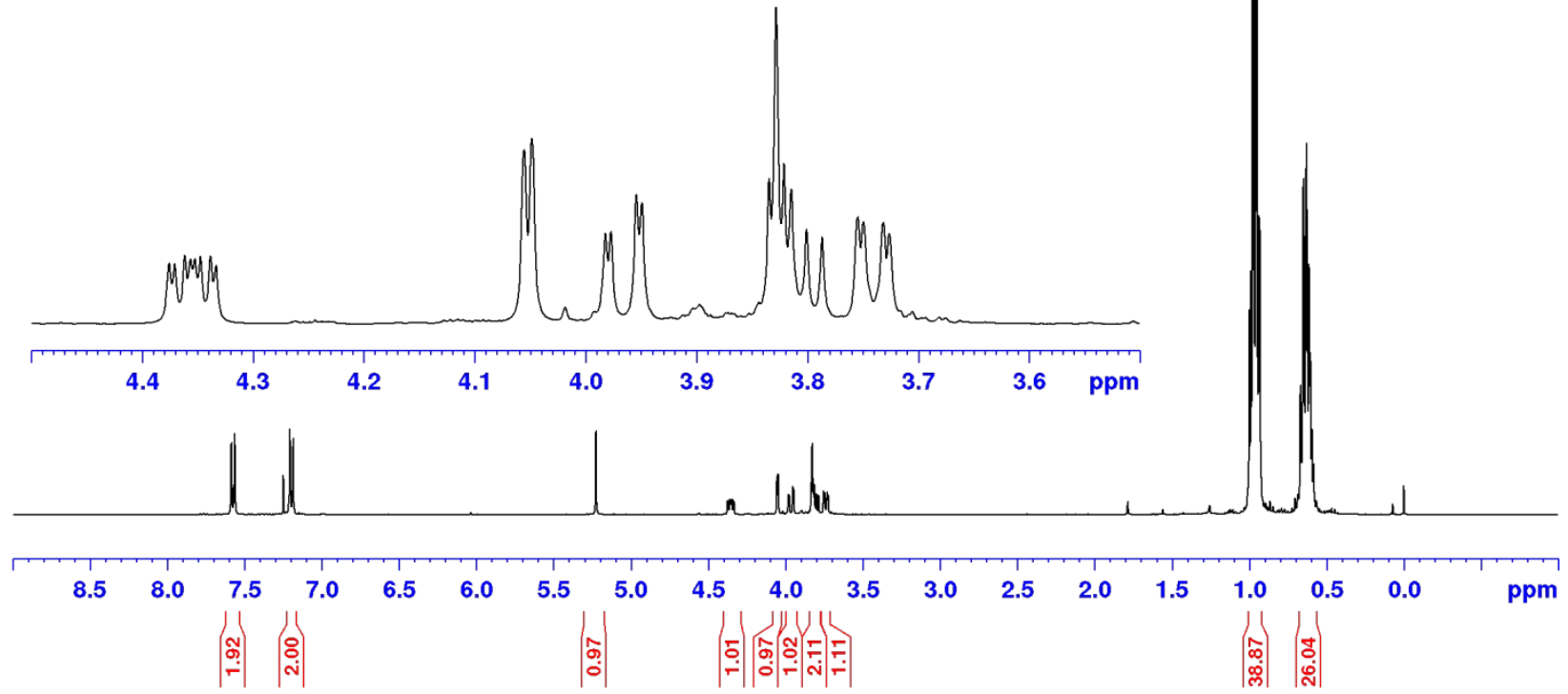

$13 \mathrm{C}\{1 \mathrm{H}\}$ NMR (100 MHz, $\mathrm{CDCl} 3)$
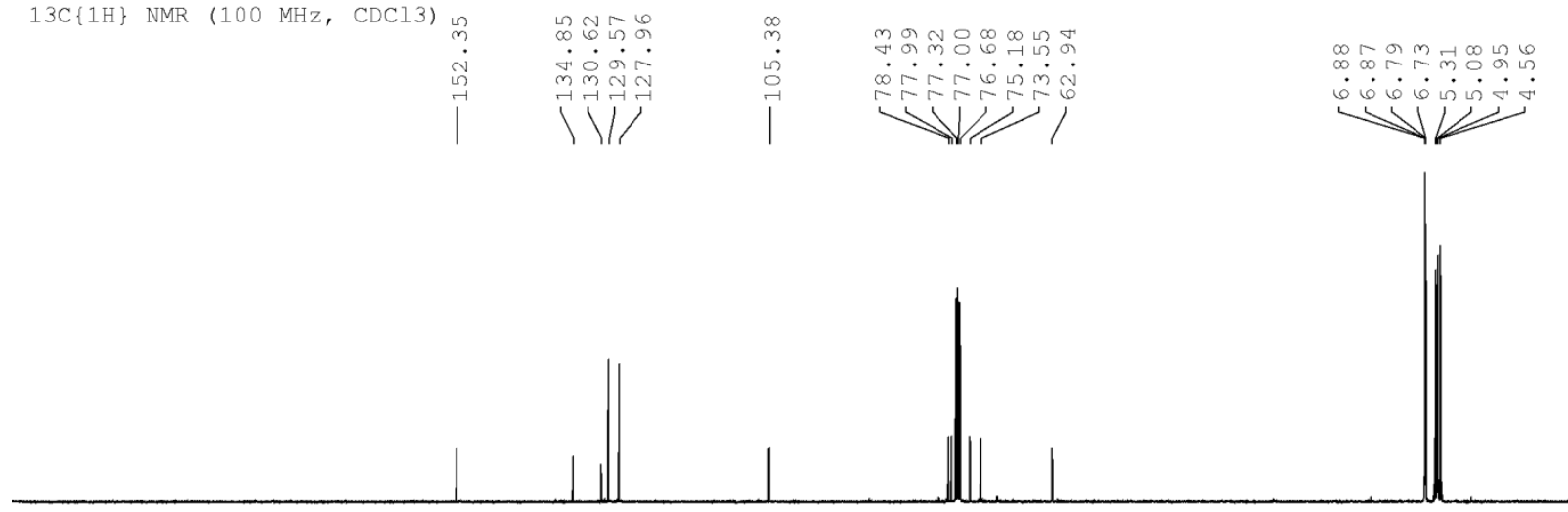

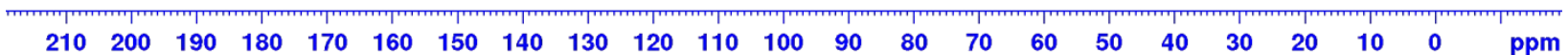




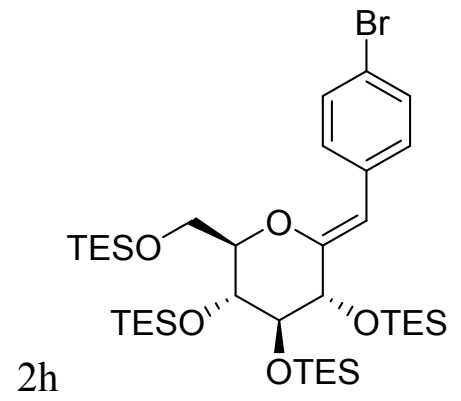
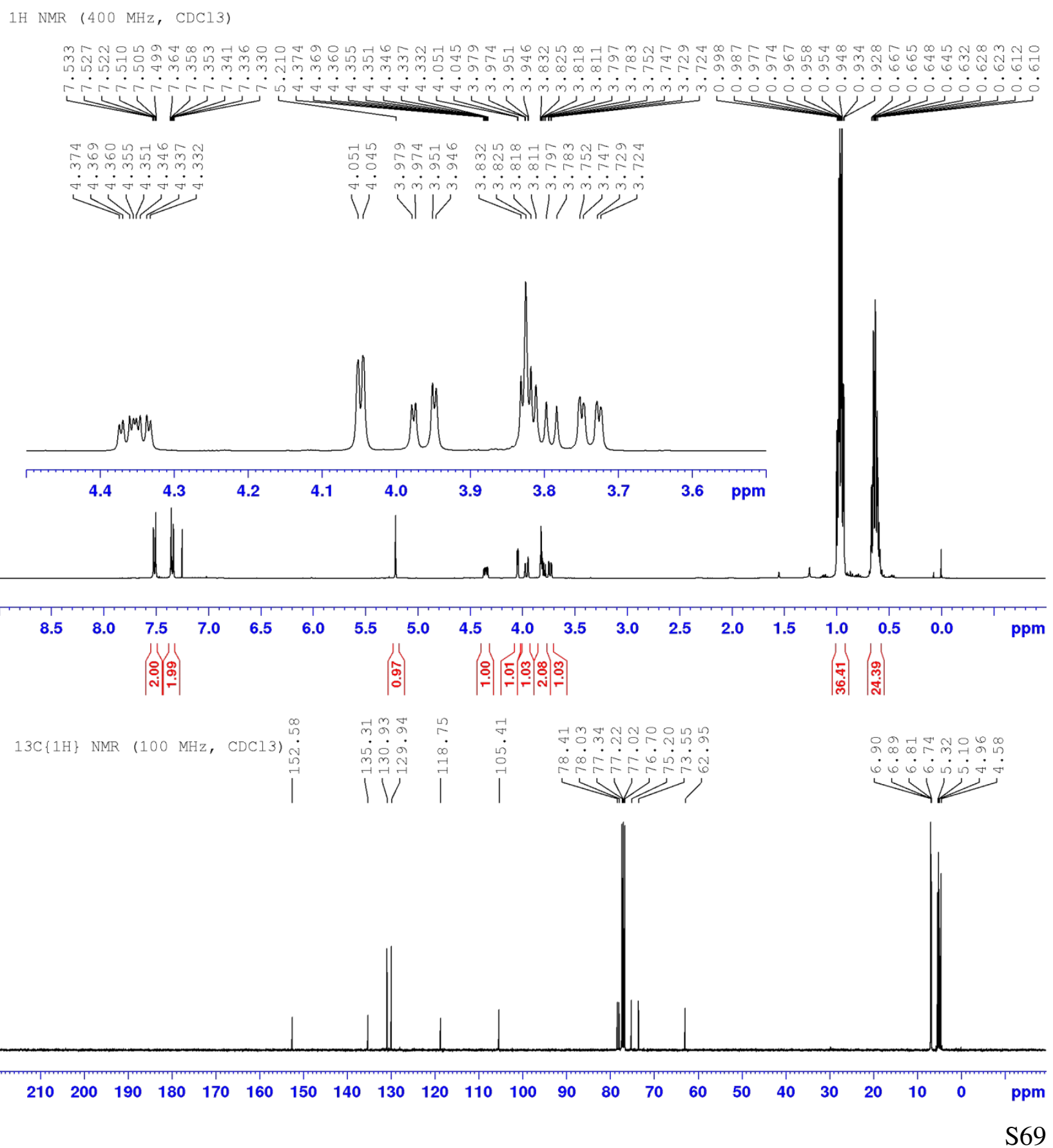


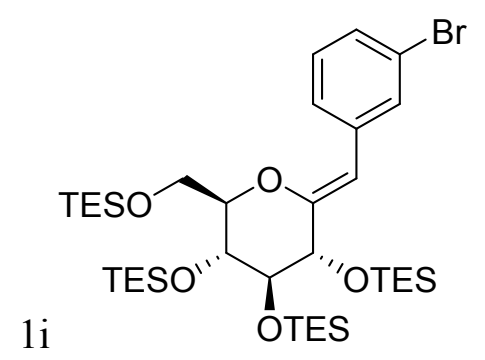

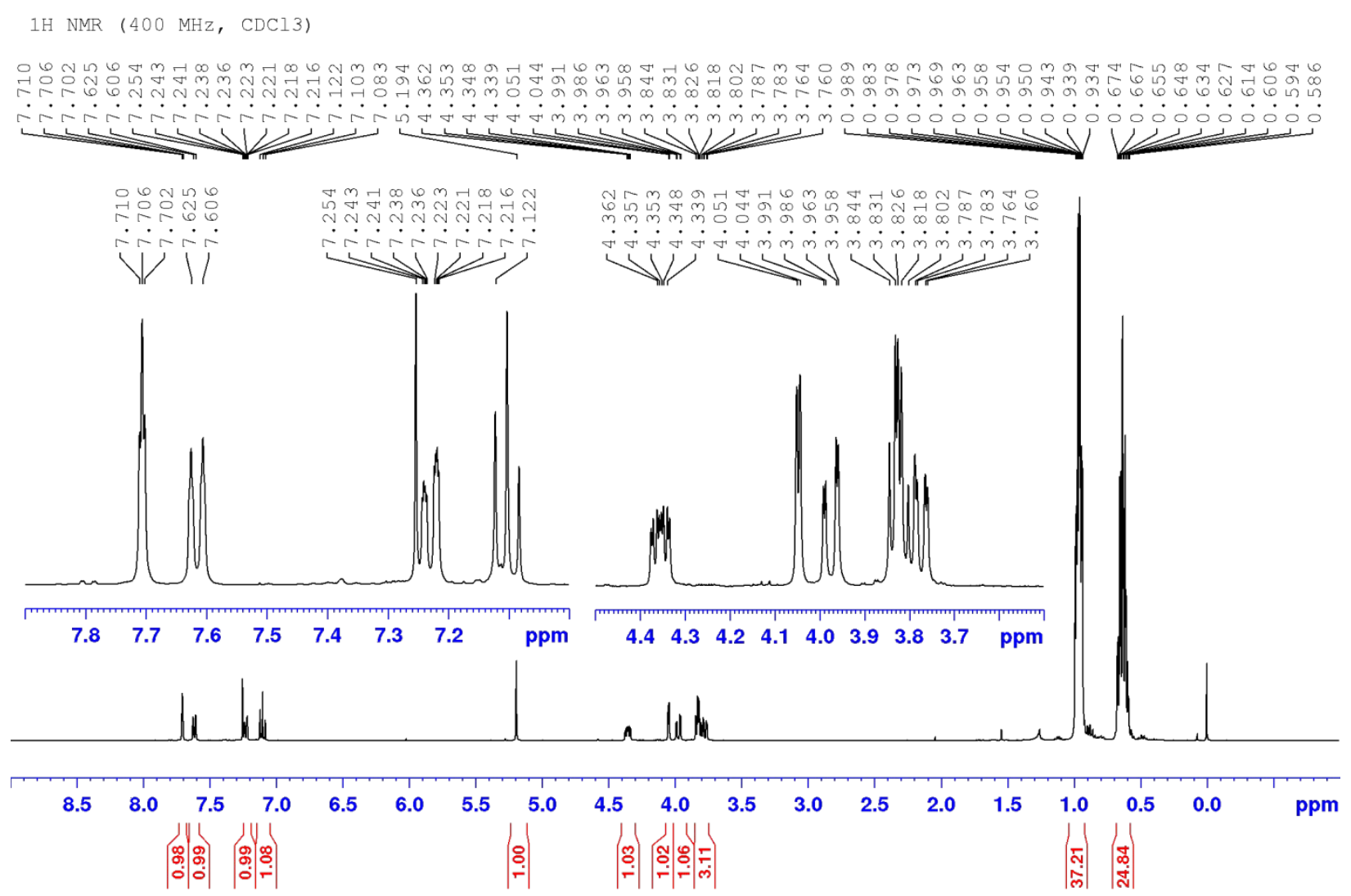




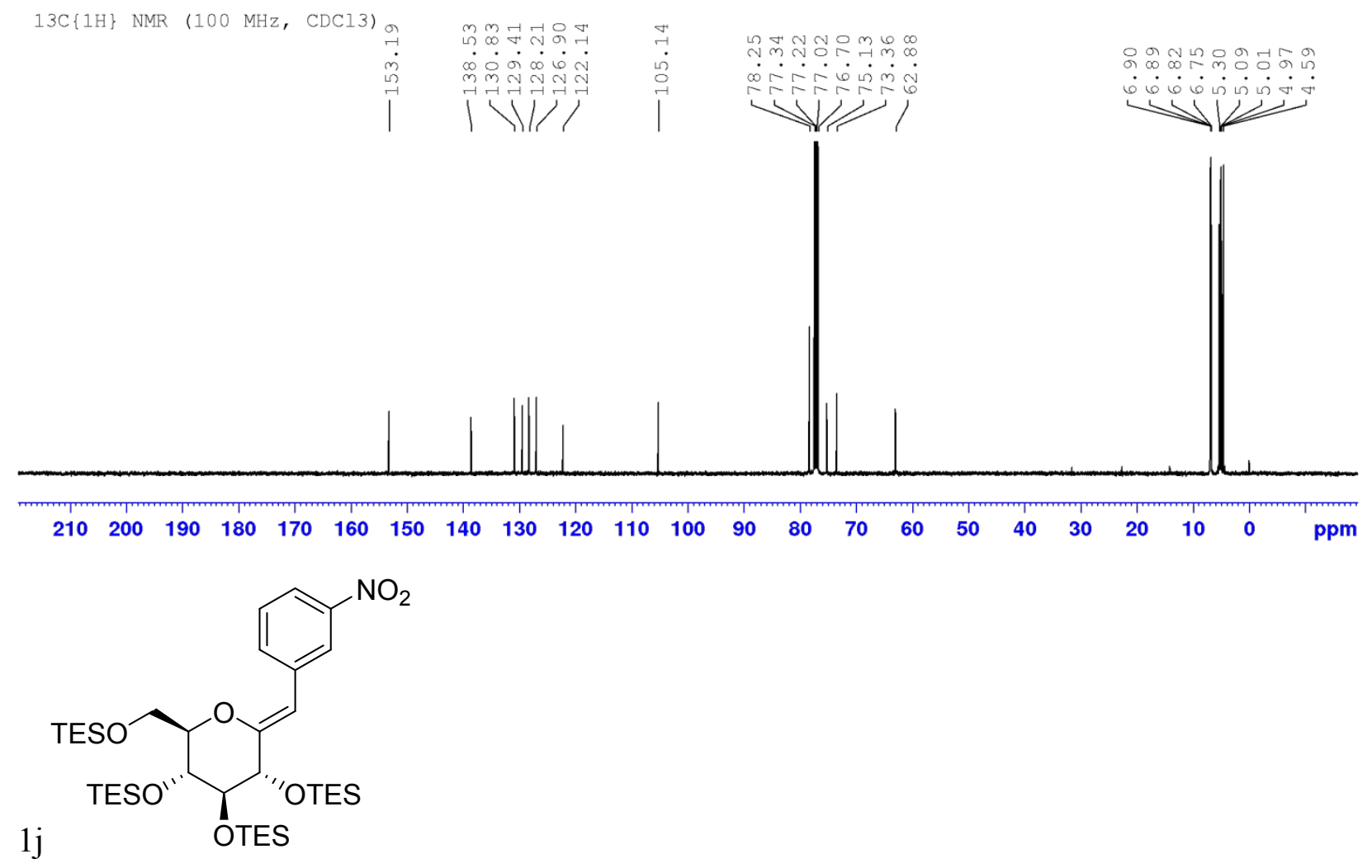




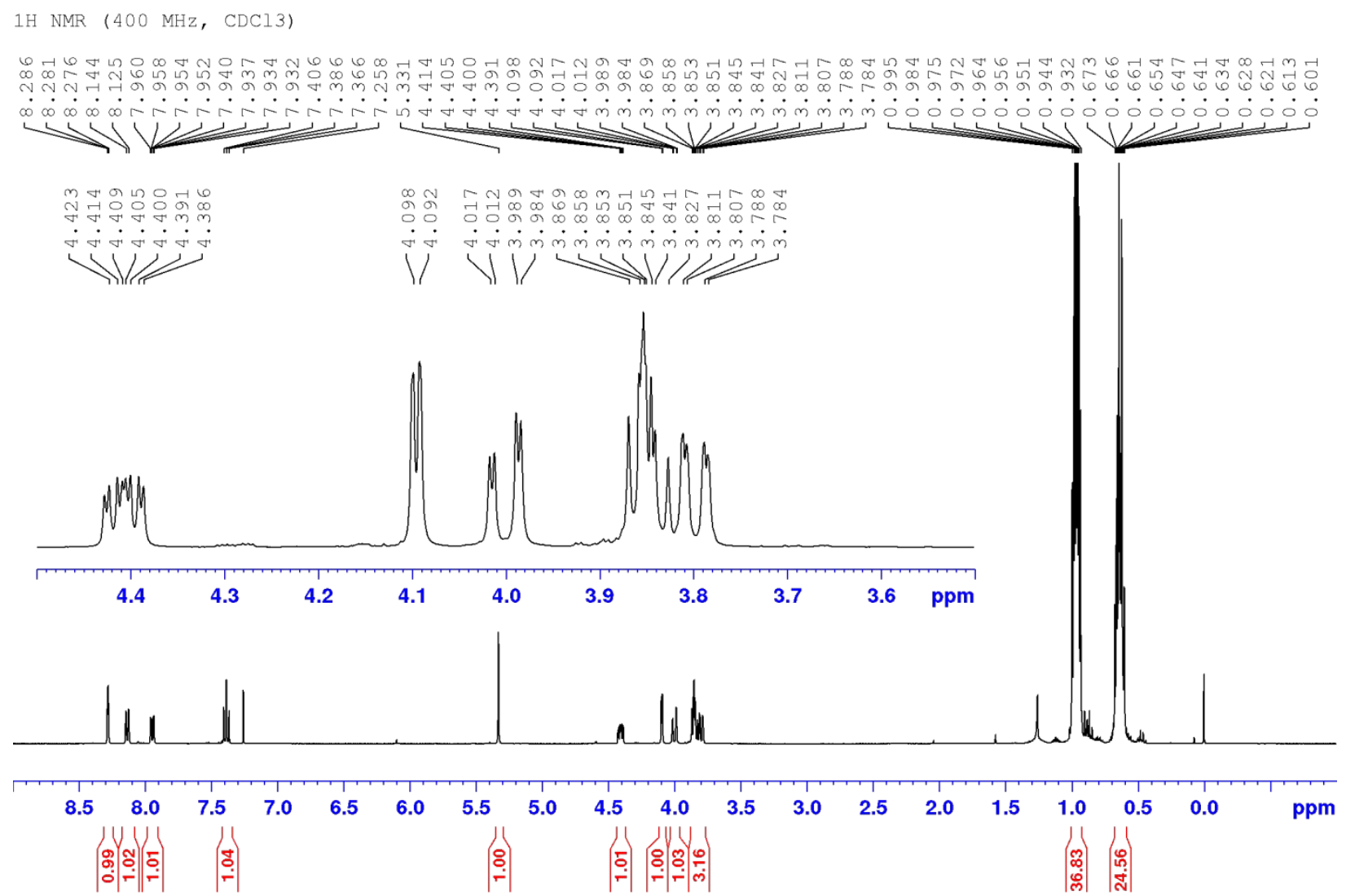

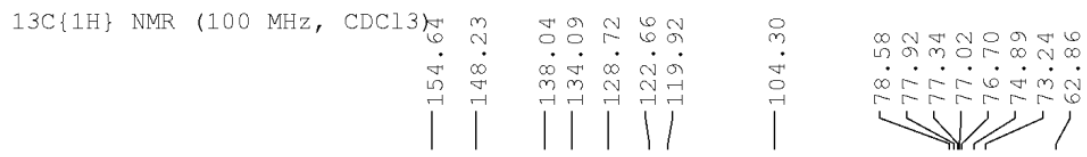
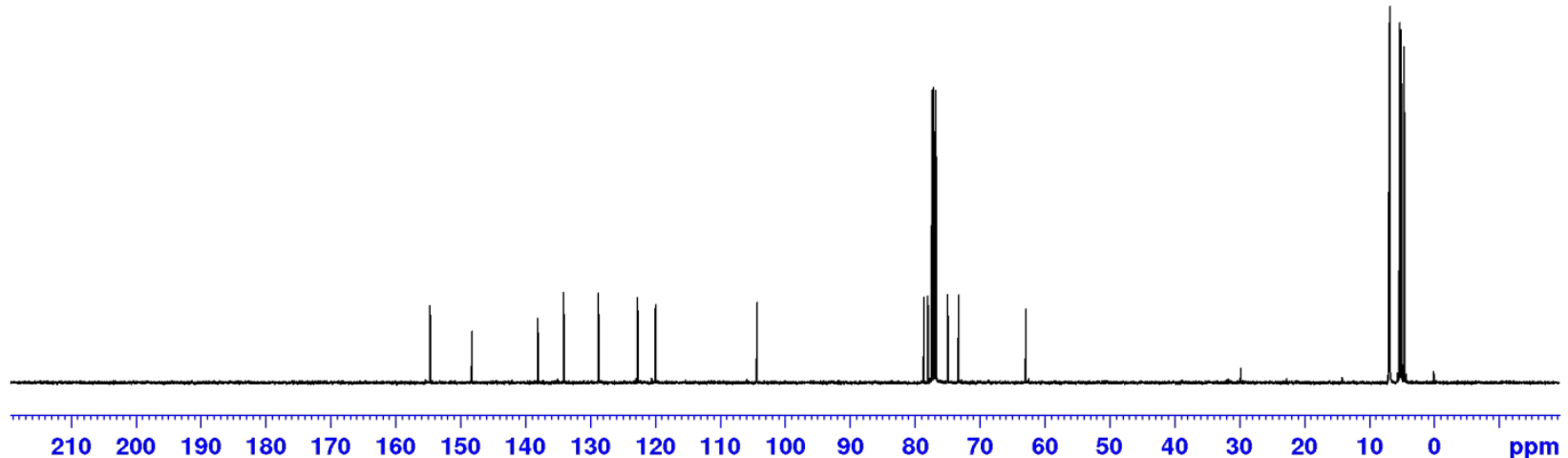


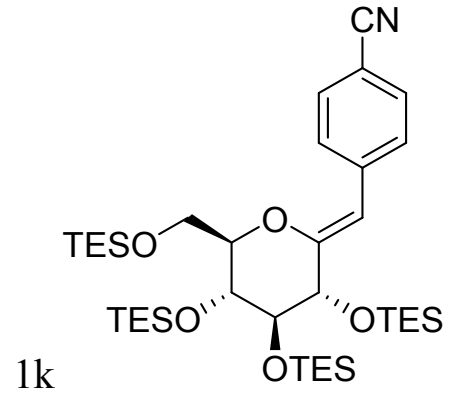

1H NMR (400 MHz, CDCl3)

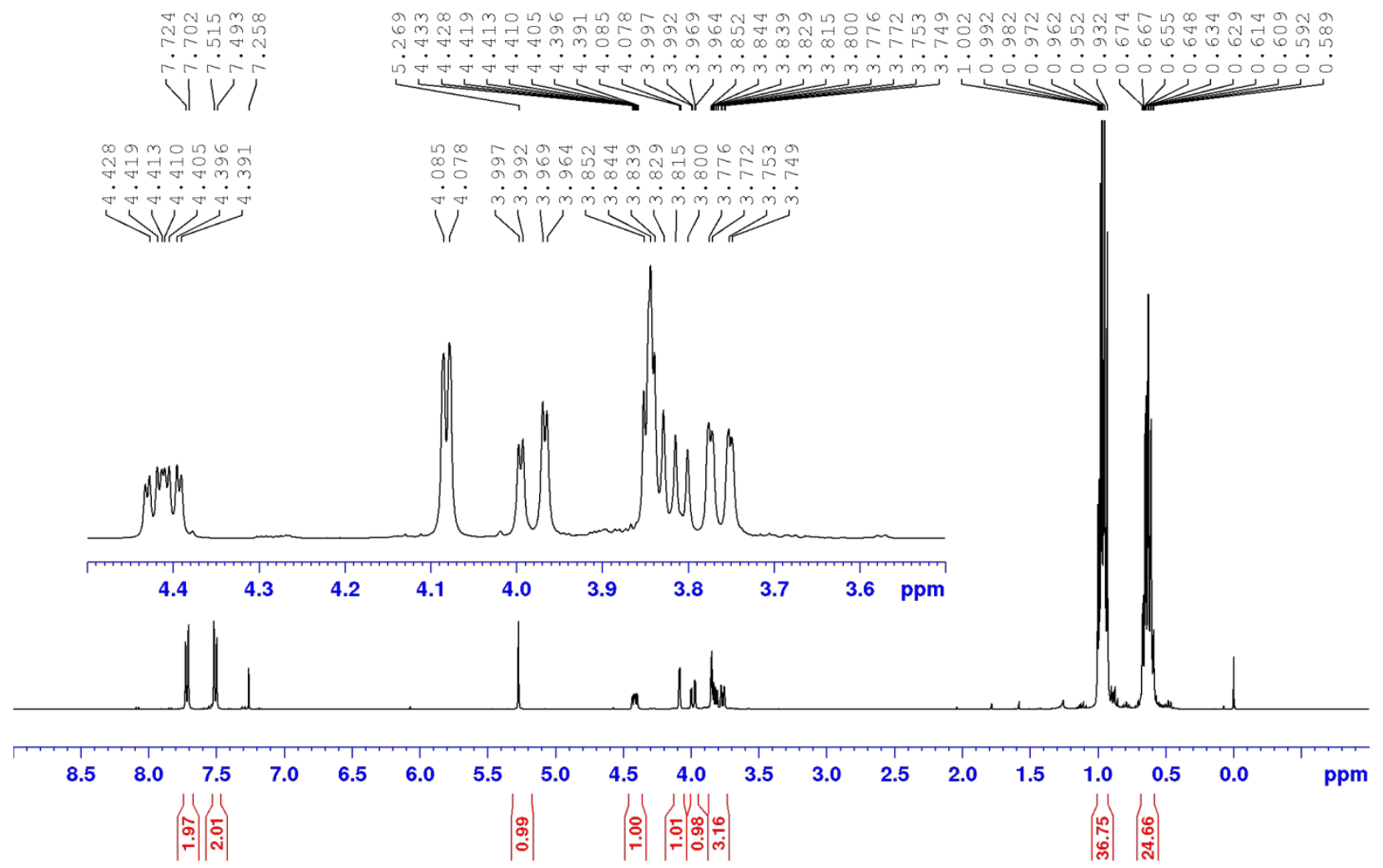

$13 \mathrm{C}\{1 \mathrm{H}\}$ NMR (100 $\mathrm{MHz}, \mathrm{CDCl} 3)$
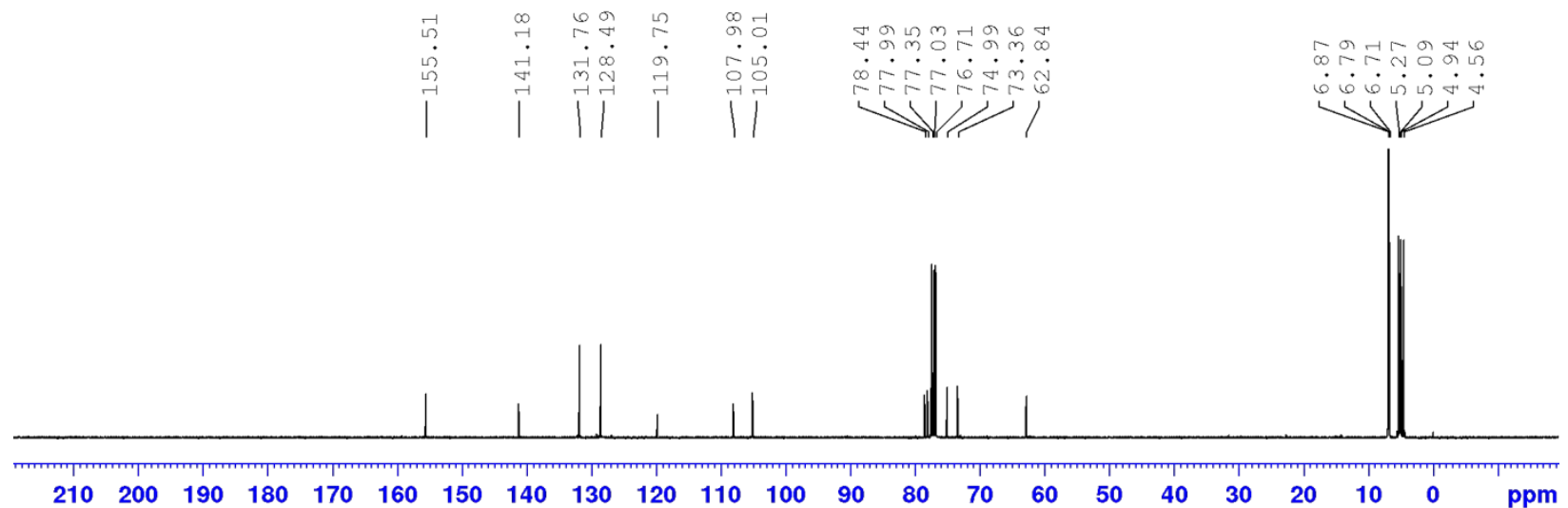


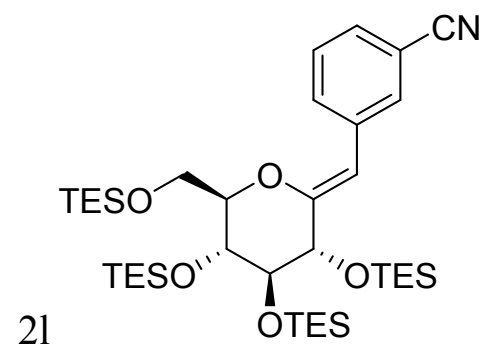

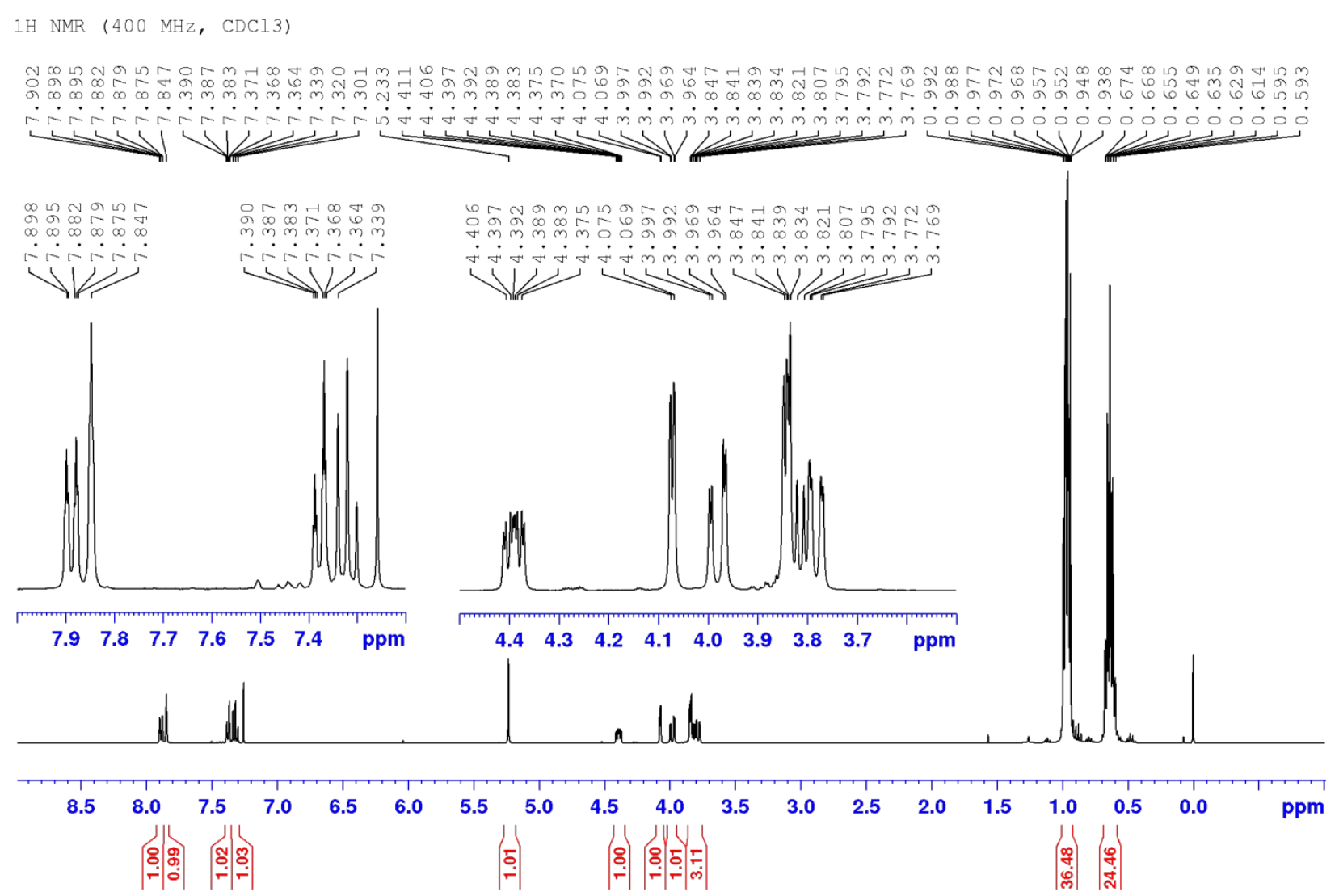

$13 \mathrm{C}\{1 \mathrm{H}\} \mathrm{NMR}(100 \mathrm{MHz}, \mathrm{CDCl} 3)$

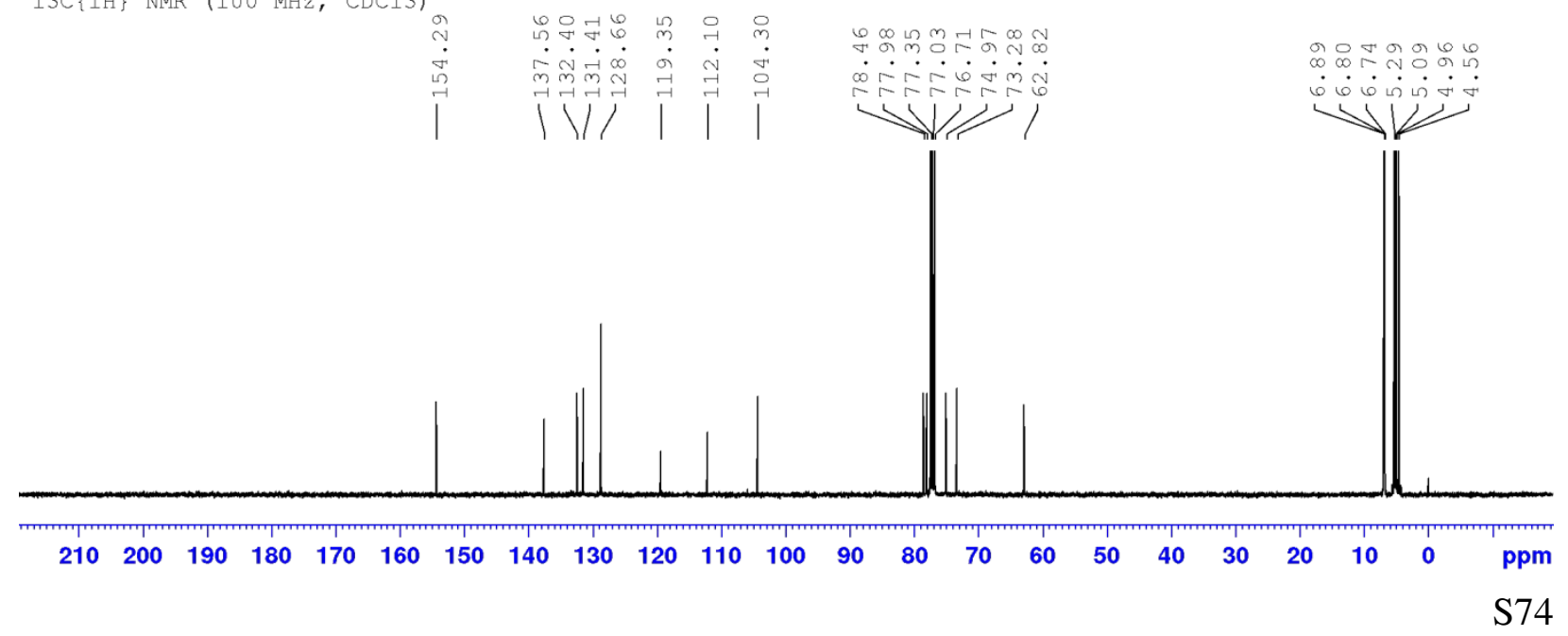




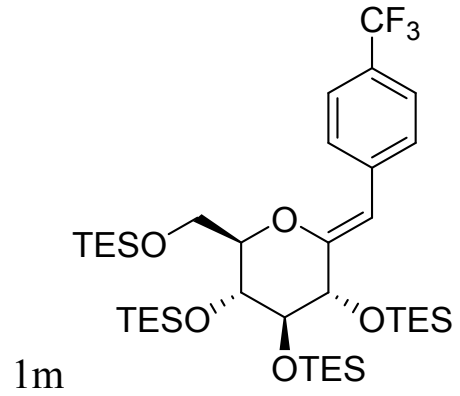

1H NMR (400 MHz, CDCl3)
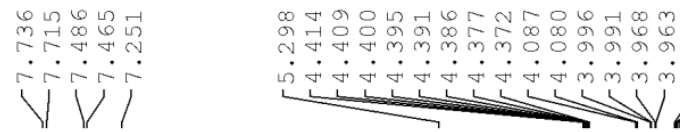

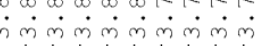

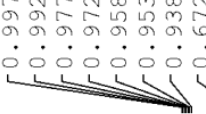

00000000
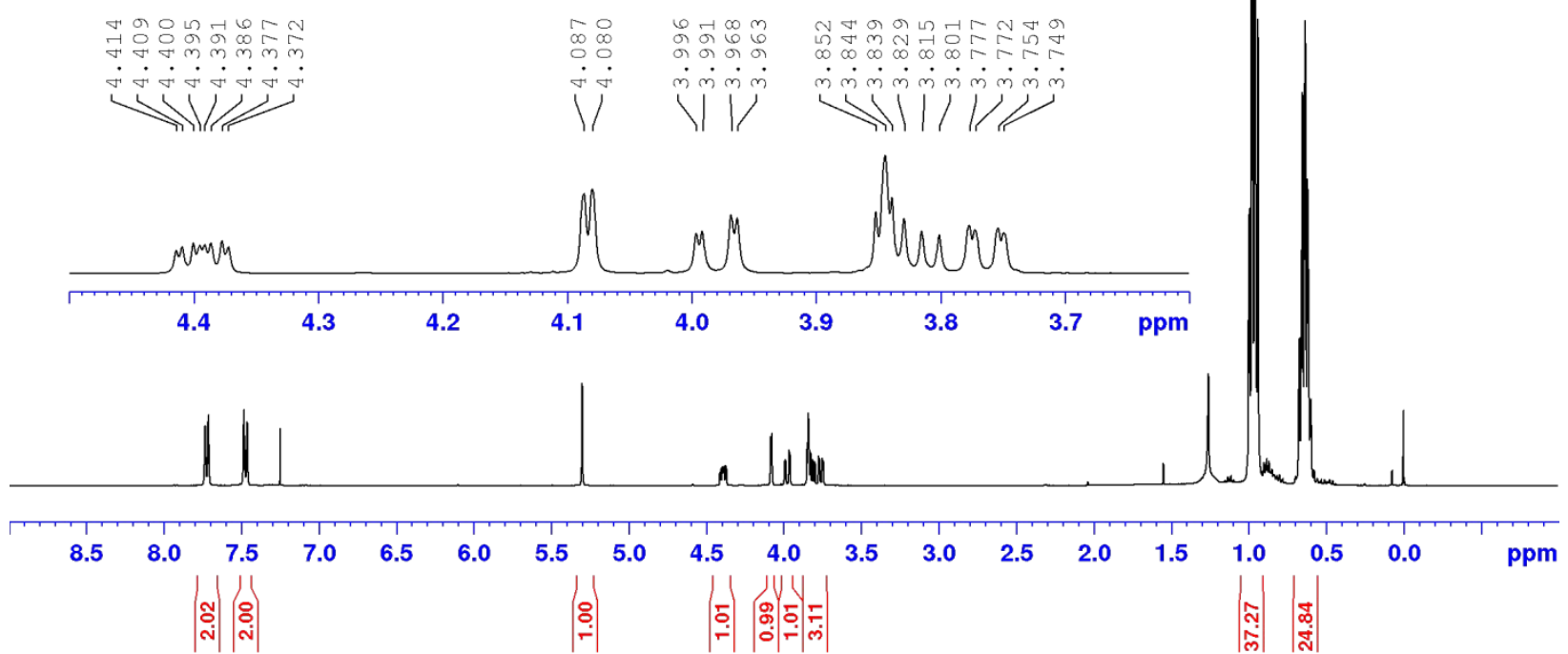

$13 \mathrm{C}\{1 \mathrm{H}\} \mathrm{NMR}(100 \mathrm{MHz}, \mathrm{CDCl} 3)$

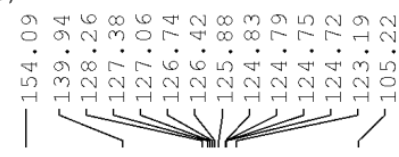

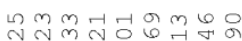

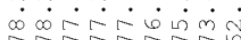

(

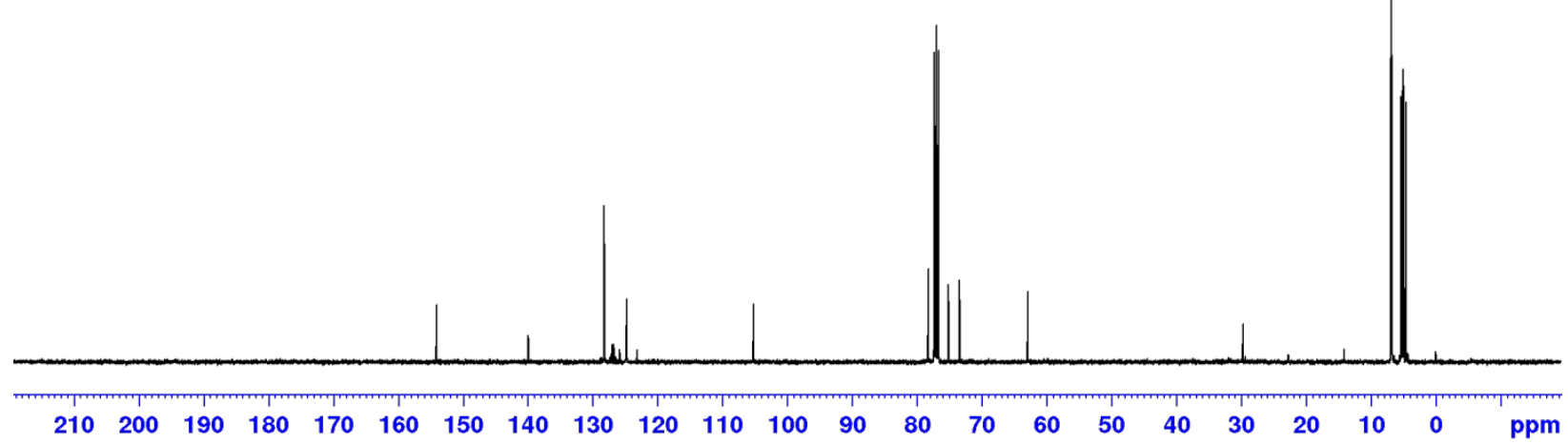




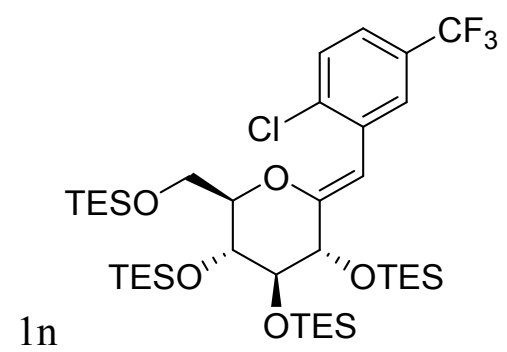

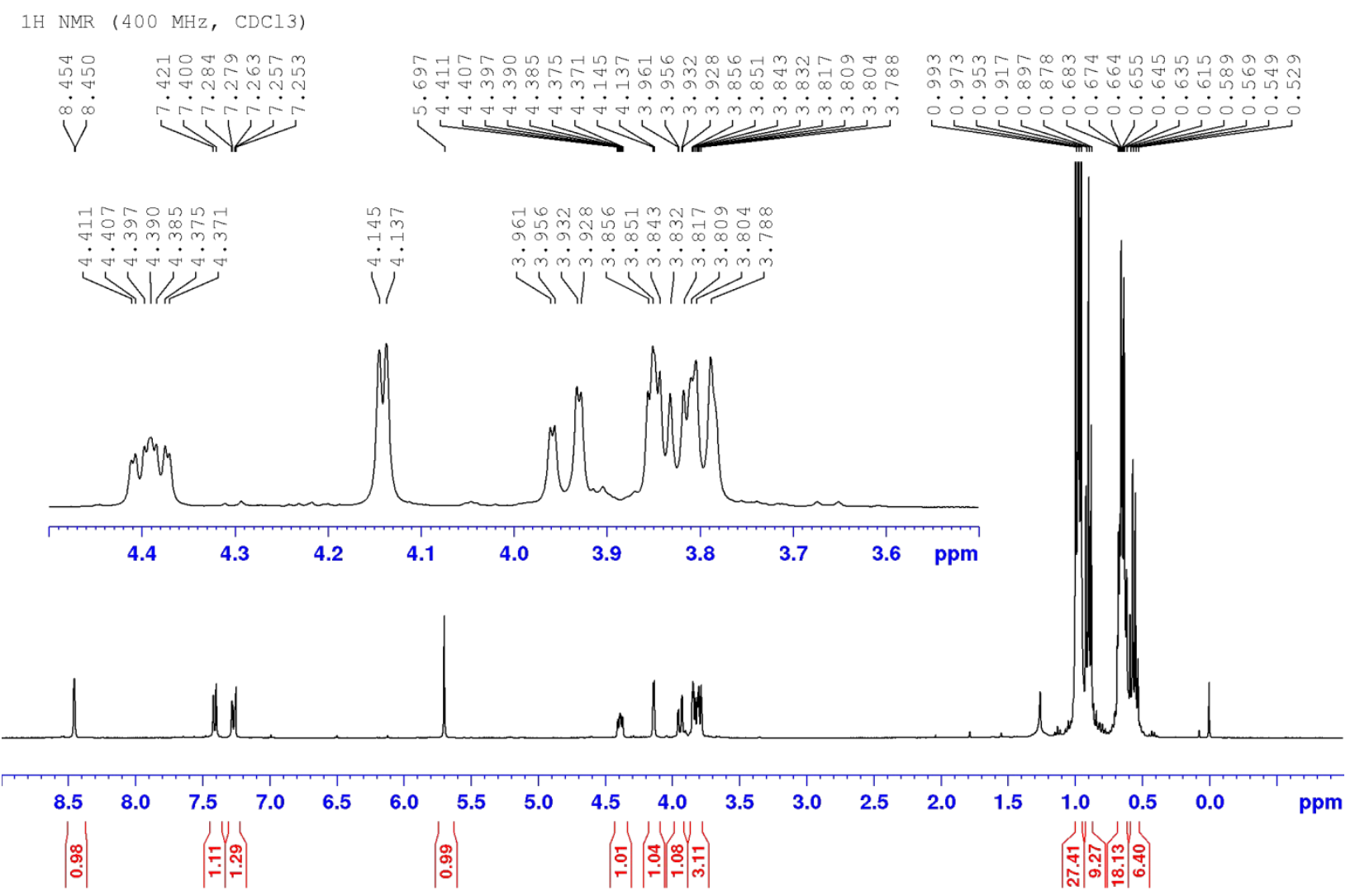




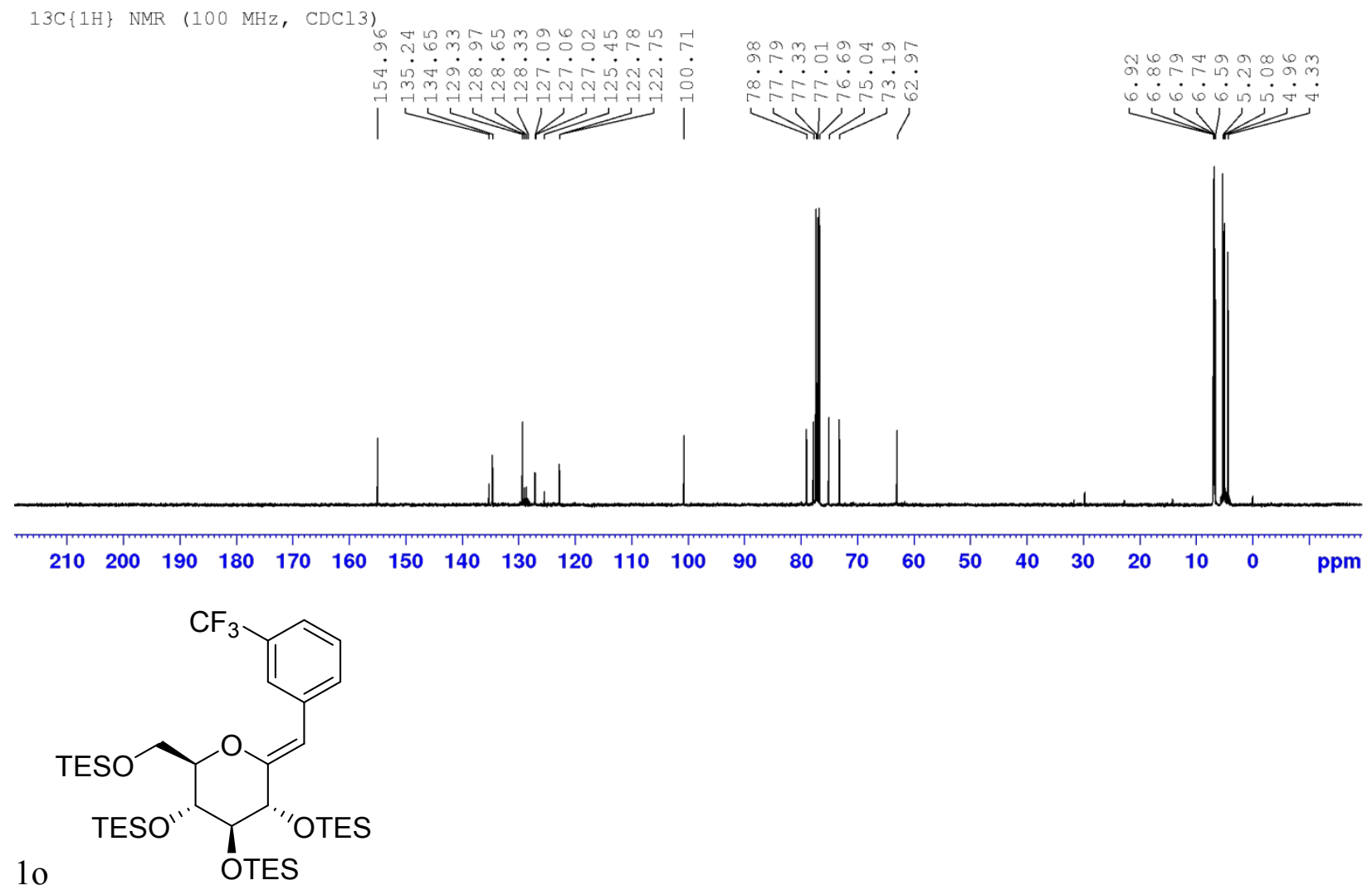


1H NMR (400 MHz, CDCl3)

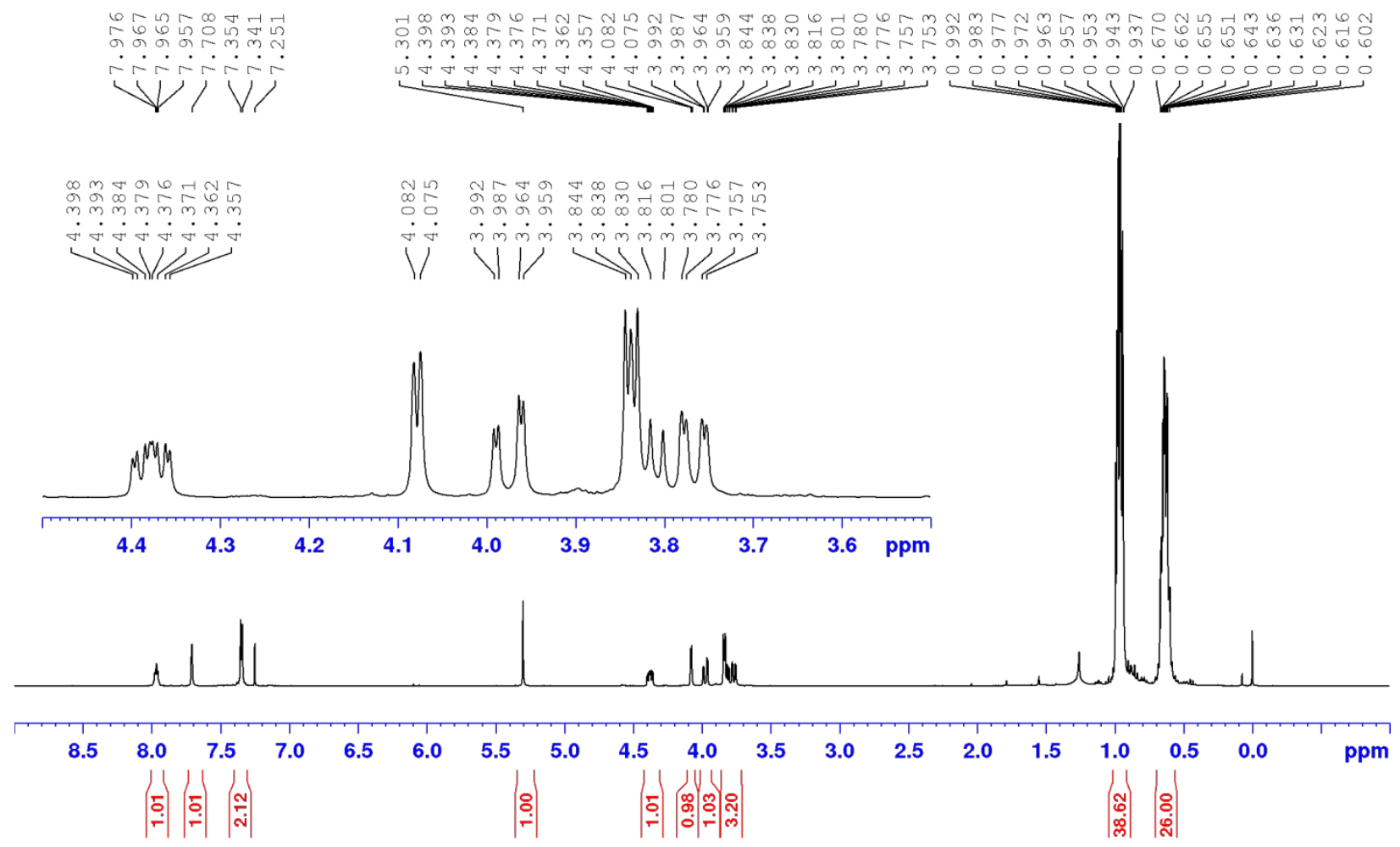

$13 \mathrm{C}\{1 \mathrm{H}\} \mathrm{NMR}(100 \mathrm{MHz}, \mathrm{CDCl} 3)$

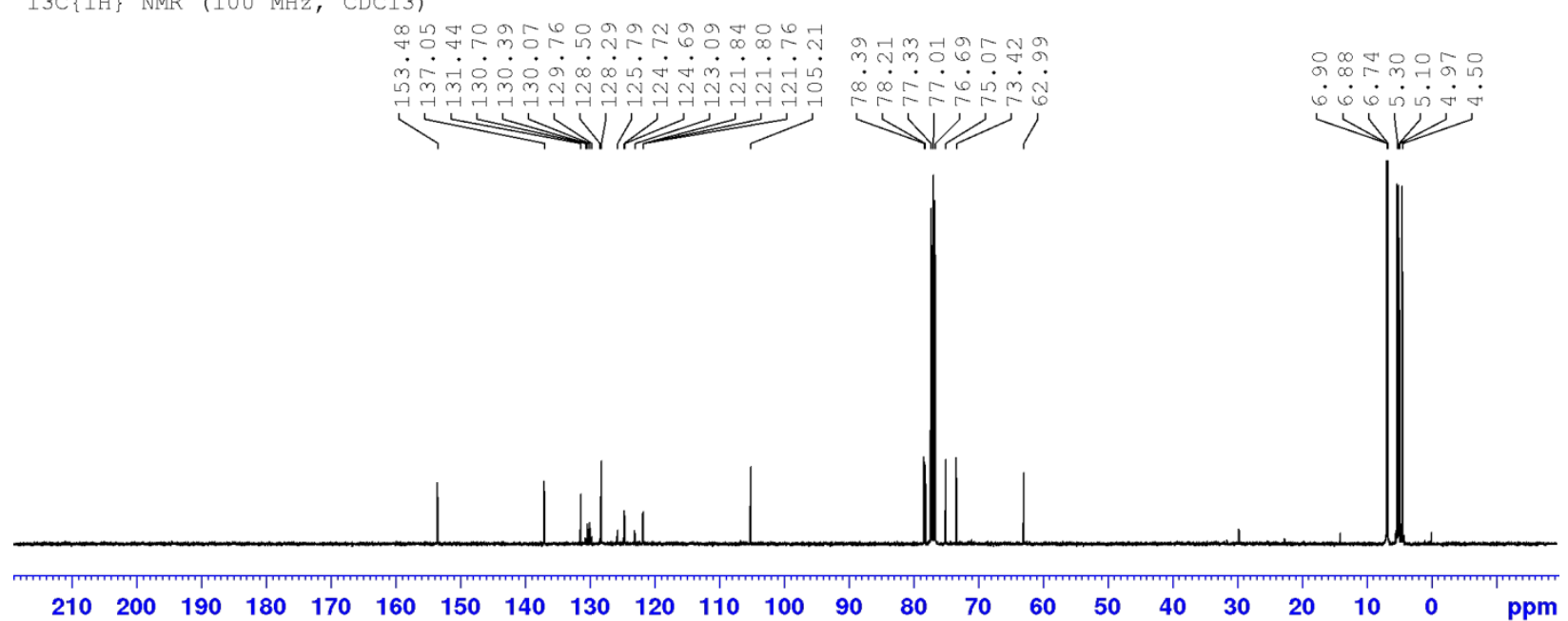




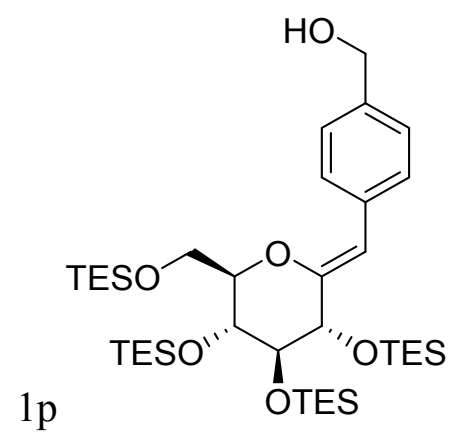

$1 \mathrm{H}$ NMR (400 MHz, CDCl3)
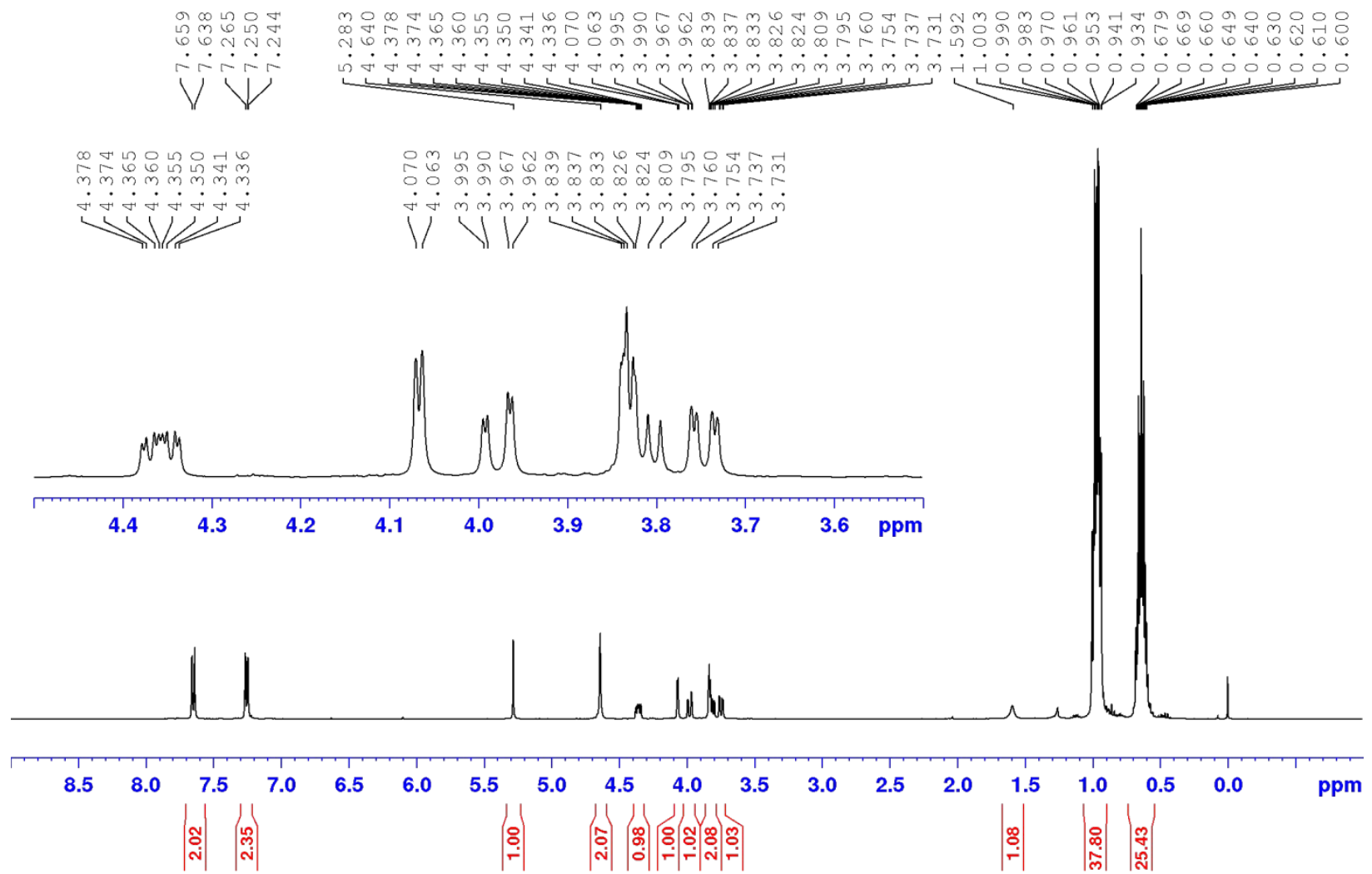


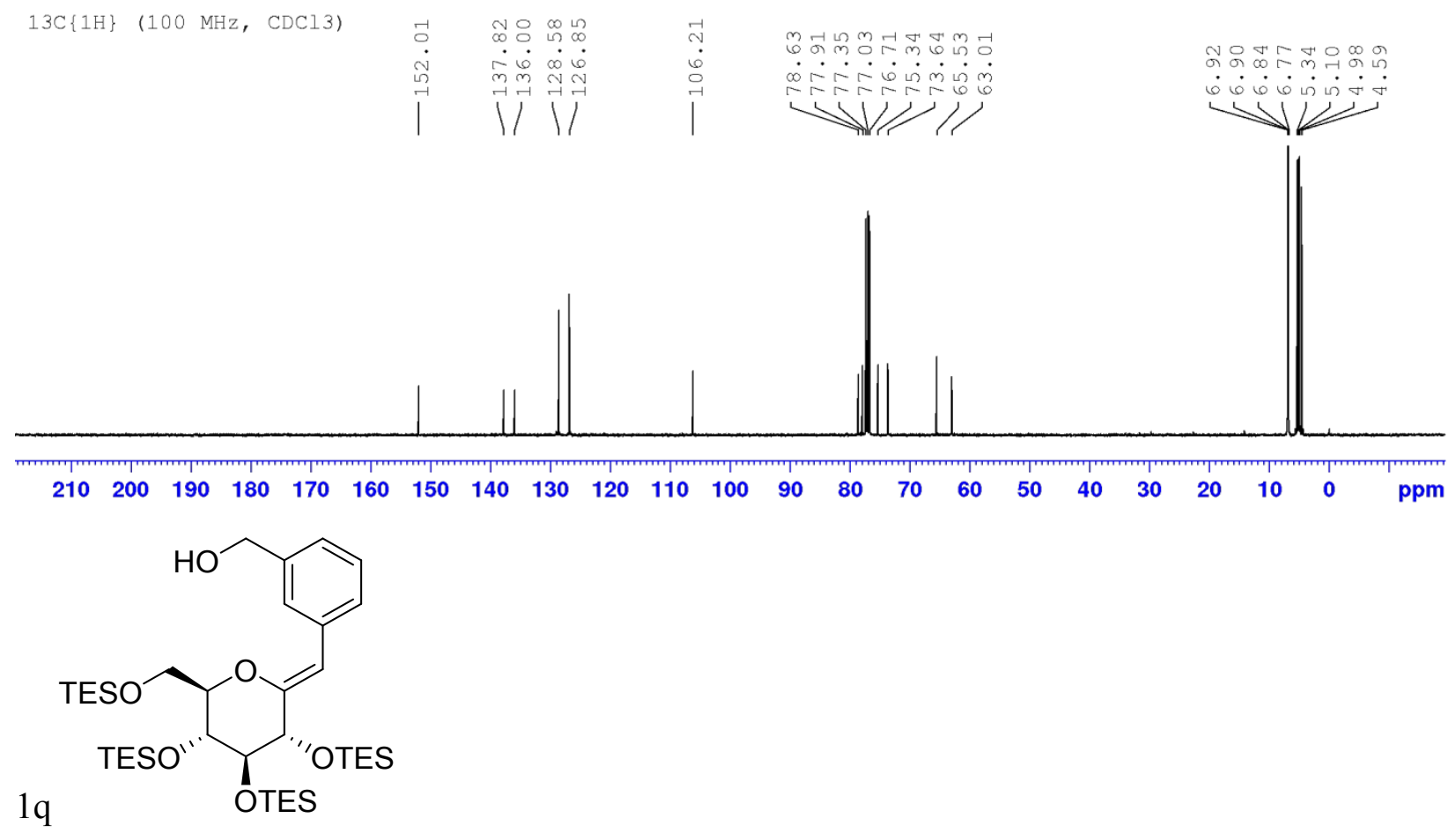

1H NMR (400 MHz, CDCl3)
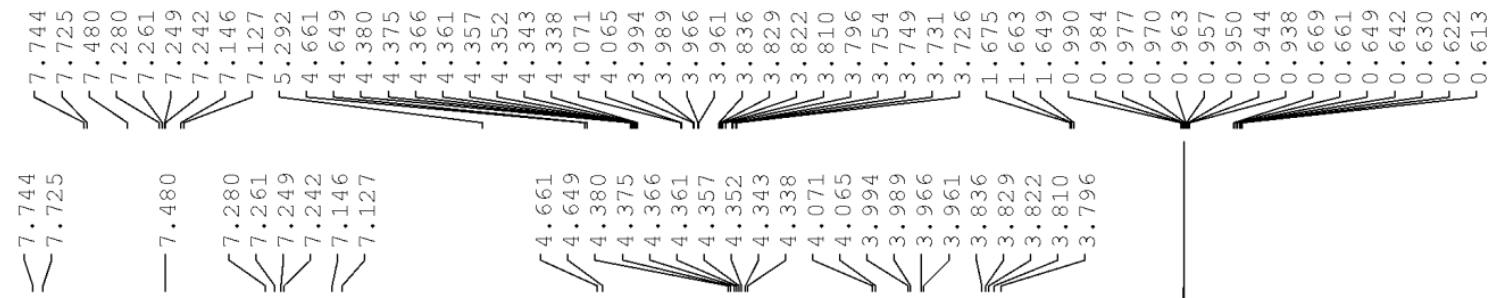

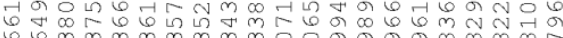

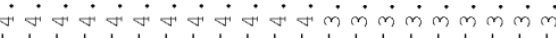

y u V W
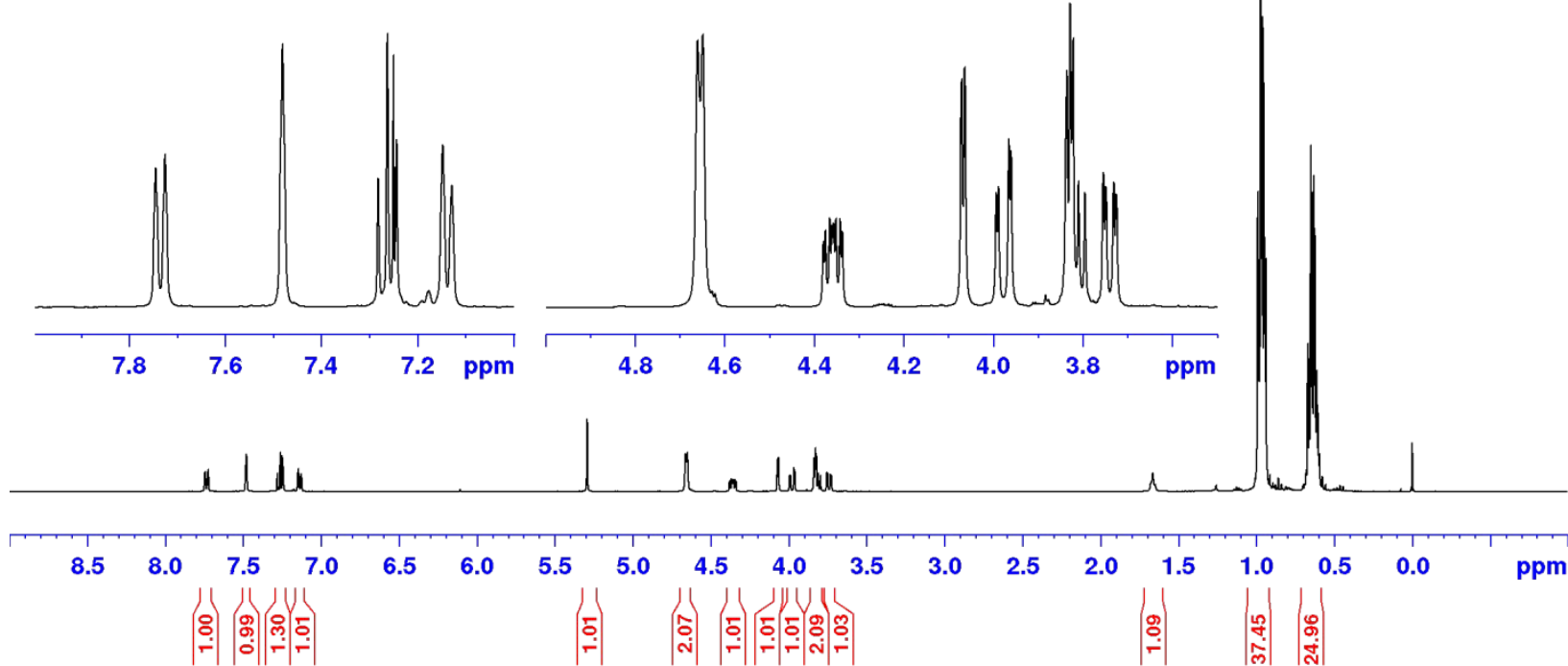


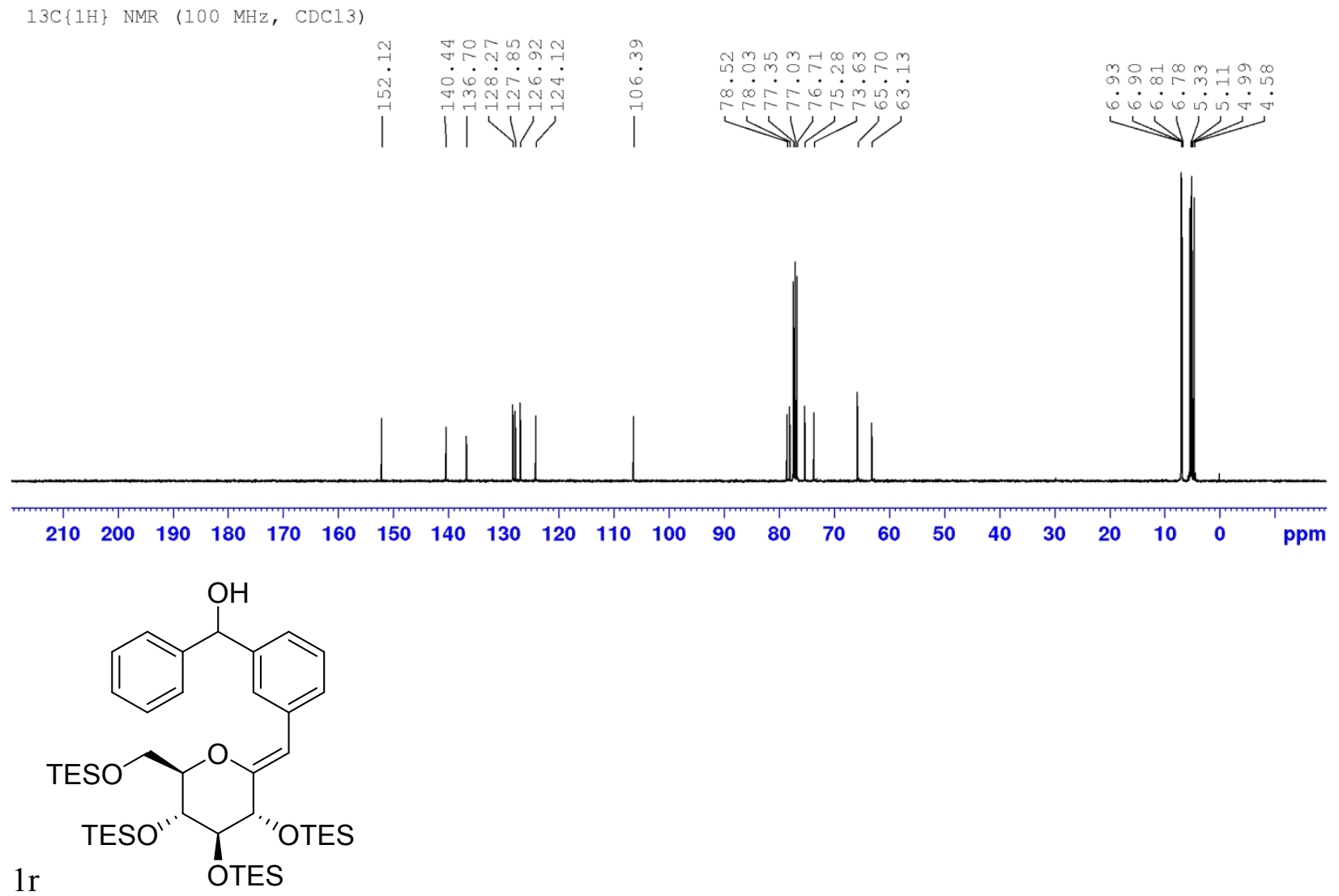


$1 \mathrm{H}$ NMR $(400 \mathrm{MHz}, \mathrm{CDCl} 3)$

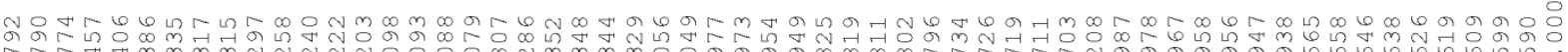

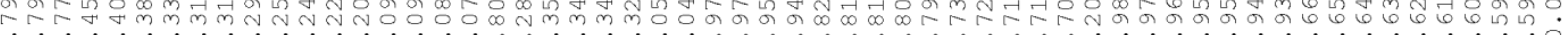

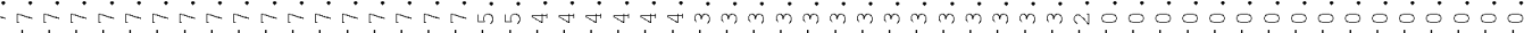
(1)

บ vंगें।

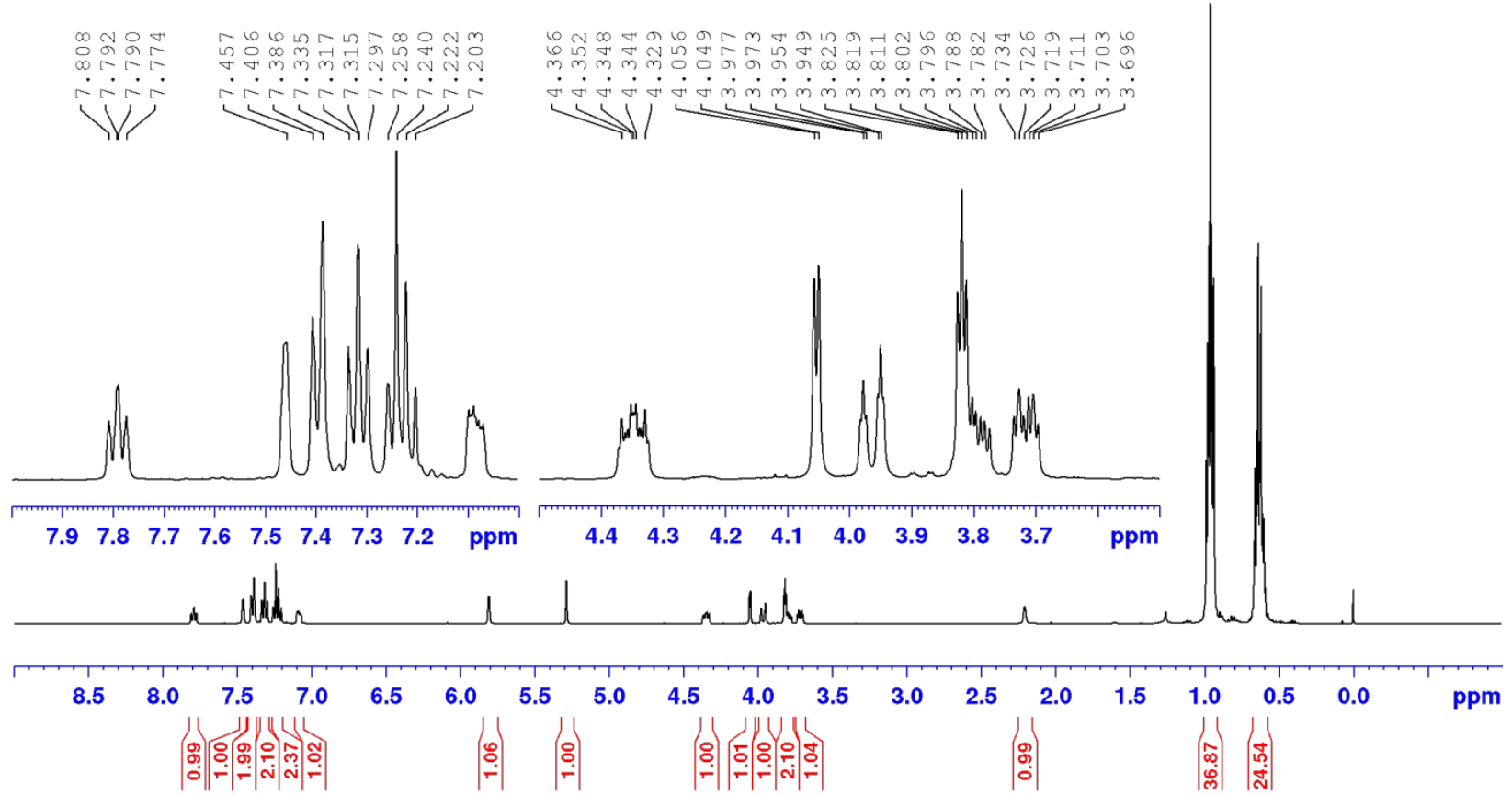

$13 \mathrm{C}\{1 \mathrm{H}\} \operatorname{NMR}(100 \mathrm{MHz}, \mathrm{CDCl} 3)$
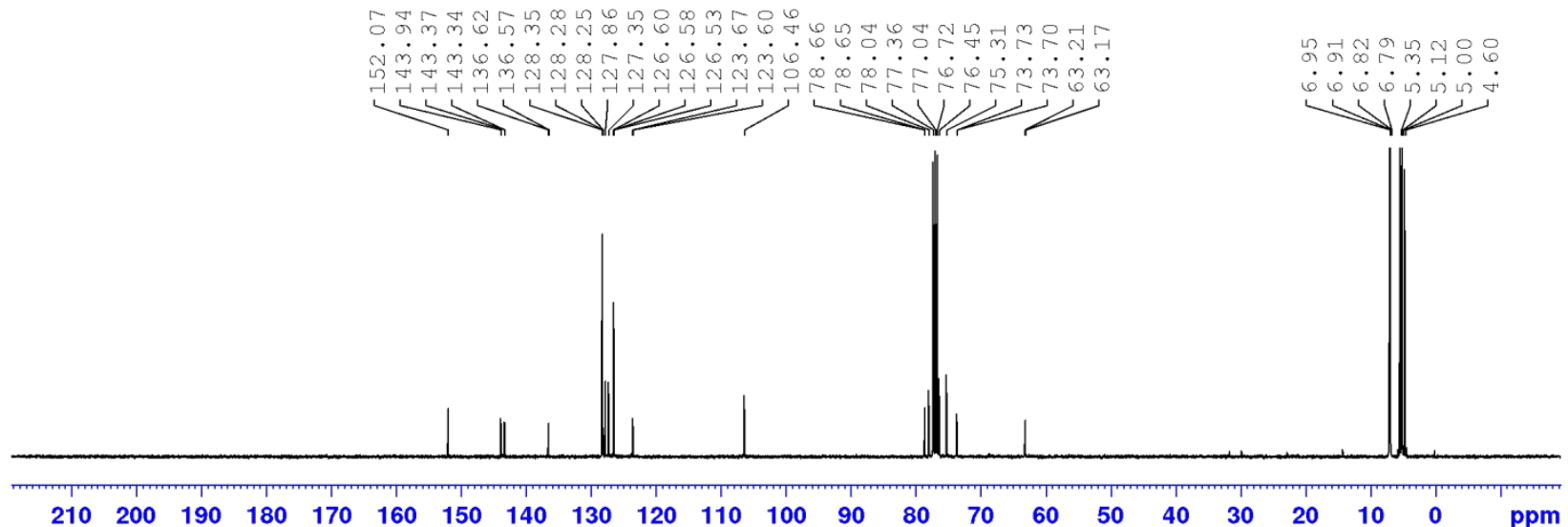

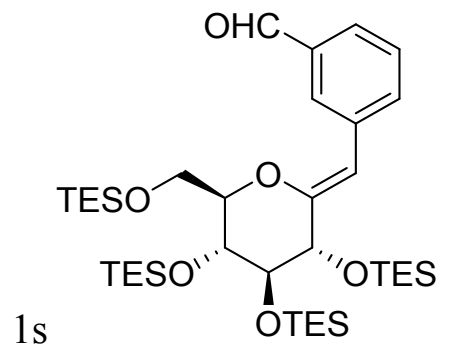


$1 \mathrm{H}$ NMR (400 MHz, CDCl3)
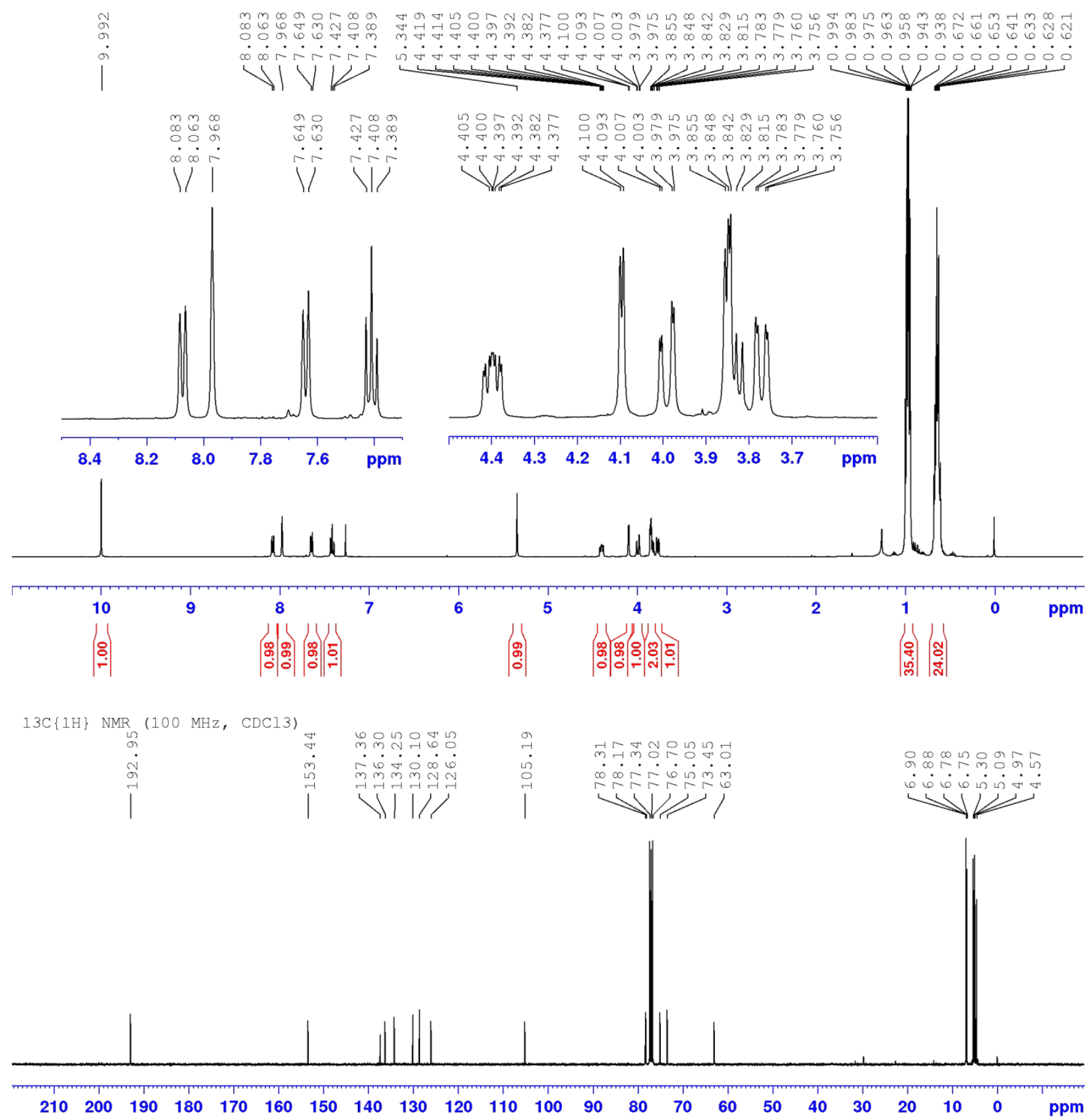


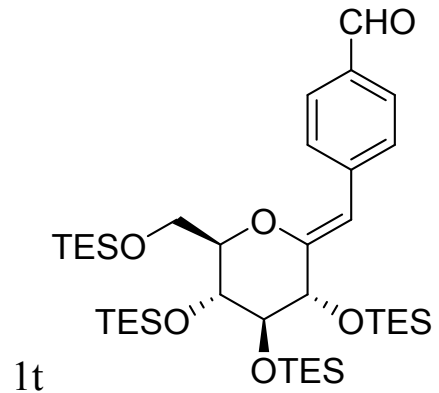

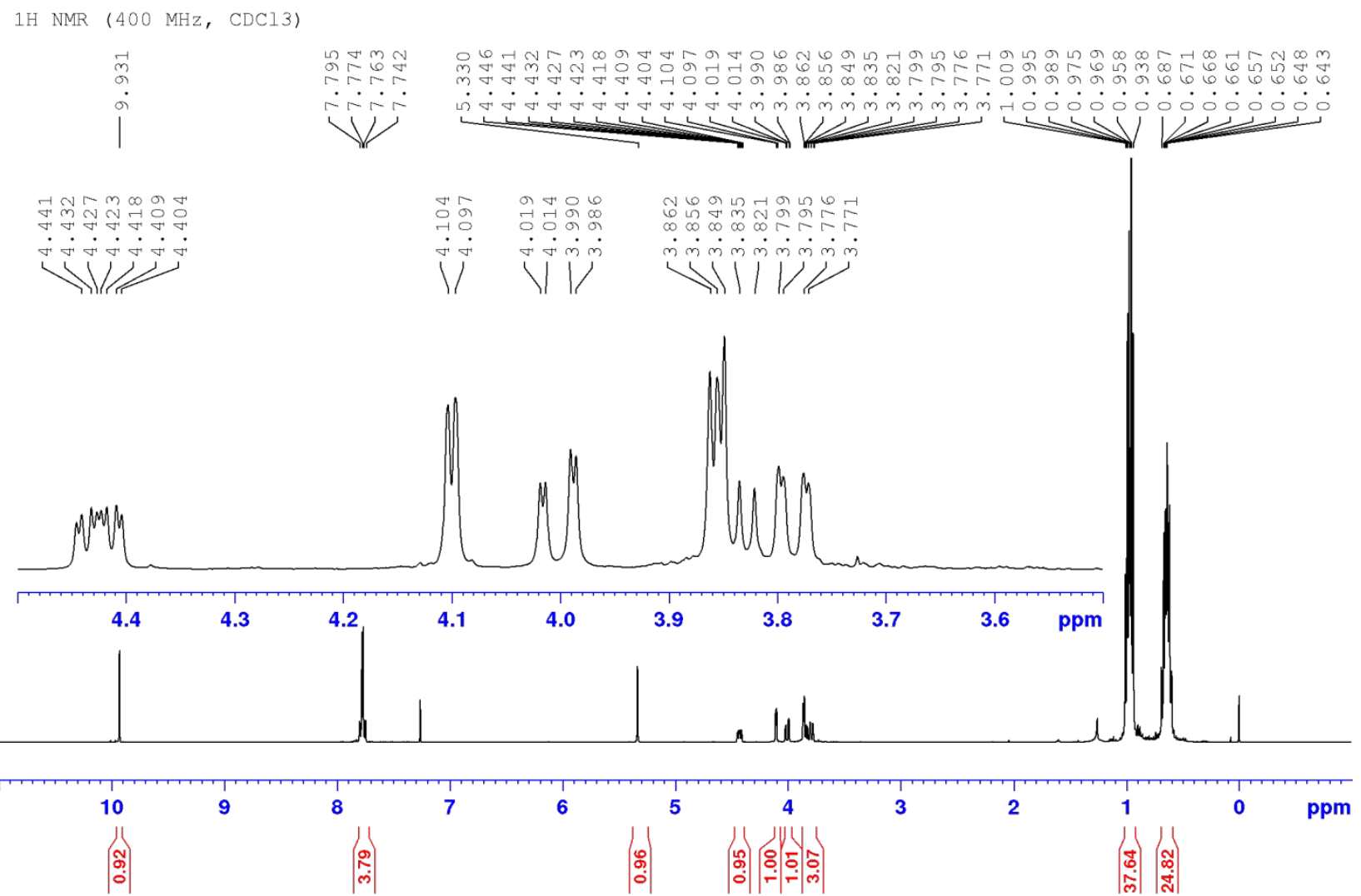

$13 \mathrm{C}\{1 \mathrm{H}\} \mathrm{NMR}(100 \mathrm{MHz}, \mathrm{CDCl} 3)$

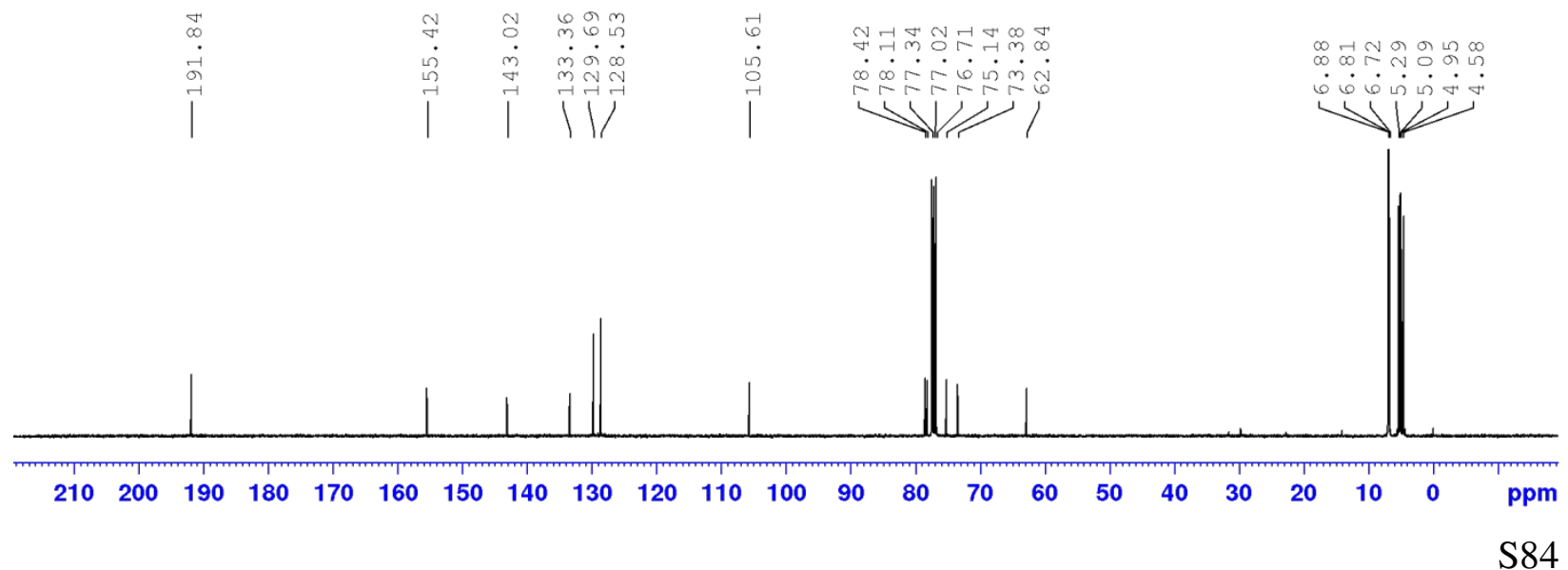




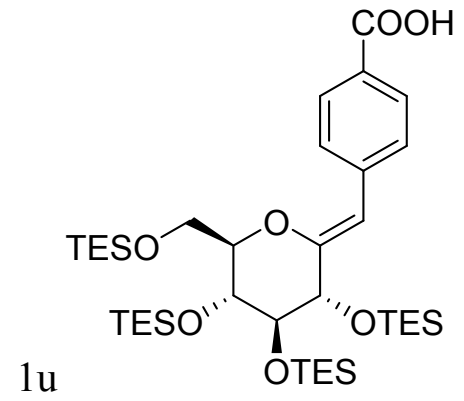

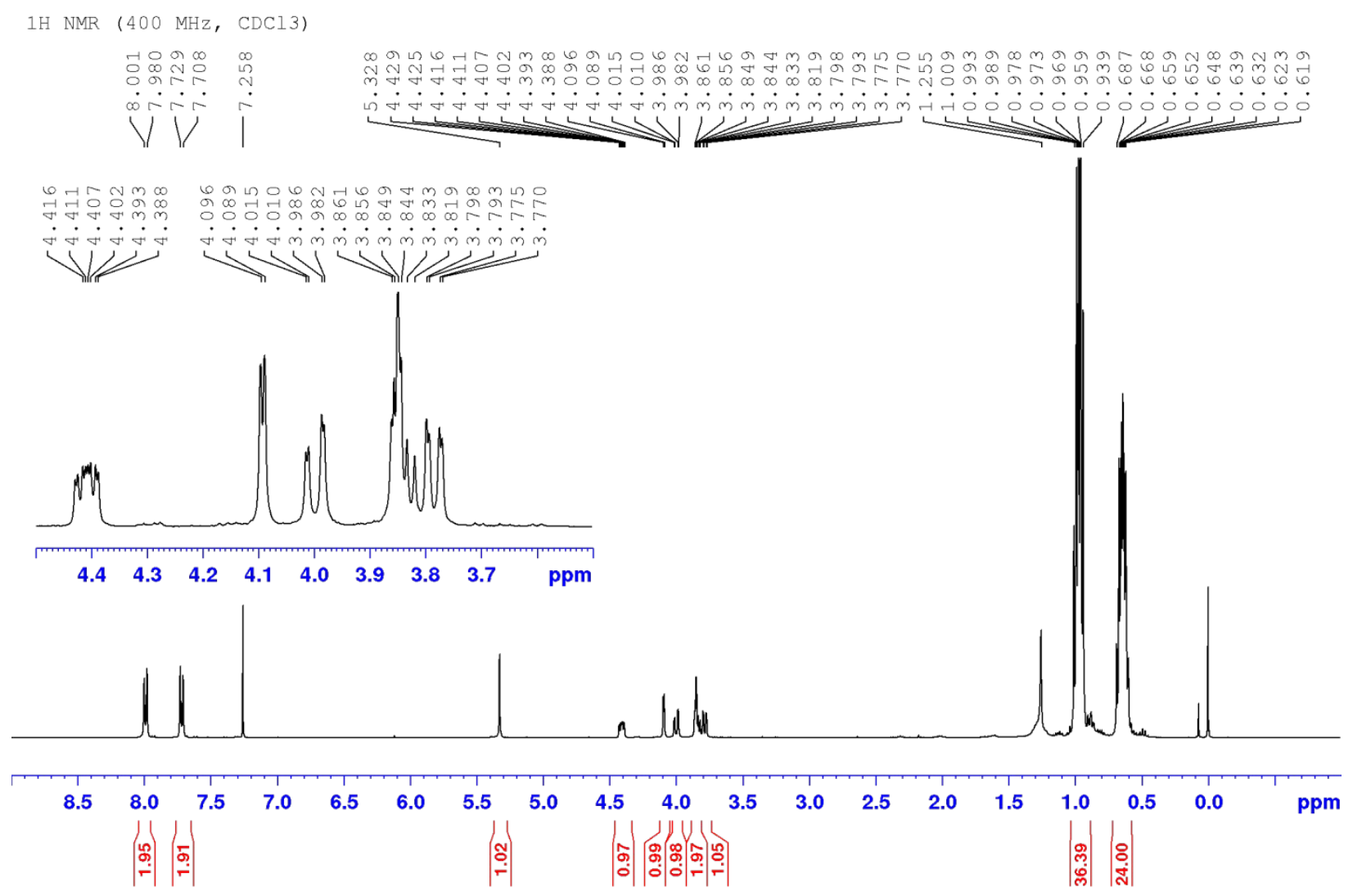



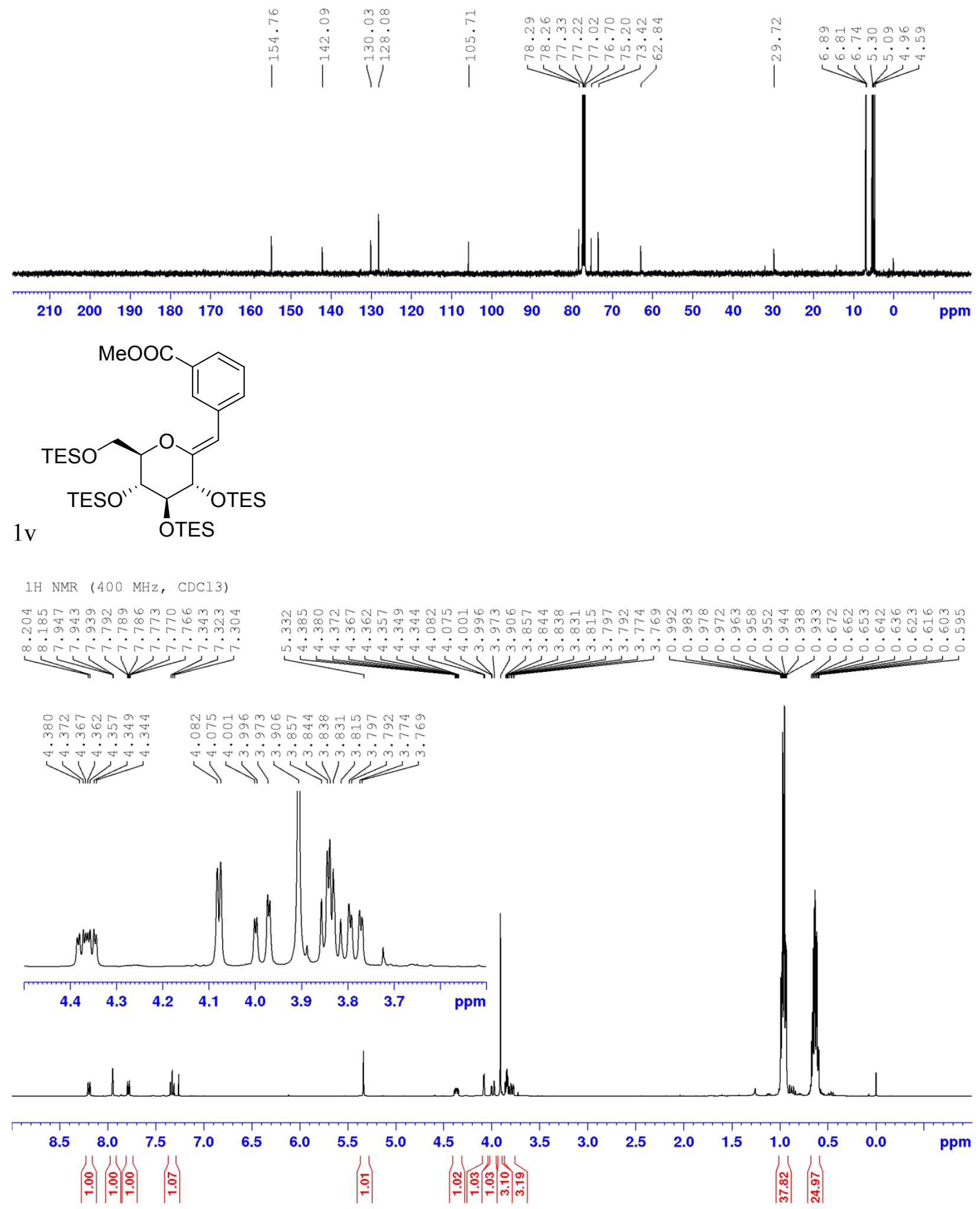

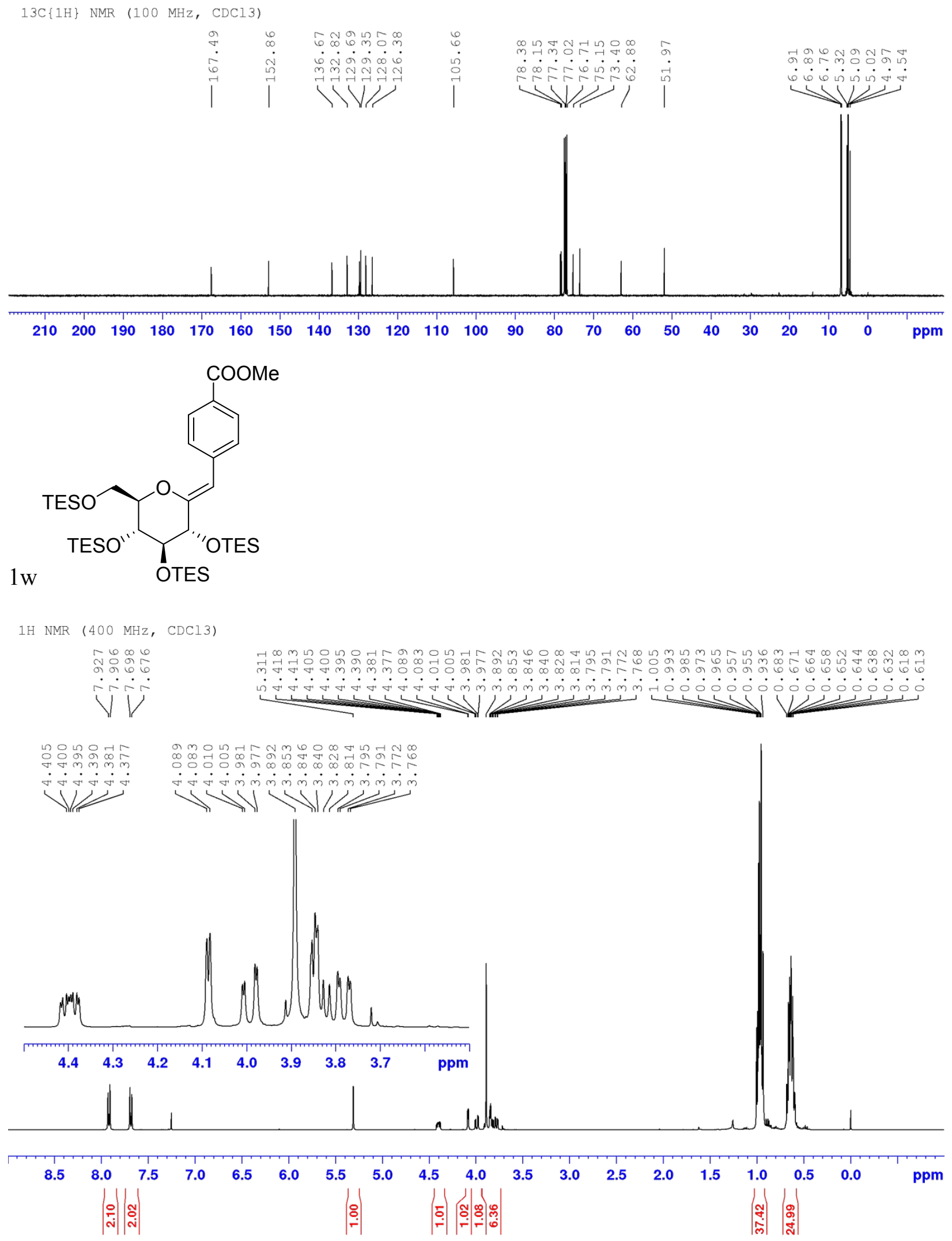
$13 \mathrm{C}\{1 \mathrm{H}\} \mathrm{NMR}(100 \mathrm{MHz}, \mathrm{CDCl} 3)$
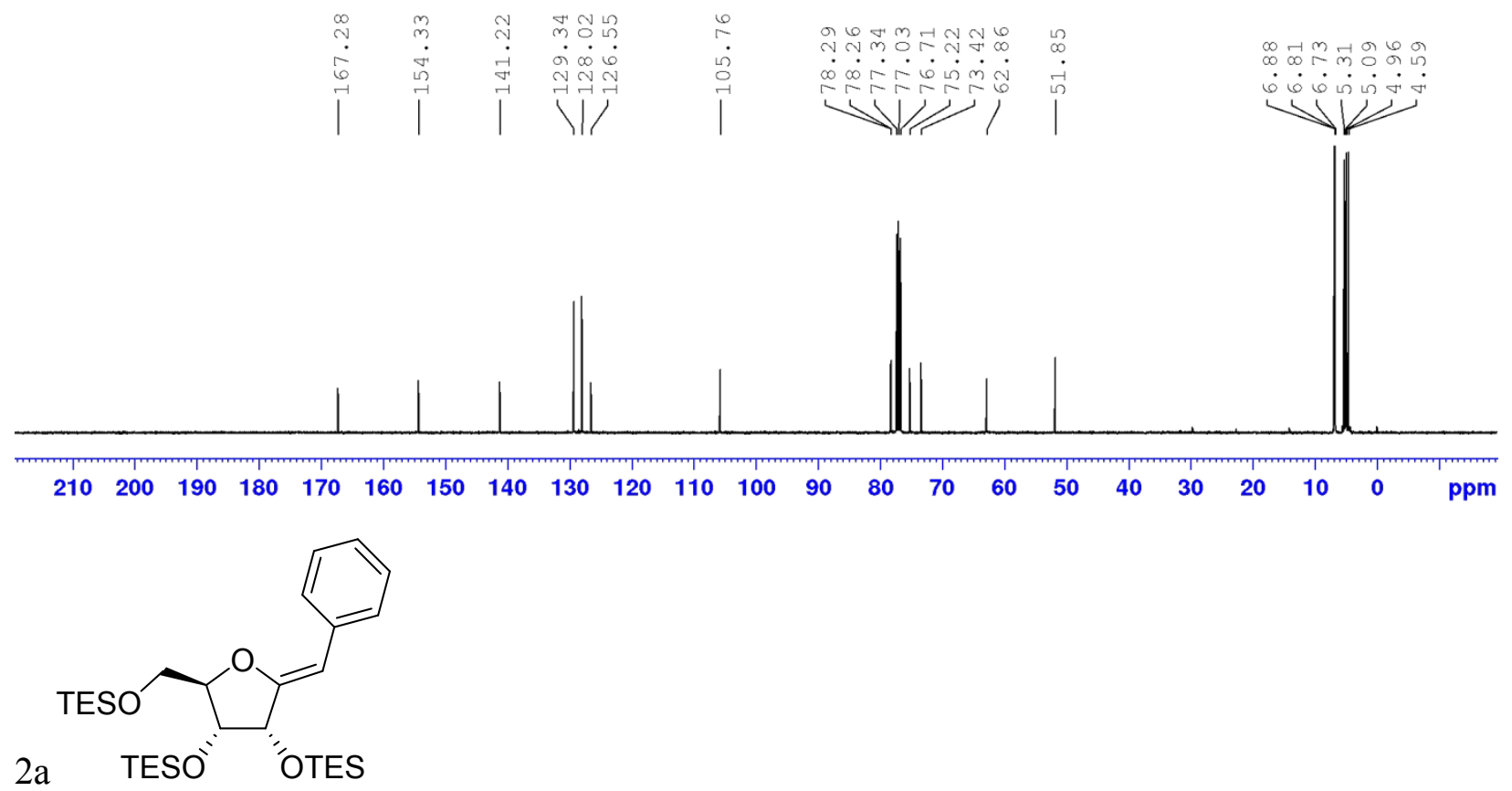

$1 \mathrm{H}$ NMR (400 $\mathrm{MHz}, \mathrm{CDCl} 3)$

6 m 6 m

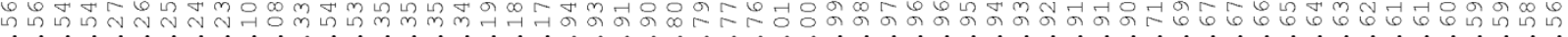

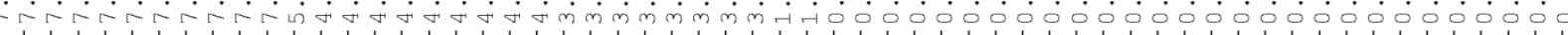

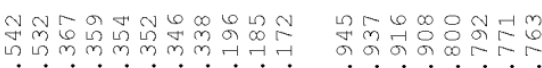

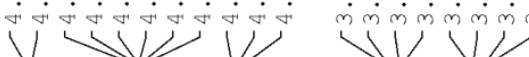
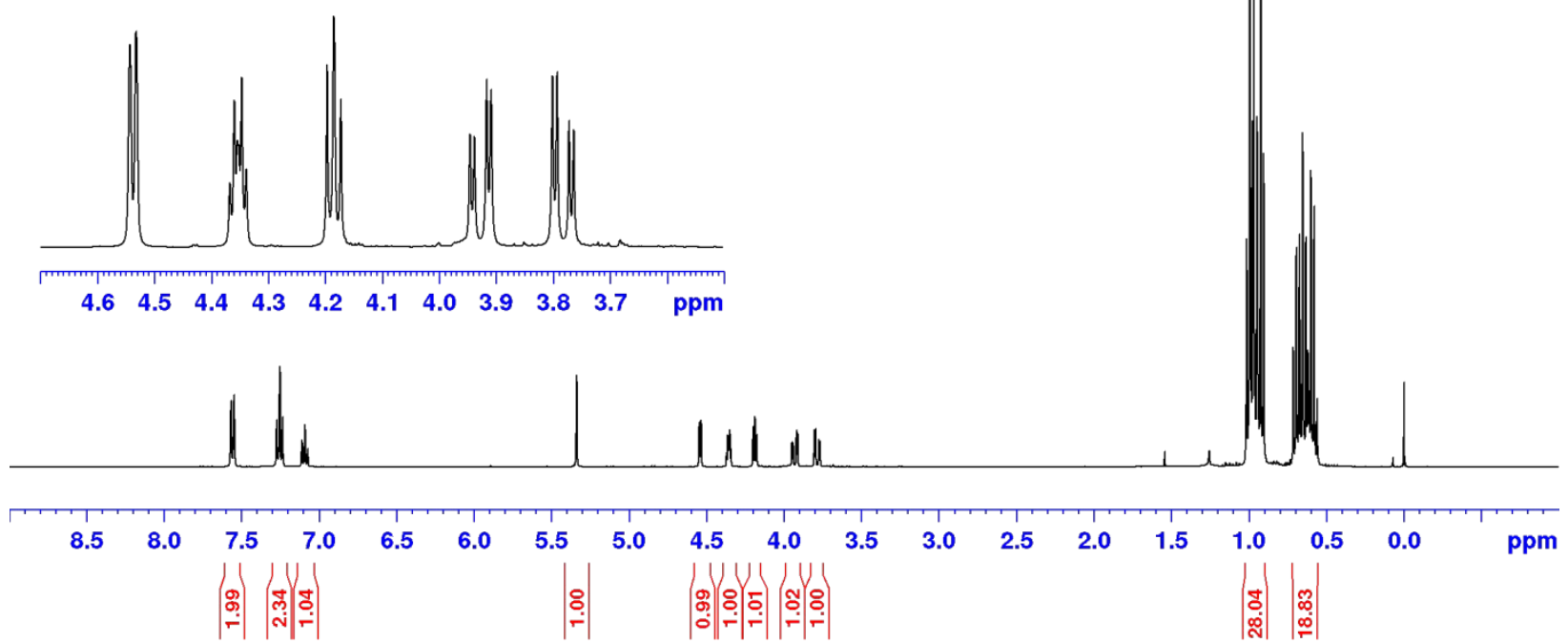
$13 \mathrm{C}\{1 \mathrm{H}\}$ NMR $(100 \mathrm{MHz}, \mathrm{CDCl} 3)$

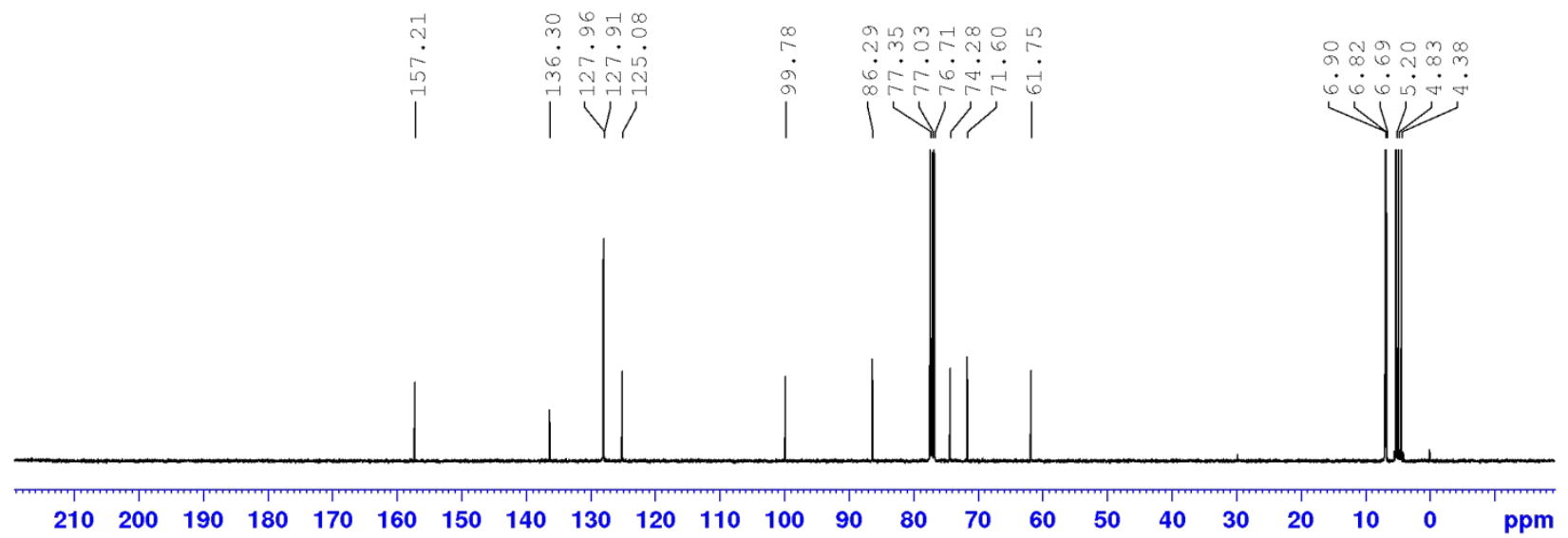<smiles>O=[N+]([O-])c1cccc(/C=C2\O[C@H](COS(F)(F)F)[C@H](OC#[SH])[C@H]2OC#[SH])c1</smiles>

$1 \mathrm{H} \operatorname{NMR}(400 \mathrm{MHz}, \mathrm{CDCl} 3)$

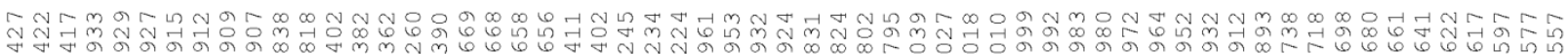

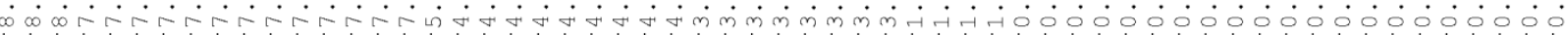

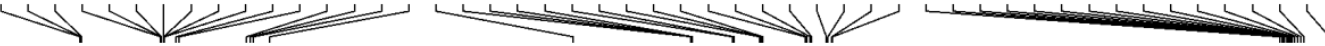
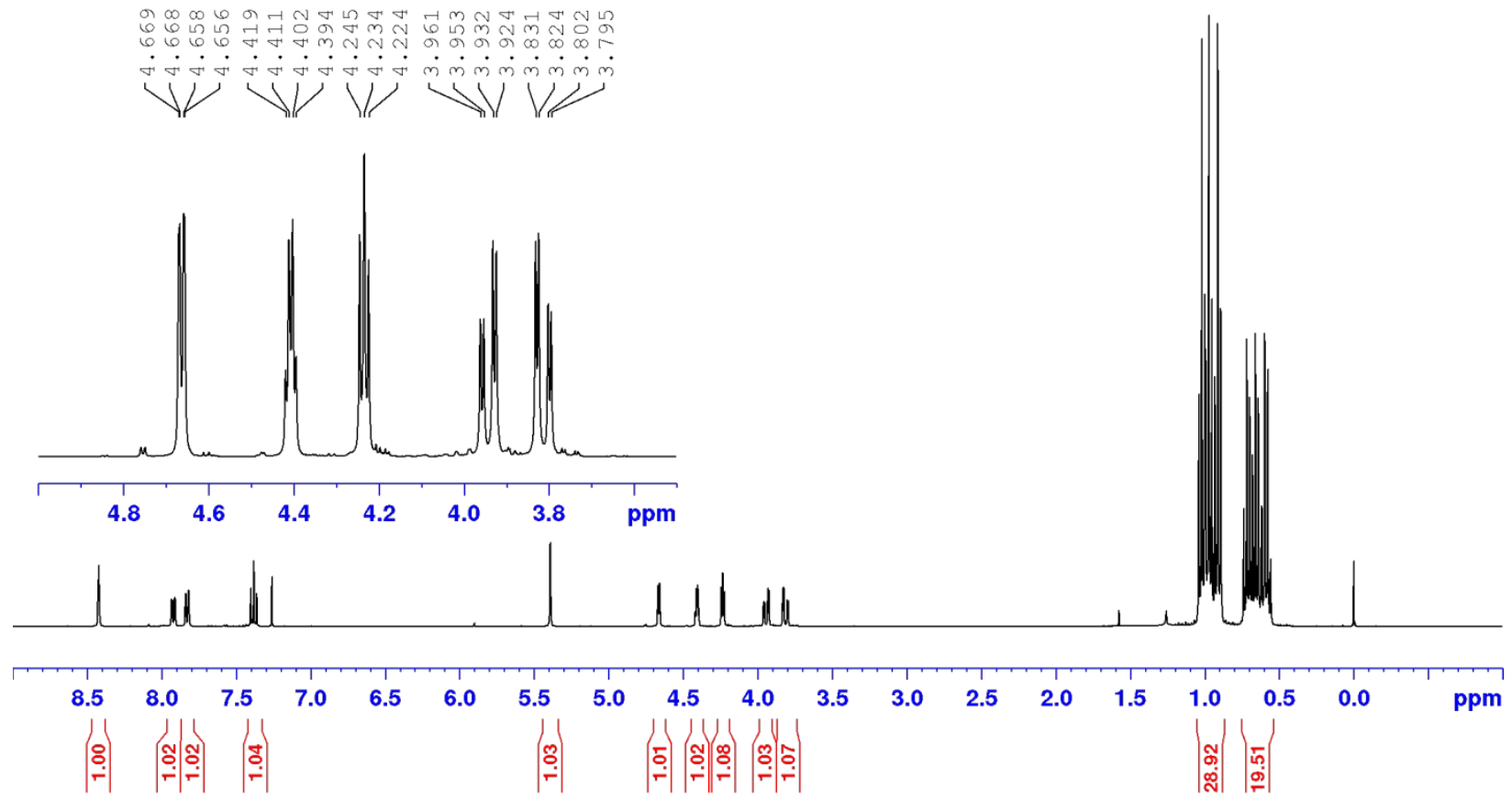


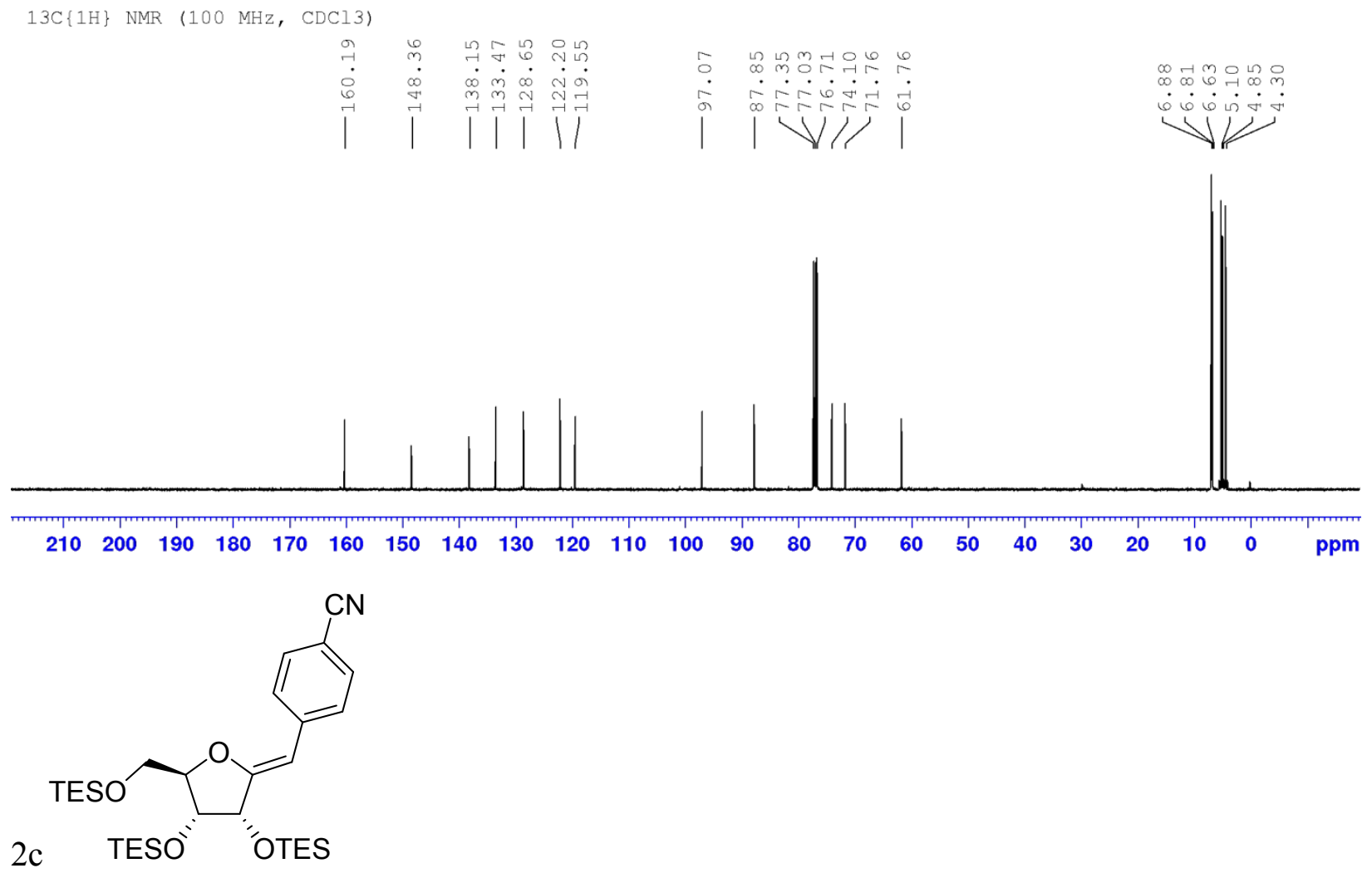


1H NMR (400 MHz, CDC13)
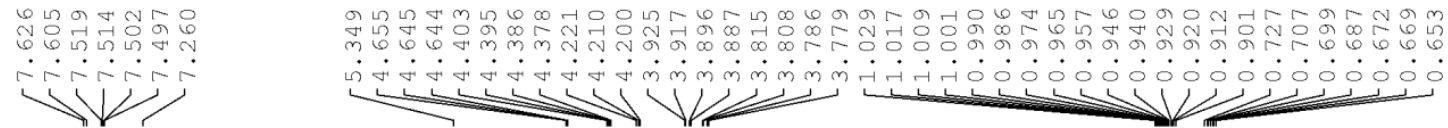

$+1$

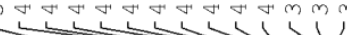

m이에

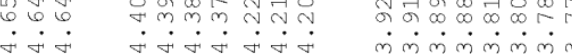

V W W W W W W

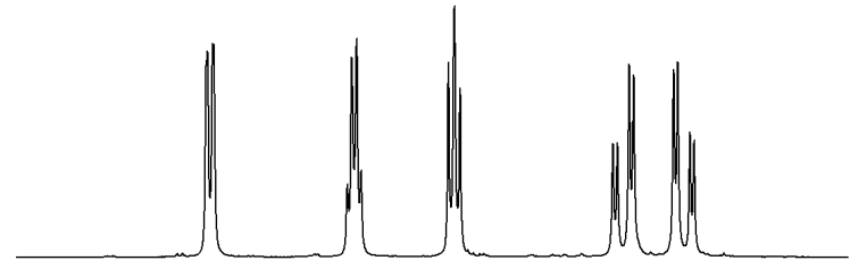

$\begin{array}{llllllllllllll}4.9 & 4.8 & 4.7 & 4.6 & 4.5 & 4.4 & 4.3 & 4.2 & 4.1 & 4.0 & 3.9 & 3.8 & 3.7 & \text { ppm }\end{array}$
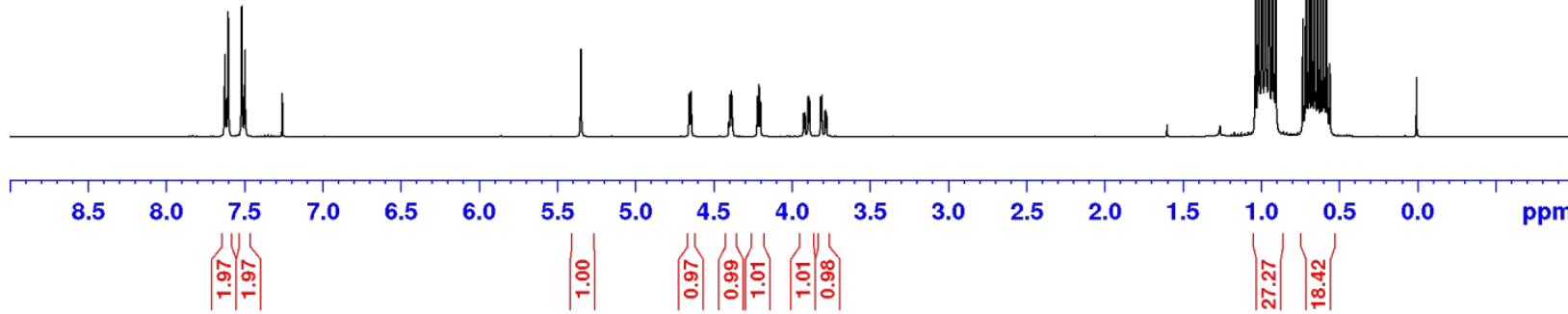

$13 \mathrm{C}\{1 \mathrm{H}\} \mathrm{NMR}(100 \mathrm{MHz}, \mathrm{CDCl} 3)$

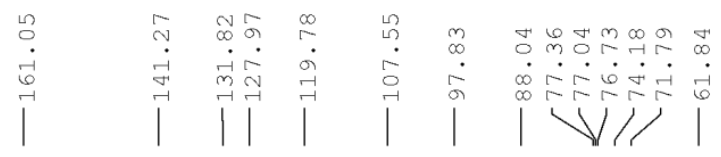

0
$\infty$

$\dot{\omega} \dot{\varphi} \dot{\omega} \dot{\sigma}$

No

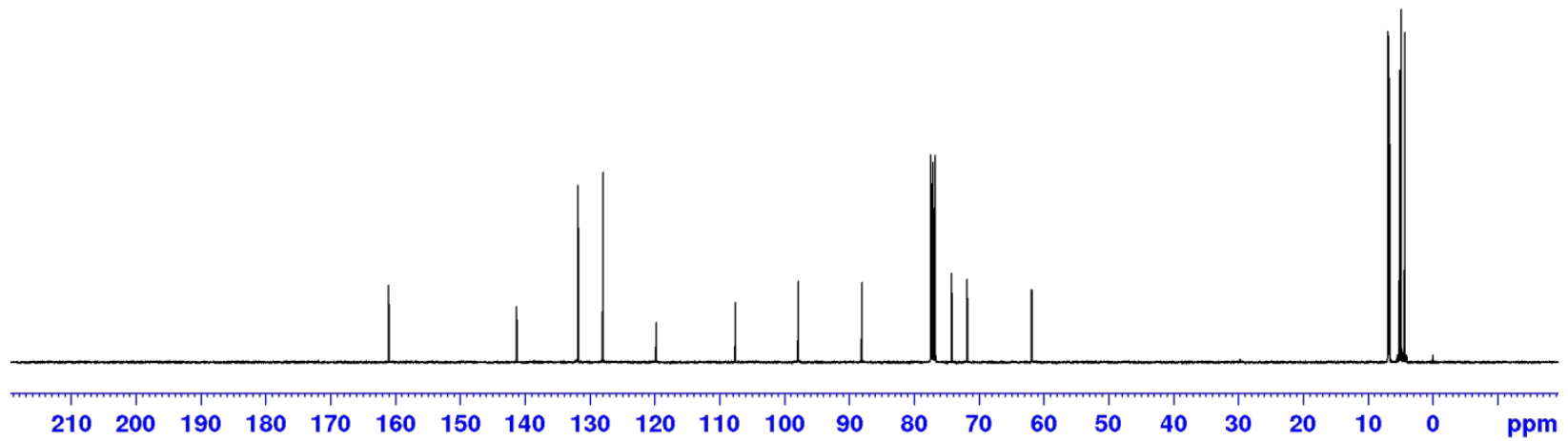



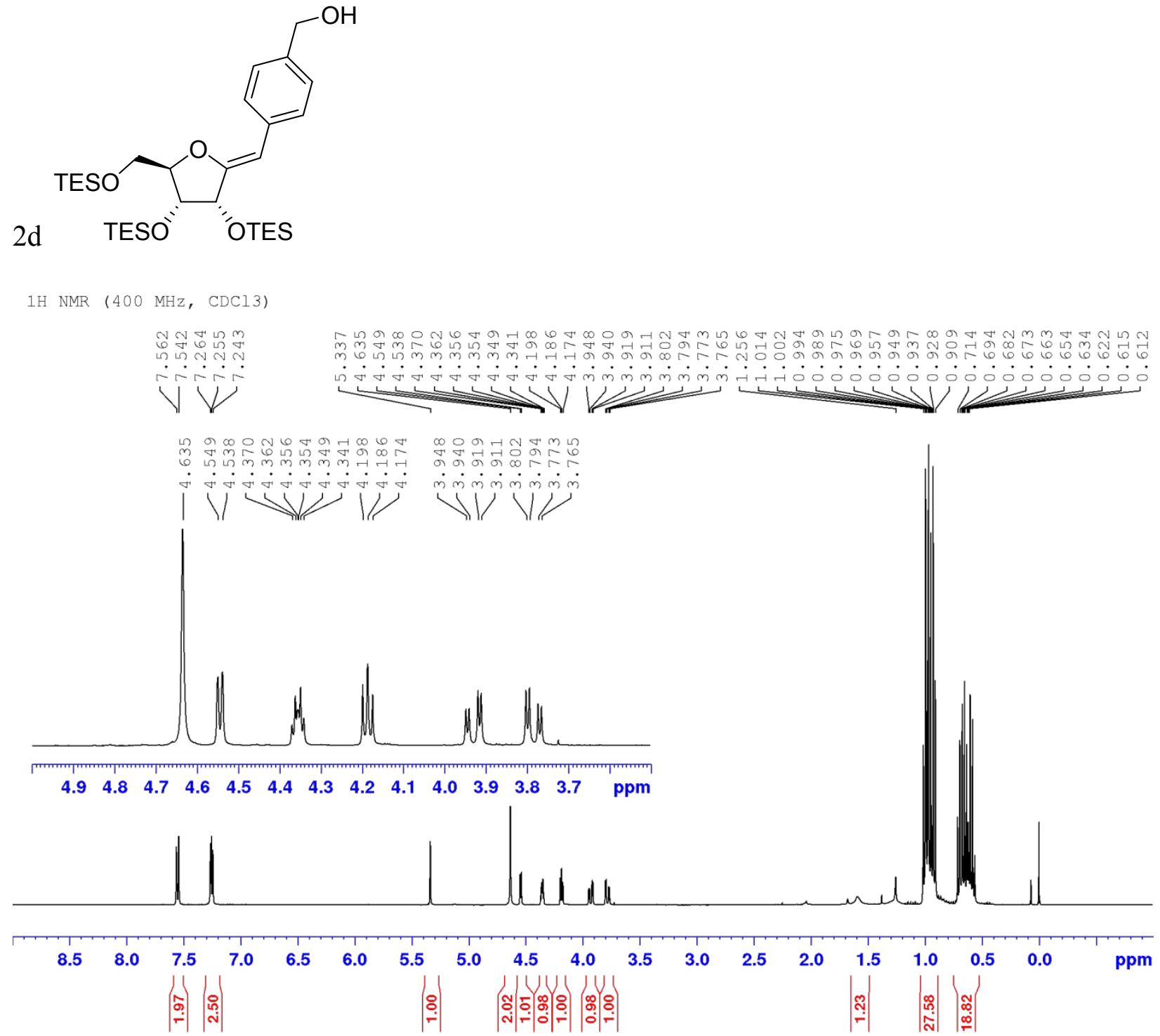


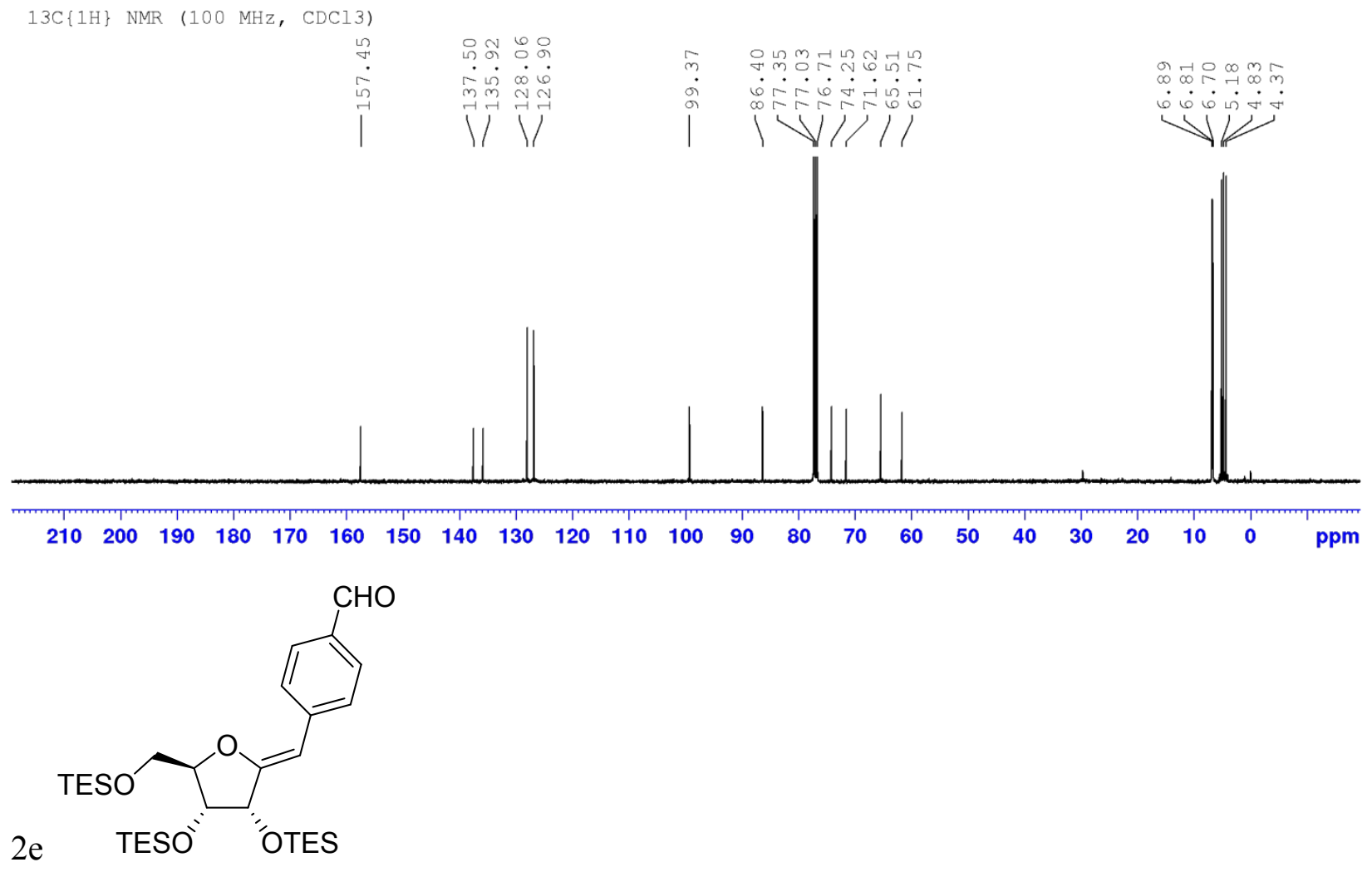


1H NMR (400 MHz, CDCl3)

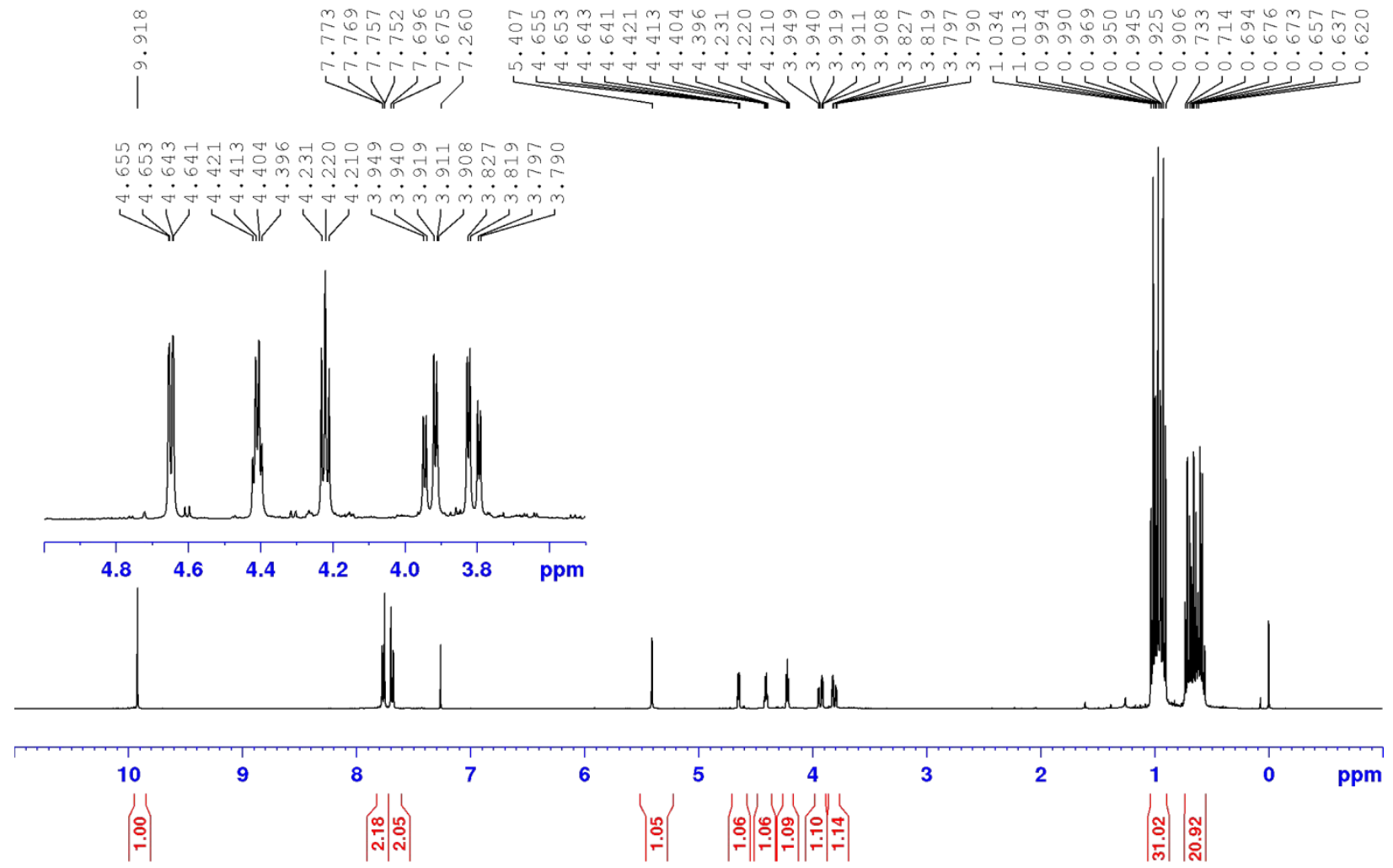

$13 \mathrm{C}\{1 \mathrm{H}\} \mathrm{NMR}(100 \mathrm{MHz}, \mathrm{CDCl} 3)$
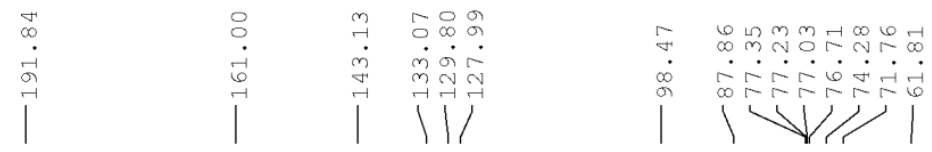

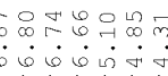
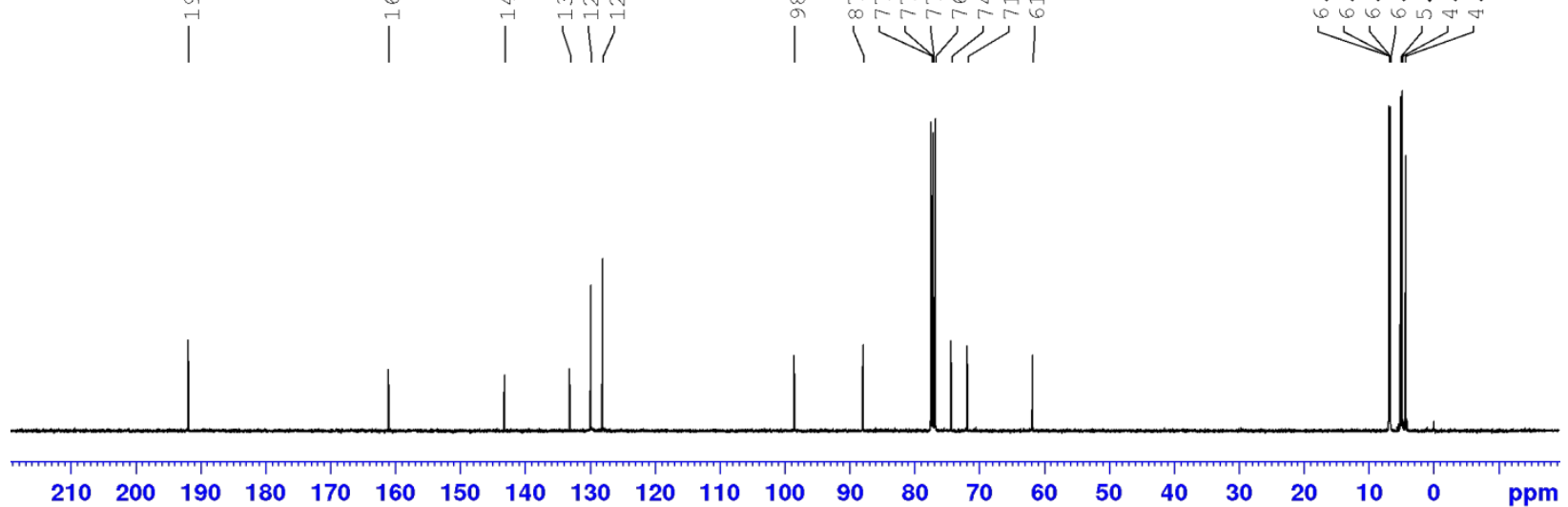

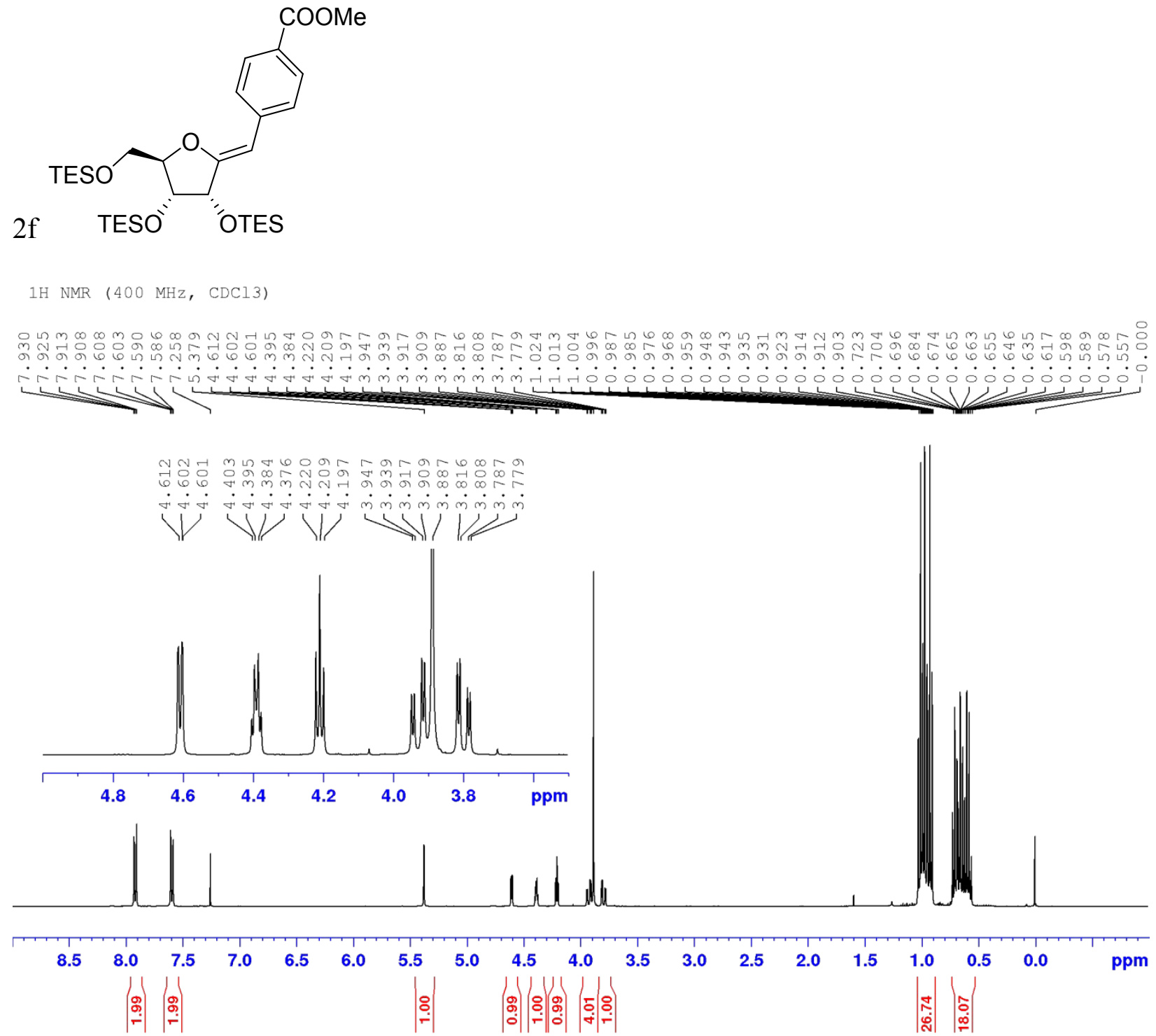

$13 \mathrm{C}\{1 \mathrm{H}\} \mathrm{NMR}(100 \mathrm{MHz}, \mathrm{CDCl} 3)$
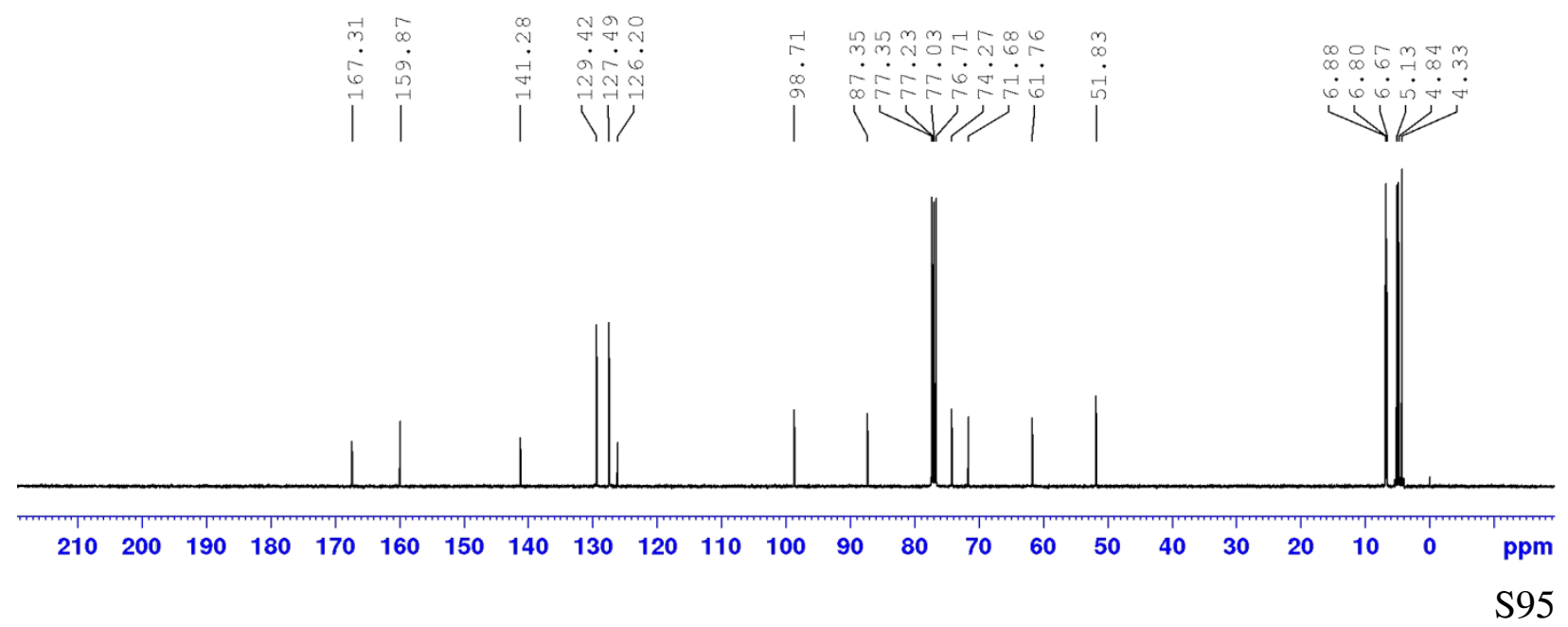


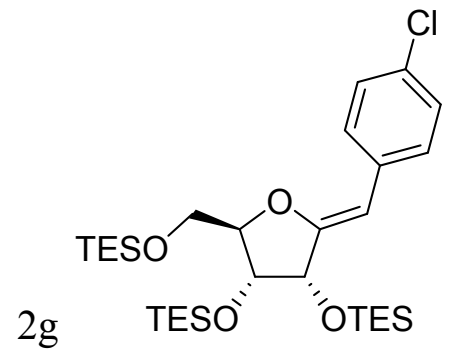

1H NMR (400 MHz, CDCl3)

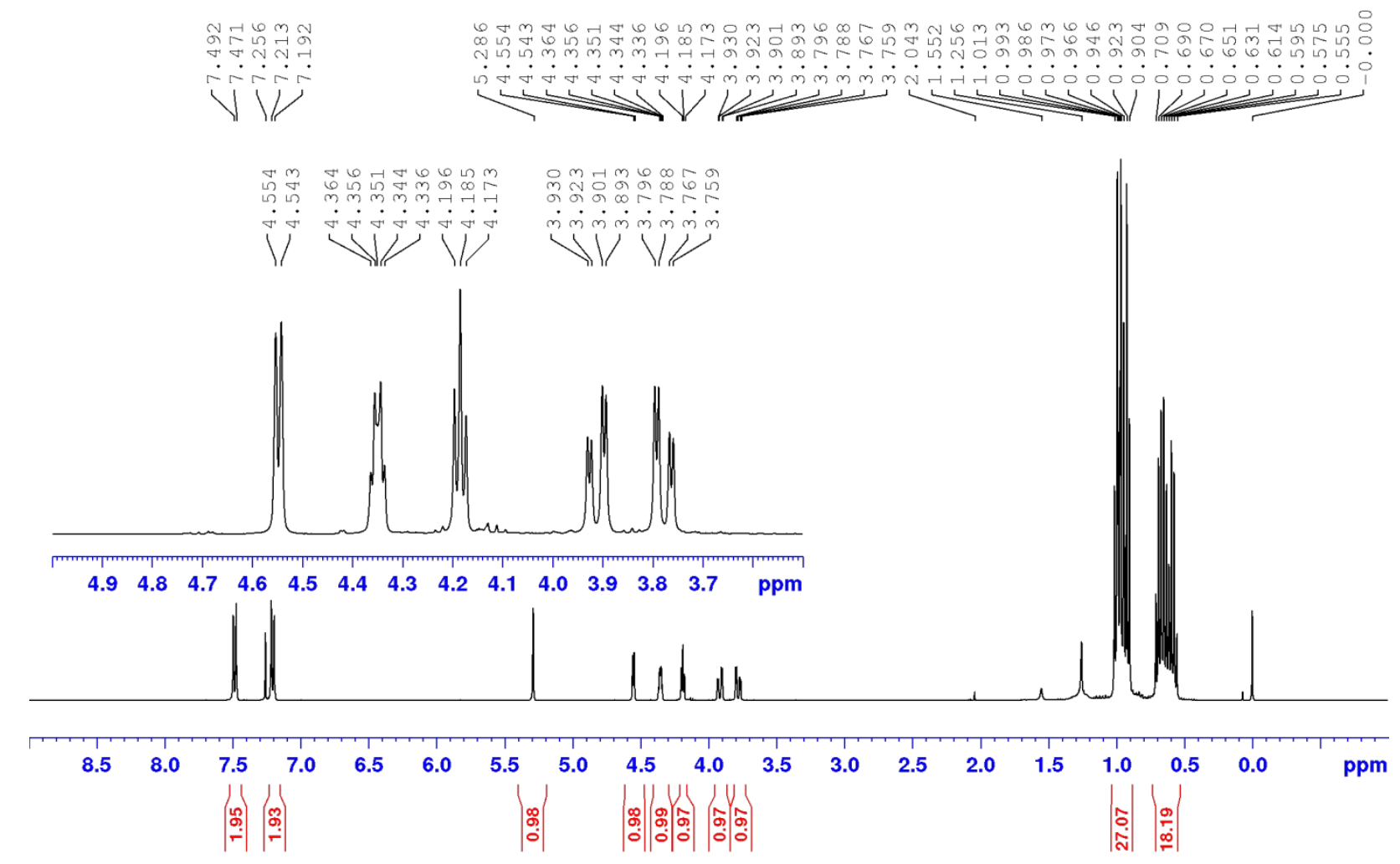




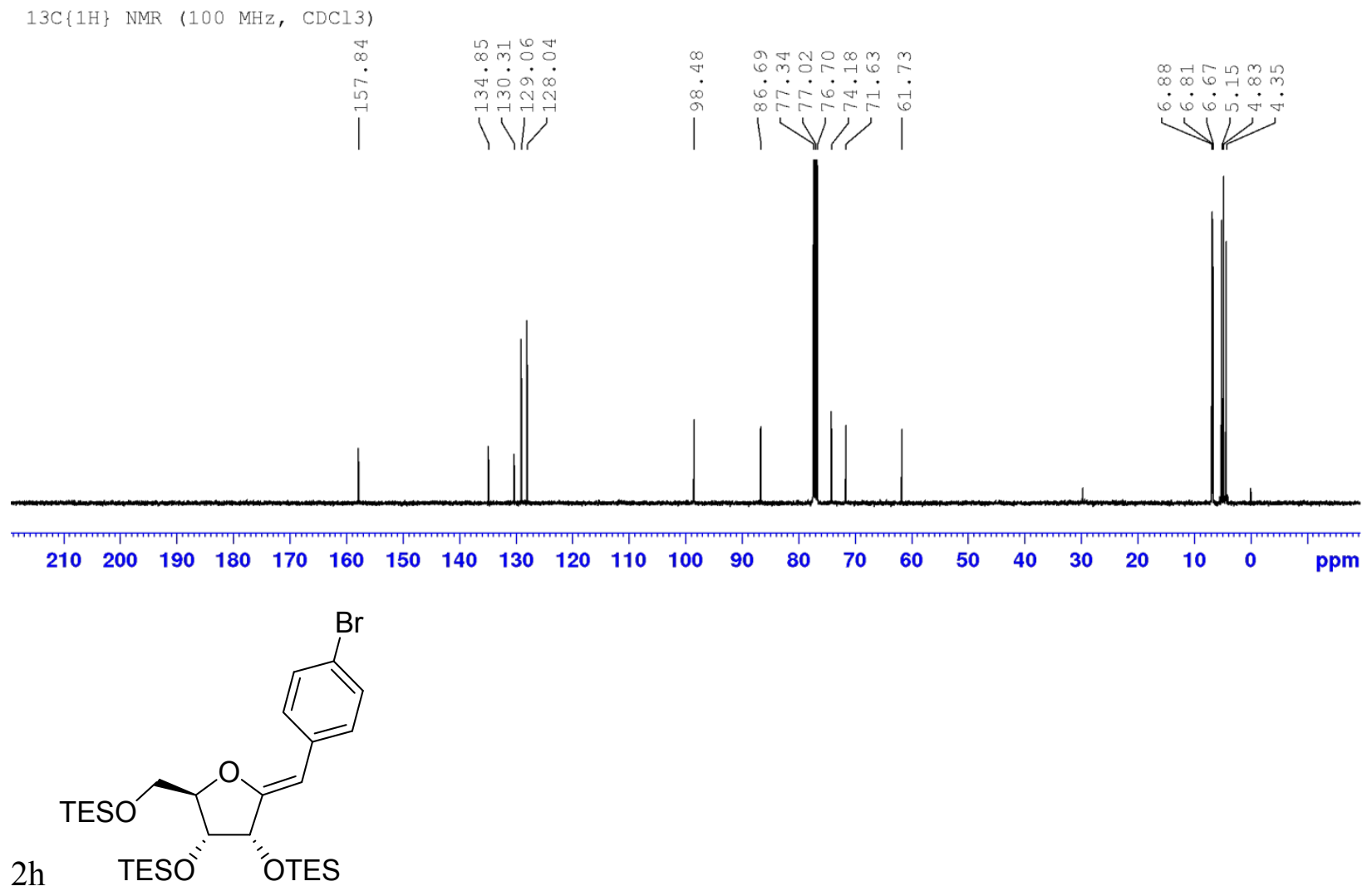


1H NMR (400 MHz, CDCl3)

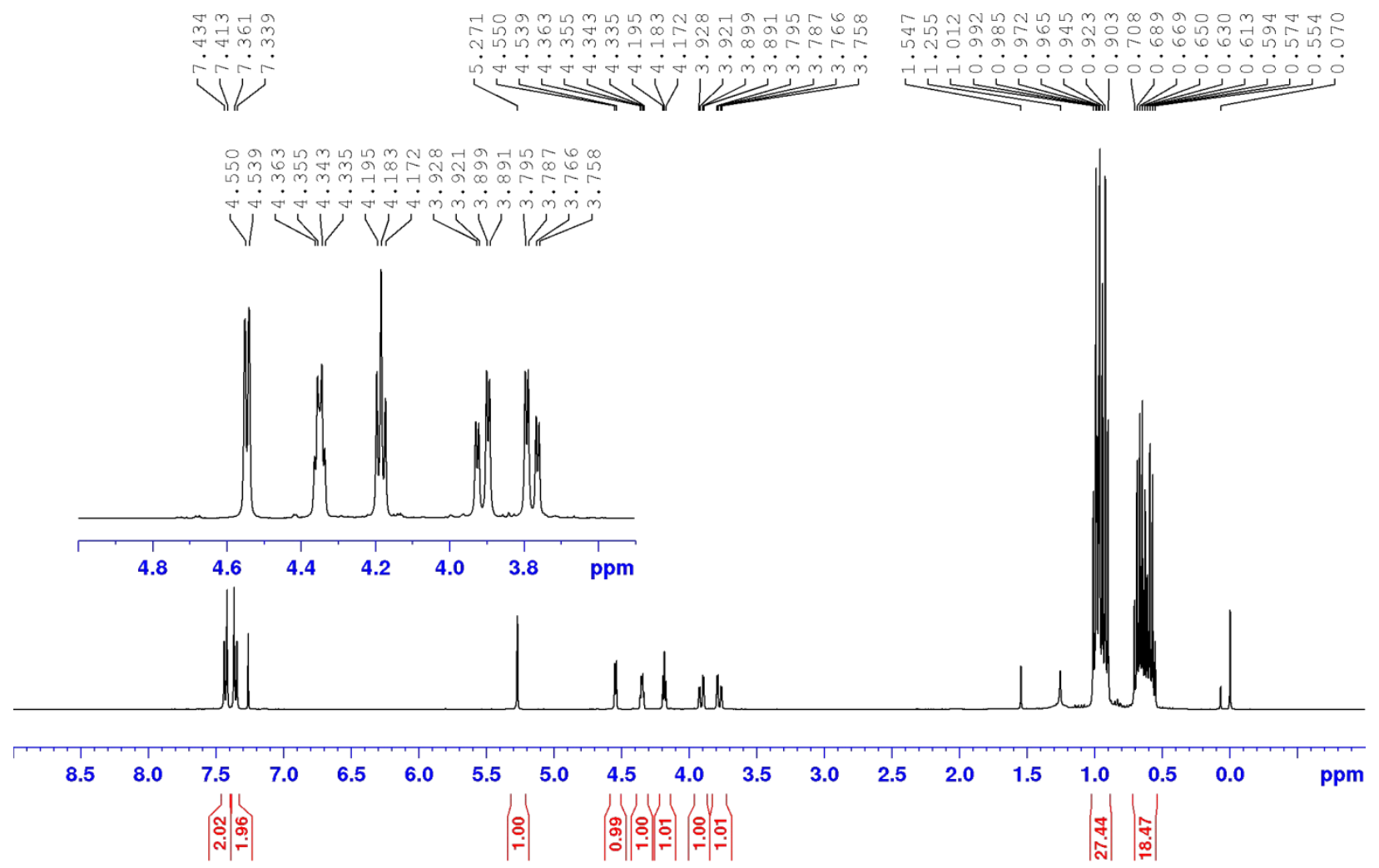

$13 \mathrm{C}\{1 \mathrm{H}\} \operatorname{NMR}(100 \mathrm{MHz}, \mathrm{CDCl} 3)$

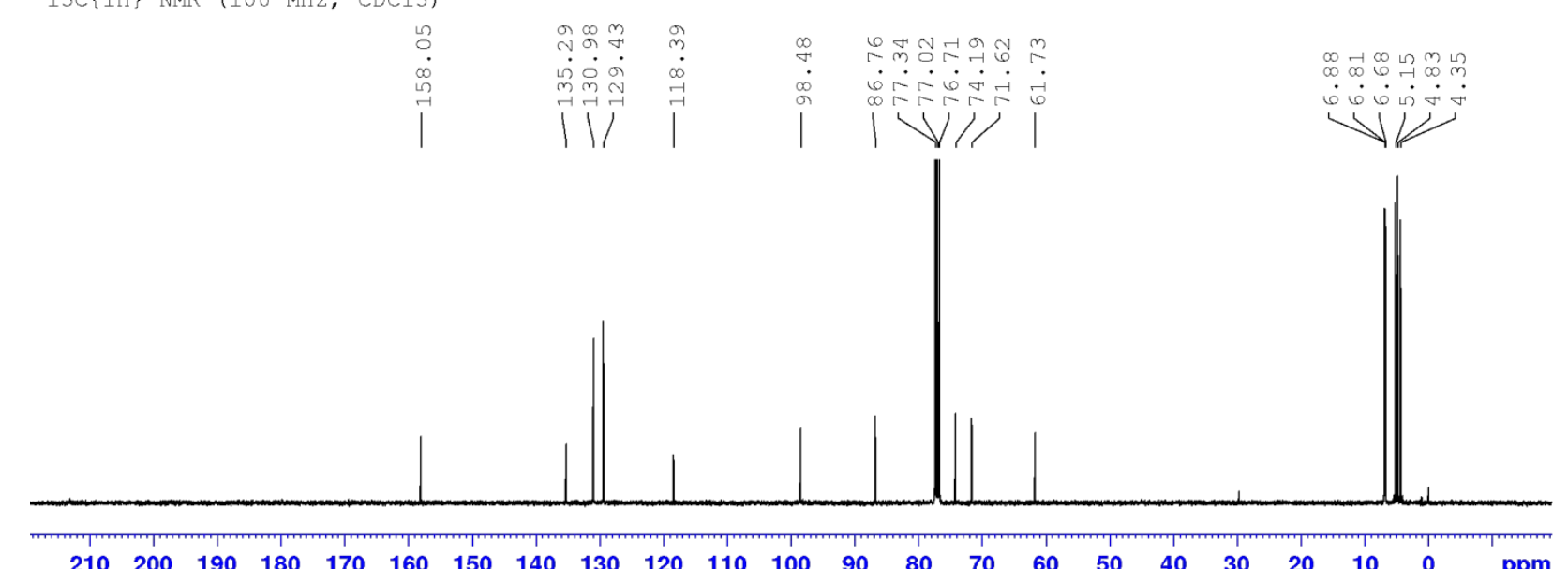

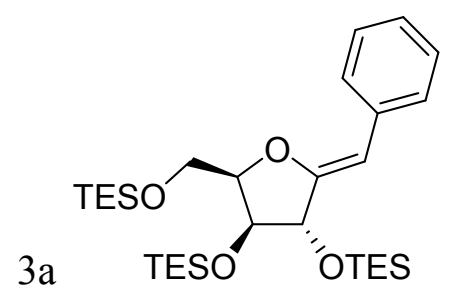




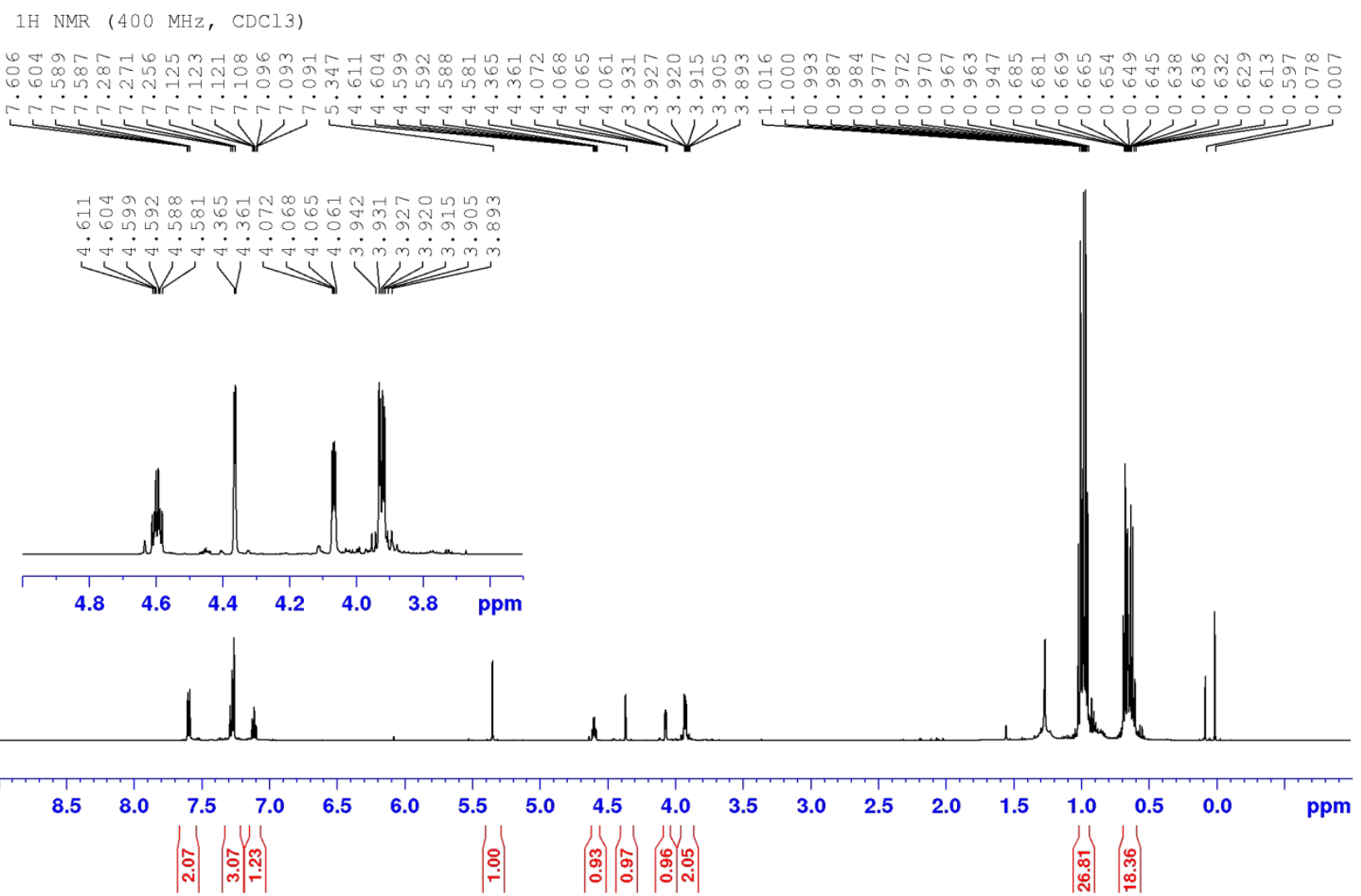

$13 \mathrm{C}\{1 \mathrm{H}\} \mathrm{NMR}(100 \mathrm{MHz}, \mathrm{CDCl} 3)$
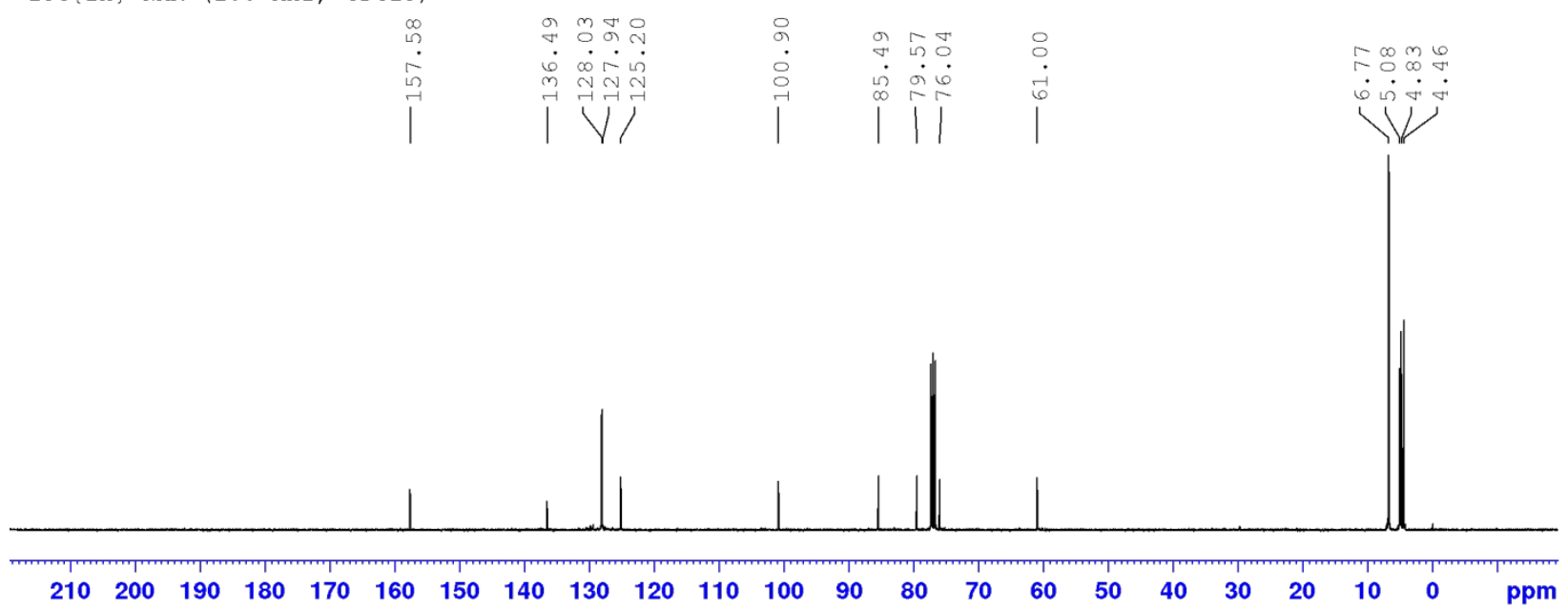


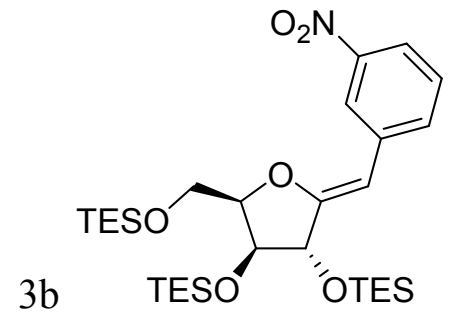

1H NMR (400 MHz, CDCl3)
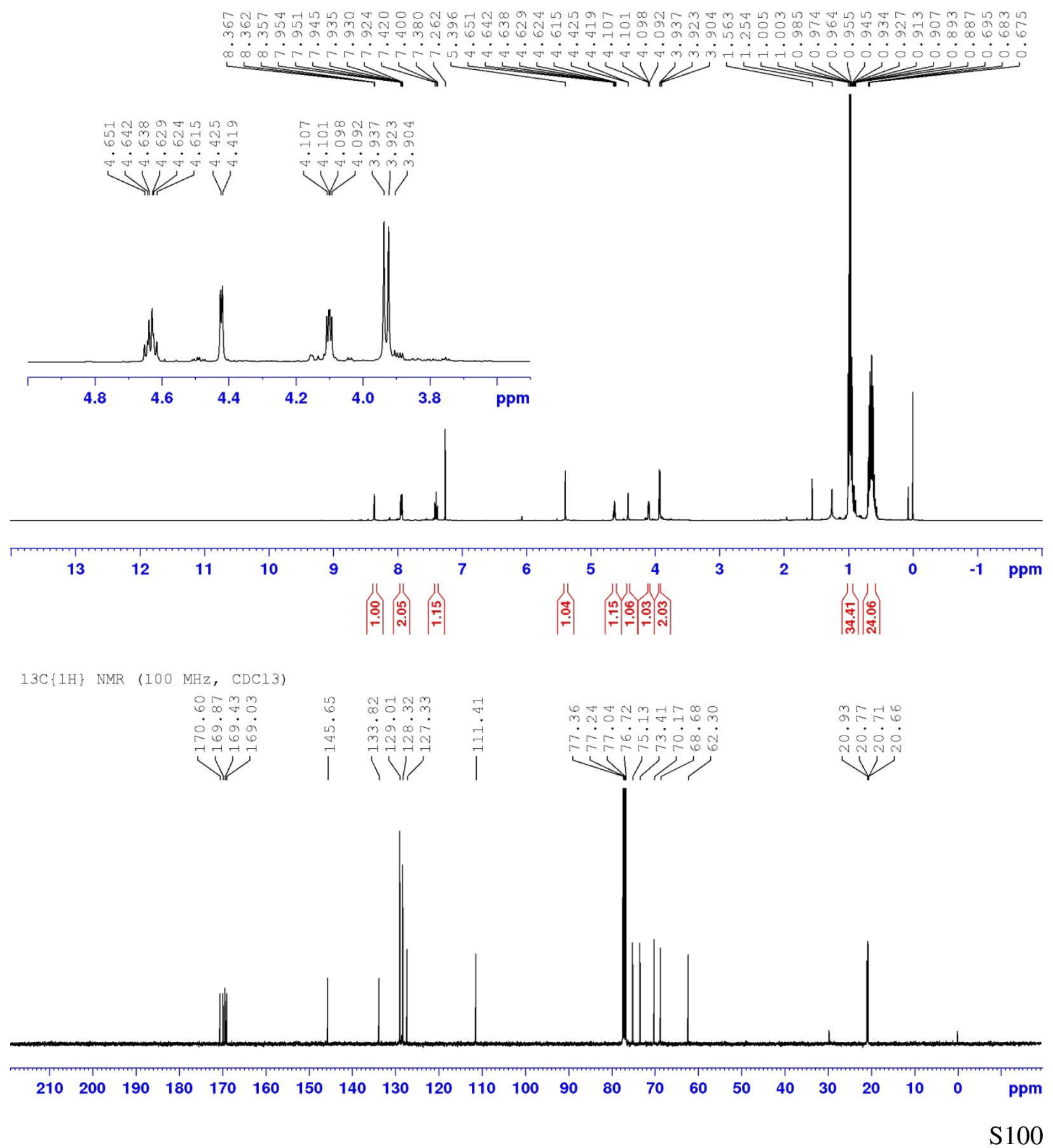


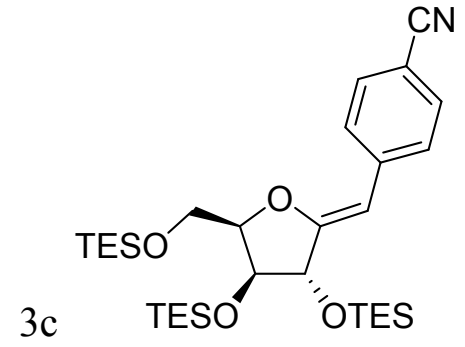

1H NMR (400 MHz, CDCl3)
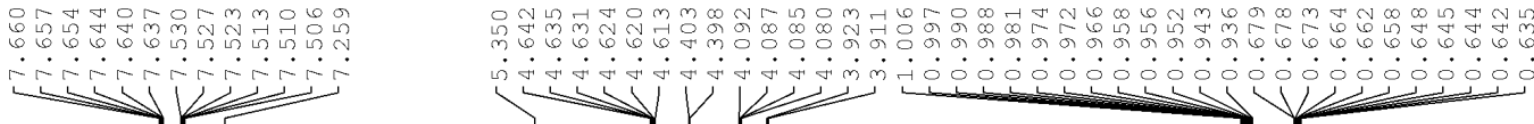

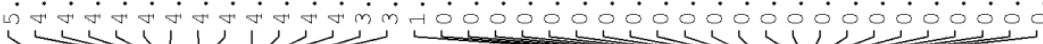

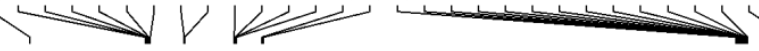

तथ

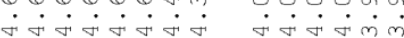

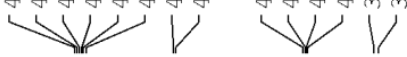
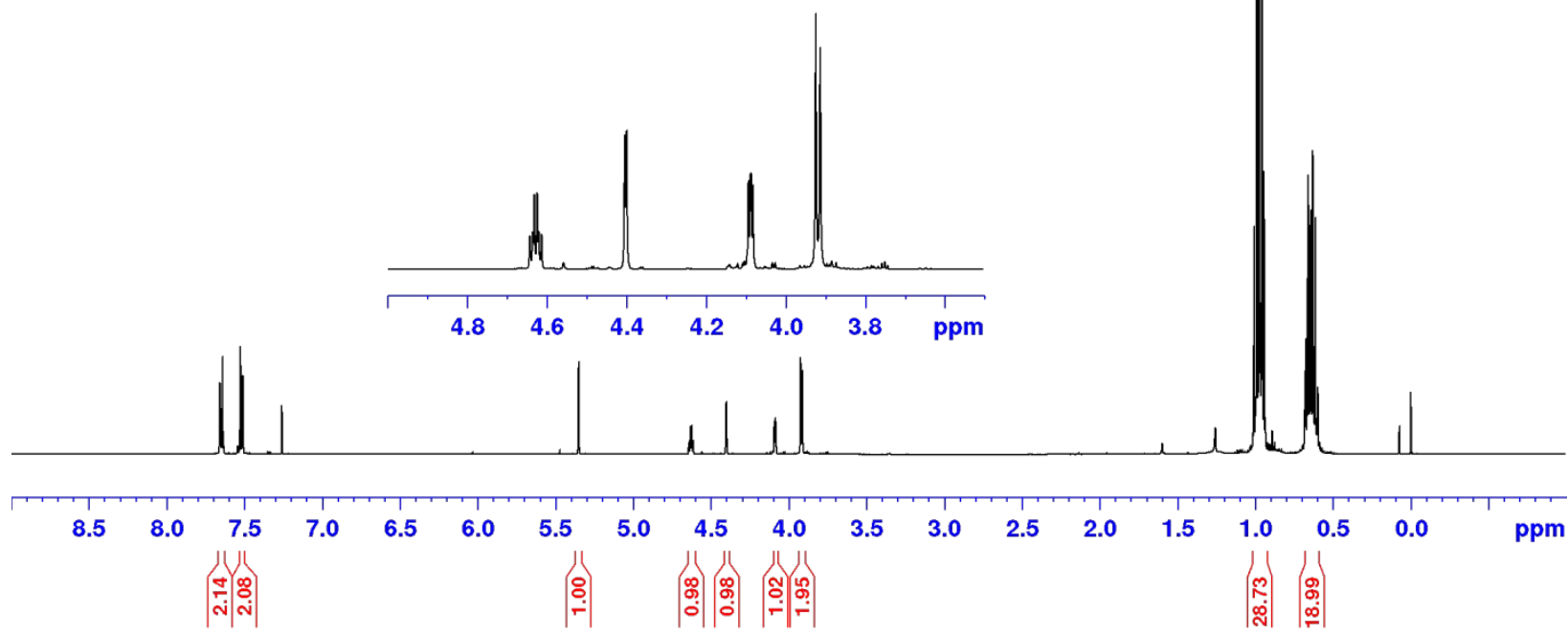

$13 \mathrm{C}\{1 \mathrm{H}\} \operatorname{NMR}(100 \mathrm{MHz}, \mathrm{CDCl} 3)$
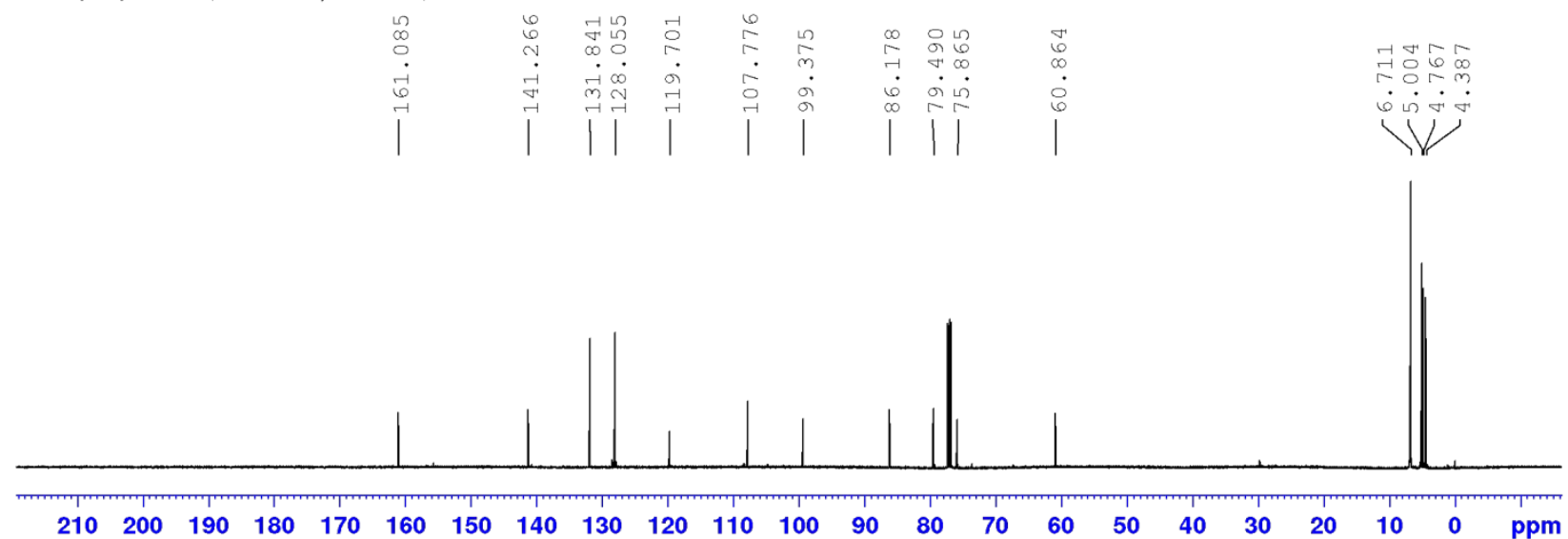


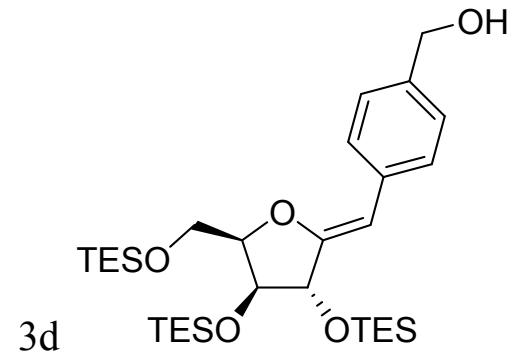

1H NMR (400 MHz, CDCl3)
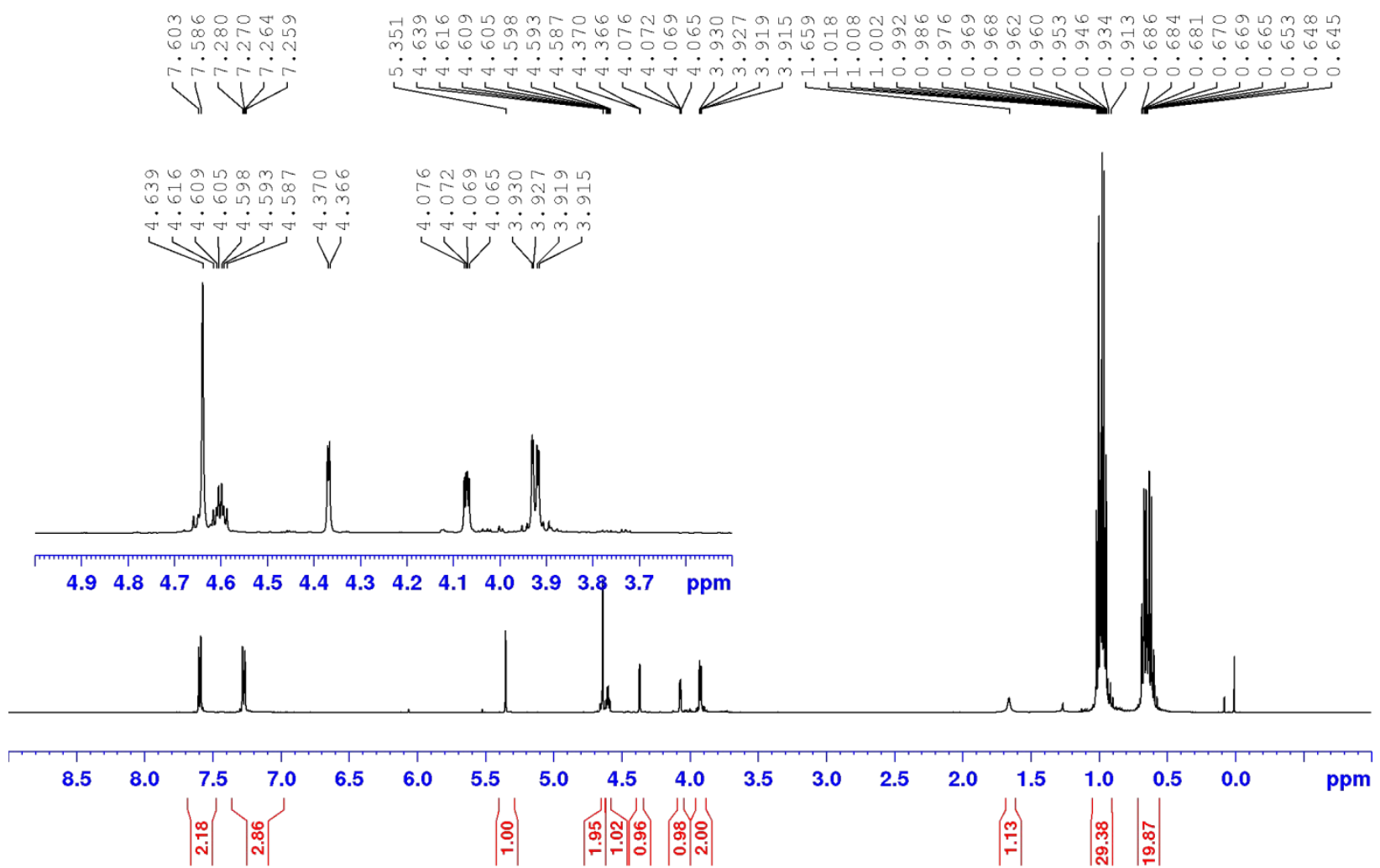


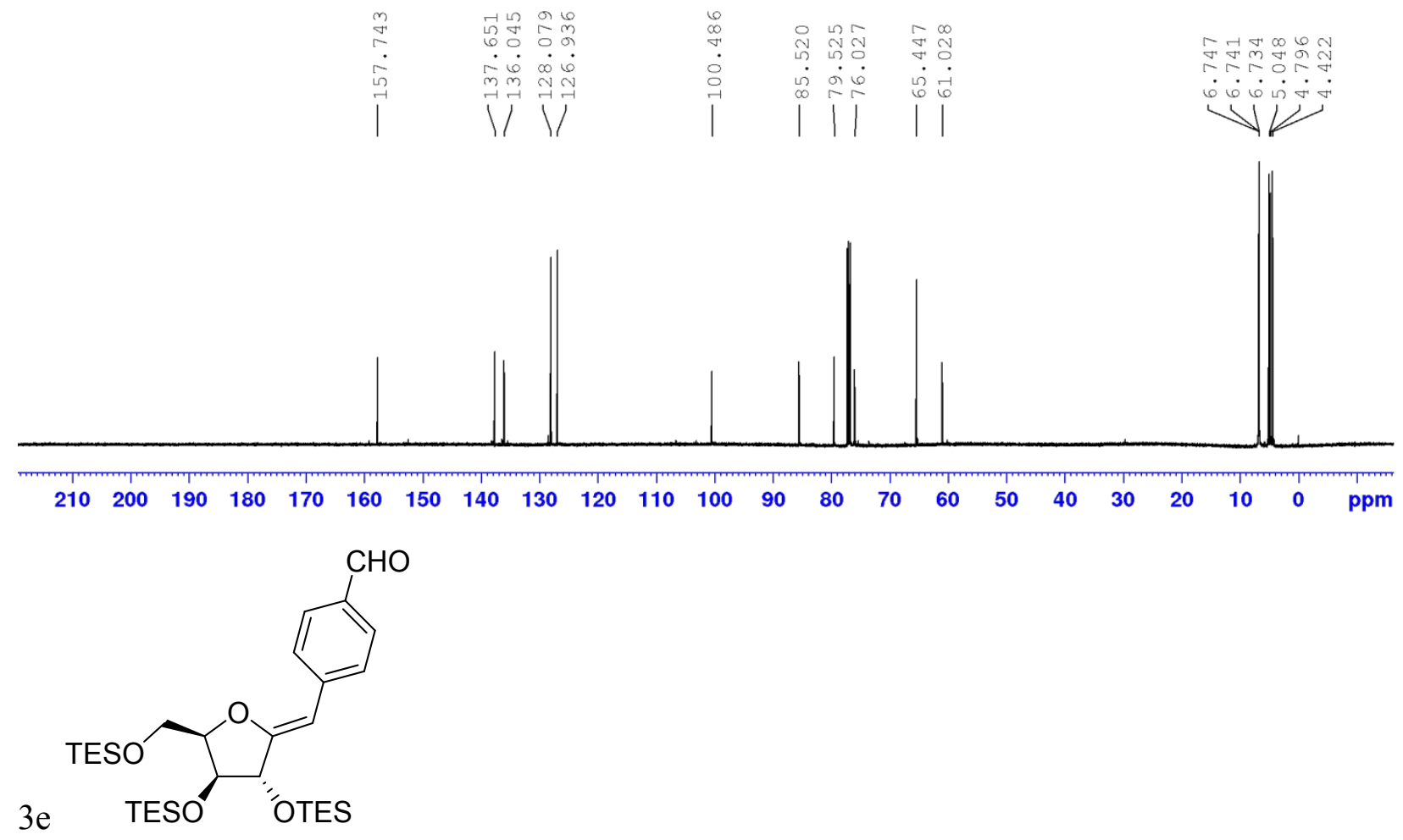

$1 \mathrm{H}$ NMR (400 MHz, CDCl3)
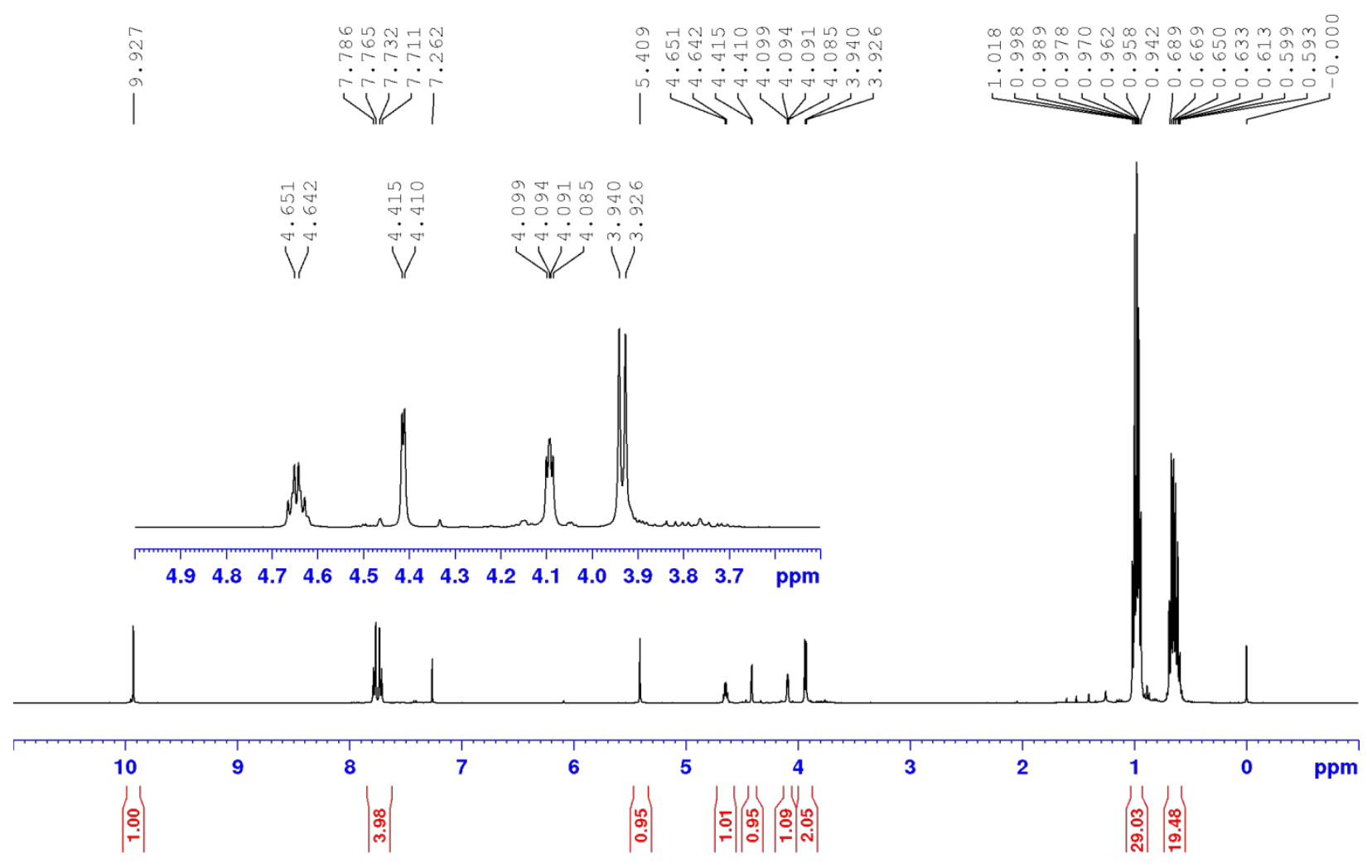


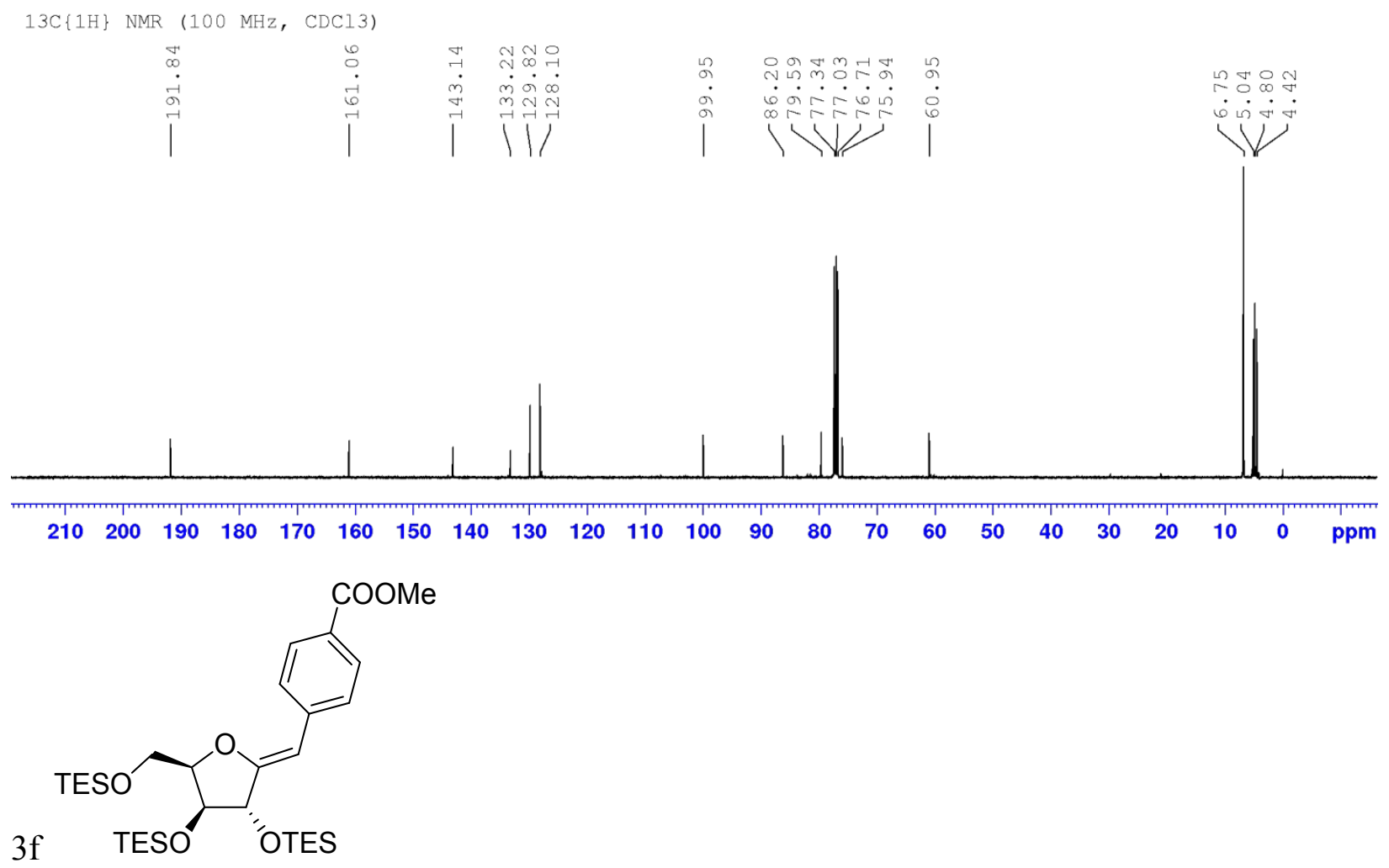

1H NMR ( $400 \mathrm{MHz}, \operatorname{CDCl} 3)$

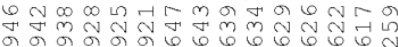

न

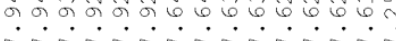

m ॠु

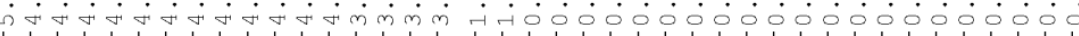

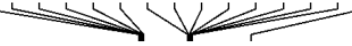

(1)

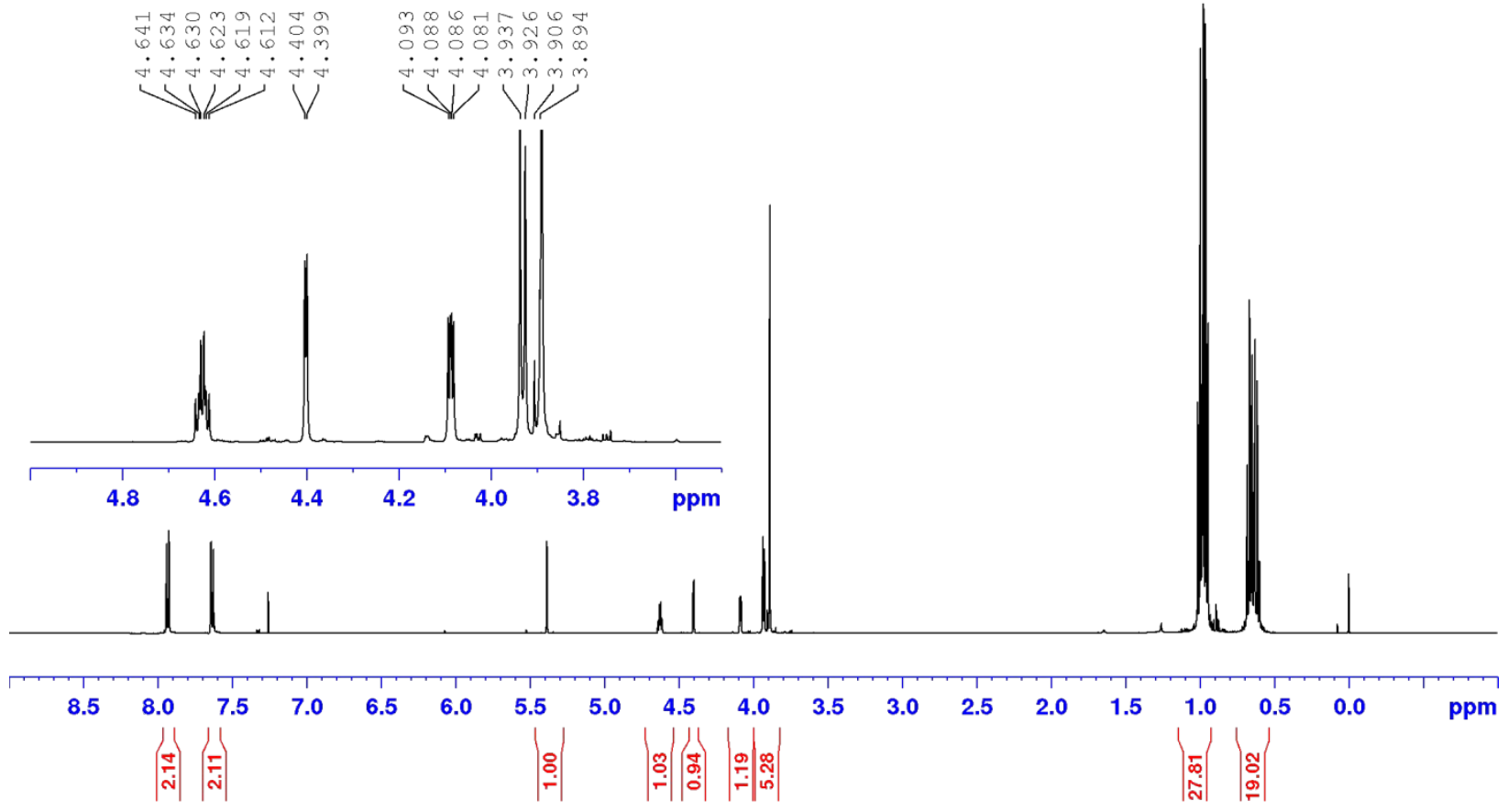




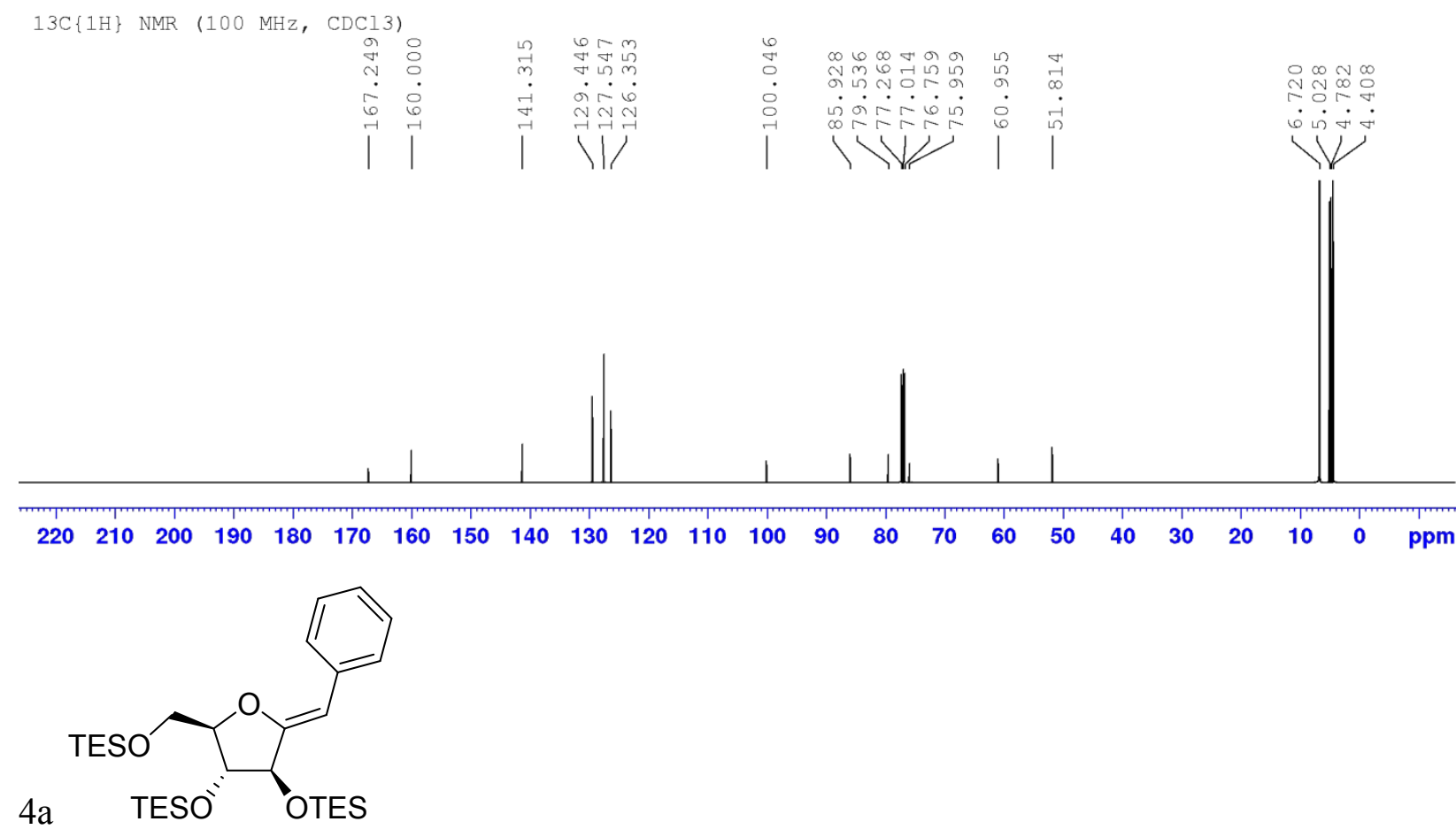

$1 \mathrm{H}$ NMR (400 $\mathrm{MHz}, \mathrm{CDCl} 3)$

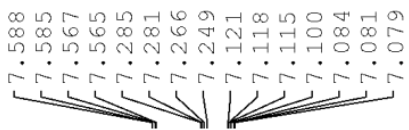

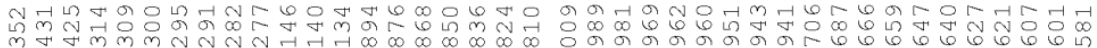

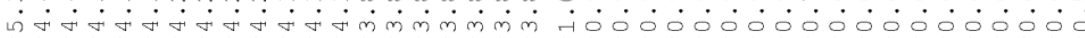

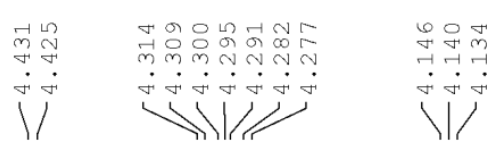

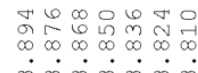

11111
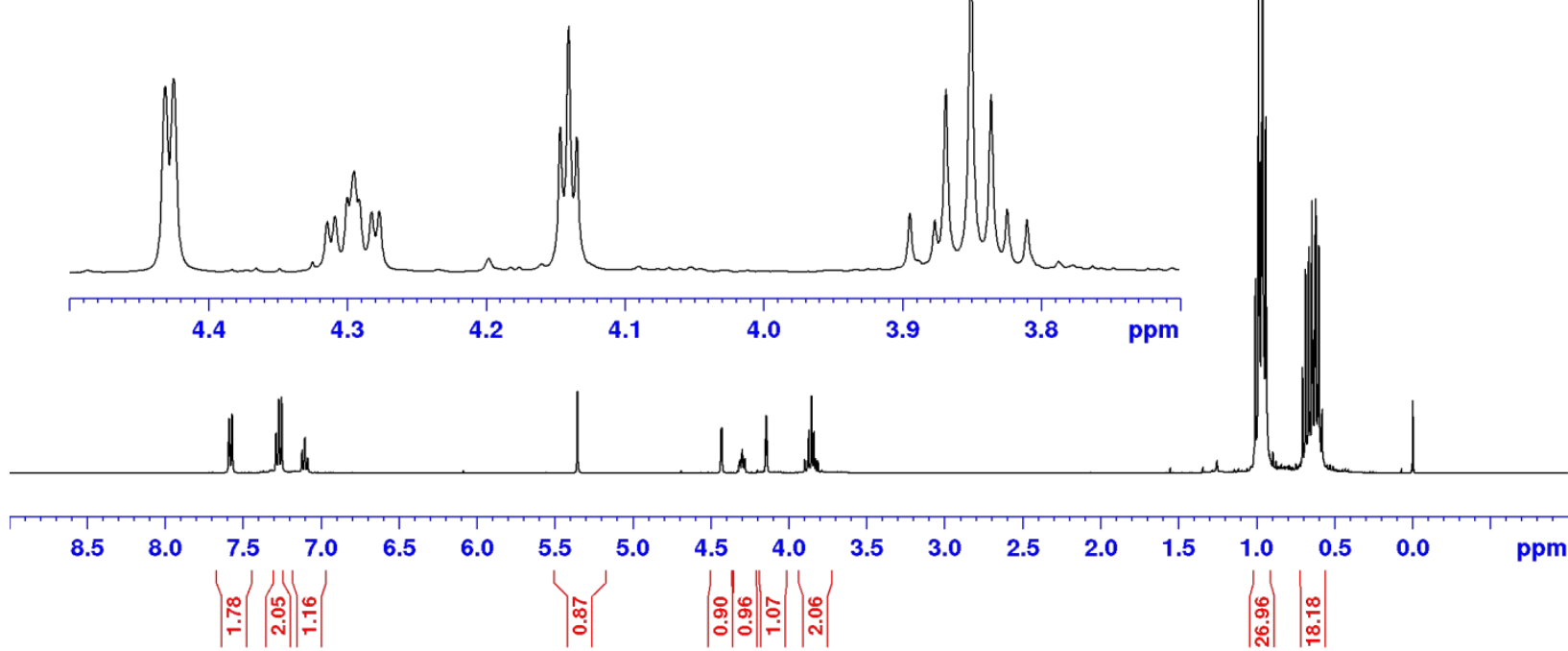


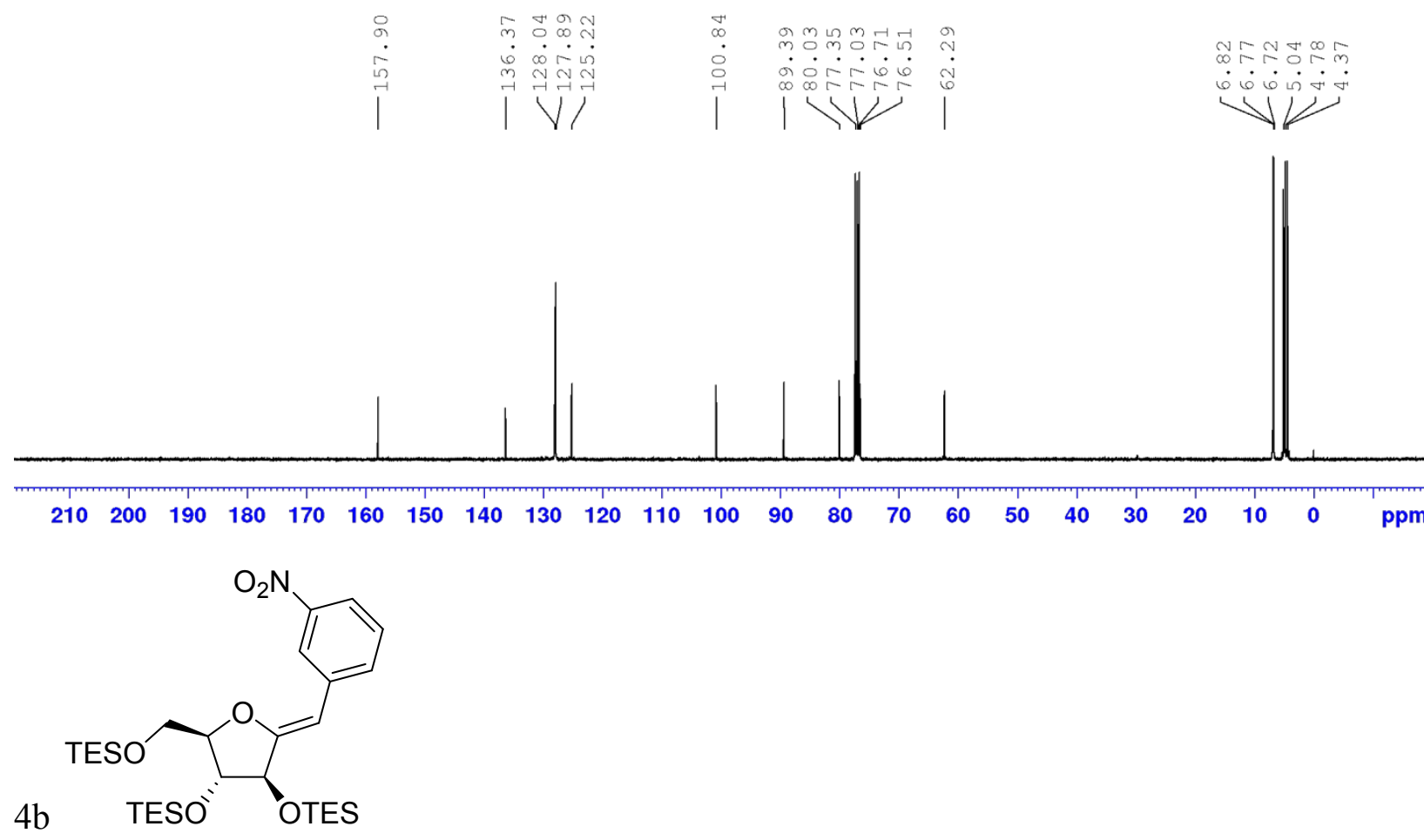

1H NMR (400 MHz, CDCl3)

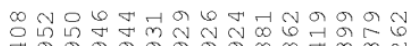

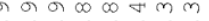

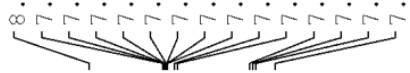

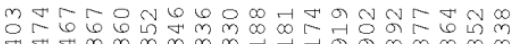

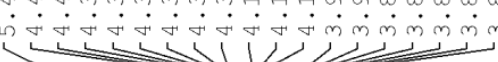

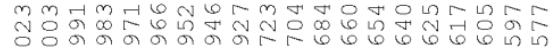

-

(

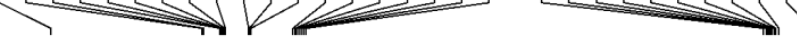

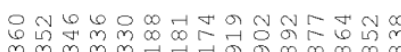

$\dot{0} \dot{0} \dot{0} \dot{0} \cdot \dot{0} \cdot \dot{0}$

(litis
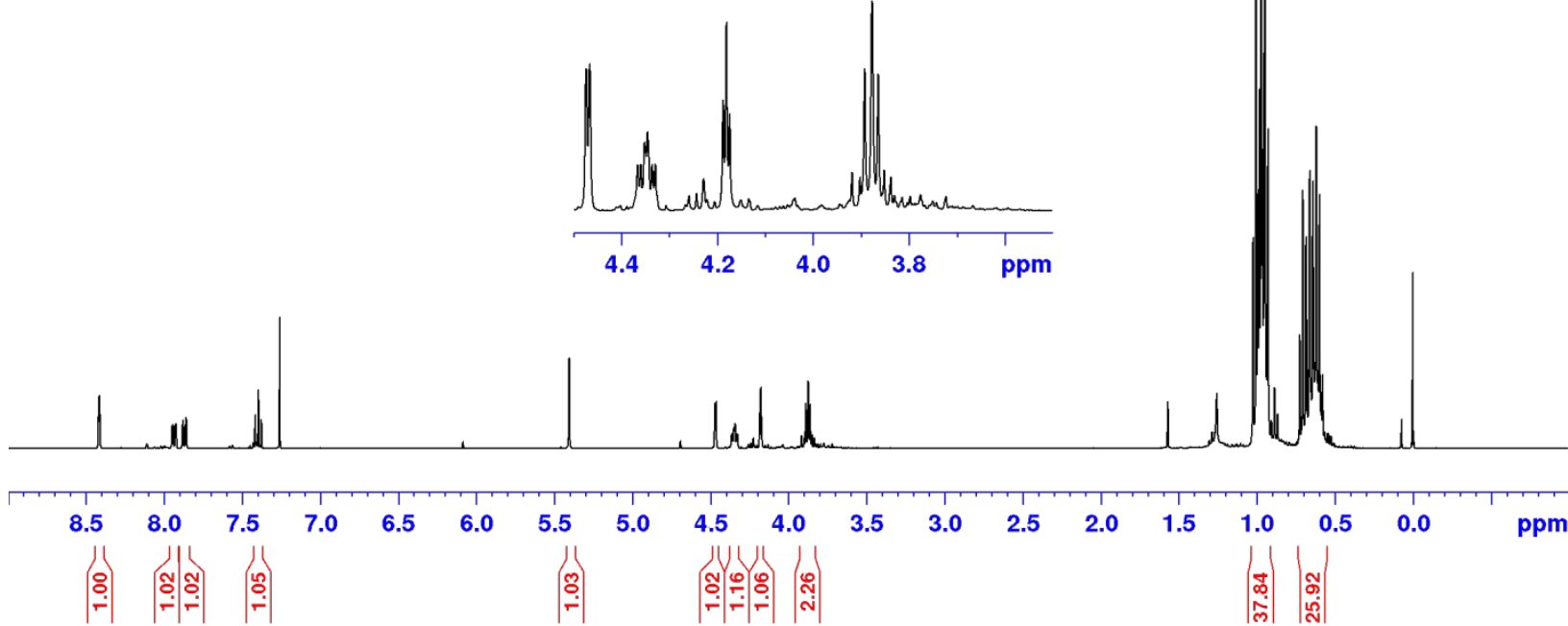

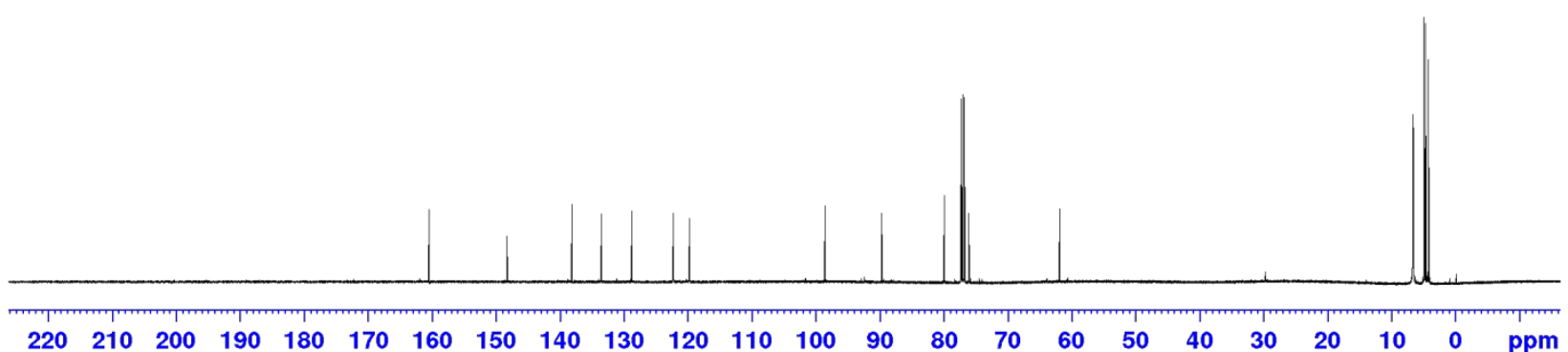<smiles>CO[C@@H]1[C@H](OC#[SH])/C(=C/c2ccc(C#N)cc2)O[C@@H]1COC(F)(F)F</smiles>

1H NMR (400 MHz, $\mathrm{CDCl} 3)$

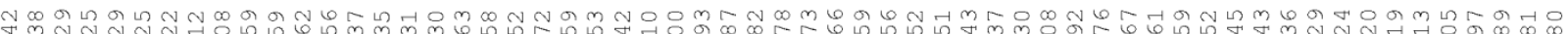

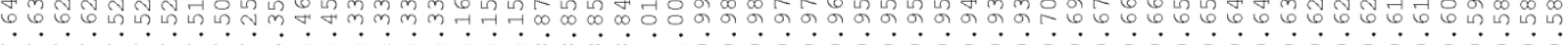

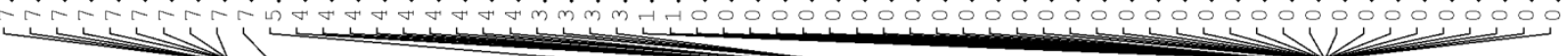
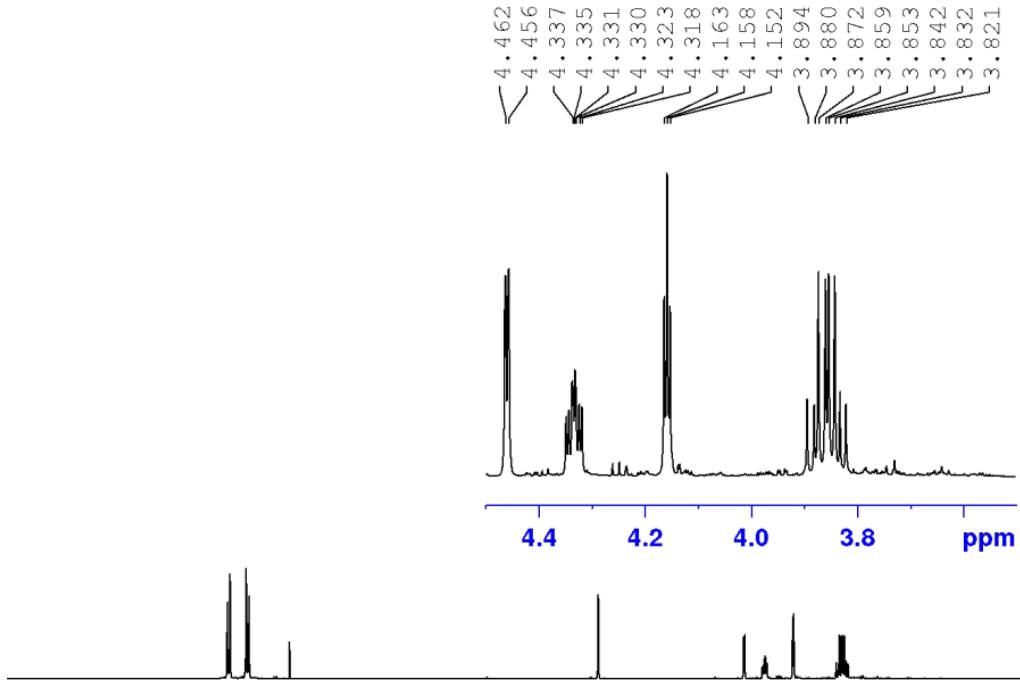

$\int$

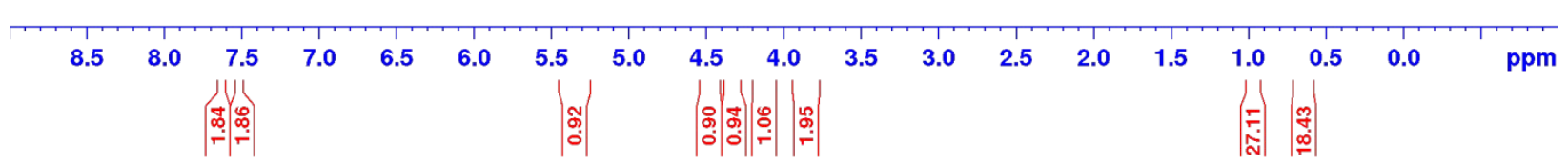



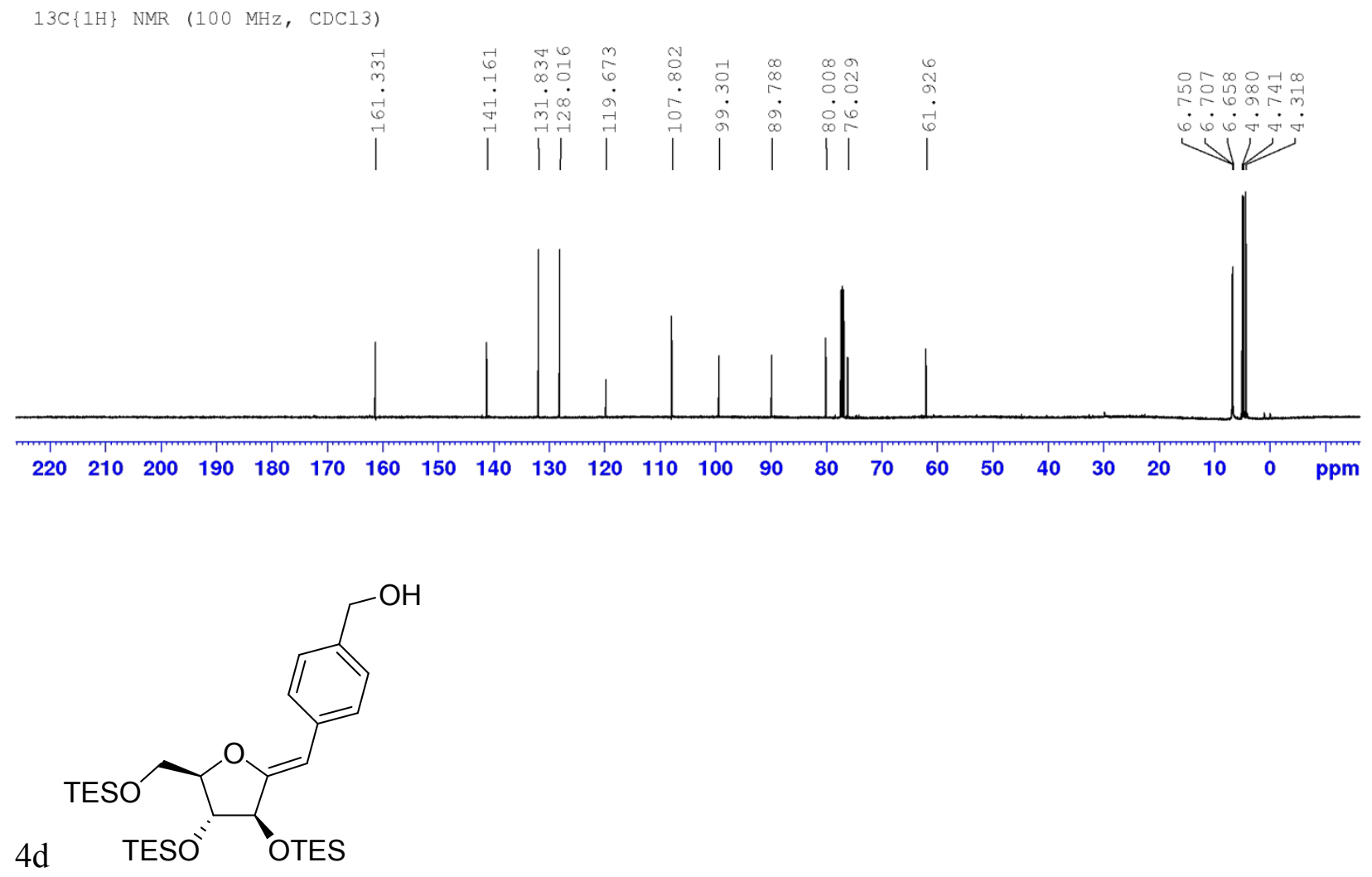

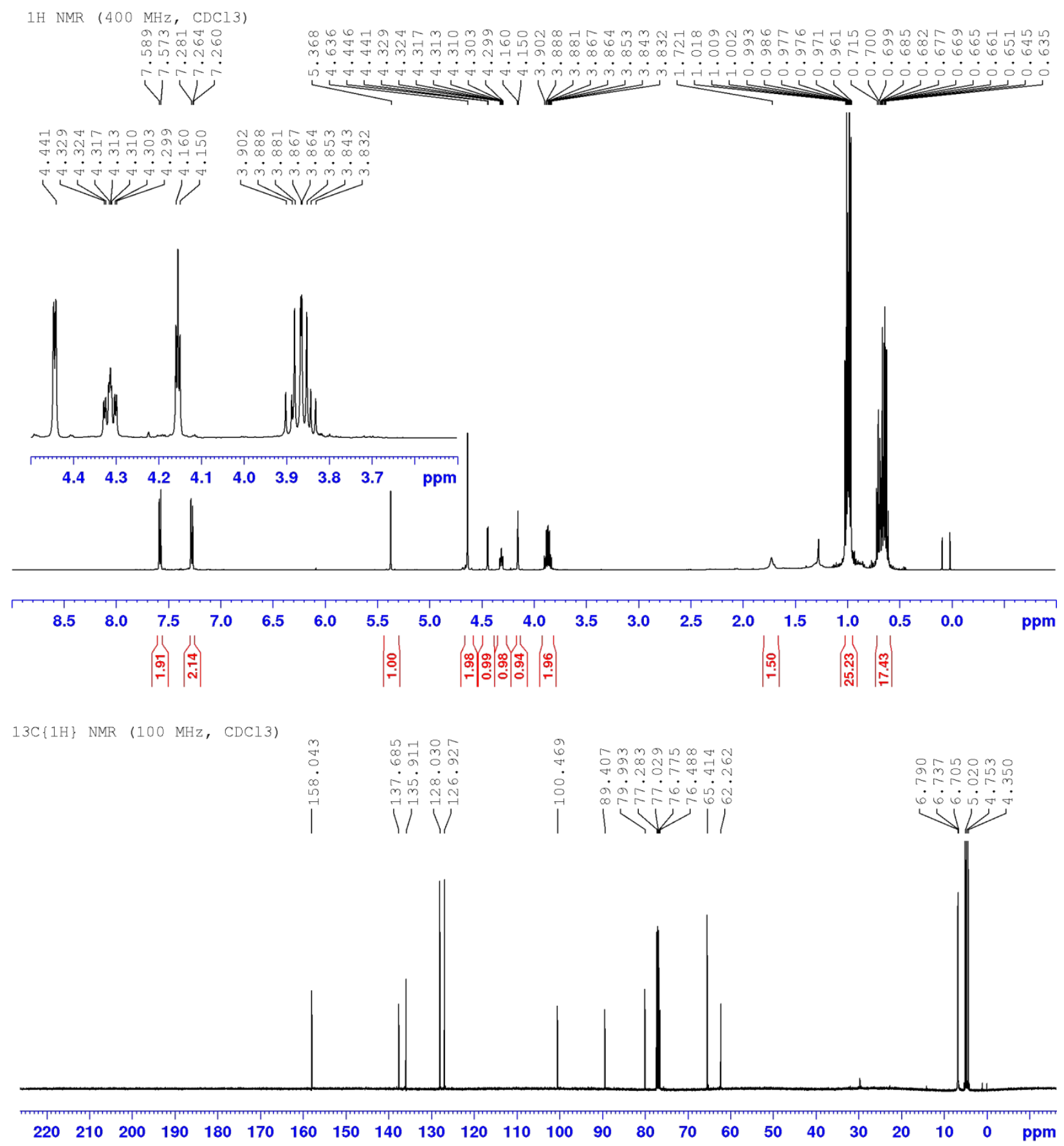


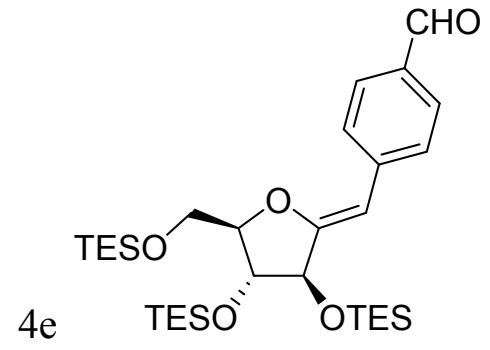

$1 \mathrm{H}$ NMR (400 MHz, CDCl3)
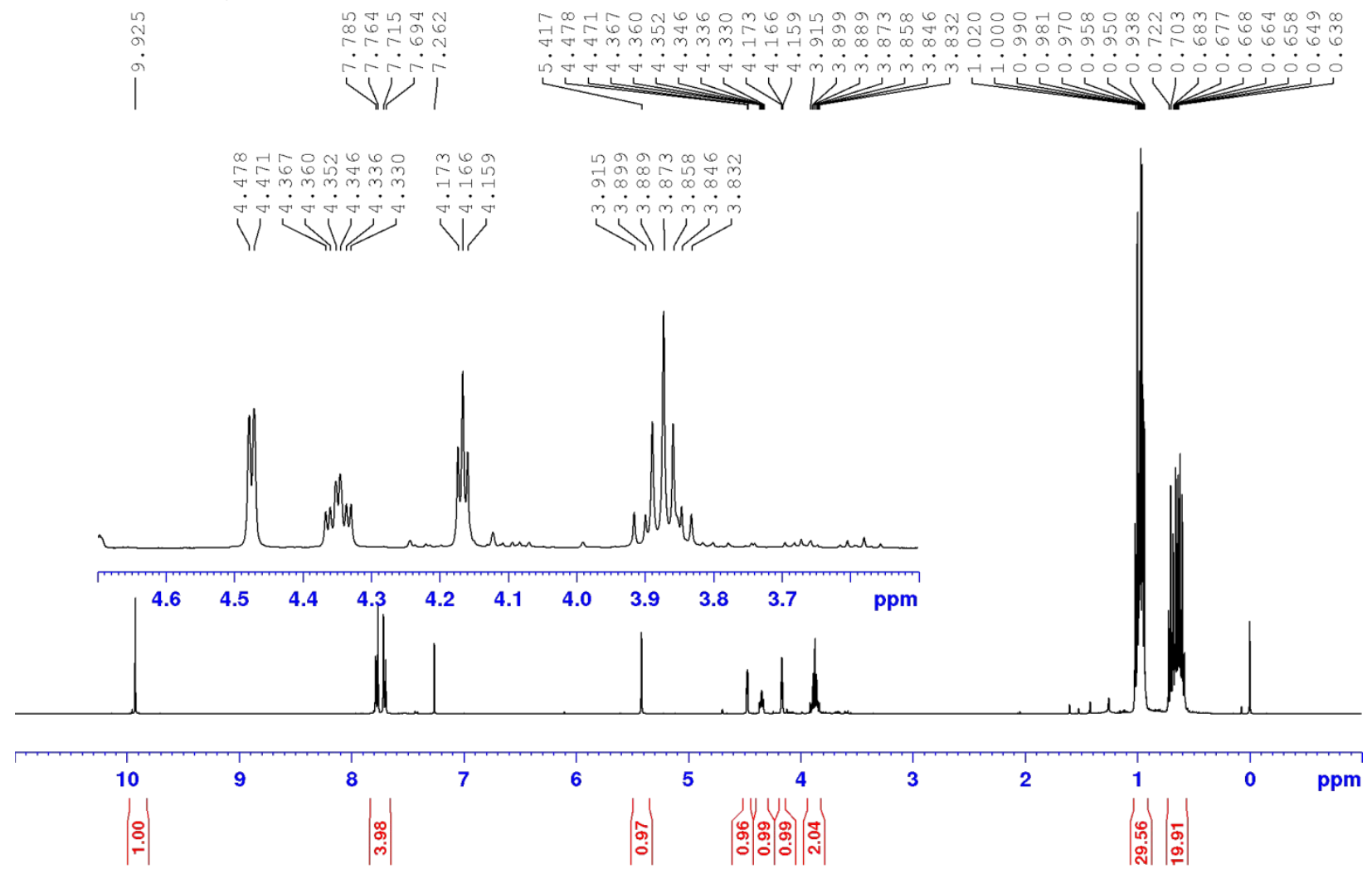

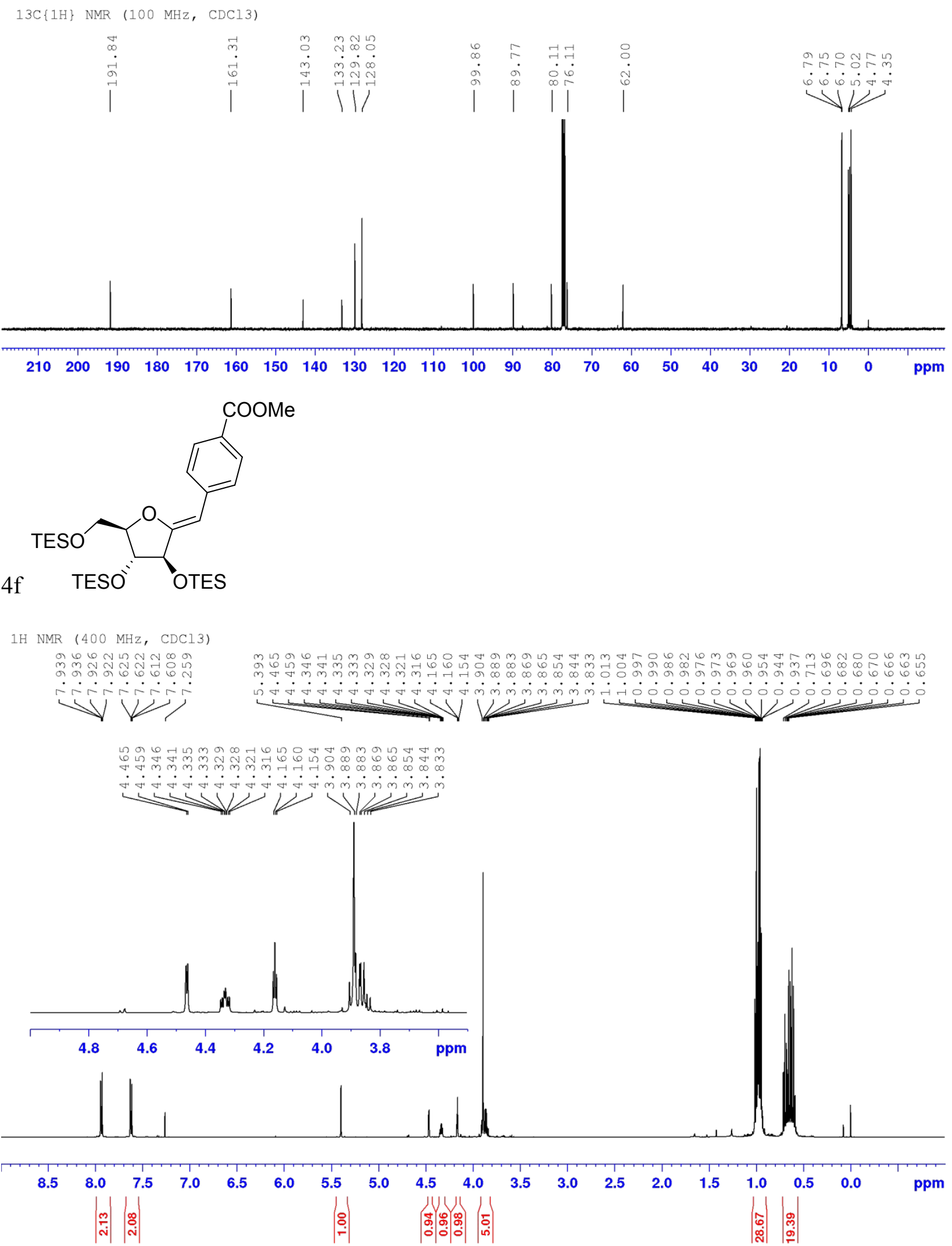


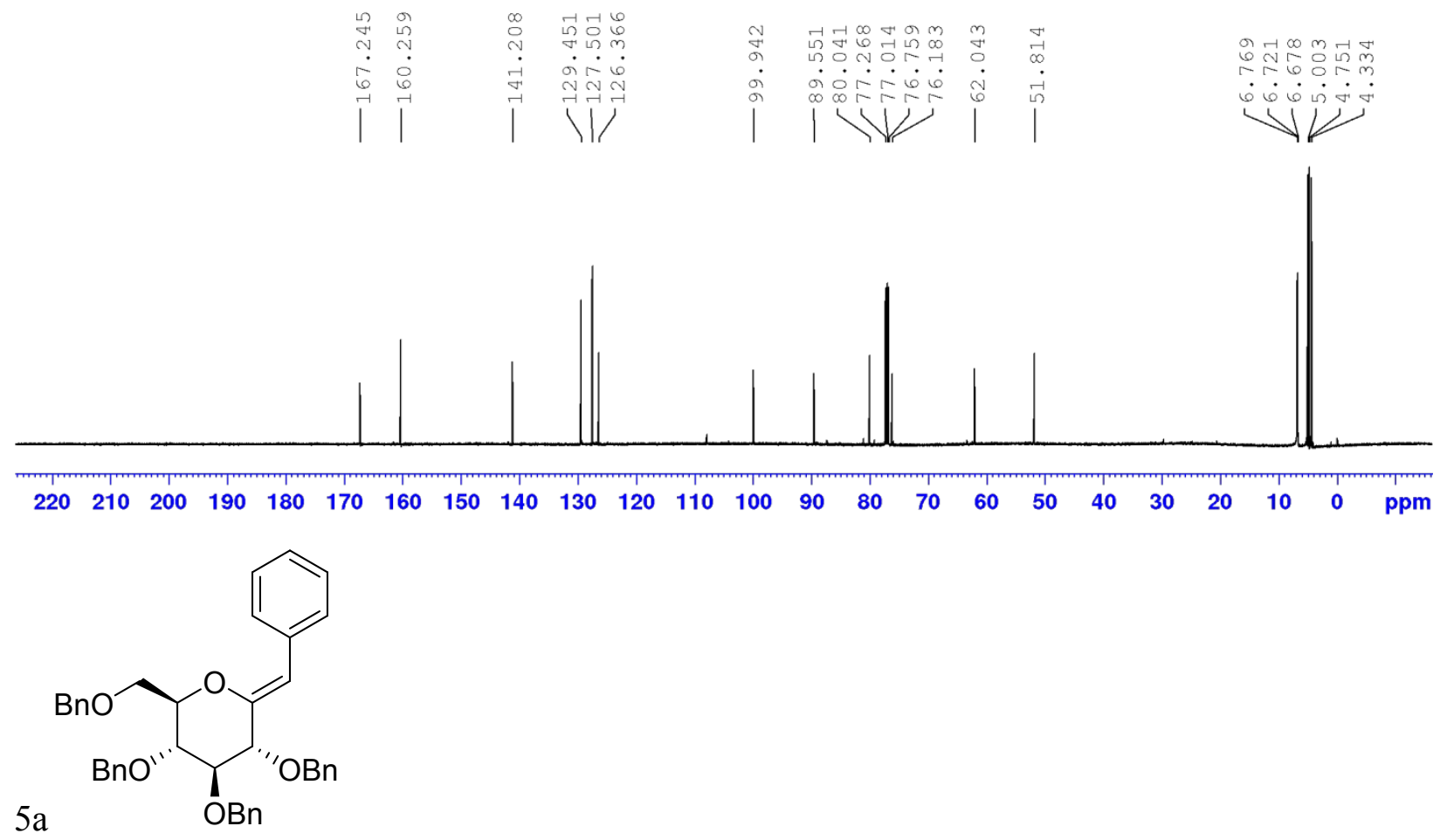




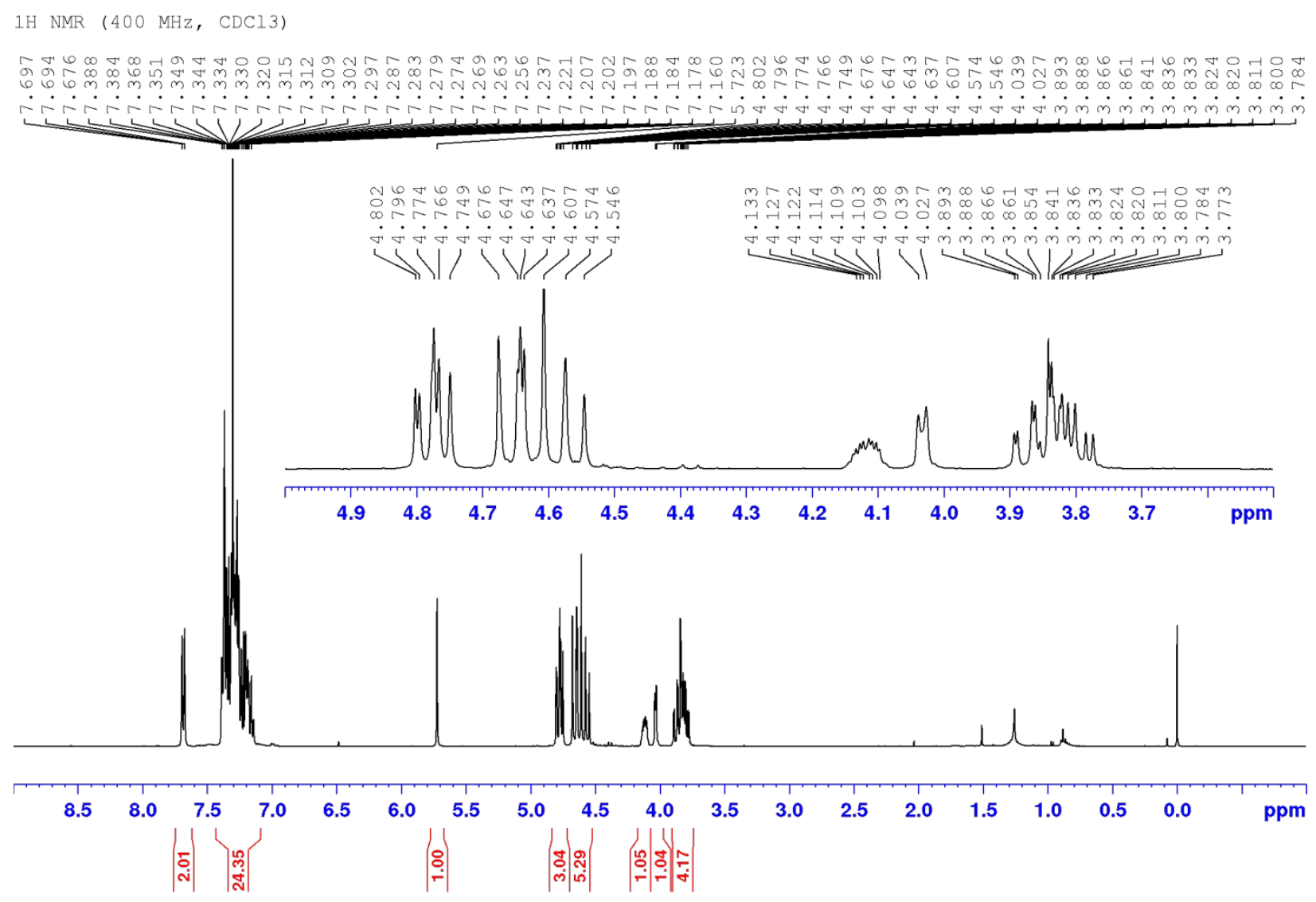

$13 \mathrm{C}\{1 \mathrm{H}\} \operatorname{NMR}(100 \mathrm{MHz}, \mathrm{CDCl} 3)$

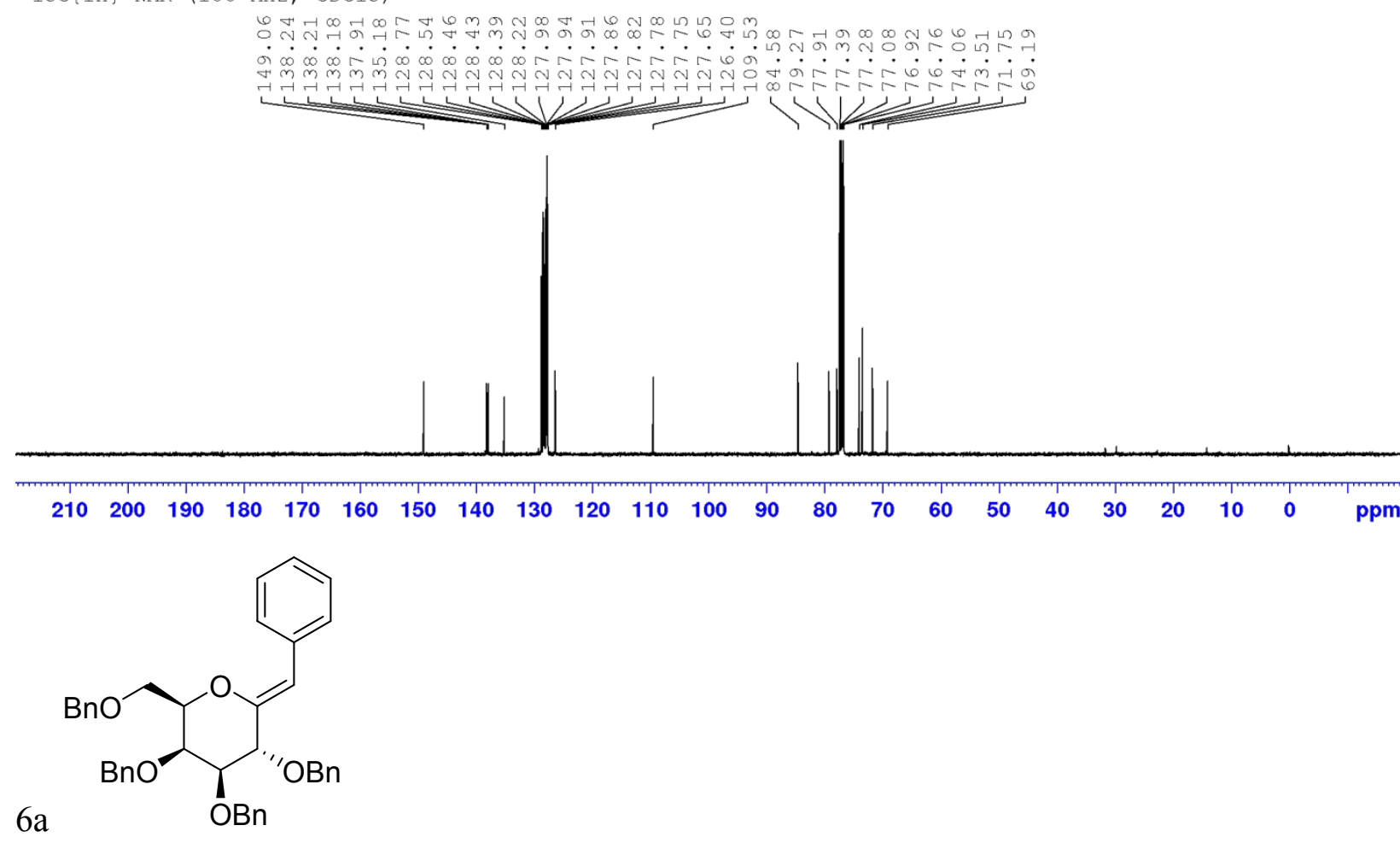



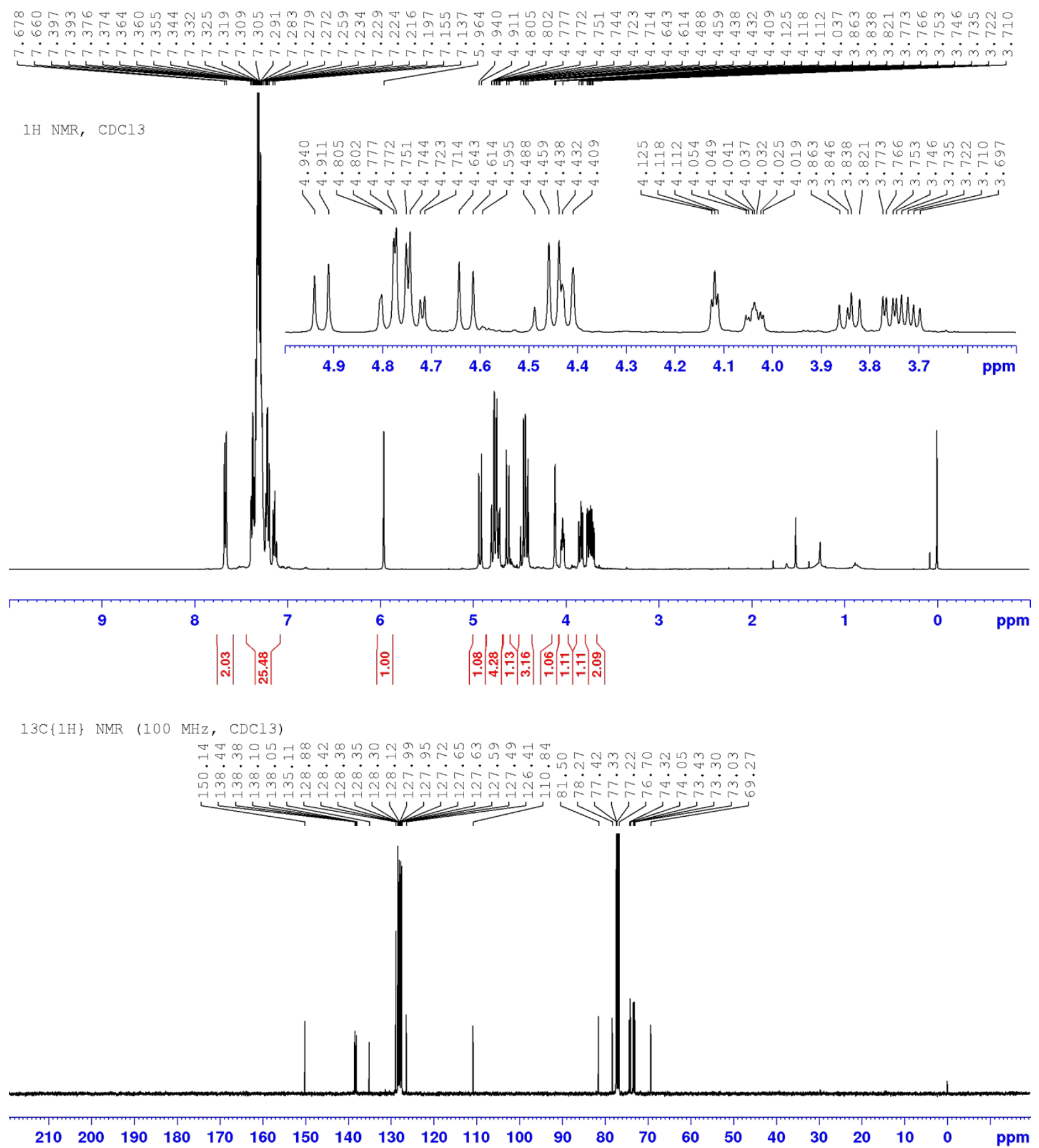
<smiles>OCCC(Br)(Br)c1ccccc1</smiles>

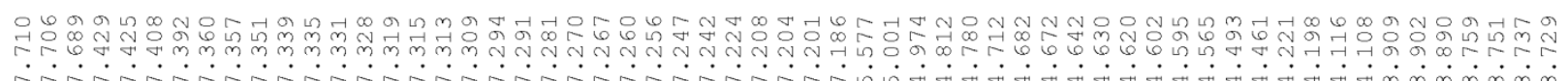

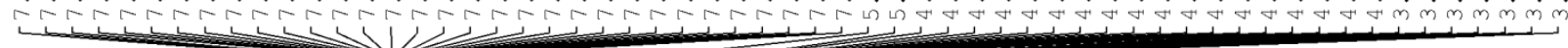

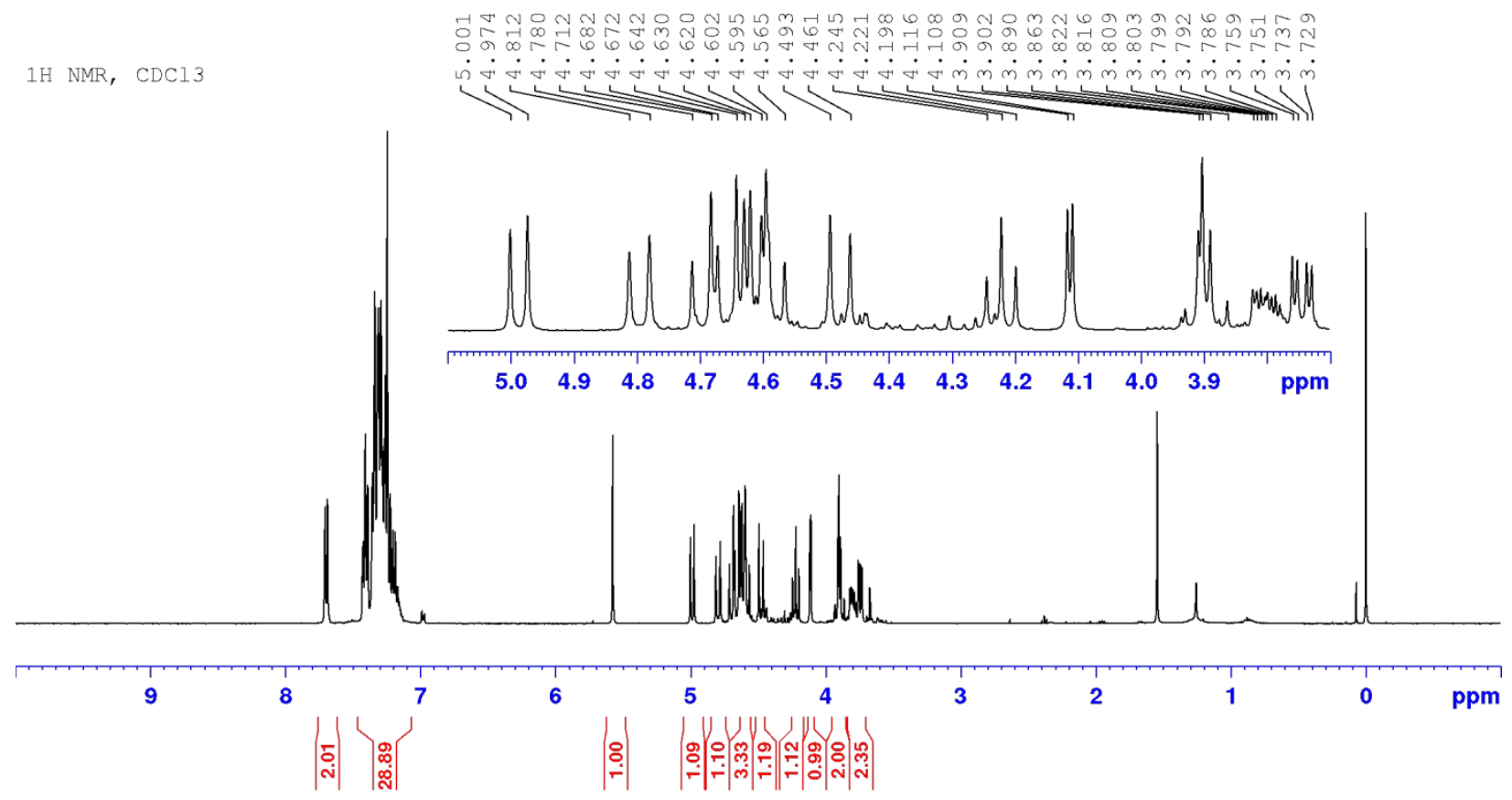

$13 \mathrm{C}\{1 \mathrm{H}\}$ NMR $(100 \mathrm{MHz}, \mathrm{CDCl} 3)$
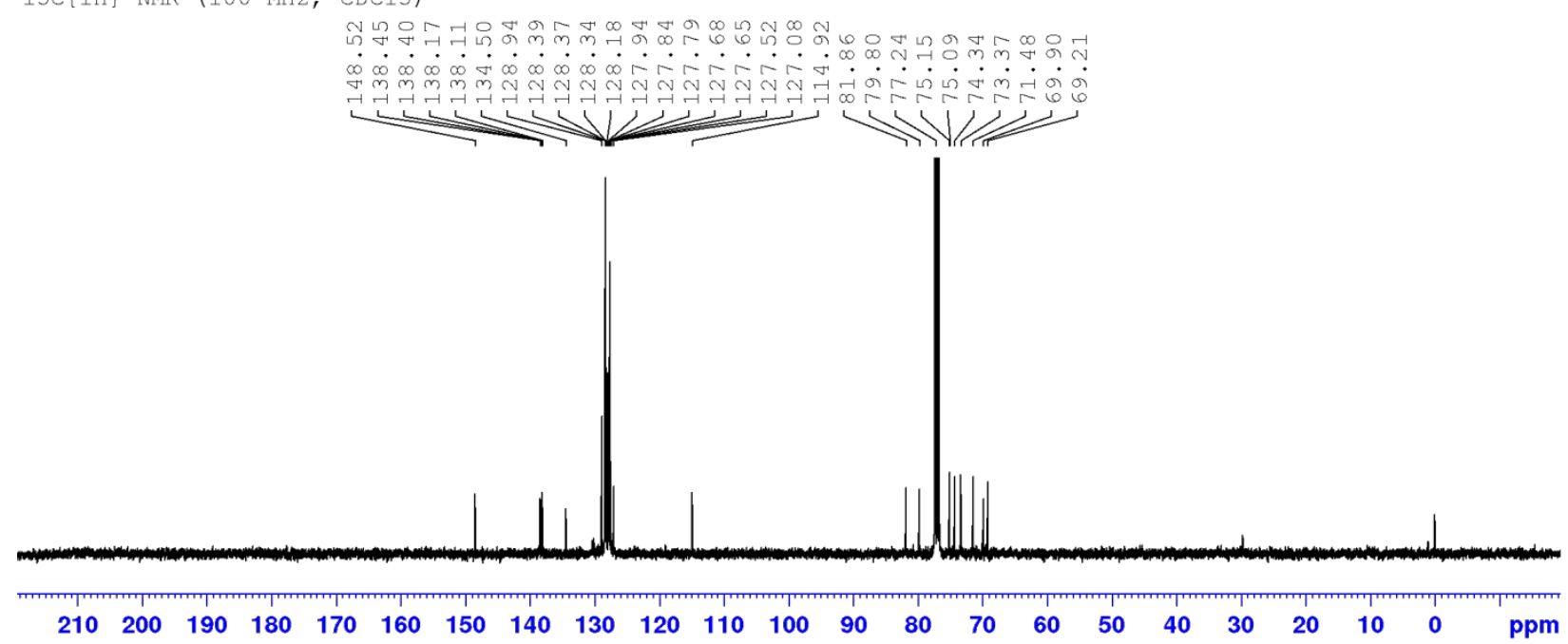
<smiles>OC[C@H]1O/C(=C\c2ccccc2)[C@H](O)[C@H]1OCc1ccccc1</smiles>

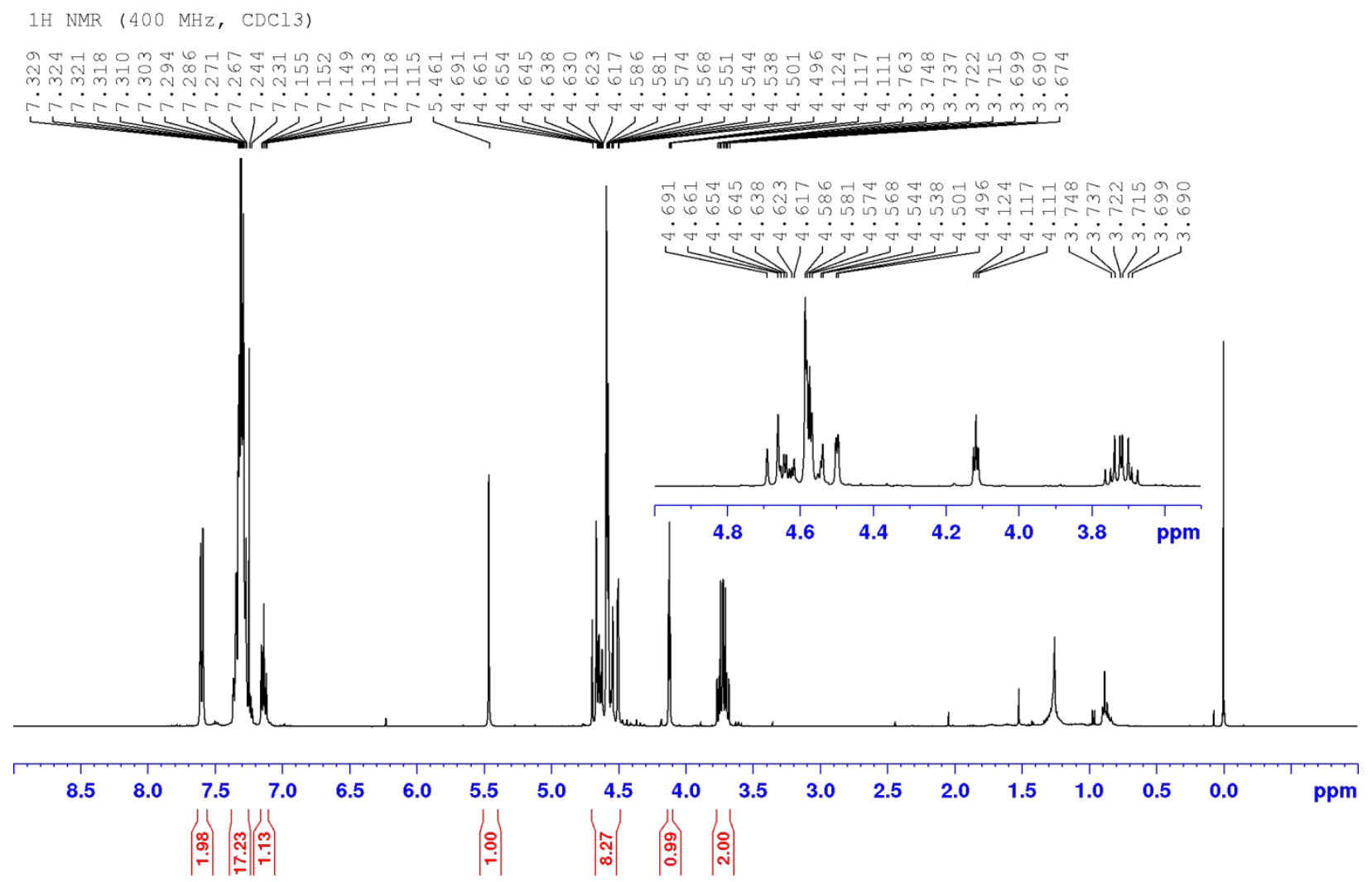

$13 \mathrm{C}\{1 \mathrm{H}\} \mathrm{NMR}(100 \mathrm{MHz}, \mathrm{CDCl} 3)$

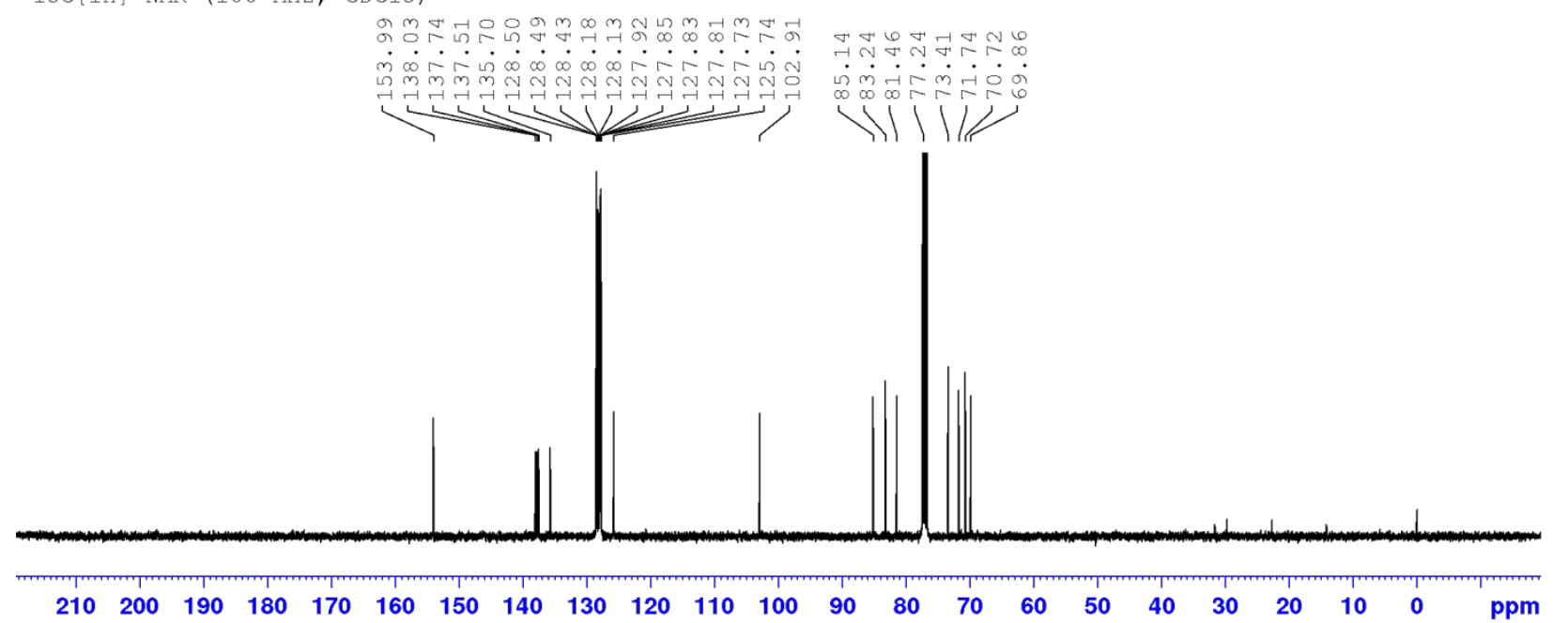


<smiles></smiles>

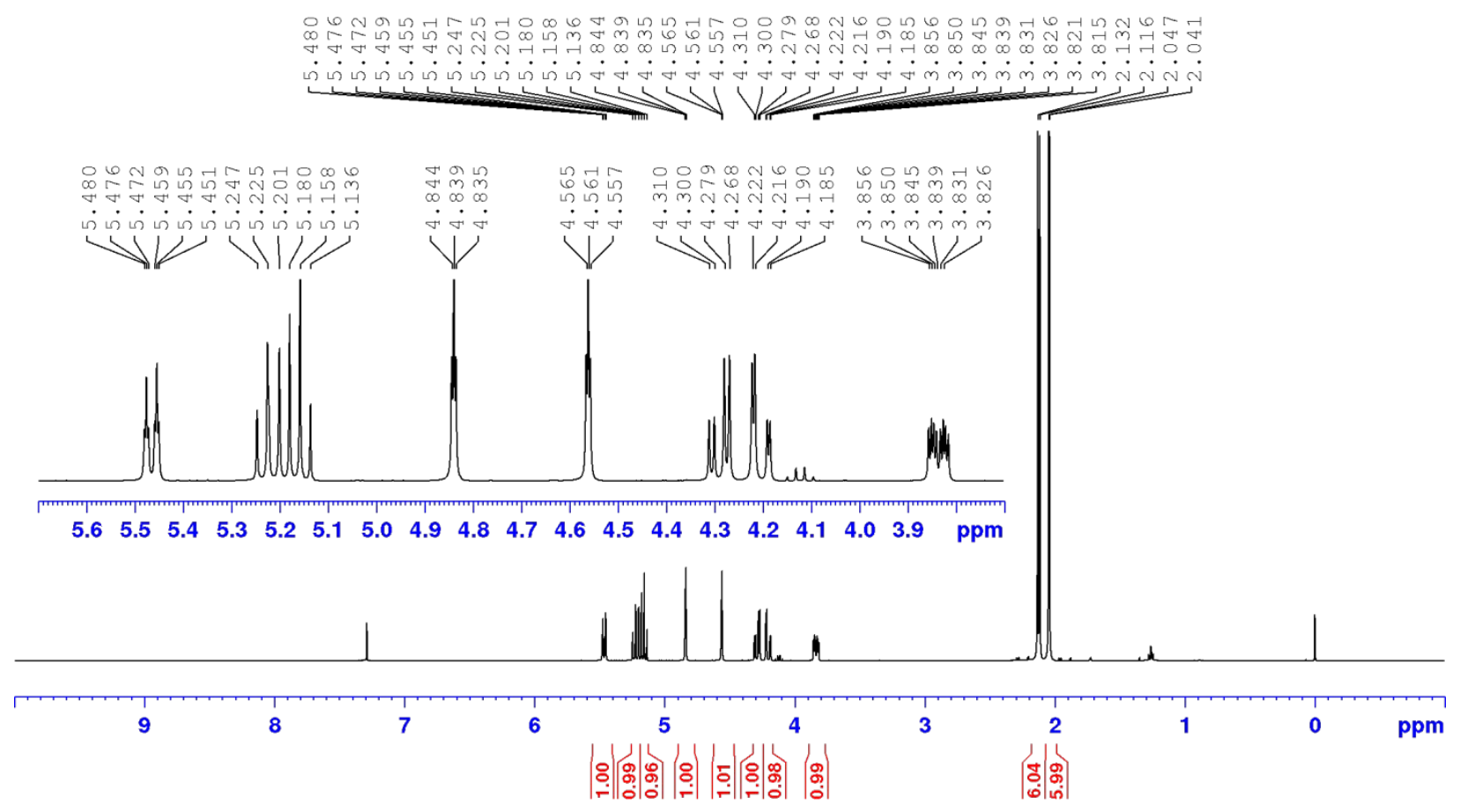

$13 \mathrm{C}\{1 \mathrm{H}\}$ NMR (100 $\mathrm{MHz}, \mathrm{CDCl} 3)$

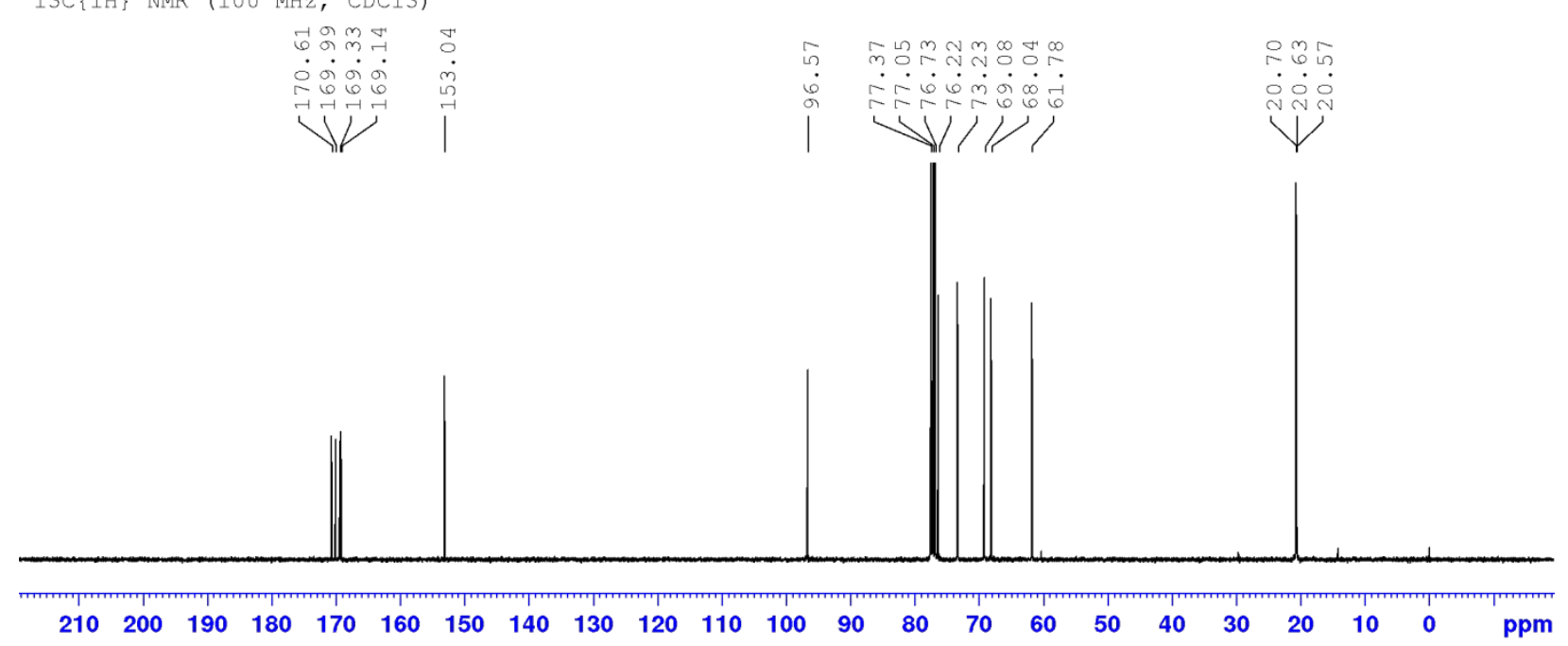


<smiles>CC(=O)OC[C@H]1O/C(=C\c2ccccc2)[C@H](OC(C)=O)[C@@H](OC(C)=O)[C@@H]1OC(C)=O</smiles>
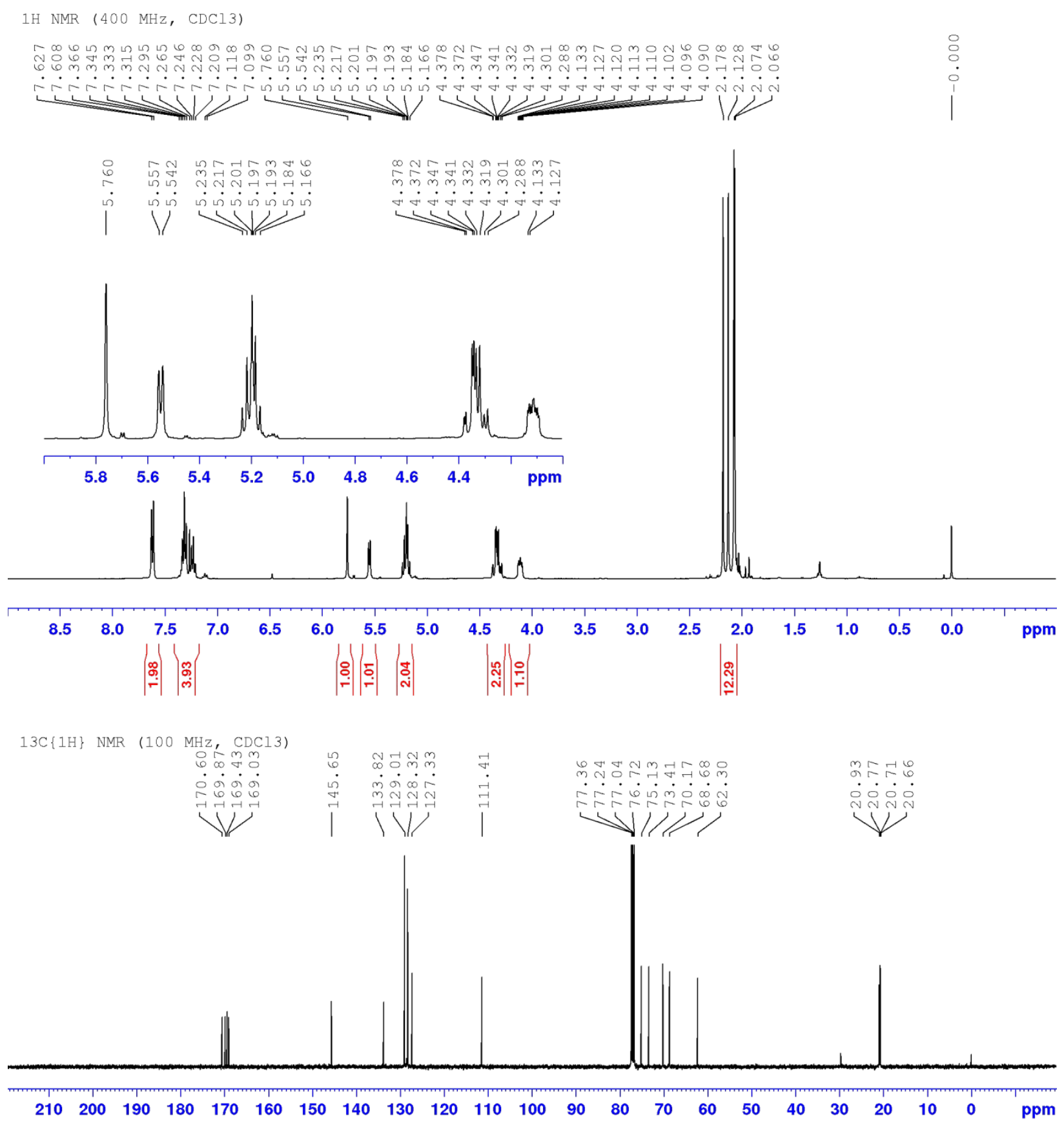


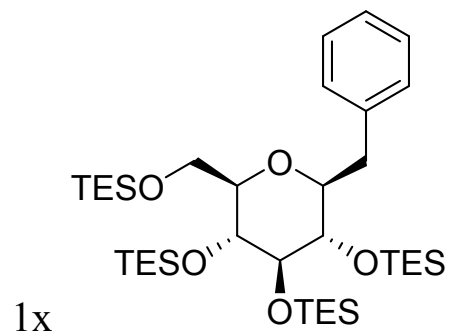

$1 \mathrm{H}$ NMR (400 MHz, CDCl3)

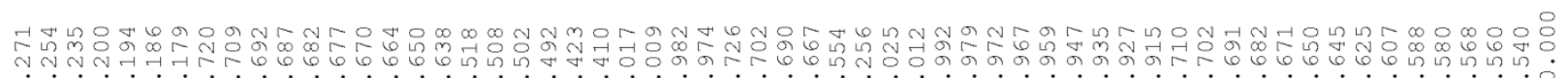

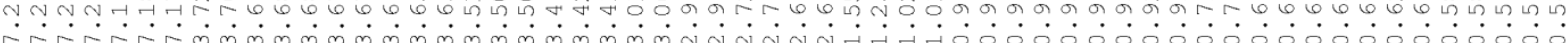
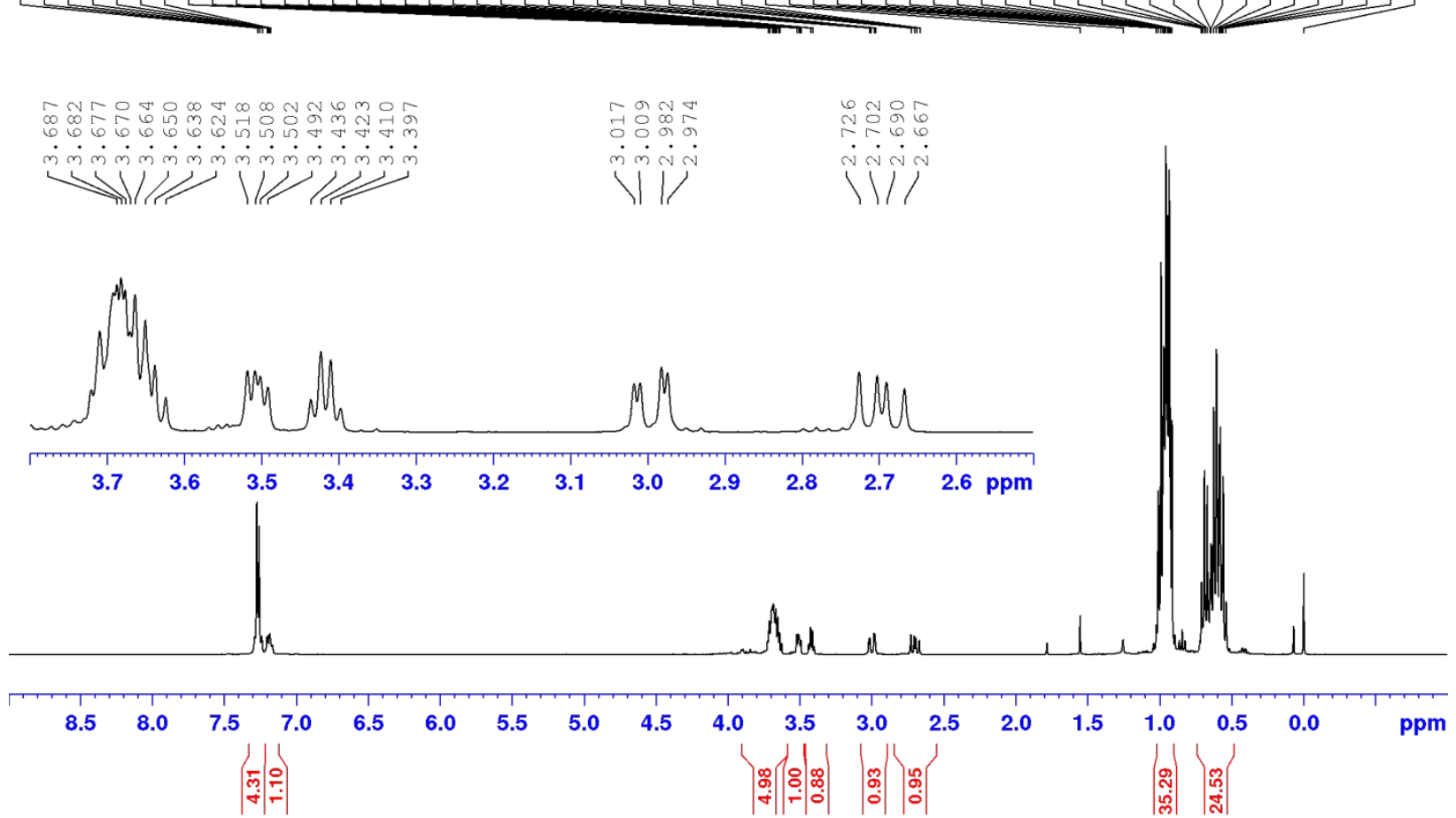

$13 \mathrm{C}\{1 \mathrm{H}\}$ NMR $(100 \mathrm{MHz}, \mathrm{CDCl} 3)$

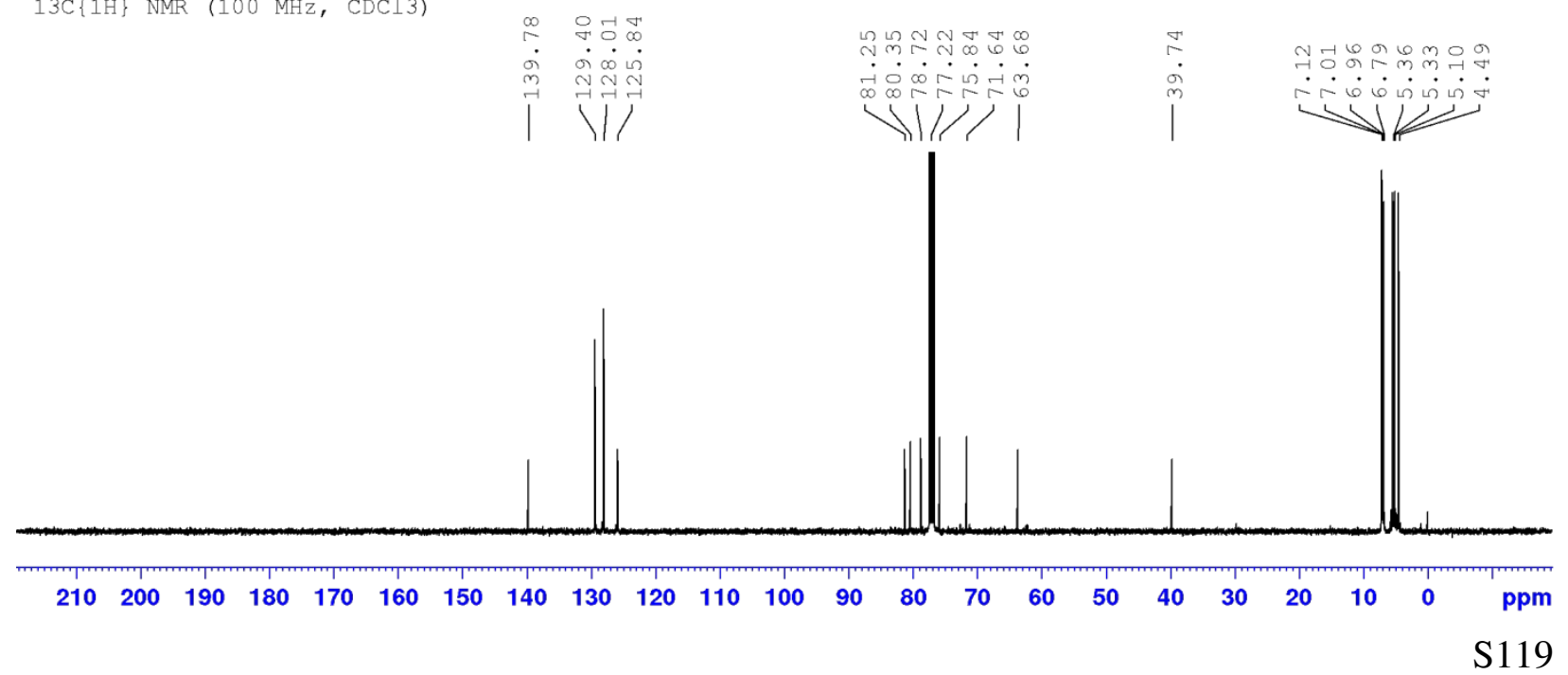


<smiles>CCOc1ccc(Cc2cc(I)ccc2Cl)cc1</smiles>
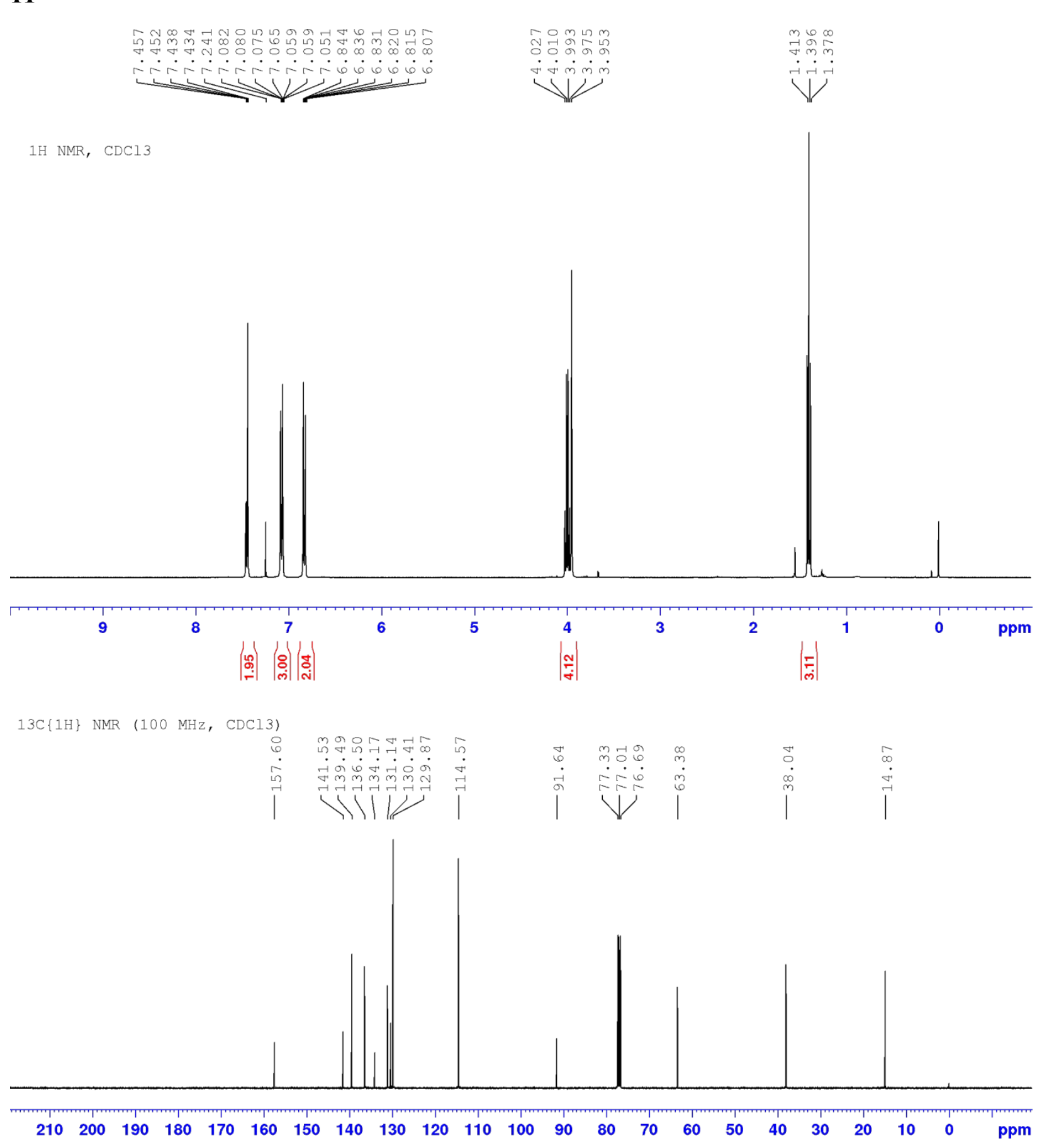


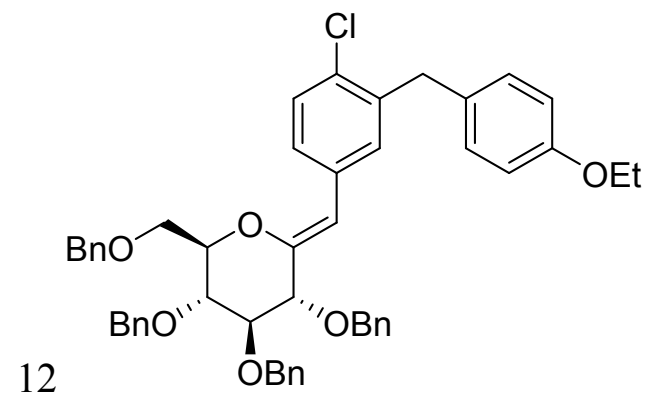

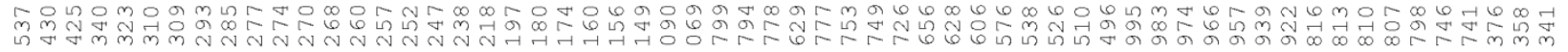

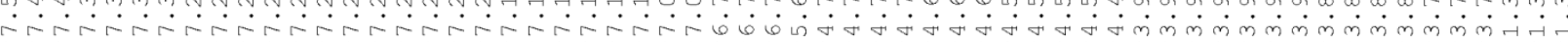

ता

$1 \mathrm{H}$ NMR, $\mathrm{CDCl} 3$

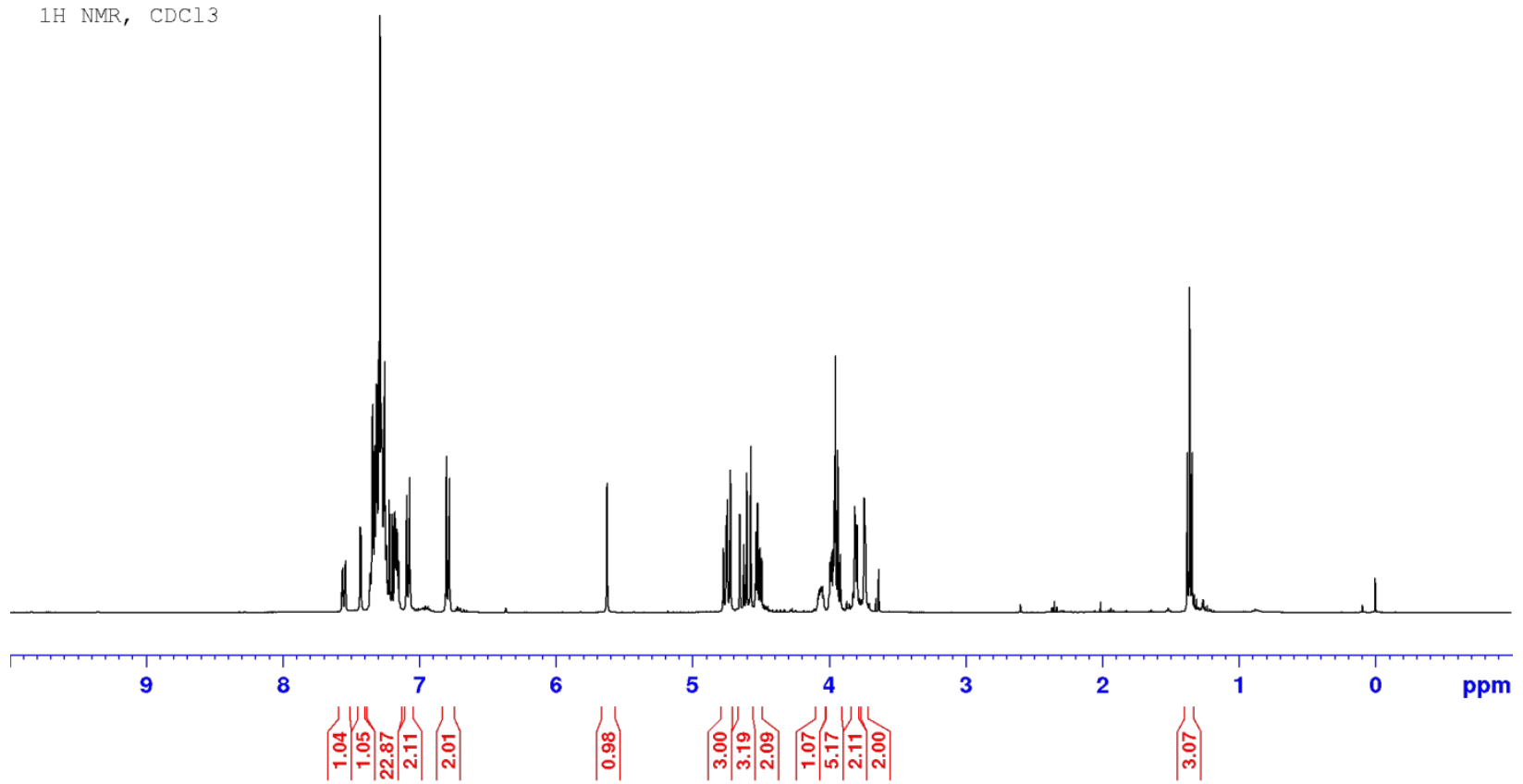




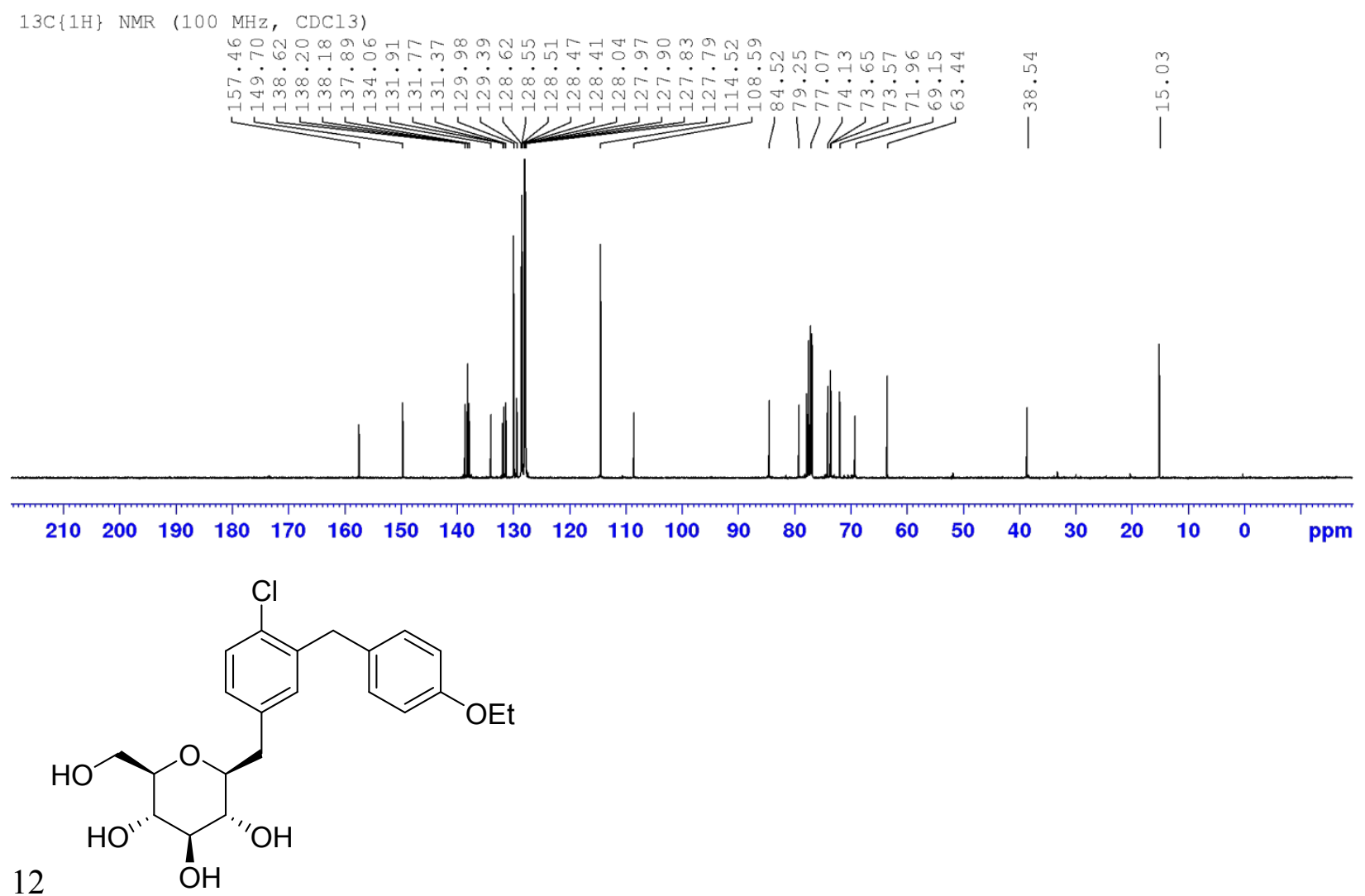



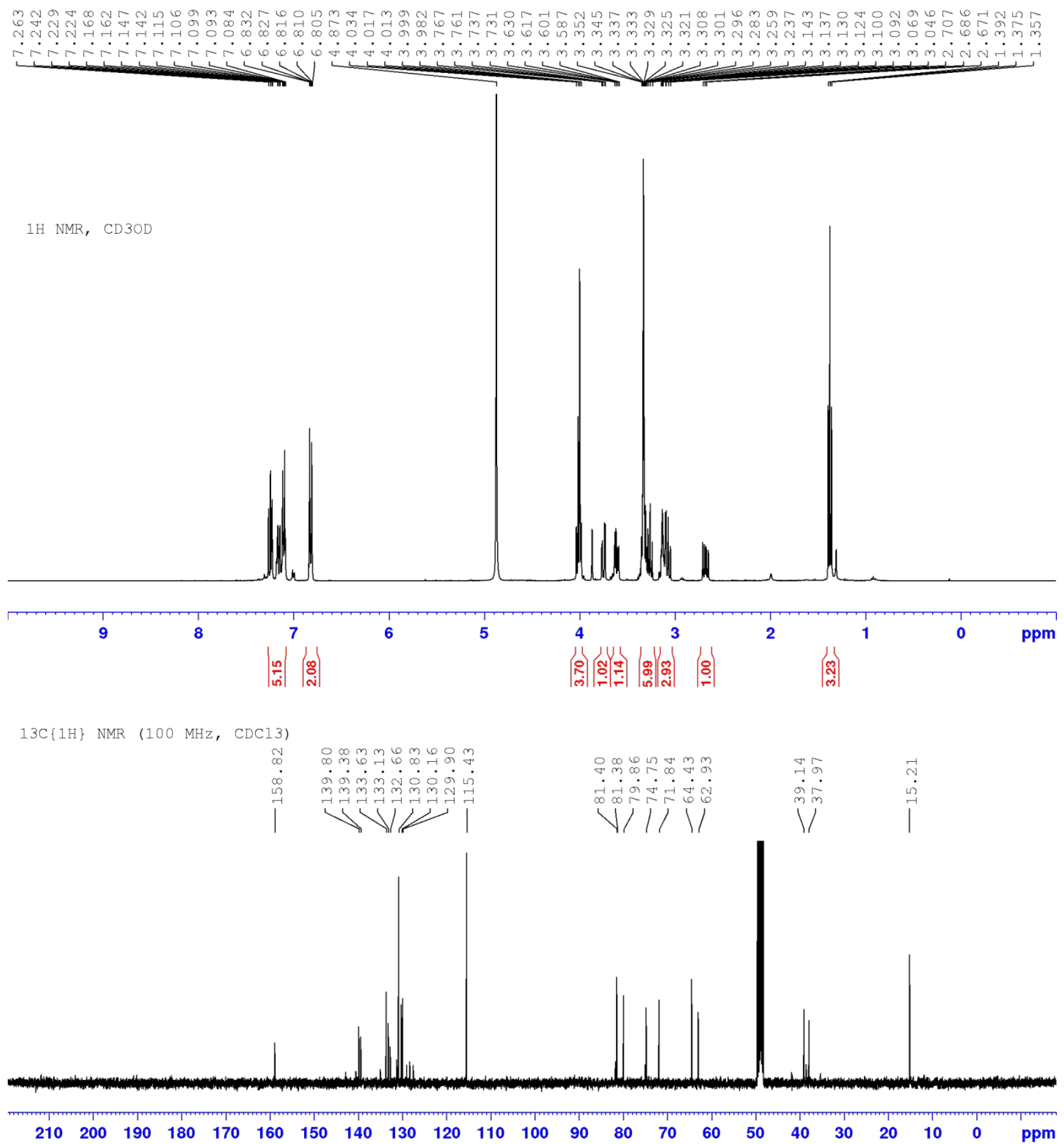